\title{
Emulsion microstructure and dynamics
}

\author{
By
}

Nelly Malassagne-Bulgarelli

\author{
A thesis \\ submitted to the Victoria University of Wellington \\ in fulfilment of the requirements for the degree of \\ Doctor of Philosophy \\ in Chemistry
}

Victoria University of Wellington 2010 


\section{ABSTRACT}

Emulsions are kinetically stabilised mixtures of two immiscible fluids (e.g. oil and water). They are encountered in many industrial applications including cosmetics, food, road, drug delivery and paint technology. Despite their wide spread use, the formulation of emulsions remains largely empirical. The nature of the relationships between ingredients, composition, emulsification method and energy input, defining the microstructure (e.g. droplet size distribution and surfactant packing at the oil/water interface), the dynamics (e.g. interdroplet exchange) and the lifetime of emulsions, is still poorly understood. In particular, little work has focused on the mutual interactions between emulsifier and oil molecules and how these affect the properties of the interfacial domain and emulsion dynamics.

The emulsion system oil/Triton X-100/water was investigated, where Triton X100 is a commercially available non ionic surfactant and the oil is one of toluene, $p$-xylene or octane. The microstructure and the dynamics of these oil/Triton $\mathrm{X}$ 100/water emulsions were monitored upon varying oil type, oil concentration, emulsion age and ionic strength while maintaining the oil-to-surfactant weight ratio, temperature, energy input and emulsification method constant. For this purpose, laser scanning confocal microscopy, cryo scanning electron microscopy (cryo-SEM), pulsed field gradient NMR (PFG-NMR), macroscopic phase separation and light scattering techniques were used as experimental techniques.

The occurrence of an oil exchange between oil droplets that is not coupled to droplet growth and emulsion destabilization is reported for the three oil systems: toluene, $p$-xylene or octane. The mixture of two separately stained emulsions, using green and red fluorescing dye molecules, leads to all droplets emitting yellow fluorescence under the confocal microscope within $~ 10$ min of mixing due to the interdroplet exchange of the two water insoluble dyes.

Furthermore, the PFG-NMR data for both toluene and $p$-xylene systems indicate that, for long observation times, $\Delta$, the echo attenuation of the oil signal decays as a single exponential upon increasing the diffusion parameters. In other words the individual motions of the droplets and oil molecules are described by a unique

diffusion coefficient belying the system polydispersity and indicative of a 
dynamic process occurring on a time scale faster than the observation time. One way to explain this outcome is to consider a motional averaging of the oil diffusion arising from either oil permeation upon droplet collision or reversible coalescence of the droplets. These two mechanisms are supported by the extensive droplet contact observed by cryo-SEM. Such an oil transfer occurring in three distinct oil systems, independently of emulsion destabilization, has not been reported previously.

Upon decreasing the NMR observation time below a specific value, $\Delta_{\text {switch, a }}$ switch of the echo attenuation data was detected between a single exponential and a multiexponential decay, the latter indicative of the emulsion droplet size distribution. The time scale of the oil transfer, $\Delta_{\text {switch, }}$ was probed upon varying oil type, oil concentration, emulsion age and ionic strength. In particular, the time scale of the oil exchange is an increasing function, spanning from $\sim 300 \mathrm{~ms}$ to $\sim 3$ $s$, of droplet concentration in toluene emulsions despite the concomitant increase of the droplet collision frequency. Upon increasing the toluene content and decreasing the mean interdroplet spacing, the oil droplets are kinetically stabilized by the enhancement of the surfactant packing at the oil/water interface.

In addition to the surfactant packing at the surface of the oil droplets, ionic strength and droplet size, the rate of oil exchange is controlled by the mutual interactions between oil and Triton X-100 molecules. The rate of oil transfer is a decreasing function from toluene to $p$-xylene to octane. The increase of the mean droplet size in the same order cannot solely account for the observed slowdown of the oil exchange. The macroscopic phase separation data indicate that the Triton $\mathrm{X}-100$ layer is increasingly robust with respect to oil transfer from toluene to $p$ xylene to octane. This can be compared with the oil exchange process and explained in terms of oil penetration effects into the surfactant layer and energy cost for hole nucleation. 


\section{ACKNOWLEDGEMENTS}

The first person to thank is my supervisor Kate McGrath. You have been a great supervisor over these past four years and I am very grateful. You have been very supportive and I really enjoyed working with you. Thanks to you, I have learnt a lot about emulsion science and scientific research in general. I know now that I would like to continue my career in this field.

A very special thank you to my wonderful husband Nicolas. You have been very supportive especially when I was working on weekends or when I was feeling a bit down after a failed experiment. You even put your professional plans on stand-by for me and for that I will always be very grateful.

Thanks to you Hui Lin for sharing with me the difficulties of being a PhD student and learning new experimental techniques (I am of course referring to diffusion NMR) and above all for being a very nice colleague. I hope we will get the chance to work together in the future, who knows?

Many thanks to Antoine Lutti, Mark Hunter and Kirk Feindel for helping me out with the NMR. You took the time to sit down and help me out with the pulse sequence and automation program. Without you guys it would have been much more difficult for me.

I also thank all my group members including Sheinach Dunn, Hui Lin Tan, Benjamin Matthewson, Conrad Lendrum, Ben Murray, Agathe Fournier, Catherine Davis, Nguyen Nguyen, Nick Monahan, Ash Dangerfield, Hemi Cumming and David Green. It was nice working in the same group and having group activities with you guys.

Finally I thank the MacDiarmid Institute for Advanced Materials and Nanotechnology for funding my $\mathrm{PhD}$ studies and giving me the opportunity to work in this very welcoming country that New Zealand is. 


\section{TABLE OF CONTENTS}

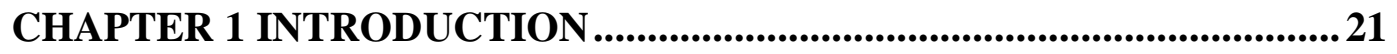

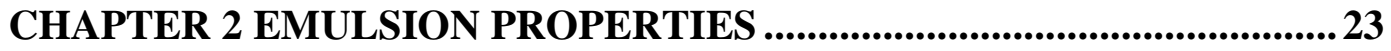

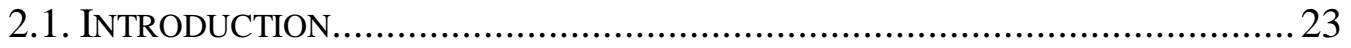

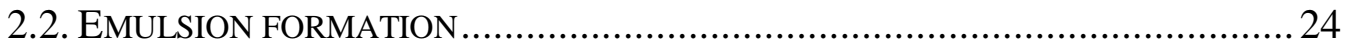

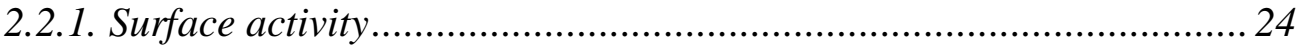

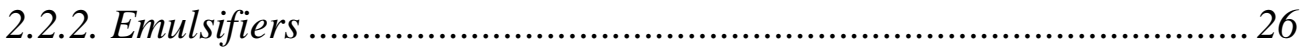

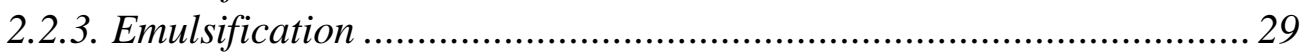

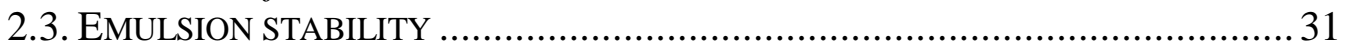

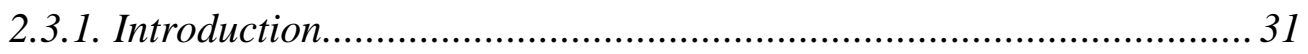

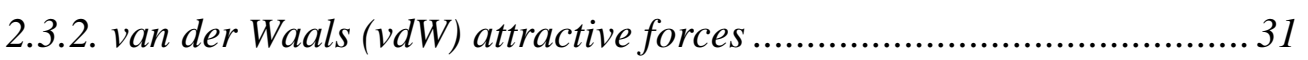

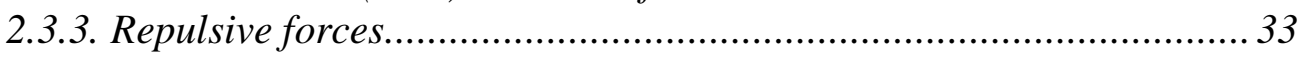

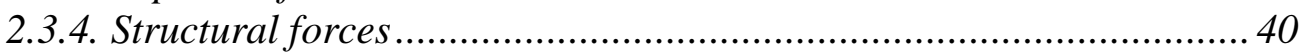

2.4. MECHANISMS OF EMULSION DESTABILIZATION ........................................ 41

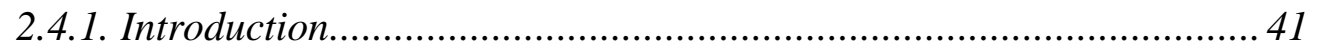

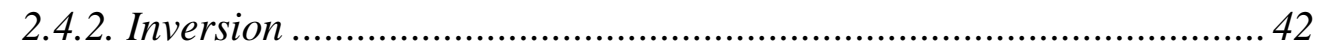

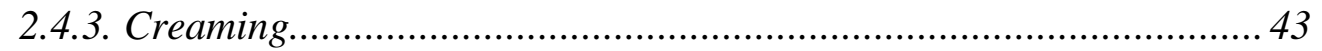

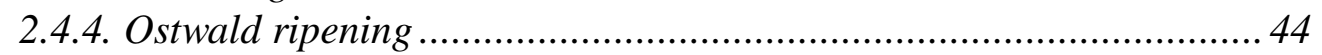

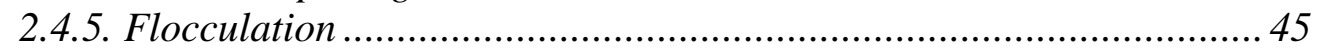

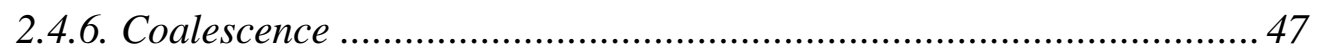

CHAPTER 3 TOLUENE/TRITON X-100/WATER EMULSIONS ............... 51

3.1. CHOICE OF THE EMULSIFIER ............................................................... 51

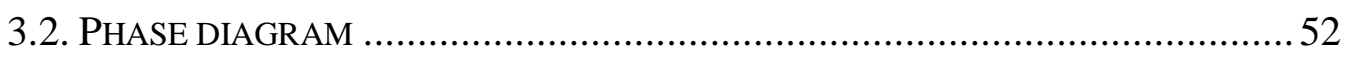

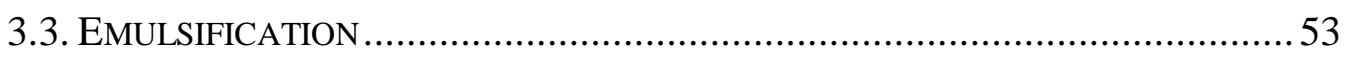

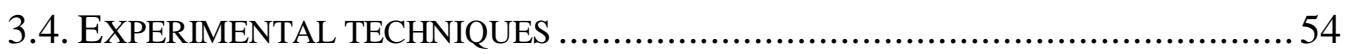

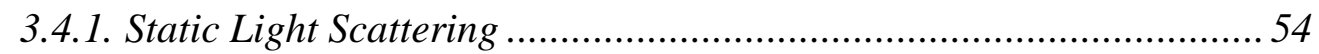

3.4.2. Dynamic Light Scattering ............................................................ 58

3.4.3. Pulsed field gradient nuclear magnetic resonance ............................ 62

3.4.4. Laser Scanning Confocal Microscopy ............................................ 73

3.4.5. Cryo Scanning Electron Microscopy................................................ 76

3.4.6. Rate of macroscopic phase separation ............................................. 80

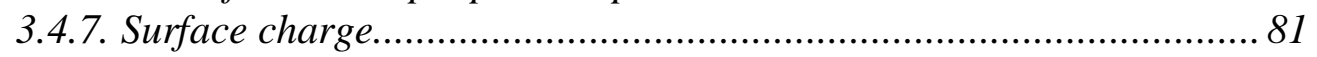

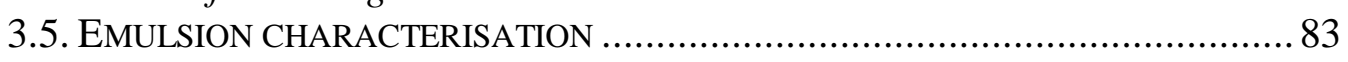

CHAPTER 4 OIL EXCHANGE IN TOLUENE/TRITON X-100/WATER

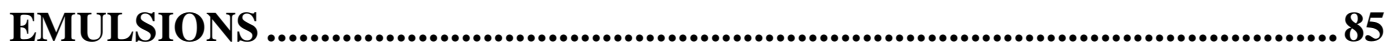

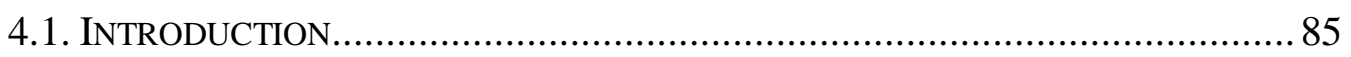

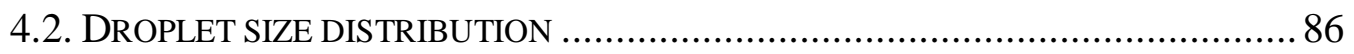

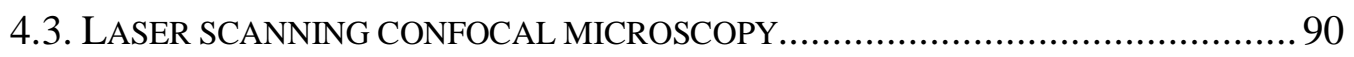

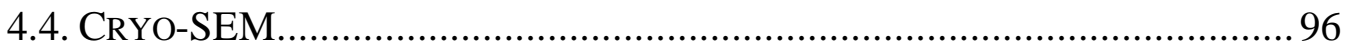

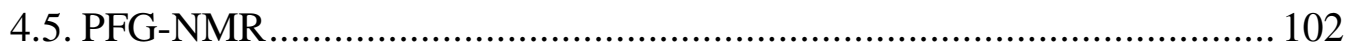

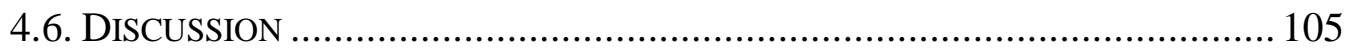

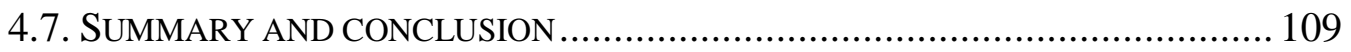

CHAPTER 5 KINETICS OF OIL EXCHANGE ........................................111

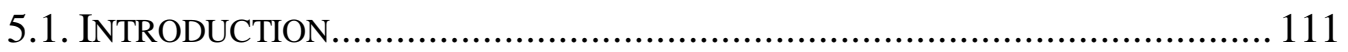




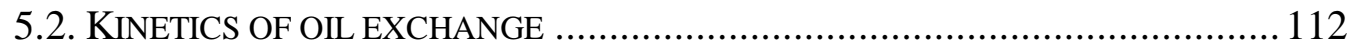

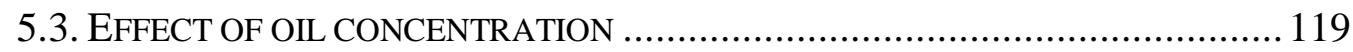

5.4. ADDITION OF BACKGROUND ELECTROLYTE .......................................... 130

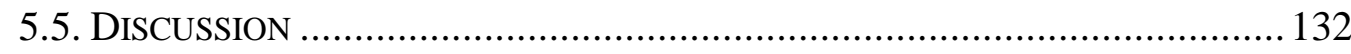

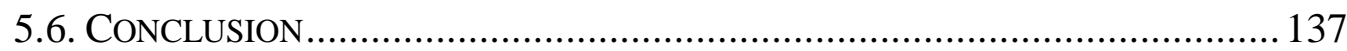

CHAPTER 6 AGEING OF TOLUENE/TRITON X-100/WATER

EMULSIONS .......................................................................................... 139

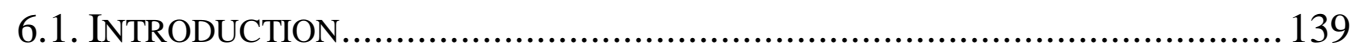

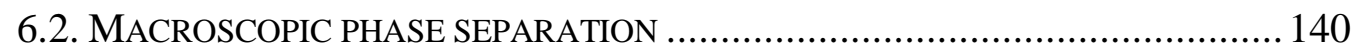

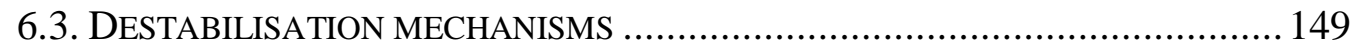

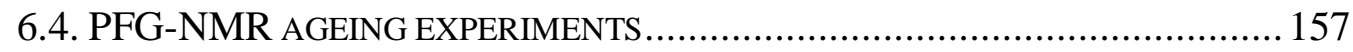

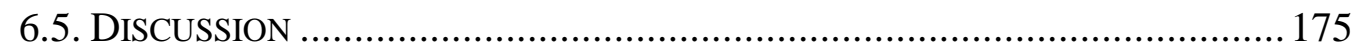

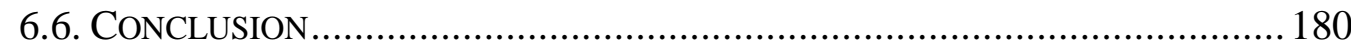

CHAPTER 7 VARIATION OF THE NATURE OF THE OIL IN OIL/TRITON X-100/WATER EMULSIONS............................................. 183

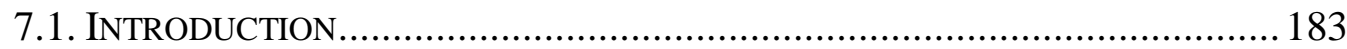

7.2. THE MICROSTRUCTURE OF P-XYLENE/TRITON X-100/WATER EMULSIONS. 184

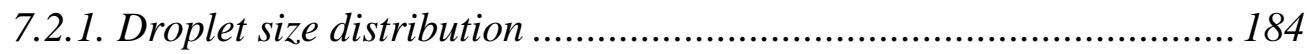

7.2.2. Macroscopic phase separation....................................................... 187

7.3. EFFECT OF THE OIL ON THE DYNAMICS OF OIL TRANSFER......................... 194

7.3.1. Laser scanning confocal microscopy.......................................... 194

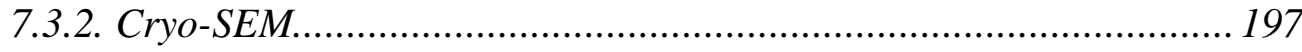

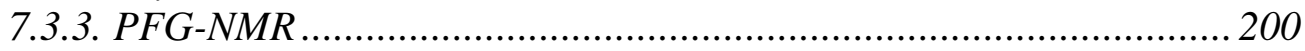

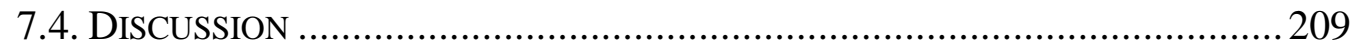

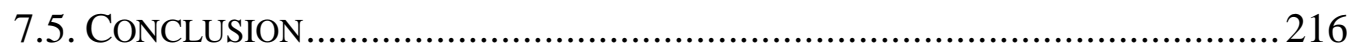

CHAPTER 8 GENERAL CONCLUSION AND FUTURE WORK ............ 217

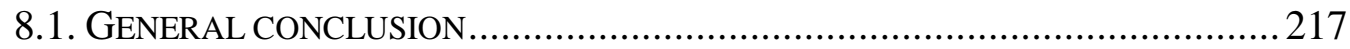

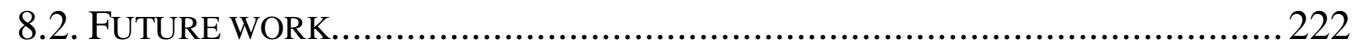




\section{TABLE OF FIGURES}

FIGURE 1.1. FORMATION OF AN OIL-IN-WATER (O/W) EMULSION. ......................... 21

FIGURE 2.1. DROPLET DIMENSION FOR LIQUID-LIQUID DISPERSED SYSTEMS. ......... 23

FIGURE 2.2. VAN DER WAALS FORCES IN A BULK LIQUID. IN THE ABSENCE OF AN INTERFACE, THE NET ATTRACTIVE FORCE F FELT BY A MOLECULE IS EQUAL TO ZERO WHEREAS IN THE PRESENCE OF AN INTERFACE, THE NET ATTRACTIVE FORCE IS POSITIVE.

FIGURE 2.3. LOCATION OF THE EMULSIFIER AT THE OIL/WATER INTERFACE IN AN O/W EMULSION (NOT TO SCALE) ............................................................. 26

FIGURE 2.4. SHEAR UNDERGONE BY A DROPLET DURING THE EMULSIFICATION PROCESS.

FIGURE 2.5. EMULSIFICATION TECHNIQUES (A) ROTOR-STATOR DISPERSER, (B) HIGH PRESSURE HOMOGENIZER AND (C) JET IMPACT DEVICE................................ 30

FIGURE 2.6. INTERACTION ENERGY-DISTANCE PLOT FOR TWO DROPLETS APPROACHING EACH OTHER IN THE ABSENCE OF EMULSIFIER, $A_{H}=3.7 \times 10^{-21} \mathrm{~J}$; $A=0.133 \mu \mathrm{M}$. 33

FIGURE 2.7. STRUCTURE OF THE ELECTRICAL DOUBLE LAYER AT THE OIL/WATER INTERFACE WITH NEGATIVELY CHARGED ADSORBED EMULSIFIER.... 35

FIGURE 2.8. INTERACTION ENERGY FOR TWO COLLOIDAL PARTICLES AS A FUNCTION OF THEIR DISTANCE OF SEPARATION, FOR ELECTRICAL DOUBLE LAYERS DUE TO SURFACE CHARGE (SQUARES, $G_{R}$ ), LONDON-VAN DER WAALS DISPERSION FORCES (TRIANGLES, $G_{A}$ ), AND THE TOTAL INTERACTION (BOLD LINE, $G_{D L V O}$ ) CALCULATED WITH $A=3.7 \times 10^{-21} \mathrm{~J} ; A=0.133 \mu \mathrm{M} ; \varepsilon_{R}=78.54 \mathrm{~F} / \mathrm{M}$ AND $\psi_{O}=$ $0.01 \mathrm{~V}$.

FIGURE 2.9. TOTAL INTERACTION ENERGY FOR TWO COLLOIDAL PARTICLES AS A FUNCTION OF THEIR DISTANCE OF SEPARATION DUE TO STERIC REPULSION AND VDW ATTRACTION.

FIGURE 2.10. INVERSION OF AN O/W IN A W/O EMULSION. .............................. 42

FIGURE 2.11. CREAMING OF AN OIL-IN-WATER EMULSION. ................................ 43

FIGURE 2.12. OSTWALD RIPENING IN AN O/W EMULSION. ................................ 44

FIGURE 2.13. FLOCCULATION IN AN O/W EMULSION........................................ 46

FIGURE 2.14. DEPLETION FLOCCULATION IN AN O/W EMULSION. ........................ 47

FIGURE 2.15. COALESCENCE IN AN O/W EMULSION. ........................................... 47

FIGURE 2.16. LAMELLA FORMATION BETWEEN DROPLETS. .................................. 48

FigURE 3.1. CHEMICAL FORMULA OF TRITON X-100; $N=10$ ON AVERAGE............. 51

FIGURE 3.2. PHASE DIAGRAM OF THE TOLUENE/TRITON X-100/WATER SYSTEM TAKEN FROM REFERENCE [40]. MICROEMULSIONS ARE FORMED IN THE HASHED REGION, LIQUID CRYSTALS IN THE STRIPES, TWO-PHASE WINSOR EMULSIONS IN THE SOLID ZONE, AND EXTREMELY TEMPERATURE SENSITIVE TWO- AND THREEPHASE WINSOR EMULSIONS IN THE BRICKED AREA. THE STRAIGHT LINE INDICATES THE REGION WHERE STABLE EMULSIONS ARE FORMED WITH A CONSTANT RATIO OF 1:5 TRITON X-100/TOLUENE....................................... 52

FIGURE 3.3. BLENDER FROM WARRING USED FOR EMULSIFICATION..................... 53

FIGURE 3.4. MASTERSIZER 2000 FROM MALVERN INSTRUMENTS. ......................... 56

FIGURE 3.5. PRINCIPLE OF PARTICLE SIZING BY SLS; THE LIGHT SOURCE EMITTED BY A LASER IS SCATTERED BY THE PARTICLES WITH AN INTENSITY AND AT AN ANGLE WHICH ARE SIZE DEPENDENT. THE FOCAL PLANE DETECTOR IS 
SENSITIVE TO SCATTERING BY LARGE PARTICLES WHEREAS WIDE ANGLE DETECTORS ARE SENSITIVE TO THE LIGHT SCATTERED BY SMALL PARTICLES. 56

FIGURE 3.6. DROPLET SIZE DISTRIBUTION OF A TOLUENE/TRITON X-100/WATER EMULSION PREPARED WITH 20 WT\% TOLUENE.

FIGURE 3.7. A TYPICAL DLS INSTRUMENT; THE LIGHT EMITTED BY A LASER SOURCE IS SCATTERED BY PARTICLES AND DETECTED AT WIDE ANGLES BY A

BACKSCATTERED DETECTOR AT SUCCESSIVE TIME INTERVALS.

FIGURE 3.8. CORRELATION FUNCTION FOR DIFFERENT PARTICLE SIZES. ................61

FIGURE 3.9. SIZE DISTRIBUTION OF THE OIL SWOLLEN MICELLES PRESENT IN A TOLUENE/TRITON X-100/ WATER EMULSION PREPARED WITH 15 WT\% TOLUENE

FIGURE 3.10. PGSE PULSE SEQUENCE (A); POSITION OF THE SPINS DURING THE PGSE IN THE ABSENCE (B) OR PRESENCE (C) OF DIFFUSION DURING THE OBSERVATION TIME, $\triangle$.

FiguRE 3.11. PULSED GRADIENT STIMULATED ECHO PULSE SEQUENCE. THREE RADIO FREQUENCY PULSES ROTATE THE NET MAGNETIZATION TO A $\pi / 2$ ANGLE. A FIRST GRADIENT OF AMPLITUDE $G$ AND DURATION $\delta$ LABELS THE POSITIONS OF THE SPINS WHEREAS A SECOND GRADIENT, AFTER AN OBSERVATION TIME, $\Delta$, RECOVERS THE SIGNAL TO FORM AN ECHO..................................................66

FigURE 3.12. BRUKER AVANCE-460 SPECTROMETER. .......................................6 67

FIGURE 3.13. SCHEMATIC OF THE KEY COMPONENTS OF A SPECTROMETER. A SUPERCONDUCTING SOLENOID PRODUCES AN INTENSE MAGNETIC FIELD AND SHIM COILS OPTIMISE THE FIELD HOMOGENEITY. THE SAMPLE IS INSERTED INTO A PROBE WHICH PRODUCES $\pi / 2$ RADIO FREQUENCY PULSES (EXCITATION) AND DETECT THE FREE INDUCTION DECAY (SIGNAL).

FIGURE 3.14. ECHO ATTENUATION FOR PURE MILLIQ WATER, FOR AN OBSERVATION TIME, $\Delta$, OF $20 \mathrm{MS}, \mathrm{T}=298 \mathrm{~K}$.

FIGURE 3.15. ECHO ATTENUATION OF THE OIL SIGNAL IN A TOLUENE/TRITON X100/WATER EMULSION PREPARED WITH 40 WT\% TOLUENE AND 8 WT\% TRITON $\mathrm{X}-100, \Delta=40 \mathrm{MS}$. .72

FIGURE 3.16. DIFFUSION SPECTRUM OF THE OIL SIGNAL IN A TOLUENE/TRITON X100/WATER EMULSION PREPARED WITH 40 WT\% TOLUENE AND 8 WT\% TRITON $\mathrm{X}-100, \Delta=40 \mathrm{MS}$

FIGURE 3.17. COMPARISON OF WIDE FIELD (TOP PICTURES) AND CONFOCAL (BOTTOM PICTURES) IMAGING, (A) AND (D) HUMAN MEDULLA, (B) AND (E) RABBIT MUSCLES FIBRES, (C) AND (F) SUNFLOWER POLLEN GRAIN TAKEN FROM REFERENCE [65].

FIGURE 3.18. PRINCIPLE OF CONFOCAL MICROSCOPY. LIGHT IS EMITTED BY A LASER AND IS SCANNED ACROSS THE SAMPLE BY ROTATING MIRRORS. FLUORESCENT LIGHT EMITTED OFF THE SAMPLE THAT IS NOT FROM THE OBJECTIVE LENS'S FOCAL PLANE IS REJECTED BY A SCREEN AND A PINHOLE.

FIGURE 3.19. 2D CONFOCAL SNAPSHOTS $256 \times 256(\times 100$ OBJECTIVE) OF WATER/TRITON X-100/TOLUENE EMULSIONS PREPARED WITH 15 WT\% TOLUENE AND 3 WT\% TRITON X-100. (A) EMULSION LABELLED WITH A RED FLUOROPHORE. (B) EMULSION LABELLED WITH A GREEN FLUOROPHORE. (C) MIXTURE OF BOTH EMULSIONS (A) AND (B).

FIGURE 3.20. CRYO SCANNING ELECTRON MICROSCOPE FROM JEOL GATAN.......... 78

FigURE 3.21. PRINCIPLE OF SEM. AN ELECTRON BEAM, GENERATED BY AN ELECTRON GUN, IS ACCELERATED BY AN ANODE, FOCUSED BY A SERIES OF 
LENSES AND SCANNED ACROSS THE SAMPLE USING SCANNING COILS. THE ELECTRON BEAM IS SCATTERED BY THE SAMPLE ATOMS AND DETECTED IN BACKSCATTERED OR SECONDARY ELECTRON MODE.

FIGURE 3.22. VOLUME OF INTERACTION BETWEEN THE ELECTRON BEAM AND THE SAMPLE. SECONDARY ELECTRONS ARE GENERATED BY SCATTERING OF THE OUTER-SHELL ELECTRONS. BACKSCATTERED ELECTRONS ARE DIRECTLY SCATTERED BY THE ATOMIC NUCLEI WHEREAS CHARACTERISTIC X-RAYS RESULT FROM THE SCATTERING OF THE INCOMING ELECTRONS BY INNER-SHELL ELECTRONS UPON RELAXATION

FIGURE 3.23. EVALUATION OF THE RATE OF MACROSCOPIC PHASE SEPARATION OF A TOLUENE/TRITON X-100/WATER EMULSION, HERE 15 WT\% OIL. 80

FIGURE 3.24. RATE OF MACROSCOPIC PHASE SEPARATION FOR A TOLUENE/TRITON X-100/WATER EMULSION PREPARED WITH 15 WT\% (TRIANGLES) AND 35 WT\% TOLUENE (SQUARES).

FIGURE 3.25. FOLDED CAPILLARY CELL USED IN AN ELECTROPHORESIS EXPERIMENT.

FIGURE 4.1. DROPLET SIZE DISTRIBUTIONS OF EMULSIONS BETWEEN 5 AND 70 WT\% TOLUENE GENERATED BY SLS. 86

FIGURE 4.2. NUMBER, INTENSITY AND VOLUME DROPLET SIZE DISTRIBUTIONS MEASURED BY DLS AND VOLUME DROPLET SIZE DISTRIBUTION MEASURED BY SLS FOR A FRESH EMULSION PREPARED WITH 5 WT\% TOLUENE.

FIGURE 4.3. TEM MICROGRAPH OF A CLOSED-CELL FOAM COEXISTING WITH A DISPERSED DROPLET O/W EMULSION WITH 65 WT\% TOLUENE TAKEN FROM REFERENCE [40].

FIGURE 4.4. DROPLET SIZE DISTRIBUTIONS OF THE EXPELLED AQUEOUS PHASE OF AGED TOLUENE/TRITON X-100/WATER EMULSIONS BETWEEN 5 AND 60 WT\% TOLUENE USING DLS.

FIGURE 4.5. DROPLET SIZE DISTRIBUTIONS OF DILUTED FRESH TOLUENE/TRITON X100/WATER EMULSIONS PREPARED WITH 5 WT\% TOLUENE USING DLS. ........ 90

FigURE 4.6. CHEMICAL FORMULA OF NILE RED (A), BODIPY 665/676 (B), 4-(4METHOXYBENZYLAMINO)-7-NITROBENZOFURAZAN (C) AND BODIPY 493/503 (D)......

FIGURE 4.7. 2D CONFOCAL MICROSCOPY OPTICAL CROSS-SECTION 256×256 (×100 OBJECTIVE, ZOOM FACTOR 4) OF A 15 WT\% TOLUENE EMULSION SAMPLE LABELLED WITH NILE RED. SNAPSHOT TAKEN 30 MINUTES AFTER EMULSIFICATION.

FIGURE 4.8. DROPLET SIZE DISTRIBUTION OF A 15 WT\% TOLUENE EMULSION SAMPLE LABELLED WITH NILE RED (STRAIGHT LINE) AND WITH NO DYE ADDITION (SQUARES) OBTAINED BY SLS.

FIGURE 4.9. LINK TO OPTICAL CROSS-SECTION COLLECTED OVER TIME AT 428 MS/FRAME, 256×256 PIXELS, ZOOM FACTOR 4 FOR A FRESHLY EMULSIFIED 15 WT\% TOLUENE EMULSION SAMPLE LABELLED WITH NILE RED USING CONFOCAL MICROSCOPY.

FIGURE 4.10. 2D CONFOCAL MICROSCOPY OPTICAL CROSS-SECTION 256×256 $(\times 100$ OBJECTIVE, ZOOM FACTOR 4) OF A 15 WT\% TOLUENE EMULSION SAMPLE LABELLED WITH 4-(4-METHOXYBENZYLAMINO)-7-NITROBENZOFURAZAN. SNAPSHOT TAKEN 35 MINUTES AFTER EMULSIFICATION.....

FIGURE 4.11. 2D CONFOCAL MICROSCOPY OPTICAL CROSS-SECTION 256×256 ( $\times 100$ OBJECTIVE, ZOOM FACTOR 4) OF A 15 WT\% TOLUENE EMULSION SAMPLE PREPARED BY MIXING A RED FLUORESCING AND A GREEN FLUORESCING 
EMULSION SAMPLE. SNAPSHOT TAKEN WITHIN 10 MINUTES OF MIXING AND 40 MINUTES AFTER EMULSIFICATION.

FIGURE 4.12. POSTULATED MECHANISM FOR THE REVERSIBLE COALESCENCE OF DROPLETS.

FIGURE 4.13. CRYO-SEM IMAGE OF A FRESHLY EMULSIFIED 40 WT\% TOLUENE EMULSION SAMPLE $(\times 1000)$.

FIGURE 4.14. CRYO-SEM IMAGE OF A FRESHLY EMULSIFIED 40 WT\% TOLUENE EMULSION SAMPLE $(\times 5000)$.

FIGURE 4.15. CRYO-SEM IMAGE OF A FRESHLY EMULSIFIED 15 WT\% TOLUENE EMULSION SAMPLE $(\times 5,000)$.

FIGURE 4.16. CRYO-SEM IMAGE OF A FRESHLY EMULSIFIED 15 WT\% TOLUENE EMULSION SAMPLE $(\times 20,000)$.

FIGURE 4.17. CRYO-SEM IMAGE OF A MELTED 40 WT\% TOLUENE EMULSION $(\times 2,000)$.

FIGURE 4.18. CRYO-SEM IMAGE OF A MELTED 40 WT\% TOLUENE EMULSION $(\times 5,000)$. 101

FIGURE 4.19. REPRESENTATIVE DIFFUSION ECHO ATTENUATION OF THE OIL SIGNAL OF EMULSIONS FRESHLY PREPARED BETWEEN 5 AND 55 WT\% TOLUENE, HERE 40 WT\%. THE OBSERVATION TIME, $\Delta$, SPANS FROM 300 MS TO 3 S DEPENDING ON OIL CONCENTRATION. 103

FIGURE 4.20. REPRESENTATIVE DIFFUSION ECHO ATTENUATION OF THE OIL SIGNAL OF EMULSIONS FRESHLY PREPARED BETWEEN 5 AND 55 WT\% TOLUENE, HERE 15 WT\%, BY REPLACING WATER WITH $\mathrm{D}_{2} \mathrm{O}$.

FIGURE 5.1. DIFFUSION ECHO ATTENUATION OF THE OIL SIGNAL FOR A FRESH 40 WT\% TOLUENE EMULSION. A SINGLE EXPONENTIAL DECAY IS OBSERVED FOR AN OBSERVATION TIME, $\Delta$, BETWEEN $1.3 \mathrm{~s}\left(\Delta_{\text {SWITCH }}\right)$ AND $3 \mathrm{~S}$ WHEREAS A MULTIEXPONENTTIAL DECAY IS MEASURED FOR $\triangle$ BETWEEN 6.3 MS AND $1.2 \mathrm{~s}$. THESE ECHO ATTENUATIONS ARE REPRESENTATIVE OF EMULSION SAMPLES BETWEEN 5 AND 55 WT\% TOLUENE.

FIGURE 5.2. REPRESENTATIVE DIFFUSION SPECTRA OF TOLUENE MOLECULES AND DROPLETS, DETERMINED AT VARIOUS EXPERIMENTAL OBSERVATION TIMES ( $\Delta$ $=20,30,40,50$ AND $60 \mathrm{MS}$, ALL BELOW $\Delta_{\text {SWITCH }}=1.3 \mathrm{~s}$ ) FOR EMULSIONS PREPARED WITH 40 WT\% TOLUENE. AT SHORT $\Delta$ VALUES, THREE PEAKS NUMBERED 1,2 AND 3 MAY BE DISTINGUISHED.

FiguRE 5.3. PRODUCT $D \Delta$ VERSUS $\Delta$ FOR THE LOW END TAIL, $D_{\text {LOW }}$ MAXIMUM, $D_{\text {MAX }}$ AND HIGH END TAIL, $D_{\text {HIGH }}$, OF THE SLOWER DIFFUSING PEAK (NUMBER 1 , FIGURE 5.2) OF THE TOLUENE DIFFUSION SPECTRUM OBTAINED FOR A FRESH 40 WT\% TOLUENE SAMPLE.

FIGURE 5.4. PLOT OF THE PRODUCT $D \Delta$ AS A FUNCTION OF $\Delta$ FOR THE LOW END TAIL, $D_{\text {LOW }}$ MAXIMUM, $D_{\text {MAX }}$ AND HIGH END TAIL, $D_{\text {HIGH }}$, OF THE MIDDLE PEAK (FIGURE 5.2, NUMBER 2) OF THE TOLUENE DIFFUSION SPECTRUM OBTAINED FOR A FRESH 40 WT\% TOLUENE SAMPLE.

FIGURE 5.5. FAST DIFFUSING PORTION OF THE ECHO ATTENUATION OF THE OIL SIGNAL ABOVE $\Delta_{\text {SWITCH }}$ FOR EMULSION SAMPLES PREPARED WITH TOLUENE BETWEEN 5 AND 55 WT\%. LINES TO GUIDE EYES ONLY.

FIGURE 5.6. DIFFUSION ECHO ATTENUATION OF THE OIL SIGNAL ABOVE $\Delta_{\text {SWITCH }}$ (SINGLE EXPONENTIAL DECAY) FOR EMULSION SAMPLES PREPARED WITH TOLUENE BETWEEN 5 AND 70 WT\%. 
FIGURE 5.7. MOTIONAL AVERAGING OF THE OIL DIFFUSION VERSUS OIL CONCENTRATION BETWEEN 5 AND 70 WT\% FOR FRESH TOLUENE/TRITON X100/WATER EMULSIONS.

FIGURE 5.8. DIFFUSION SPECTRA OF THE OIL SIGNAL FOR EMULSIONS PREPARED WITH TOLUENE BETWEEN 15 AND $65 \mathrm{WT} \%$ TOLUENE, $\Delta=40$ MS.

FIGURE 5.9. PLOT OF THE PRODUCT $D \Delta$ AS A FUNCTION OF $\Delta$ FOR THE LOW END TAIL, $D_{\text {LOW }}$, MAXIMUM, $D_{\text {MAX }}$ AND HIGH END TAIL, $D_{\text {HIGH }}$, OF THE PEAK ASSOCIATED WITH SLOWEST DIFFUSION IN THE TOLUENE DIFFUSION SPECTRUM OBTAINED FOR A FRESH 65 WT\% TOLUENE SAMPLE. 125

FIGURE 5.10. DIFFUSION SPECTRA OF THE OIL SIGNAL FOR EMULSIONS PREPARED WITH TOLUENE BETWEEN 15 AND $65 \mathrm{WT} \%, \Delta=60 \mathrm{MS}$. THE FAST DIFFUSING PEAKS FOR BOTH THE 15 AND 25 WT\% OIL SAMPLES HAVE BEEN CUT FOR BETTER VISUALIZATION OF THE REMAINING PEAKS.

FIGURE 5.11. NMR SWITCHING TIME $\Delta_{\text {SWITCH }}$ VERSUS OIL CONCENTRATION FOR FRESH TOLUENE/TRITON X-100/WATER EMULSIONS PREPARED BETWEEN 5 AND 70 WT\% TOLUENE.

FIGURE 5.12. HOLE NUCLEATION IN AN O/W EMULSION. 135

FIGURE 5.13. TIME-DEPENDENT NORMALISED DIFFUSION COEFFICIENT FOR THERMALLY POLARISED XENON GAS IMBIBED IN RANDOMLY PACKED SPHERICAL GLASS BEADS TAKEN FROM REFERENCE [111]. BEADS HAVE UNIFORM DIAMETERS, B, OF 0.5, 1, 2 AND 4 MM. THE GAS DIFFUSION COEFFICIENT IN THE POROUS MEDIUM IS NORMALISED TO THE GAS BULK DIFFUSION COEFFICIENT $\mathrm{D}_{0}$. THE NORMALISED DIFFUSION LENGTH IS DEFINED AS $(1 / \mathrm{B})\left(\mathrm{D}_{0} \mathrm{~T}\right)^{1 / 2}$

FIGURE 5.14. APPARENT DIFFUSION COEFFICIENT ASSOCIATED WITH THE RESTRICTED DIFFUSION OF THE LARGEST DROPLETS AS A FUNCTION OF THE NMR OBSERVATION TIME, $\triangle$, FOR A 40 WT\% TOLUENE EMULSION.

FIGURE 6.1. AMOUNT OF AQUEOUS PHASE EXPELLED AS A FUNCTION OF TIME FOR TOLUENE/TRITON X-100/WATER EMULSIONS PREPARED WITH TOLUENE BETWEEN 5 AND 70 WT\%.

FIGURE 6.2. AMOUNT OF AQUEOUS PHASE EXPELLED AS A FUNCTION OF TIME FOR TOLUENE/TRITON X-100/WATER EMULSIONS PREPARED WITH TOLUENE BETWEEN 40 AND 65 WT\%.

FIGURE 6.3. VOLUME FRACTION OF EXPELLED AQUEOUS PHASE NORMALISED TO THE LIMITING VALUE MEASURED AT T TTATIC AS A FUNCTION OF TIME FOR TOLUENE/TRITON X-100/WATER EMULSIONS PREPARED WITH TOLUENE BETWEEN 40 AND 65 WT\%

FIGURE 6.4. VOLUME FRACTION OF EXPELLED AQUEOUS PHASE NORMALISED TO THE LIMITING VALUE MEASURED AT T $T_{\text {STATIC }}$ AS A FUNCTION OF TIME FOR TOLUENE/TRITON X-100/WATER EMULSIONS PREPARED WITH TOLUENE BETWEEN 5 AND 20 WT\%.

FIGURE 6.5. PERCENTAGE OF EXPELLED AQUEOUS PHASE AS A FUNCTION OF TIME FOR TOLUENE/TRITON X-100/WATER EMULSIONS PREPARED WITH TOLUENE BETWEEN 25 AND 35 WT\%.

FIGURE 6.6. EMULSION PREPARED WITH 15 WT\% TOLUENE OBSERVED 48 H POST EMULSIFICATION. THIS PICTURE IS REPRESENTATIVE OF EMULSION SAMPLES BETWEEN 5 AND 35 WT\% TOLUENE.

FIGURE 6.7. DROPLET SIZE DISTRIBUTION OF THE TWO EMULSION LAYERS OBSERVED AFTER $\sim 40$ H FOR A PHASE SEPARATED EMULSION PREPARED WITH 20 WT\% TOLUENE AND MEASURED SEPARATELY USING SLS. 
FIGURE 6.8. DROPLET SIZE DISTRIBUTION OF A 40 WT\% TOLUENE EMULSION MEASURED AS A FUNCTION OF TIME OVER 5 DAYS USING SLS. THIS SIZE DISTRIBUTION IS REPRESENTATIVE OF EMULSIONS BETWEEN 5 AND 70 WT\% OIL

FIGURE 6.9. SLS VOLUME-BASED MEAN DROPLET DIAMETER PLOTTED AGAINST TIME FOR 15, 25, 40 AND 65 WT\% TOLUENE SAMPLES.

FIGURE 6.10. INVERSE OF THE SQUARE VOLUME-BASED MEAN DROPLET DIAMETER PLOTTED AGAINST TIME FOR 15, 25, 40 AND 65 WT\% TOLUENE SAMPLES. ... 154

FIGURE 6.11. SQUARE AND CUBIC VOLUME-BASED MEAN DROPLET DIAMETER PLOTTED AGAINST TIME FOR A 65 WT\% TOLUENE EMULSION BETWEEN 10 MIN AND $1 \mathrm{H}$ POST EMULSIFICATION.

FIGURE 6.12. ECHO ATTENUATION OF THE OIL SIGNAL FOR A 15 WT\% TOLUENE EMULSION FRESHLY EMULSIFIED AND AGED OF 15 DAYS (UPPER CONCENTRATED EMULSION ONLY); $\Delta=100 \mathrm{MS}$.

FIGURE 6.13. ECHO ATTENUATION OF THE OIL SIGNAL FOR A FRESHLY EMULSIFIED $40 \mathrm{WT} \%$ TOLUENE EMULSION, $\Delta=100 \mathrm{MS}$, A $40 \mathrm{WT} \%$ TOLUENE EMULSION AGED OF 1 MONTH, $\Delta=100$ MS AND THE EXPELLED AQUEOUS PHASE OF A 40 WT\% TOLUENE EMULSION AGED OF 1 MONTH FOR $\Delta$ BETWEEN 6.3 MS AND $3 \mathrm{~s}$.

FIGURE 6.14. DIFFUSION COEFFICIENTS OF THE OIL SIGNAL MEASURED IN THE EXPELLED AQUEOUS PHASE OF AGED EMULSIONS PREPARED WITH TOLUENE BETWEEN 15 AND 60 WT\% FOR $\triangle$ BETWEEN 6.3 MS AND $3 \mathrm{~S}$

FIGURE 6.15. POSITION OF A PHASE SEPARATED EMULSION SAMPLE WITH RESPECT TO THE SPECTROMETER DETECTION COIL UPON PFG-NMR AGEING EXPERIMENTS.

FIGURE 6.16. DIFFUSION SPECTRA OF THE OIL SIGNAL FOR A 15 WT\% TOLUENE EMULSION OVER $70 \mathrm{H}$,

FIGURE 6.17. DIFFUSION SPECTRA OF THE OIL SIGNAL BETWEEN $1 \times 10^{-14}$ AND $1 \times$ $10^{-10} \mathrm{M}^{2} \mathrm{~s}^{-1}$ FOR A $15 \mathrm{WT} \%$ TOLUENE EMULSION OVER $70 \mathrm{H}, \Delta=60 \mathrm{MS} . . . . . . .163$

FIGURE 6.18. DIFFUSION SPECTRA OF THE OIL SIGNAL BETWEEN $3 \times 10^{-14}$ AND $2 \times$ $10^{-10} \mathrm{M}^{2} \mathrm{~s}^{-1}$ FOR A $25 \mathrm{WT} \%$ TOLUENE EMULSION BETWEEN 35 AND $70 \mathrm{H}, \Delta=60$ MS.

FIGURE 6.19. DIFFUSION SPECTRUM OF THE OIL SIGNAL FOR A 40 WT\% TOLUENE EMULSION OVER $70 \mathrm{H}$, 165

FIGURE 6.20. DIFFUSION SPECTRA OF THE OIL SIGNAL FOR A 65 WT\% TOLUENE EMULSION OVER $70 \mathrm{H}$,

FIGURE 6.21. NMR SWITCHING TIME BETWEEN A MULTIEXPONENTIAL AND A SINGLE EXPONENTIAL DECAY AS A FUNCTION OF EMULSION'S AGE FOR TOLUENE CONCENTRATION OF 15, 25, 40 AND 65 WT\%

FIGURE 6.22. NMR SWITCHING TIME VERSUS SLS MEAN DROPLET DIAMETER WITHIN THE FIRST 6 HOURS FOLLOWING EMULSIFICATION FOR AN EMULSION PREPARED WITH 65 WT\% TOLUENE.

FIGURE 6.23. NMR SWITCHING TIME VERSUS SLS MEAN DROPLET DIAMETER WITHIN THE FIRST 4 HOURS FOLLOWING EMULSIFICATION FOR AN EMULSION PREPARED WITH 40 WT\% TOLUENE.

FIGURE 6.24. NMR SWITCHING TIME AND SLS MEAN DROPLET DIAMETER VERSUS TIME FOR AN EMULSION PREPARED WITH 40 WT\% TOLUENE.

FIGURE 6.25. NMR SWITCHING TIME AND SLS MEAN DROPLET DIAMETER VERSUS TIME FOR A 15 AND 25 WT\% OIL EMULSIONS. 
FIGURE 6.26. MOTIONAL AVERAGING OF THE OIL DIFFUSION PLOTTED AGAINST TIME, CALCULATED BY AN INVERSE LAPLACE TRANSFORM FOR EMULSIONS PREPARED WITH 15, 25, 40 AND 65 WT\% TOLUENE, $\Delta \geq \Delta_{\text {SWITCH }} \ldots \ldots \ldots \ldots \ldots . . . . . . . .175$

FIGURE 7.1. DROPLET SIZE DISTRIBUTIONS OF EMULSIONS BETWEEN 2.5 AND 70 WT\% P-XYLENE USING SLS. .184

FIGURE 7.2. SIZE DISTRIBUTIONS OF THE EXPELLED AQUEOUS PHASE OF AGED EMULSIONS PREPARED WITH 20, 35 AND 40 WT\% P-XYLENE USING DLS. .... 187

FIGURE 7.3. AMOUNT OF EXPELLED AQUEOUS PHASE AS A FUNCTION OF TIME FOR $P$ XYLENE/TRITON X-100/WATER EMULSIONS PREPARED WITH $P$-XYLENE BETWEEN 2.5 AND $70 \mathrm{WT} \%$.

FIGURE 7.4. VOLUME FRACTION OF EXPELLED AQUEOUS PHASE NORMALISED TO THE LIMITING VALUE MEASURED AT T TTATIC AS A FUNCTION OF TIME FOR $P-$ XYLENE/TRITON X-100/WATER EMULSIONS PREPARED WITH P-XYLENE BETWEEN 2.5 AND 5 WT\%.

FIGURE 7.5. AMOUNT OF EXPELLED AQUEOUS PHASE AS A FUNCTION OF TIME FOR PXYLENE/TRITON X-100/WATER EMULSIONS PREPARED WITH P-XYLENE BETWEEN 7.5 AND 23 WT\%.

FIGURE 7.6. VOLUME FRACTION OF EXPELLED AQUEOUS PHASE NORMALISED TO THE LIMITING VALUE MEASURED AT T TTATIC AS A FUNCTION OF TIME FOR $P$ XYLENE/TRITON X-100/WATER EMULSIONS PREPARED WITH $P$-XYLENE BETWEEN 10 AND 20 WT\%.

FIGURE 7.7. VOLUME FRACTION OF EXPELLED AQUEOUS PHASE NORMALISED TO THE LIMITING VALUE MEASURED AT T TTATIC AS A FUNCTION OF TIME FOR PXYLENE/TRITON X-100/WATER EMULSIONS PREPARED WITH $P$-XYLENE BETWEEN 25 AND 35 WT\%.

FIGURE 7.8. VOLUME FRACTION OF EXPELLED AQUEOUS PHASE NORMALISED TO THE LIMITING VALUE MEASURED AT $\mathrm{T}_{\text {STATIC }}$ AS A FUNCTION OF TIME FOR $P$ XYLENE/TRITON X-100/WATER EMULSIONS PREPARED WITH P-XYLENE BETWEEN 40 AND 55 WT\%

FIGURE 7.9. 2D CONFOCAL MICROSCOPY OPTICAL CROSS-SECTIONS $256 \times 256(\times 100$ OBJECTIVE, ZOOM FACTOR 4) OF FRESH 65 WT\% P-XYLENE EMULSIONS LABELLED WITH NILE RED (A) AND 4-(4-METHOXYBENZYLAMINO)-7NITROBENZOFURAZAN (B). SNAPSHOT (C) RESULTS FROM THE MIXING OF THE RED FLUORESCING (A) AND THE GREEN FLUORESCING (B) EMULSIONS. 195

FIGURE 7.10. 2D CONFOCAL MICROSCOPY OPTICAL CROSS-SECTIONS 256×256 ( $\times 100$ OBJECTIVE, ZOOM FACTOR 4) OF FRESH 40 WT\% OCTANE EMULSIONS LABELLED WITH BODIPY 665/676 (A) AND BODIPY 493/503 (B). SNAPSHOT (C) RESULTS FROM THE MIXING OF THE RED FLUORESCING (A) AND THE GREEN FLUORESCING (B) EMULSION.

FIGURE 7.11. CRYO-SEM IMAGE OF A FRESHLY EMULSIFIED $40 \mathrm{WT} \%$ P-XYLENE EMULSION $(\times 20000)$ 198

FIGURE 7.12. CRYO-SEM IMAGE OF A 15 WT\% P-XYLENE EMULSION AGED FOR $4 \mathrm{H}$ $(\times 5000)$.

FIGURE 7.13. CRYO-SEM IMAGE OF A $40 \mathrm{WT} \%$ P-XYLENE EMULSION AGED FOR 9 DAYS $(\times 2000)$

FIGURE 7.14. CRYO-SEM IMAGE OF A FRESHLY EMULSIFIED 40 WT\% OCTANE EMULSION $(\times 5000)$.

FIGURE 7.15. CRYO-SEM IMAGE OF A FRESHLY EMULSIFIED 40 WT\% OCTANE EMULSION $(\times 10000)$. 
FIGURE 7.16. DIFFUSION ECHO ATTENUATION OF THE OIL SIGNAL FOR A FRESH 40 WT\% TOLUENE EMULSION OBSERVED AT $\Delta=40$ MS (MULTIEXPONENTIAL

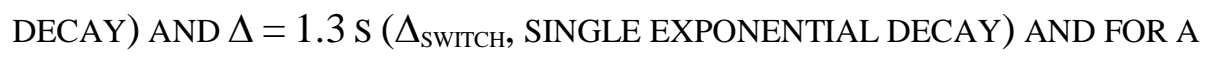
FRESH 40 WT\% $P$-XYLENE EMULSION OBSERVED AT $\Delta=40$ MS (MULTIEXPONENTIAL DECAY) AND $\Delta=1.5 \mathrm{~s}\left(\Delta_{\text {SWITCH }}\right.$, SINGLE EXPONENTIAL DECAY).

FIGURE 7.17. DIFFUSION SPECTRA OF THE OIL SIGNAL AS A FUNCTION OF $\Delta$ FOR A FRESH 40 WT\% $P$-XYLENE EMULSION.

FIGURE 7.18. PLOT OF THE PRODUCT $D \Delta$ AS A FUNCTION OF $\Delta$ FOR THE LOW END TAIL $D_{\text {LOW }}$ MAXIMUM $D_{\text {MAX }}$ AND HIGH END TAIL $D_{\text {HIGH }}$ OF THE MIDDLE PEAK IN THE $P$-XYLENE DIFFUSION SPECTRA OBTAINED FOR A FRESH 40 WT\% OIL EMULSION. 203

FIGURE 7.19. PLOT OF THE PRODUCT $D \Delta$ AS A FUNCTION OF $\Delta$ FOR THE LOW END TAIL $D_{\text {LOW }}$ MAXIMUM $D_{\text {MAX }}$ AND HIGH END TAIL $D_{\text {HIGH }}$ OF THE PEAK ASSOCIATED WITH THE SLOWEST DIFFUSION IN THE P-XYLENE DIFFUSION SPECTRA OBTAINED FOR A FRESH 40 WT\% OIL EMULSIONS

FIGURE 7.20. NMR SWITCHING TIME $\Delta_{\text {SWITCH }}$ VERSUS OIL CONCENTRATION FOR FRESH $P$-XYLENE AND TOLUENE-BASED EMULSIONS BETWEEN 2.5 AND $70 \mathrm{WT} \%$

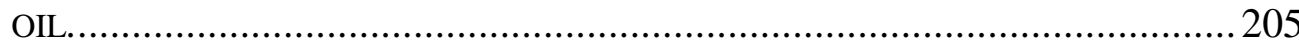

FIGURE 7.21. DIFFUSION COEFFICIENTS OF THE OIL SIGNAL DETECTED IN THE EXPELLED AQUEOUS PHASE OF AGED EMULSIONS PREPARED WITH $P$-XYLENE BETWEEN 15 AND 40 WT\% FOR $\triangle$ BETWEEN 6.3 MS AND $3 \mathrm{~S}$

FIGURE 7.22. MOTIONAL AVERAGING OF THE OIL DIFFUSION VERSUS OIL CONCENTRATION FOR TOLUENE AND P-XYLENE-BASED EMULSIONS. ............208

FIGURE 7.23. DROPLET SIZE DISTRIBUTIONS OF A $50 \mathrm{WT} \%$ P-XYLENE EMULSION AS A FUNCTION OF TIME USING SLS.

FIGURE 7.24. AMOUNT OF EXPELLED AQUEOUS PHASE AS A FUNCTION OF TIME TAKEN FROM REFERENCE [49], FOR OCTANE/TRITON X-100/WATER EMULSIONS PREPARED WITH TRITON X-100 BETWEEN 8 AND 12 WT\% WHILE MAINTAINING THE OCTANE-TO-TRITON X-100 WEIGHT RATIO TO 5:1. 


\section{INDEX OF TABLES}

TABLE 5.1. DIFFUSION COEFFICIENT OF TOLUENE MOLECULES DISSOLVED IN THE AQUEOUS CONTINUOUS PHASE AS A FUNCTION OF OIL CONCENTRATION BETWEEN 5 AND 70 WT\% OBTAINED USING AN INVERSE LAPLACE TRANSFORM.

121

TABLE 5.2. MEASUREMENT OF THE ZETA POTENTIAL OF EMULSIONS PREPARED WITH $5,15,40$ AND 55 WT\% TOLUENE.

TABLE 5.3. ZETA POTENTIAL OF A 15 WT\% TOLUENE EMULSION UPON ADDITION OF NACL AT VARIOUS CONCENTRATIONS BETWEEN $8.3 \times 10^{-5}$ AND $4.1 \times 10^{-3} \mathrm{MOL}$ $\mathrm{L}^{-1}$ 131

TABLE 5.4. NMR SWITCHING TIME BETWEEN A SINGLE AND A MULTIEXPONENTIAL DECAY OF THE ECHO ATTENUATION OF THE OIL SIGNAL FOR A 15 WT\% TOLUENE EMULSION UPON ADDITION OF NACL BETWEEN $8.3 \times 10^{-5}$ AND $4.1 \times$ $10^{-3} \mathrm{MOL} \mathrm{L}^{-1}$

TABLE 6.1. CHARACTERISTIC TIMES AND AMOUNT OF EXPELLED AQUEOUS PHASE DURING THE MACROSCOPIC PHASE SEPARATION PROCESS FOR EMULSIONS BETWEEN 40 AND 65 WT\% TOLUENE.

TABLE 6.2. CHARACTERISTIC TIMES OF THE PHASE SEPARATION PROCESS AND MAXIMUM EXPELLED AQUEOUS PHASE FOR EMULSIONS BETWEEN 5 AND 20 WT\% TOLUENE.

TABLE 6.3. CHARACTERISTIC TIMES OF THE PHASE SEPARATION PROCESS AND MAXIMUM EXPELLED AQUEOUS PHASE FOR EMULSIONS BETWEEN 25 AND 35 WT\% TOLUENE.

TABLE 6.4. TIME OF VISUALISATION OF A DROPLET SIZE GRADIENT UPON VARYING OIL CONCENTRATION BETWEEN 10 AND 35 WT\%.

TABLE 6.5. CHARACTERISTIC TIMES AND SLS VOLUME BASED MEAN DROPLET DIAMETER MEASURED AFTER 6 H FOR EMULSIONS PREPARED WITH 15, 25, 40 AND 65 WT\% TOLUENE.

TABLE 6.6. RATE OF COALESCENCE DURING THE FIRST 6 HOURS FOLLOWING EMULSIFICATION FOR EMULSIONS PREPARED WITH 15, 25, 40 AND 65 WT\% TOLUENE.

TABLE 6.7. RIPENING RATES BASED ON THE LINEAR FITS FOR STANDARD AND ENHANCED OSTWALD RIPENING MECHANISMS.

TABLE 7.1. SLS VOLUME-BASED MEAN DROPLET DIAMETER FOR EMULSIONS PREPARED WITH $P$-XYLENE OR TOLUENE BETWEEN 2.5 AND 70 WT\%.

TABLE 7.2. CHARACTERISTIC TIMES OF THE MACROSCOPIC PHASE SEPARATION PROCESS FOR THE FOUR CONCENTRATION DOMAINS AND THE THREE TRANSITION CONCENTRATIONS OF THE $P$-XYLENE/TRITON X-100/WATER SYSTEM BETWEEN 2.5 AND 60 WT\% P-XYLENE. 


\section{LIST OF PUBLICATIONS}

The contents of the chapters 4 and 5 have been published in the following manuscript:

N. Malassagne-Bulgarelli, K. M. McGrath, Soft Matter 2009, 5(23), 4804-4813. 


\section{Chapter 1 Introduction}

An emulsion is a heterogeneous system consisting of at least one immiscible liquid dispersed in another in the form of droplets (Figure 1.1), having diameters in the range $\sim 0.05-100 \mu \mathrm{m}$. Generally, the two immiscible liquids are classified as oil and water and emulsions may be either oil droplets dispersed in water $(\mathrm{O} / \mathrm{W})$ or water droplets dispersed in oil (W/O). Such systems are thermodynamically unstable and require the addition of a third component called an emulsifier, such as a surface-active agent, finely divided solids, proteins or polymers to increase their stability.
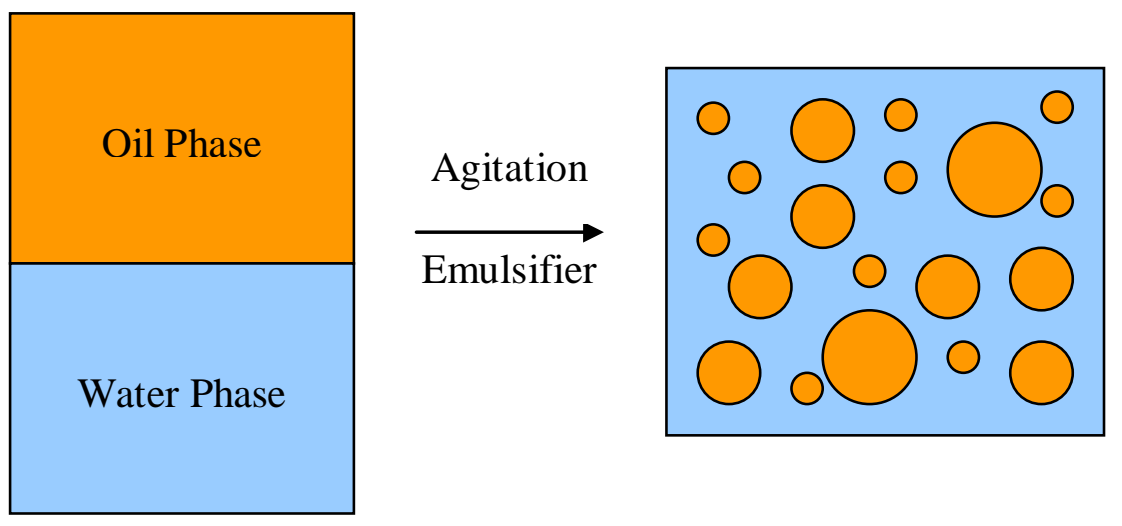

Figure 1.1. Formation of an oil-in-water $(\mathrm{O} / \mathrm{W})$ emulsion.

The droplets in an emulsion reside in the colloidal size range; hence the high surface area-to-volume ratios lead to some distinctive physicochemical properties compared to the unmixed individual components. Moreover, an emulsion system enables several immiscible fluids to be incorporated into the same matrix. These characteristics are the reason why emulsions are encountered in a very wide range of industries [1]. Examples are food emulsions in the form of mayonnaise or salad creams, cosmetics such as skin-care creams and sunscreens, agrochemicals in the form of self-emulsifiable oils which produce emulsions on dilution with water, pharmaceuticals in the form of multiple emulsions, paints such as latex emulsions etc. Moreover, bitumen emulsions are prepared stable in the containers but when applied to the road chippings they must coalesce to form a uniform film of 
bitumen. In the oil industry, many crude oils contain water droplets that must be removed by coalescence followed by separation.

However, because of their intrinsic instability the formulation of an emulsion with specific physicochemical properties remains elusive and to date only empirical approaches are used. Therefore, it is necessary to improve our basic understanding about the relationship between emulsion composition, microstructure and dynamics in order to achieve greater control and predictability. In this thesis is reported the investigation of emulsions in the ternary model system oil/Triton X-100/water where the oil is one of toluene, $p$-xylene or octane. The dynamics of these three oil systems are of particular interest. The occurrence of an oil exchange process between oil droplets that is independent of macroscopic destabilisation processes was evidenced. The rate at which oil exchange occurs is affected by the oil concentration, the oil used in emulsification, the addition of an electrolyte or by ageing the emulsion. The time constant for oil exchange is profoundly dependent on the chemical and physical characteristics of the thin interfacial film. The exchange occurs either by direct droplet contact (permeation via transient holes in the thin interfacial film) or reversible coalescence (a fusion-fission mechanism) or a combination of the two.

The thesis is divided into eight chapters. In addition to an introduction about emulsion physicochemical characteristics and the experimental techniques used to characterize the samples, evidence for the occurrence of oil exchange in the toluene/Triton X-100/water ternary system as well as the factors affecting the time constant for this process including oil concentration, emulsion age, addition of salt and nature of the oil are reported in four chapters. In the last chapter, the work performed in the project is summarised and future work to improve our understanding of emulsion microstructure and dynamics is suggested. 


\section{Chapter 2 Emulsion properties}

\subsection{Introduction}

Liquid-liquid dispersions may be classified according to the size of the dispersed phase as shown in Figure 2.1 [2].

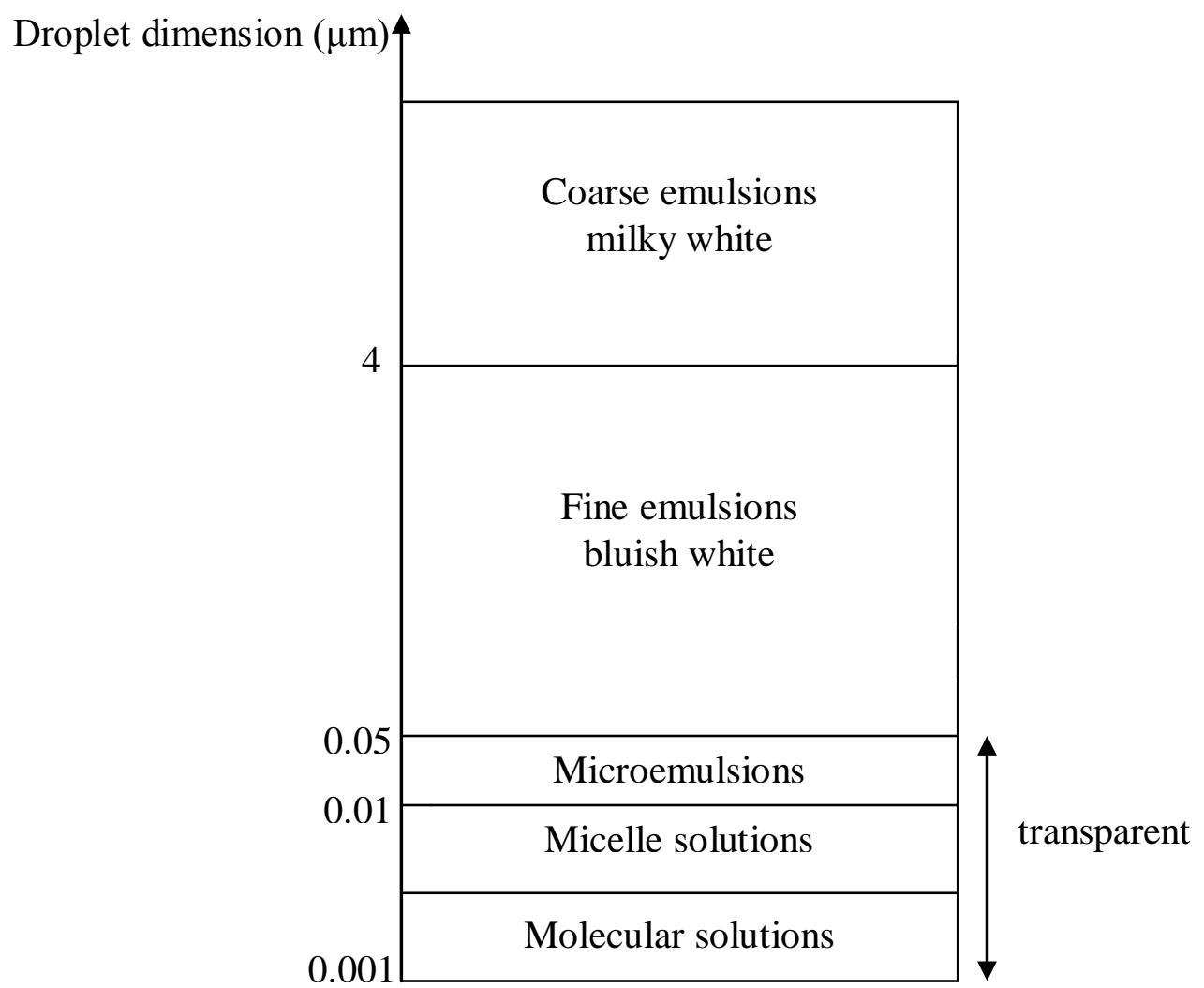

Figure 2.1. Droplet dimension for liquid-liquid dispersed systems.

Macroemulsions, commonly abbreviated emulsions, possess a broad distribution of droplet sizes in the $\sim 0.05-100 \mu \mathrm{m}$ range. They are characterized by a milky or bluish white colour due to light scattering. They are not thermodynamically stable due to the inherent immiscibility of the two phases resulting in a high interfacial tension. Therefore, they require the addition of an emulsifier located at the interface to decrease the interfacial tension by maintaining repulsive forces between the droplets. Energy input is needed for emulsions to be formed from the unmixed components. However, destabilization occurs over time via several mechanisms. 
By contrast, microemulsions have structural droplet sizes in the $\sim 10-50 \mathrm{~nm}$ range. They are thermodynamically stable due to the low interfacial tension between the two phases. Additionally, microemulsions are transparent since droplet sizes are generally much smaller than the wavelength of visible light. However, the distinction between emulsions and microemulsions based on droplet size has become questionable over the past decades with the identification of transparent non thermodynamically stable emulsions whose droplets are a few nanometres in size, defined as nanoemulsions.

\subsection{Emulsion formation}

\subsubsection{Surface activity}

In a bulk water or oil phase, each molecule is subjected to an equal attraction from its neighbouring molecules due to the van der Waals attractive forces. The net attractive force felt by each molecule in a bulk liquid is equal to zero because the van der Waals attractive forces are balanced, however when the bulk phase is mixed with another phase whose chemical structure and properties are different (e.g. oil and water), the net attractive force becomes positive (Figure 2.2). So as to lower the system energy as much as possible, the resulting interface is shaped so that the surface of contact between the two phases is minimized. As a result of immiscibility and gravity, water and oil form two layers one above another upon gentle mixing. 


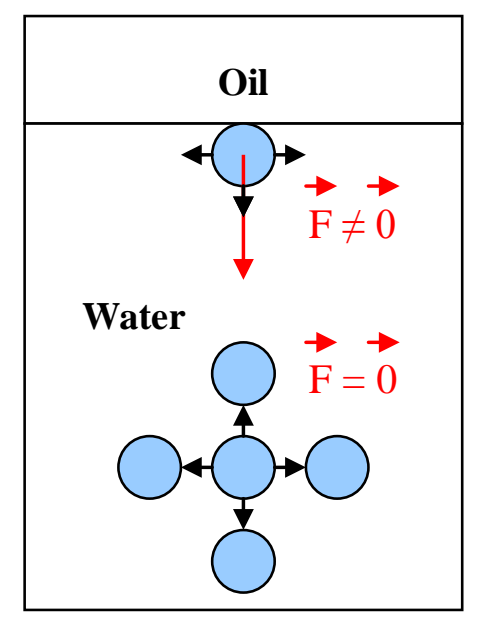

Figure 2.2. van der Waals forces in a bulk liquid. In the absence of an interface, the net attractive force $F$ felt by a molecule is equal to zero whereas in the presence of an interface, the net attractive force is positive.

When an emulsion is formed, there is a very large increase in interfacial surface area since one phase is dispersed as droplets in the other. To accomplish this, some energy is required to be added to the system. The so-called interfacial tension $\gamma$ is the work in $\mathrm{mN} \mathrm{m}^{-1}$ required to generate $1 \mathrm{~m}^{2}$ of surface expansion. The energy for emulsion formation, $\Delta G_{f o r m}$, is a function of the work performed to expand the interfacial area and the increase in configurational entropy according to the second law of thermodynamics (Eq.2.1).

$$
\Delta G_{\text {form }}=\Delta A \gamma-T \Delta S_{\text {config }}
$$

where $\Delta A$ is the increase in interfacial surface area, $\gamma$ the interfacial tension, $T$ the temperature and $\Delta S_{\text {config }}$ the configurational entropy. $\Delta S_{\text {config }}$ is a positive quantity since producing a large number of droplets is accompanied by an increase in configurational entropy. The work term $\Delta A \gamma$ is positive and larger than $-T \Delta S_{\text {config }}$ in most cases; consequently the energy of emulsion formation, $\Delta G_{f o r m}$, is a positive quantity. Emulsion formation is then non-spontaneous and emulsions are thermodynamically unstable. Moreover, the system minimizes the interfacial surface area, which results in one phase dispersed in the second in the form of spherical droplets. 


\subsubsection{Emulsifiers}

\subsubsection{Introduction}

In most cases, in the absence of emulsifier, energy input is not sufficient to stabilize an emulsion. Indeed, the droplets formed have such a high interfacial tension that the system reverts quickly to two layers, the lowest energy configuration. Hence, the emulsifier located at the interface between the two phases (Figure 2.3) plays a significant role by lowering the interfacial tension on the one hand, thus allowing emulsions to form more easily and on the other hand by providing a protective electrically charged and/or steric barrier against coalescence (section 2.3).

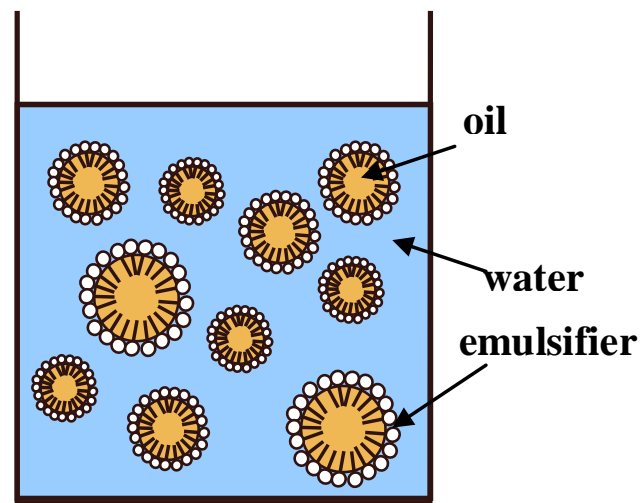

Figure 2.3. Location of the emulsifier at the oil/water interface in an $\mathrm{O} / \mathrm{W}$ emulsion (not to scale).

The emulsifier is oriented such that its hydrophobic residue dips into the oil phase while the hydrophilic group is in water. The thickness of the interfacial film is about $10 \mathrm{~nm}$ for most emulsions and this includes a large number of oil and water molecules. To attain stability emulsifiers need to cover the surface of the droplets at least partially and remain firmly adsorbed. The amount of emulsifier required for a given emulsion is highly dependent on the chemical affinity of the emulsifier for both the water and the oil phases. Most emulsifiers used are amphiphilic, i.e. they possess both hydrophilic and hydrophobic properties. 


\subsubsection{Classification}

A rather arbitrary classification of emulsifiers may be made [3] as follows

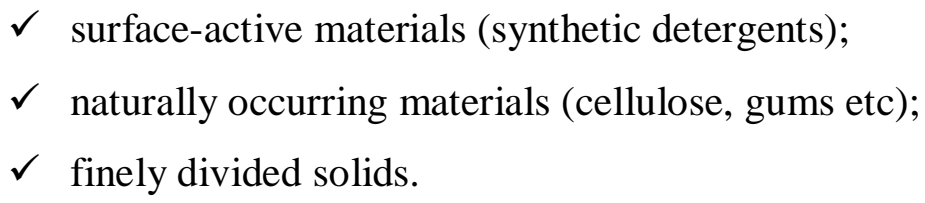

Most of the emulsifiers used in industry belong to the surface-active agent group. For this reason, Schwartz and Perry [4] proposed a subcategorisation of this group based on the ionic properties of the hydrophilic group in the molecule.

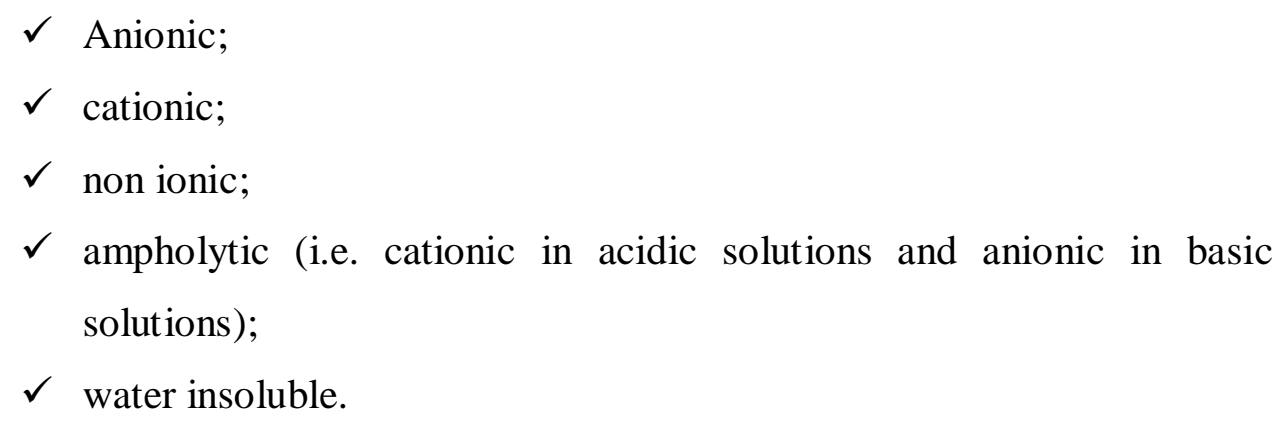

\subsubsection{Choice of the emulsifier}

The efficiency of an emulsifier may be considered in terms of cost and type of emulsions required. Indeed, an emulsifier is more efficient to stabilize $\mathrm{O} / \mathrm{W}$ emulsions if it possesses a higher solubility in water whereas an emulsifier more soluble in oil is recommended to form a W/O emulsions. This generalization is known as Bancroft's rule, which can be expressed as follows: the phase in which the emulsifier is more soluble is the outer phase. A successful qualitative approach with regard to the choice of emulsifier is due to Griffin [5]. He introduced the concept of "hydrophilic-lipophilic balance" or HLB. Each emulsifier is assigned a dimensionless number between 0 and 20 reflecting the fraction of hydrophilicity in the molecule. Emulsifiers whose HLB is between 0 and 9 are hydrophobic emulsifiers stabilizing W/O emulsions whereas a HLB between 11 and 20 is associated with hydrophilic emulsifiers in favour of $\mathrm{O} / \mathrm{W}$ 
emulsions. Emulsifiers with a HLB value of 10 are said to be balanced hydrophilically-lipophilically. Numerous methods have been proposed to determine the HLB number of an emulsifier but the most used are those from Davies [6] and Griffins [7] based on the composition and the chemical structure of the surface-active agent. It should be noted though that the HLB is of very limited predictive value.

\subsubsection{Mode of addition of the emulsifier}

The mode of addition of the emulsifier requires consideration when dealing with emulsions having dispersed phase volume fractions $>0.3$ [3]. It affects not only the type of the final emulsion but also the droplet size distribution. A variety of methods can be employed.

\section{1/ Agent-in-water method}

In this method, the emulsifying agent is dissolved directly in the water and the oil phase is then added while the mixture is stirred vigorously. This procedure makes $\mathrm{O} / \mathrm{W}$ emulsions directly; should a W/O emulsion be desired, the oil addition is continued until inversion takes place, i.e. the process whereby an $\mathrm{O} / \mathrm{W}$ emulsion changes to W/O or vice versa. Emulsions obtained this way are coarse with a wide droplet size distribution.

\section{2/ Agent-in-oil method}

In this method, the emulsifier is dissolved in the oil phase. The emulsion may then be formed in two ways

- $\quad$ by adding the mixture directly to the water. In this case, an $\mathrm{O} / \mathrm{W}$ emulsion forms spontaneously.

- By adding water directly to the mixture, slowly and in small quantities . In this case a W/O emulsion is formed. In order to produce an $\mathrm{O} / \mathrm{W}$ emulsion 
it is necessary to induce an inversion of the emulsion by the addition of further water.

\section{$\underline{\text { 3/ Alternate addition method }}$}

The water and oil are added alternately in small portions to the emulsifier. This method is particularly suitable for the preparation of food emulsions.

\subsubsection{Emulsification}

The energy input required to produce an emulsion can be introduced in many ways, although the most common method employs mechanical shear to achieve dispersion of the droplets. When a droplet undergoes shear, it distorts and breaks into smaller droplets (Figure 2.4). The droplets need to be stabilized as soon as they are formed or recombination takes place, i.e. there must be a reservoir of emulsifier present.

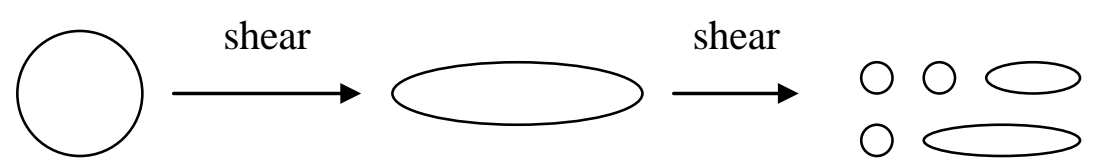

Figure 2.4. Shear undergone by a droplet during the emulsification process.

The shearing effect can be achieved by blade stirrers, stator-rotor dispersers, jet impact devices or by pressure-induced flow through the constriction of high pressure homogenisers (Figure 2.5). 


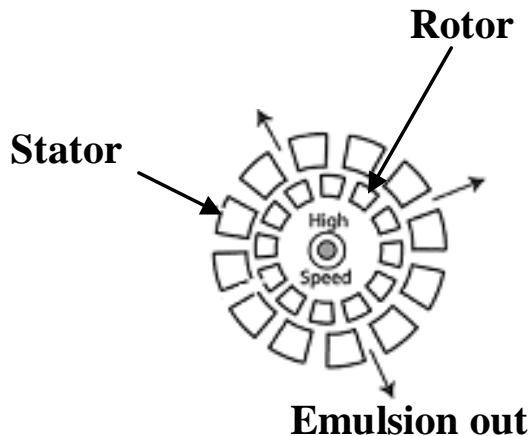

(a)
High Pressure

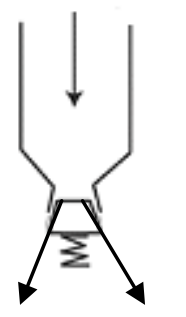

Emulsion out

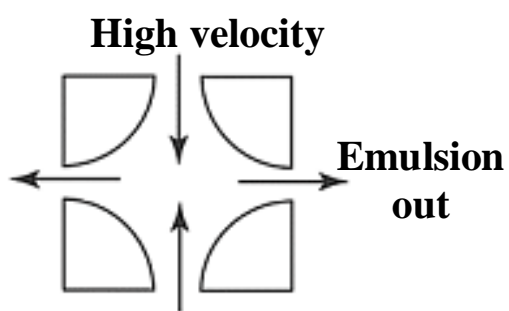

(c)

Figure 2.5. Emulsification techniques (a) rotor-stator disperser, (b) high pressure homogenizer and (c) jet impact device.

Rotor-stator dispersers rely on very high shear rates (speeds up to $22000 \mathrm{rpm}$ ) to accomplish droplet scission. Traditional rotor-stator devices utilise a cone-shaped rotor, whereas more recent designs can have concentric cylinders with complicated tooling to aid in droplet scission and turbulent flow. In high-pressure homogenizers, the emulsion components are forced through a small orifice at between 1000 and 5000 psi. Both rotor-stator devices and homogenizers can have single or multiple stages. In the jet impact system two liquid jets collide at high velocity thus forming small droplets. The resulting emulsion can be recycled through the unit to attain further droplet size reduction.

Depending on the range of viscosity and droplet size desired, several mechanical emulsification devices are used in industry [8] including

\footnotetext{
$\checkmark$ simple mixers;

$\checkmark$ turbine mixers;

$\checkmark$ colloid mills;

$\checkmark$ ball mills;

$\checkmark$ agitator ball mills;

$\checkmark$ perforated disk mills;

$\checkmark$ high-pressure homogenisers;

$\checkmark$ jet impact device;

$\checkmark$ ultrasound apparatus.
} 
Sometimes chemical energy is sufficient to produce an emulsion. This is referred to as "spontaneous emulsification". Heat of solvation or neutralization can cause localized flow and mixing (Marangoni effect) at the interface leading to emulsification. But the use of mechanical energy is still the preferred method for achieving emulsification.

\subsection{Emulsion stability}

\subsubsection{Introduction}

In order for an emulsion to be stable, the droplets need to exist as discrete entities uniformly dispersed in the continuous phase. When two droplets encounter, they coalesce unless there is a sufficiently high energy barrier to keep them apart. Several stabilization processes of colloidal systems have been presented but the central knowledge on colloidal stability is based on the Derjaguin-LandauVerwey-Overbeek theory abbreviated DLVO [9-11]. This theory explains the stability of colloidal systems by considering the balance between van der Waals attractive forces and electrostatic repulsive forces. Over the past decades, the consideration of other forces such as steric, hydration or hydrophobic have improved our basic understanding of colloidal stability [3].

\subsection{2. van der Waals (vdW) attractive forces}

van der Waals attractive forces constitute the universal phenomenon that similar molecules attract one another. They are considered to arise as a consequence of spontaneous fluctuations in the electron cloud in one molecule causing corresponding fluctuations in neighbouring molecules. These forces are separated into three types:

- Keesom or dipole/dipole interaction;

- Debye or dipole/induced dipole interaction;

- London or induced dipole/induced dipole interaction. 
In the case of emulsions, only the London-vdW forces are significant. Hamaker proposed a method to estimate the energy of interaction $G_{A}(h)$ (energy required to bring a pair of droplets from an infinite distance apart to some close surface-tosurface separation, $h$, involved in London-vdW forces in colloidal systems [12].

$$
G_{A}(h)=\frac{-A_{H}}{6} \times\left[\frac{2 a^{2}}{h^{2}+4 a h}+\frac{2 a^{2}}{h^{2}+4 a h+4 a^{2}}+\ln \left(\frac{h^{2}+4 a h}{h^{2}+4 a h+4 a^{2}}\right)\right]
$$

where $a$ is the radius of the interacting spheres, $h$ is the surface-to-surface separation and $A_{H}$ is the Hamaker constant which is dependent on the material properties.

In most cases $a<<h$ and the Hamaker equation simplifies to

$$
G_{A}(h)=\frac{-A_{H} a}{12 h}
$$

where $A_{H}$ is the Hamaker constant given, for an emulsion, by

$$
A_{H}=A_{H}^{0.5} \text { dispersed phase } \times A_{H}^{0.5} \text { continuous phase }
$$

In Figure 2.6 is shown the interaction energy-distance plot for two bare (i.e. no adsorbed emulsifier) droplets approaching each other. vdW attractive forces become significant from a separation distance of $\sim 2 \mathrm{~nm}$ and below. In the absence of repulsive forces, flocculation rapidly leads to large clusters and to irreversible coalescence (primary minimum). 


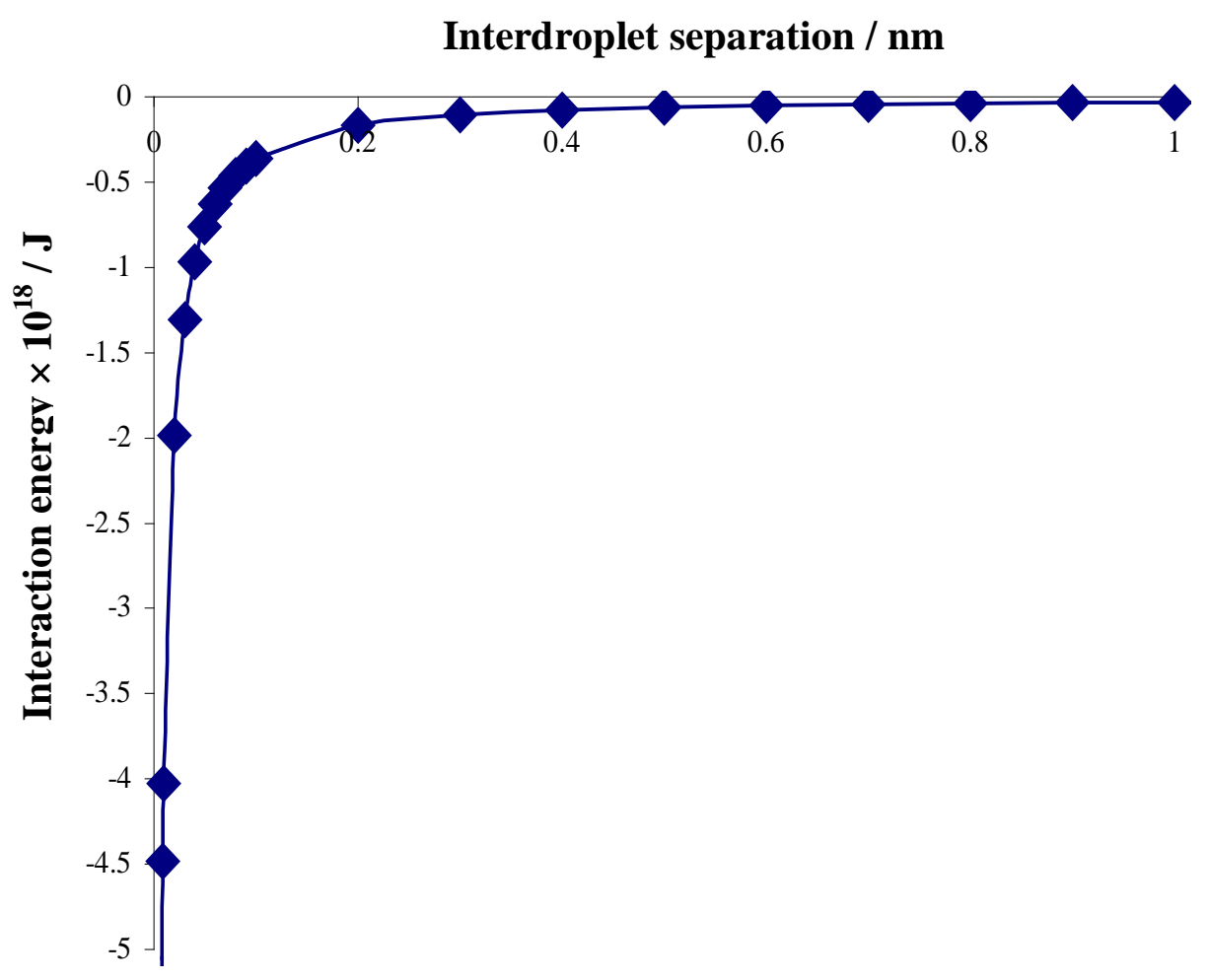

Figure 2.6. Interaction energy-distance plot for two droplets approaching each other in the absence of emulsifier, $A_{H}=3.7 \times 10^{-21} \mathrm{~J} ; a=0.133 \mu \mathrm{m}$.

\subsubsection{Repulsive forces}

In the presence of electrostatic and/or steric energy barriers, due to the presence of emulsifiers at the oil/water interface, emulsions can be kinetically stabilised. The nature of the repulsive forces depends on the type of emulsifier.

\subsubsection{Electrostatic repulsive forces}

In a colloidal system, the surface of the particles is charged either by adsorption of an ionic emulsifier, ions from the continuous phase or frictional contact [13] although the latter is not experimentally proven. Even in the presence of a nonionic surfactant, preferential adsorption of anions leads to a net negative charge on droplets. Indeed, anions have a greater tendency to adsorb than cations. This is because the latter are more strongly hydrated in bulk solution and so compete less effectively for surface sites than the more weakly hydrated anions. Even pure 
hydrocarbon oil droplets in pure distilled water carry a net negative charge presumably due to preferential adsorption of $\mathrm{OH}^{-}$ions over $\mathrm{H}_{3} \mathrm{O}^{+}$ions [14].

When the surface of the particles is charged, another layer of counter ions must be present to maintain electroneutrality (global and local). This is called the double layer and several models have been suggested such as the Helmholtz double layer [15], the Gouy-Chapman diffuse double layer [16, 17] and the Stern diffuse double layer [18], the latter is a compromise between the Helmholtz and Gouy models which have some deficiencies. The Stern model is exclusively used nowadays.

Figure 2.7 represents a schematic structure of the electrical double layer based on the Stern model for an O/W emulsion stabilised by an anionic surfactant accompanied by the corresponding interaction potential. Closest to the particle, the interaction potential of the particle decreases sharply due to the electrical compensation of the counter ions distributed in a compact layer bound to the particle called the Stern layer. To maintain electroneutrality, a diffuse layer contains the remaining counter ions where the interaction potential decreases exponentially to zero. The zeta potential measured by electrophoresis is located at the plane of shear (slipping plane). 


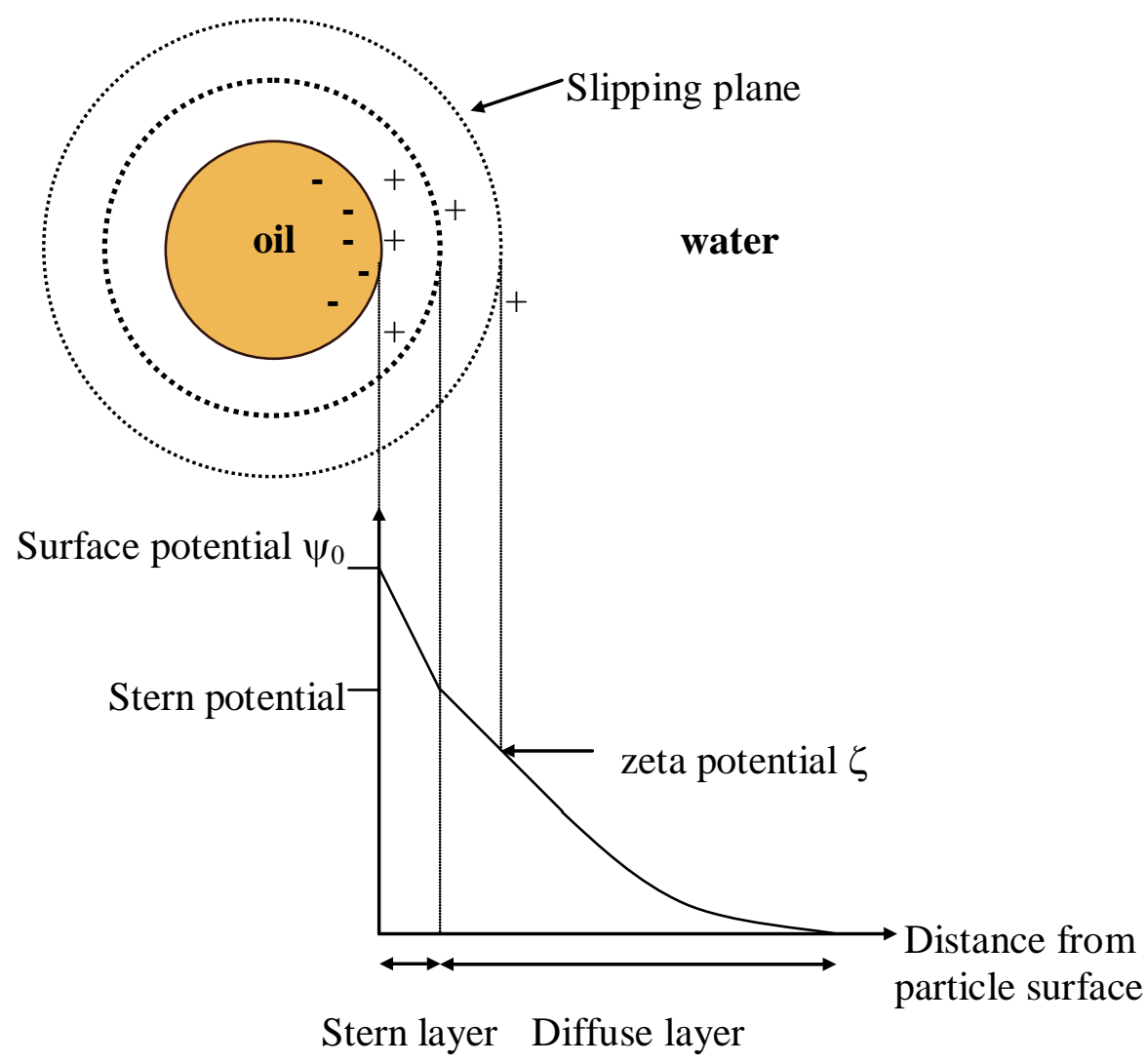

Figure 2.7. Structure of the electrical double layer at the oil/water interface with negatively charged adsorbed emulsifier.

As two identically charged particles approach one another, there is an increase in the concentration of small counter ions in the gap between the surfaces. The resulting increase in local osmotic pressure in the gap leads to a repulsive electrostatic force between the particles. This force is usually known as the double-layer repulsion, being derived from the overlap of electrical double-layers surrounding the two particles. For two charged spheres of radius $a$ in a medium of dielectric constant $\varepsilon_{r}$, the separation-dependent double-layer energy of interaction is given by

$$
G_{R}(h)=2 \pi \varepsilon_{r} \varepsilon_{0} a \psi_{0}^{2} \ln \left(1+e^{-\kappa h}\right)
$$

where $\varepsilon_{0}$ is the vacuum permittivity, $\psi_{0}$ the surface potential, $h$ the surface-tosurface separation and $\kappa^{-1}$ the Debye length which is an approximate measure of the thickness of the electrical double-layer.

Addition of electrolyte modifies the structure of the double layer. As the ionic strength and/or the valency of the electrolyte increases, the thickness of the 
double layer diminishes and the system becomes unstable as shown by the expression of the Debye length

$$
\kappa^{-1}=\sqrt{\frac{\varepsilon_{0} \varepsilon_{r} k_{B} T}{2 c N_{A} z^{2} e_{0}^{2}}}
$$

where $e_{o}$ is the elementary electric charge, $N_{A}$ is the Avogadro number, $z$ is the valency of the ions and $c$ is the electrolyte concentration.

The total interaction energy $G_{D L V O}(h)$ according to the DLVO theory results from the addition of the vdW attractive forces and electrostatic repulsive forces following Eq. 2.4.

$$
G_{D L V O}(h)=G_{A}(h)+G_{R}(h)
$$


In Figure 2.8 is shown the schematic representation of the interaction energydistance curve based on the DLVO theory.

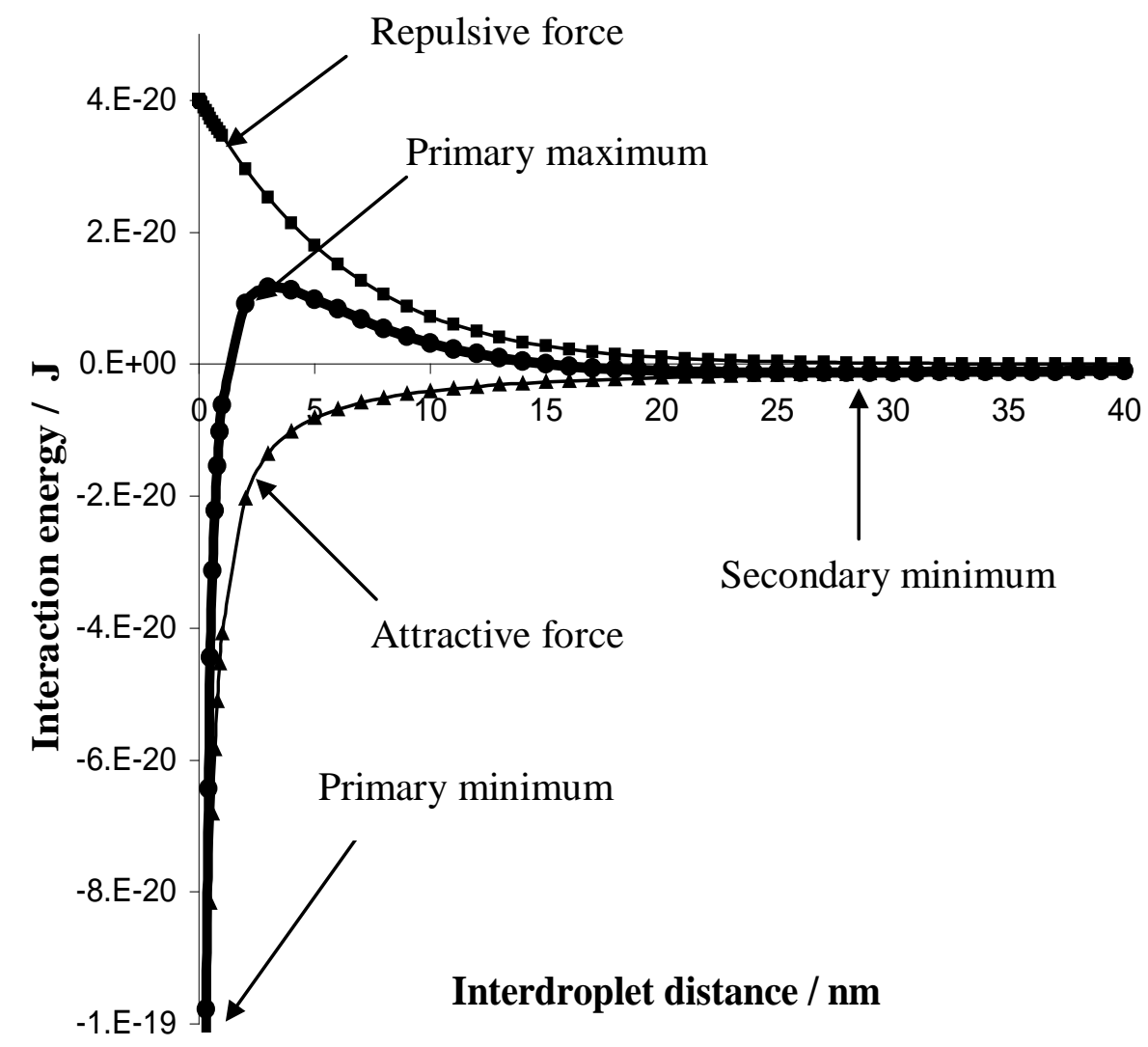

Figure 2.8. Interaction energy for two colloidal particles as a function of their distance of separation, for electrical double layers due to surface charge (squares, $G_{R}$ ), London-van der Waals dispersion forces (triangles, $G_{A}$ ), and the total interaction (bold line, $G_{D L V O}$ ) calculated with $A=3.7 \times 10^{-21} \mathrm{~J} ; a=0.133 \mu \mathrm{m} ; \varepsilon_{r}=78.54 \mathrm{~F} / \mathrm{m}$ and $\psi_{o}=0.01 \mathrm{~V}$.

At large interdroplet distances, attractive forces prevail which can result in a shallow secondary minimum where flocculation takes place (magnitude of a few $k T$ units [19]). At very short distances ( $<1 \mathrm{~nm})$, attractive forces again dominate which gives a primary minimum (magnitude large compared to thermal energy, several hundred $k T$ units) where irreversible coalescence occurs. At intermediate separations, repulsive electrostatic forces dominate resulting in a primary maximum whose height increases with increasing surface potential $\Psi_{0}$ and decreasing electrolyte concentration and valency. The primary maximum prevents close approach of the droplets and irreversible coalescence in the primary minimum. 


\subsubsection{Steric repulsive forces}

It is important to note that with a non-ionic emulsifier or in the case of W/O emulsions, electrostatic repulsive forces become very small. In the case of W/O emulsions, the very low dielectric constant of the oil phase prevents any electric double layer formation. However, it is still possible to stabilize these emulsions using oligomeric surfactants or high molecular weight polymers, this is steric stabilization since a physical barrier is present at the interface [20]. The droplets cannot get close enough to each other to fall into the primary minimum of the interaction energy - distance curve and coalesce providing that the polymer surface layer exceeds the 5-10 nm van der Waals interaction distance.

In the presence of steric stabilization, the interaction energy comprises two components following Eq. 2.5.

$$
G_{\text {steric }}(h)=G_{\text {mix }}(h)+G_{\text {elast }}(h)
$$

$G_{m i x}$ refers to the osmotic or mixing energy of interaction due to the interaction between the polymers and the solvent upon increasing the local polymer concentration when two particles interpenetrate [1] (Eq.2.6).

$$
G_{m i x}=k T \frac{4 \pi}{V_{1}} \phi_{2}^{2} N_{a v o g}\left(\frac{1}{2}-\chi\right)\left(3 R+2 \delta+\frac{h}{2}\right)\left(\delta-\frac{h}{2}\right)^{2}
$$

where $k$ is the Boltzmann constant, $T$ is the temperature, $V_{1}$ is the molar volume of the solvent, $\phi_{2}$ is the volume fraction of the polymer chains with an end-to-end distance $R$ and a thickness $\delta . \chi$ is the Flory-Huggins interaction parameter. When $\chi<0.5 G_{\text {mix }}$ is positive (unfavourable for polymer chains to interpenetrate in good solvents in which polymer chains are soluble and fully expanded), $\chi>0.5 G_{\text {mix }}$ is negative (thermodynamically favourable for polymer chains to interpenetrate in poor solvents in which polymer chains are not soluble and are compact), $\chi=0.5$ $G_{\text {mix }}=0$ ( $\theta$-condition).

$G_{\text {elast }}$ is the entropic, volume restriction or elastic energy of interaction resulting from the loss in configurational entropy of the chains on significant overlap. Entropy loss is unfavourable and therefore $G_{\text {elast }}$ is always positive. 
Hesselink and co-workers [21, 22] proposed the following expression for $G_{\text {elast }}$

$$
G_{\text {elast }}(h)=2 \sigma k T V(i, h)
$$

where $k$ is the Boltzmann constant, $T$ is the temperature, $\sigma$ is the concentration of hydrophobic tails at the particle surface (in grams of adsorbed polymer per weight of dispersed phase), $i$ is the average number of oligomer segments per chain, $h$ is the distance of separation of the particles and $V(i, h)$ is a repulsion energy term per chain calculated by numerical integration [3].

Figure 2.9 shows the schematic representation of the interaction energy-distance curve for colloidal particles undergoing vdW attractive forces and steric repulsion.

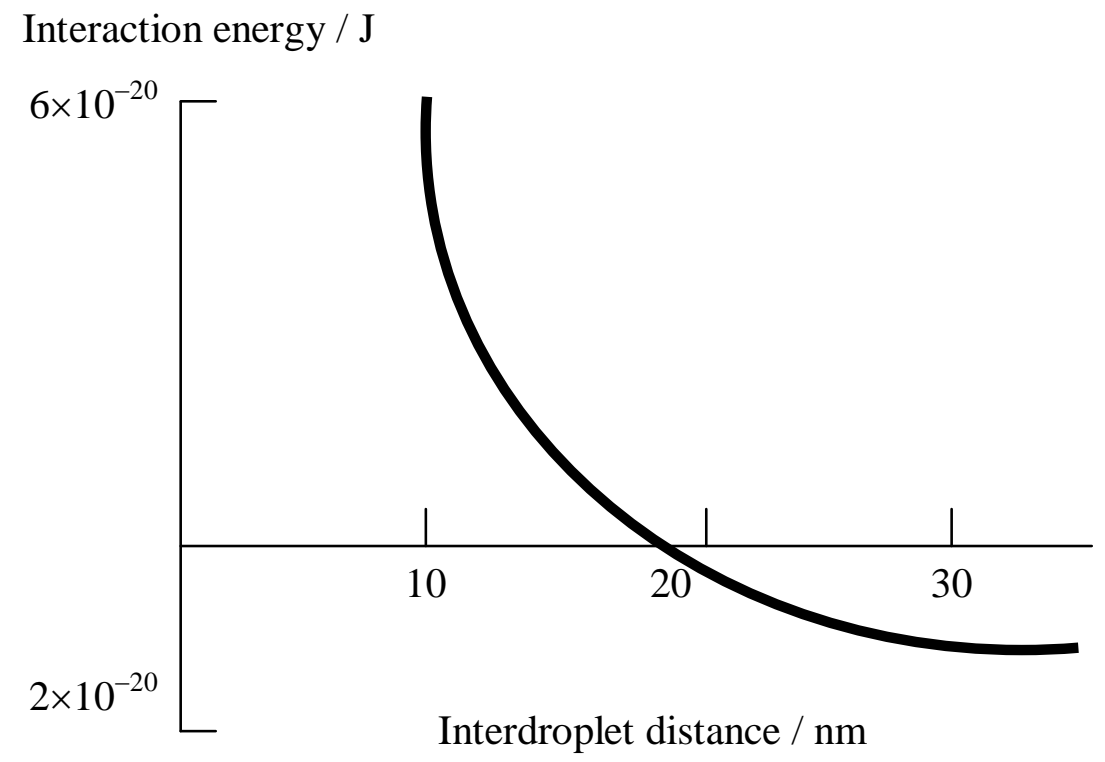

Figure 2.9. Total interaction energy for two colloidal particles as a function of their distance of separation due to steric repulsion and vdW attraction.

At large droplet-droplet distances, the vdW attraction predominates. At shorter distances in a good solvent, there is a very steep repulsion which effectively prevents further approach. At very close separations, even in poor solvents, the very strong repulsive contribution of $G_{\text {elast }}$ results in the absence of a primary minimum. In practice, full coverage of the particle surface is not achieved due to thermal fluctuations and interactions between polymer chains, which results in coalescence in a primary minimum. 


\subsubsection{Structural forces}

Additional influences on colloidal stability beyond those accounted for by the DLVO theory, such as hydration and hydrophobic effects have received considerable attention over the past decades [3].

\subsubsection{Hydration repulsive forces}

The structure of water (also true for any polar liquid) as the continuous phase in colloidal systems may have an impact on double-layer interactions. Indeed, the hydration repulsive forces also called solvation or structural forces have been assumed to arise from the strong hydrogen bonds between water molecules forming hydrating layers around ions at the surface of a particle [3]. Hydration forces result in thick oriented water layers producing short-range $(<5 \mathrm{~nm})$ repulsive effects by mutual interpenetration acting as a mechanical barrier between droplets [23, 24]. The magnitude of hydration forces depends on both the type and concentration of the electrolyte adsorbing at the particle surface.

\subsubsection{Hydrophobic attractive forces}

The attractive interaction between nonpolar molecules, such as hydrocarbons, in water has been measured to have the same range as, but is about an order of magnitude stronger than, the vdW attractive forces. Hydrophobic forces operate in the $0-10 \mathrm{~nm}$ range and decay exponentially with distance [24, 25]. The interaction energy can be expressed as

$$
G_{H}=a C_{0} D_{0} e^{\frac{-h}{D_{0}}}
$$

where $a$ is the particle radius, $C_{0}$ is a pre-exponential factor, $h$ is the interdroplet separation and $D_{0}$ is called the decay length.

Hydrophobic interactions are responsible for the very low solubility of hydrophobic molecules in water. It is recognized that the interaction involves the 
configurational rearrangement of water molecules as two hydrophobic species come together.

These interactions point out limitations in the DLVO theory to fully explain interaction forces in colloidal systems.

\subsection{Mechanisms of emulsion destabilization}

\subsubsection{Introduction}

Emulsions are not thermodynamically stable and phase separation into macroscopically isolated oil and water domains takes place to reach a state of lower energy. Emulsion destabilization occurs over a wide range of time scales from a few hours to several years depending on the composition, emulsification technique, formulation, concentration etc.

Emulsions are destabilised via five different mechanisms which can occur independently or combined

$\checkmark$ inversion;

$\checkmark$ creaming;

$\checkmark$ Ostwald ripening;

$\checkmark$ flocculation;

$\checkmark$ coalescence. 


\subsubsection{Inversion}

An emulsion is said to have inverted when it suddenly changes from $\mathrm{O} / \mathrm{W}$ to $\mathrm{W} / \mathrm{O}$ and vice versa (Figure 2.10).
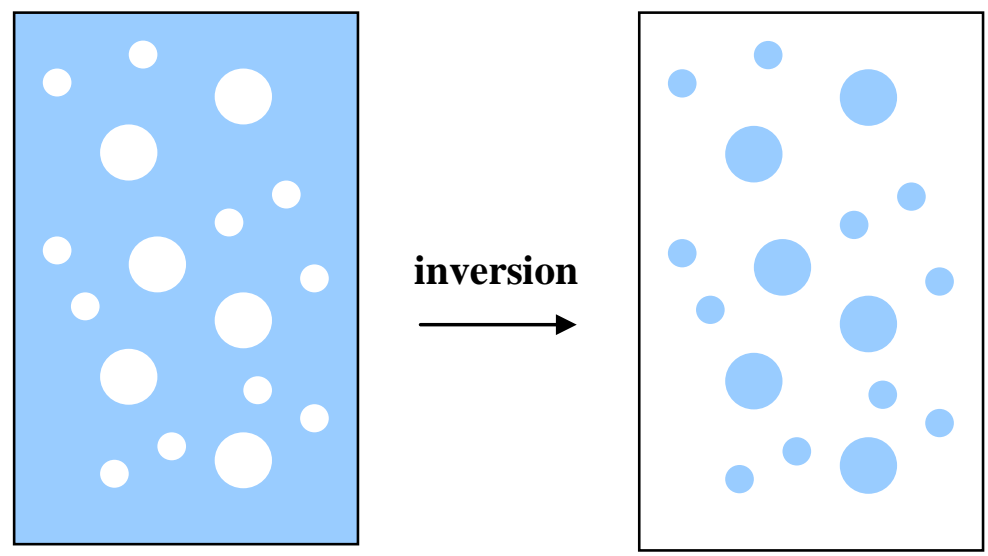

Figure 2.10. Inversion of an $\mathrm{O} / \mathrm{W}$ in a $\mathrm{W} / \mathrm{O}$ emulsion.

Inversion occurs upon a temperature variation. When the temperature is raised, the degree of hydration of the hydrophilic groups of the emulsifier is reduced and the emulsifier becomes less hydrophilic. Its HLB value decreases. So if an emulsion is $\mathrm{O} / \mathrm{W}$ type at lower temperatures, it can invert to a $\mathrm{W} / \mathrm{O}$ emulsion when the temperature is increased. Similarly, a W/O emulsion created at a high temperature can invert to an O/W type when the temperature drops. The temperature at which this inversion occurs is called the phase inversion temperature, PIT [26]. At this temperature, the hydrophilic and hydrophobic properties of the emulsifier are balanced.

Although the subject of considerable investigation, inversion is not well understood. Clowes [27] proposed that the process of inversion occurs by the system passing through a bicontinuous region where both oil and water are the continuous phase and both oil and water droplets are present. Sherman [28] has shown that the value of the dispersed phase volume fraction at inversion for a given emulsion varies with the emulsifier concentration and the nature of the emulsifier. In addition, Davies [29] found a linear dependence of the dispersed phase volume at inversion and the HLB of the emulsifier. 


\subsubsection{Creaming}

Creaming is not the breaking of the emulsion but the separation into two emulsions. A concentration gradient builds up and one part is richer in the dispersed phase, the other poorer than the original emulsion (Figure 2.11). The more concentrated emulsion is called the cream and is located either at the top $(\mathrm{O} / \mathrm{W})$ or the bottom $(\mathrm{W} / \mathrm{O})$. The latter behaviour refers to sedimentation. This destabilization process can usually be reversed, at least temporarily, by simple mixing.

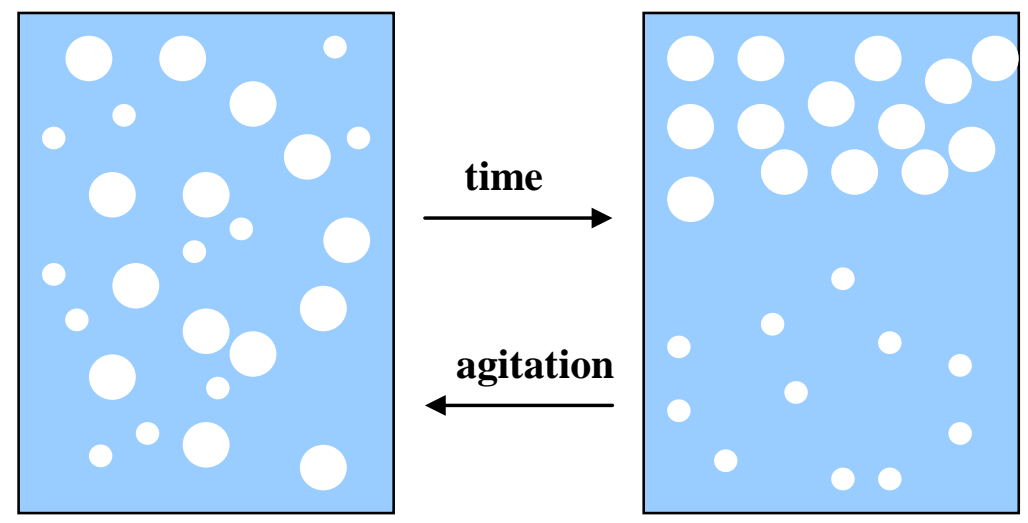

Figure 2.11. Creaming of an oil-in-water emulsion.

Creaming originates from the gravitational force when there is a difference of densities between the dispersed and the continuous phases. The creaming velocity $u$ of a spherical droplet is related to its radius $r$, the difference between the densities of the dispersed $\left(d_{1}\right)$ and continuous phases $\left(d_{2}\right)$, called $\Delta \rho=d_{2}-d_{1}$, the continuous phase viscosity $\eta_{2}$ and the dispersed phase viscosity $\eta_{1}$, by an equation suggested by Rybczynski and Hadamard [30, 31].

$$
u=\frac{2 g r^{2} \Delta \rho}{3 \eta_{2}} \times \frac{\eta_{1}+\eta_{2}}{3 \eta_{1}+2 \eta_{2}}
$$

The sign of $u$, and hence the direction in which the particle moves, depends on the relative values of the densities. In $\mathrm{O} / \mathrm{W}$ emulsions, oil density is usually smaller than water density and upward movement occurs and a concentration gradient builds up with the larger droplets staying at the top of the cream. The concentration of droplets $C(H)$ at height $H$ is given by Eq. 2.10 [1]. 


$$
C(H)=C_{0} e^{\frac{-4 / 3 \pi r^{3} \Delta \rho g H}{k T}}
$$

where $C_{0}$ is the concentration at $\mathrm{t}=0$ in the absence of a concentration gradient. In the case of small droplets $(<0.1 \mu \mathrm{m})$, creaming is prevented since Brownian motion exceeds the gravitational force

$\mathrm{kT}>>4 / 3 \pi \mathrm{r}^{3} \Delta \rho \mathrm{LL}$

where $L$ is the height of the container.

Inhibition of creaming is achieved by raising the viscosity of the continuous phase upon addition of a high-density material (polymer) and/or reducing the droplet size and/or adjusting the density of the dispersed phase to match closely the density of the external phase. Although creaming in general represents undesirable behaviour, there are instances where it is useful such as in the separation of cream from milk by centrifugation.

\subsubsection{Ostwald ripening}

Ostwald ripening is the observed increase of larger droplets at the expense of the smaller droplets, the latter being less thermodynamically stable due to a higher interfacial area (Figure 2.12).

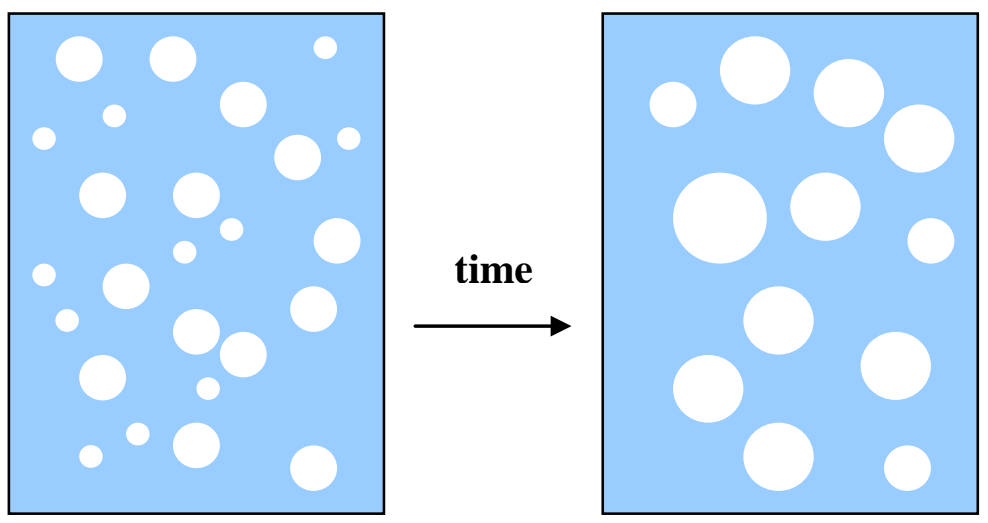

Figure 2.12. Ostwald ripening in an $\mathrm{O} / \mathrm{W}$ emulsion. 
The basis for the phenomenon is described by the Kelvin equation. Across a spherical interface of radius $r$ and interfacial tension $\gamma$, the pressure difference, as given by the Laplace equation, is

$$
\Delta P=\frac{2 \gamma}{r}
$$

The pressure gradient across a spherical interface decreases with size. Therefore, in an emulsion sample, the largest droplets grow at the expense of the smallest which evaporate preferentially. Because the system is in equilibrium (i.e. saturated with respect to vapour), the molecules vaporised from the small droplets simply add to the larger ones provided that the molecules of the dispersed phase have an appreciable diffusion coefficient in the continuous phase and through the interfacial film.

Kabalnov and co-workers [32-34] suggested that in an emulsion the rate of increase of the mean radius $\langle r\rangle$ or rate of Ostwald ripening is given by

$$
\frac{d\langle r\rangle^{3}}{d t}=\frac{8 \gamma D C V_{m}}{R T}
$$

where $D$ is the diffusion coefficient of the dispersed phase in the continuous phase, $C$ is the solubility of the dispersed phase in the continuous phase, $V_{m}$ is the molar volume of the dispersed phase and $\gamma$ is the interfacial tension.

One possible way to reduce Ostwald ripening is to incorporate a very small amount of a third component soluble in the dispersed phase but quite insoluble in the continuous phase [35] or produce a droplet size distribution as narrow as possible (similar pressure gradient at all interfaces) or increase the viscosity of the continuous phase.

\subsubsection{Flocculation}

Flocculation is the result of vdW attractive forces whereby flocs are formed, i.e. clusters of two or more droplets which behave kinetically as a unit, but in which the components of the cluster retain their identity. Individual droplets may join or 
leave the cluster at any time, and the number of units in the cluster may vary with time (Figure 2.13).

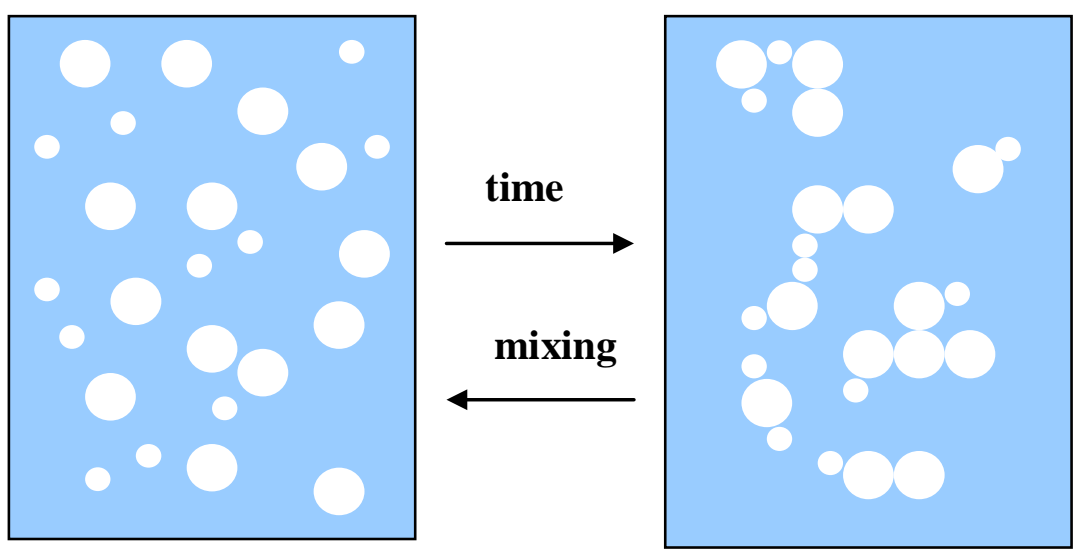

Figure 2.13. Flocculation in an $\mathrm{O} / \mathrm{W}$ emulsion.

The process of flocculation is associated with the presence of a secondary minimum in the energy of interaction-distance curve (Figure 2.8) of the colloidal system where attractive forces dominate. Flocculation is a reversible process, the lifetime of which depends on the depth of the secondary minimum. It is estimated that if the depth is less than $5 k T$ units, the flocculated particles can emerge from the minimum potential simply on the basis of their thermal kinetic energy or on gentle mixing [3].

Although addition of unadsorbed (free) long-chain surfactants or high molecular weight polymers in the continuous phase of an emulsion can impart stabilization by acting as a mechanical barrier between the droplets (depletion stabilization), it can also contribute to depletion flocculation (Figure 2.14). This occurs when two droplets approach closely enough so that the polymer molecules cannot fit into the region between the droplets, i.e. the interdroplet separation is smaller than the root-mean-square length of the polymer chain. The concentration of polymer in this region is depleted with respect to the bulk and polymers set up an osmotic force between the bulk continuous phase where they reside and the interdroplet space from which they are excluded [36]. 


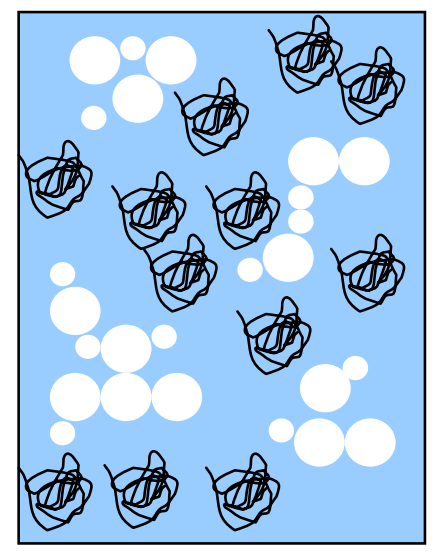

Figure 2.14. Depletion flocculation in an $\mathrm{O} / \mathrm{W}$ emulsion.

\subsubsection{Coalescence}

Coalescence is the irreversible fusion of two or more droplets to form a larger droplet (Figure 2.15).

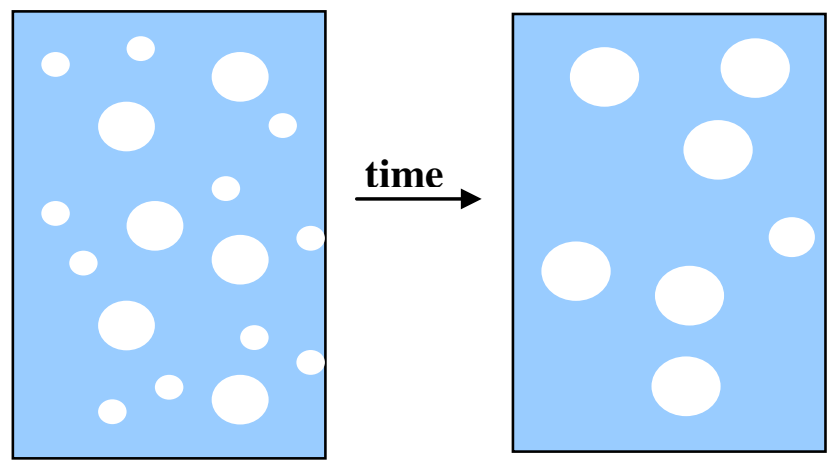

Figure 2.15. Coalescence in an $\mathrm{O} / \mathrm{W}$ emulsion.

It occurs when droplets have been close together for an extended period of time in a creamed layer, floc or during Brownian motion resulting in thinning and rupture of the liquid film of continuous phase separating the droplets [14].

When two droplets approach closely, their convex surfaces gradually become distorted from spherical symmetry and in the presence of surfactant a thin liquid lamella is formed between them as shown in Figure 2.16. 


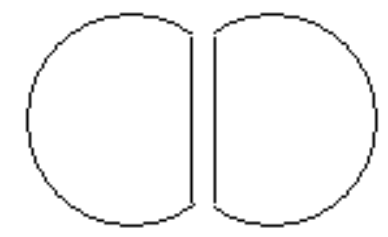

Figure 2.16. Lamella formation between droplets.

The lamella thickness depends on the nature of the colloidal forces between the droplet surfaces. Lamellae of the order of a few nanometres thick are called "black" films because they are thin enough for light waves reflected from front and back to be almost exactly out of phase and cancel (destructive interference). The colloidal forces manifest themselves in the disjoining pressure, which is the net force per unit area acting in the direction normal to the two flat parallel surfaces. In thermodynamics, the disjoining pressure $\Pi_{D}$ is defined as

$$
\Pi_{D}=-\left[\frac{\partial\left(G / A_{f}\right)}{\partial \tau}\right]_{T}
$$

where $G$ is the energy of formation of the thin film, $A_{f}$ the film area and $\tau$ the film thickness. The term $\Pi_{D}$ can be regarded as a sort of osmotic pressure difference between the liquid in the film and the bulk continuous phase. The drainage of liquid from the film, driven by the Laplace capillary pressure (Eq. 2.11), causes surfactant molecules to be swept along the two oil/water interfaces. This produces an interfacial tension gradient which acts to resist further tangential displacement of surfactant (Gibbs-Marangoni effect). However, for an emulsifier that is soluble in the droplets there is an infinite reservoir of surfactant available to replenish that swept away by drainage and so any contribution to stability from the GibbsMarangoni effect is minimal. Film rupture is a statistical process whose probability depends on the frequency of surface fluctuations and on the elastic properties of the emulsifier. Ripples on the surfaces of a plane-parallel thin film produce local fluctuations in film thickness (a critical thickness is required for film rupture). According to Vrij [14], the film is unstable with respect to rupture if the following inequality is satisfied 
$\frac{d^{2} G(h)}{d h^{2}}<-\frac{2 \pi^{2} \gamma}{A_{f}^{2}}$

where $\gamma$ is the interfacial tension and $G(h)$ is the energy of interaction between the droplets as a function of the surface-to-surface separation $h$.

Coalescence results in emulsion breakdown, i.e. the emulsion microstructure is lost and the system separates into the original individual phases. Coalescence is caused by the tendency for the system to minimize surface area while maximizing volume since a droplet formed by coalescence has a smaller surface area than that of its parent droplets together.

van den Tempel $[37,38]$ demonstrated that the total number of particles $N$ whether flocculated or not at time $t$ in a colloidal sample is given by

$$
N=\frac{N_{0}}{1+\beta N_{0} t}+\frac{\beta N_{0}{ }^{2} t}{\left(1+\beta N_{0} t\right)^{2}} \times\left(\frac{\beta N_{0}}{v}+\left(1-\frac{\beta N_{0}}{v}\right) \exp (-v t)\right)
$$

where $N_{0}$ is the initial number of dispersed droplets, $\beta$ is the flocculation rate constant and $v$ is the rate of coalescence.

Continuation of coalescence makes droplets subject to significant creaming because the mean radius of the distribution increases. The viscosity of the continuous phase plays an important role with respect to coalescence. Concentrated emulsions are often more stable than diluted ones since the viscosity of the continuous phase rises with the number of droplets and the number of collisions is reduced. 


\section{Chapter 3 Toluene/Triton X-100/water emulsions}

\subsection{Choice of the emulsifier}

For the study of emulsion microstructure and dynamics, the ternary toluene/Triton X-100/water system was investigated. Triton X-100 is a nonionic surfactant. Its chemical formula is shown in Figure 3.1.

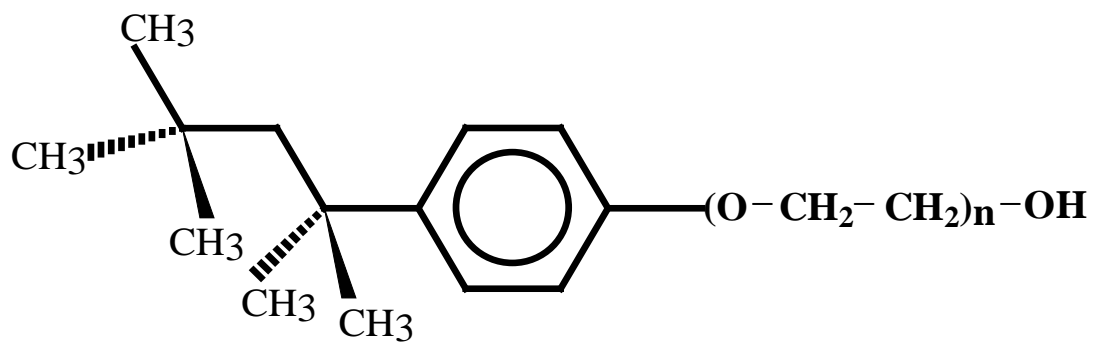

Figure 3.1. Chemical formula of Triton X-100; $n=10$ on average.

The hydrophobic tail of this surfactant is composed of the 4-(1,1,3,3,tetramethylbutyl) phenoxy group and the hydrophilic head consists of an ethylene oxide oligomer with an average of ten blocks per molecule (actually contains a narrow spectrum of block units, with a distribution peaking at the value $n=10$ ). Triton X-100 is synthesized by the polymerization reaction between $(1,1,3,3,-$ tetramethylbutyl) phenol and ethylene oxide. The ethylene oxide groups may be added to any desired extent to alter the solubility and the stabilizing properties of the surfactant [3]. As a nonionic surfactant, Triton X-100 is part of the largest growing group of emulsifying agents because they do not depend on water hardness and $\mathrm{pH}$. Moreover, the effectiveness of the hydrophilic portion of the surfactant molecule can be modified to stabilize an emulsion with a specific composition. The hydrophilic-lipophilic balance (HLB) of Triton X-100 is 13.5 [39] making this surface-active agent an effective emulsifier to stabilize $\mathrm{O} / \mathrm{W}$ emulsions. 
In addition to effectiveness, other considerations in choosing Triton X-100 are cost, commercial availability and minimization of the types of repulsive forces involved, i.e. electrostatic repulsion is negligible compared to steric repulsion.

\subsection{Phase diagram}

In Figure 3.2 is shown the phase diagram of the ternary toluene/Triton $\mathrm{X}$ 100/water system as determined by Liu and coworkers [40]. The straight line indicates the region where kinetically stable emulsions can be formed, i.e. macroscopic phase separation occurs for times longer than $\sim 30 \mathrm{~min}$. For emulsions to be realized in this system, toluene concentration must be comprised between 5 and $70 \mathrm{wt} \%$ and a constant toluene-to-surfactant weight ratio of $5: 1$ is required. Deviation from this ratio results in immediate macroscopic phase separation indicating that the intermolecular interactions between Triton X-100 and toluene dominate the ternary system by controlling the characteristics of the interfacial domain such as the interfacial tension, the Gibbs elasticity and the curvature of the interface. If the oil-to-surfactant weight ratio is not maintained to $5: 1$, these three controlling factors are not mutually satisfied and no emulsion can be stabilized.

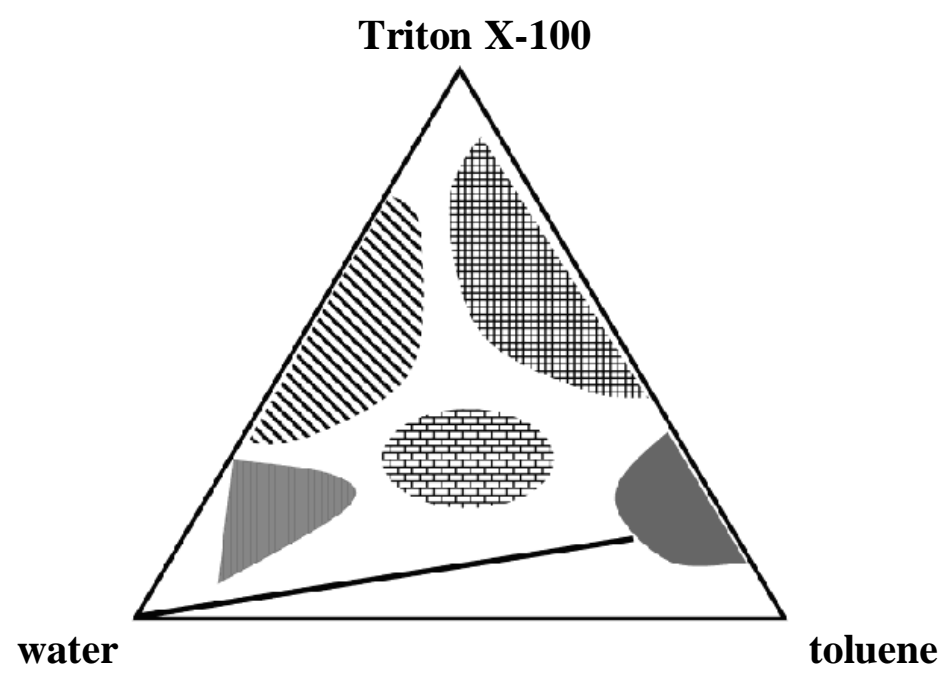

Figure 3.2. Phase diagram of the toluene/Triton $X-100 /$ water system taken from reference [40]. Microemulsions are formed in the hashed region, liquid crystals in the stripes, twophase Winsor emulsions in the solid zone, and extremely temperature sensitive two- and three-phase Winsor emulsions in the bricked area. The straight line indicates the region

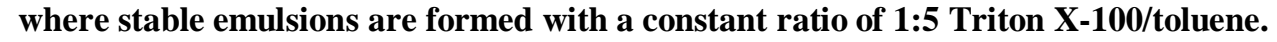




\subsection{Emulsification}

The three emulsion constituents including Triton X-100, toluene and water are weighed separately to prepare a final total emulsion mass of $15 \mathrm{~g}$. The oil-tosurfactant weight ratio is maintained to 5:1. Triton X-100 was purchased from BDH with a purity of $98 \%$ and a single batch number was used for all experiments. Toluene with a purity $>99.9 \%$ was purchased from Sigma-Aldrich and water was of Milli-Q grade. All the experiments are performed at $298 \mathrm{~K}$. Before emulsification, Triton X-100 is predissolved in toluene since it is more soluble in aromatic oils than in water and the mixture; oil plus surfactant is added to water (agent-in-oil method). Energy input is achieved by a blade stirrer from Warring (Figure 3.3) at 22,000 rpm for $1 \mathrm{~min}$ delivering an energy of $65 \mathrm{~J} / \mathrm{g}$ of emulsion [41].

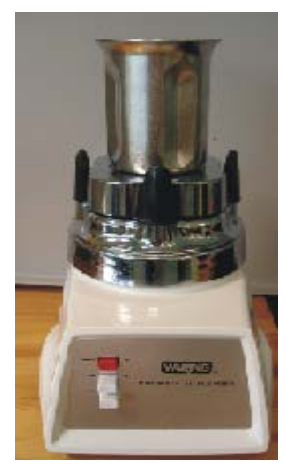

Figure 3.3. Blender from Warring used for emulsification.

Oil-in-water $(\mathrm{O} / \mathrm{W})$ emulsions are exclusively formed on a toluene continuum between 5 and $70 \mathrm{wt} \%$ corresponding to volume fractions of the dispersed phase between 5.8 and $83.5 \%$. The determination of the type of emulsion, i.e. O/W independent of oil concentration was achieved by both adding a water soluble dye (an emulsion mixes immediately with any liquid that is miscible with its continuous phase) and measuring the diffusion coefficient of the continuous phase using PFG-NMR [40]. 


\subsection{Experimental techniques}

\subsubsection{Static Light Scattering}

\subsubsection{Introduction}

Light can be described as simultaneous oscillating electric and magnetic fields perpendicular to each other. The way light propagates through matter is determined by the dielectric properties of that matter. The process of light scattering arises from a complex interaction between an incident electromagnetic wave and the electron cloud of a particle [42]. The electron movements within the particle's constituent molecules are perturbed periodically with the same frequency as the electric field of the incident wave. The oscillation or perturbation of the electron cloud results in a periodic separation of charge within the molecule, which is called an induced dipole moment. The oscillating induced dipole moment is manifest as a source of electromagnetic radiation, thereby resulting in scattered light. In colloidal systems, the scattering of light by particles including droplets varies as a function of both the size and the shape of the particles and the differences between the dielectric and magnetic properties of the particles and the medium. However, the difference between the magnetic properties of the two phases is usually negligible. The dielectric properties of a material are related to its refractive index. In general, the refractive index $m$ of a material is represented by the complex notation defined in Eq. 3.1.

$$
m=n-i \theta
$$

where $n$ equals the speed of light in vacuum divided by the speed of light in the material (refraction of light) and is commonly measured with a refractometer, while the imaginary part $\theta$ is associated to the absorption of light. The absorption coefficient $\alpha$ of a material is related to $\theta$ via Eq. 3.2.

$$
\alpha=\frac{4 \pi \theta}{\lambda}
$$


Although the value of $\theta$ is never exactly zero, materials with a value approaching zero are termed dielectrics.

The dependence of scattering on particle shape is complex which is why very often it is assumed that the scattering particles are spheres, hence the mathematics of light scattering can be simplified to three size ranges set by the ratio of the wavelength of the incident light to the size of the particle [43]. The Mie theory (after Gustav Mie) completely solves the equations for interaction of light with matter and consists of the general spherical scattering solution for absorbing and non-absorbing materials without a particular bound on particle size. For particles small compared to the wavelength of light, the Mie theory simplifies to the Rayleigh theory whereas for particles larger than the wavelength of light, it simplifies to the Fraunhofer theory or Fraunhofer diffraction.

\subsubsection{Particle sizing}

Measuring droplet size using static light scattering (SLS) relies on the fact that particles passing through a laser beam scatter light at an angle that is inversely proportional to the particle size. Scattering intensity is also dependent on particle size, increasing with diameter.

In a typical droplet sizing instrument (Figure 3.4), a laser provides a source of coherent intense light of fixed wavelength; He-Ne gas lasers $(\lambda=633 \mathrm{~nm})$ are the most common as they offer the best stability (especially with respect to temperature) and a high signal-to-noise ratio. Smaller wavelengths (blue light sources) are also used to improve the sensitivity to sub-micron particles. A sample unit ensures that the material under test passes through the laser beam as a homogeneous stream of particles in a known, reproducible state of dispersion. A series of detectors finally measure the light intensity pattern produced over a wide range of angles (Figure 3.5). 


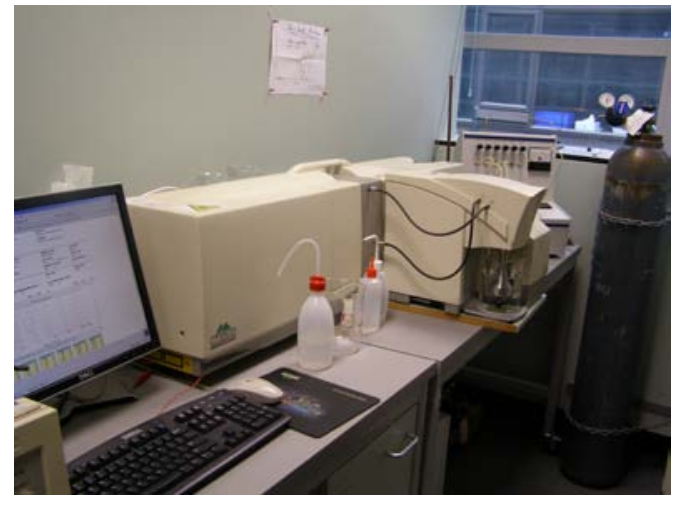

Figure 3.4. Mastersizer 2000 from Malvern Instruments.

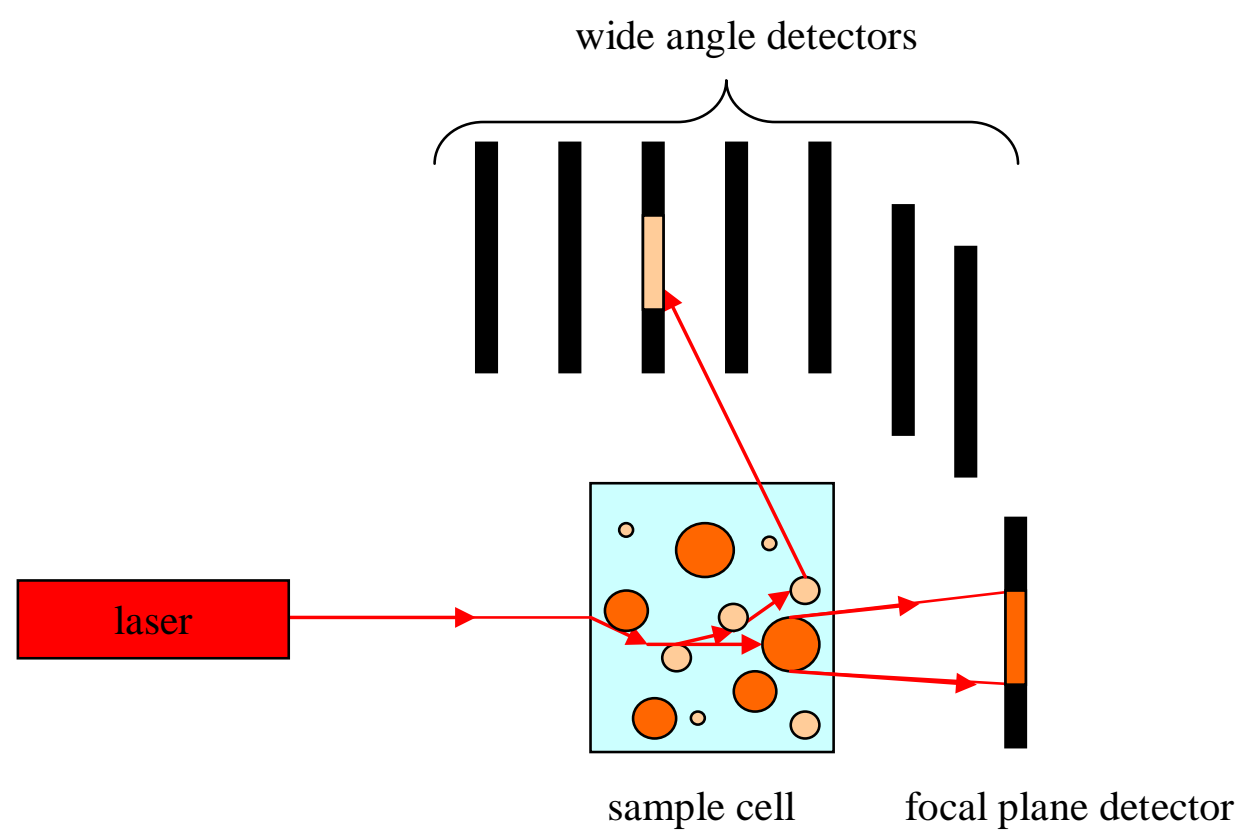

Figure 3.5. Principle of particle sizing by SLS; the light source emitted by a laser is scattered by the particles with an intensity and at an angle which are size dependent. The focal plane detector is sensitive to scattering by large particles whereas wide angle detectors are sensitive to the light scattered by small particles.

\subsubsection{Data analysis}

For the study of our emulsion samples, droplet size distributions were measured using a Mastersizer 2000 from Malvern Instruments (Figure 3.4) with wavelengths of 633 and $452 \mathrm{~nm}$, covering a size range from 0.02 to $2000 \mu \mathrm{m}$. A typical droplet size distribution of our emulsion samples is shown in Figure 3.6. 


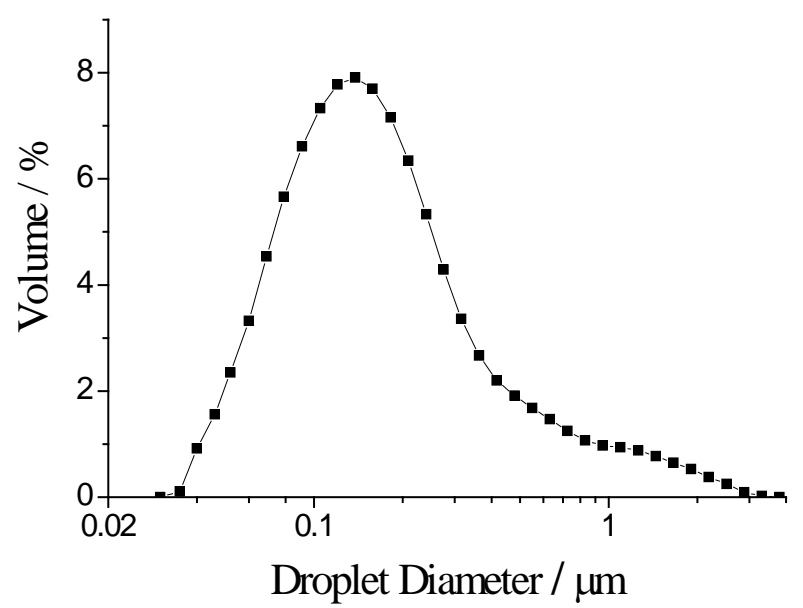

Figure 3.6. Droplet size distribution of a toluene/Triton X-100/water emulsion prepared with 20 wt \% toluene.

Droplet size distributions are determined by comparing experimental scattering patterns (intensity versus scattering angle) with theoretical scattering patterns using Mie theory [44].

Indeed, the position of the maxima in the intensity of the scattered light as a function of scattering angle depends on the particle size. Mie theory gives the complete analytical solution of Maxwell's equations for the scattering of electromagnetic radiation by spherical particles [45]. The theory predicts the intensity of scattered light which is proportional to the differential cross section defined as the probability to observe scattered light per solid angle unit, such as within a given cone of observation, if the target is irradiated by a flux of one particle per surface unit. The total scattering cross section is the sum of the differential cross sections over the whole sphere of observation and is expressed as the infinite series

$$
\sigma_{\text {Miescatt }}=\frac{\lambda_{0}^{2}}{2 \pi n_{\text {med }}{ }^{2}} \sum_{n=1}^{\infty}(2 n+1)\left(\left|a_{n}\right|^{2}+\left|b_{n}\right|^{2}\right)
$$

where $\lambda_{0}$ is the wavelength of the incident beam in vacuum, $n_{\text {med }}$ is the refractive index of the dispersant and the coefficients $a_{n}$ and $b_{n}$ are given by

$$
a_{n}=\frac{\mu m^{2} j_{n}(m x)\left[x j_{n}(x)\right]^{\prime}-\mu_{1} j_{n}(x)\left[m x j_{n}(m x)\right]^{\prime}}{\mu m^{2} j_{n}(m x)\left[x h_{n}^{(1)}(x)\right]^{\prime}-\mu_{1} h_{n}^{(1)}(x)\left[m x j_{n}(m x)\right]^{\prime}}
$$




$$
b_{n}=\frac{\mu_{1} j_{n}(m x)\left[x j_{n}(x)\right]^{\prime}-\mu j_{n}(x)\left[m x j_{n}(m x)\right]^{\prime}}{\mu_{1} j_{n}(m x)\left[x h_{n}^{(1)}(x)\right]^{\prime}-\mu h_{n}^{(1)}(x)\left[m x j_{n}(m x)\right]^{\prime}}
$$

where the $j_{n}$ s are spherical Bessel functions of the first kind, the $h_{n}$ s are spherical Hankel functions, and $\mu_{1}$ and $\mu$ are the magnetic permeability of the sphere and surrounding medium, respectively. Primes indicate derivatives with respect to the size parameter $x$ given by

$x=\frac{2 \pi n_{m e d} a}{\lambda_{0}}$

where $a$ is the particle radius.

The shape of emulsion droplets is expected to fluctuate under the high shear conditions of the SLS measuring cell. Hence droplets with irregular shape instead of spherical shape must be considered. These irregularly-shaped droplets produce optical noise by causing uneven polarisation of the scattered light, especially at wide scattering angles. As a consequence the "irregular shape" function for droplet sizing in emulsion systems must be activated. This function consists in rejecting the optical noise caused by interface fluctuations by decreasing the weighting of the raw data at wide scattering angles before applying Mie theory, the latter being valid for spherical particles only. The rejection of optical noise caused by irregularly-shaped droplets leads to a better fitting of the raw data using Mie theory.

\subsubsection{Dynamic Light Scattering}

\subsubsection{Introduction}

Dynamic light scattering (DLS) also known as photon correlation spectroscopy determines the size of particles including droplets in the nanometre range by measuring Brownian motion, i.e. the movement of particles due to the random collisions with the molecules of the liquid that surrounds them. Particles are illuminated by a light source and light is scattered in all directions giving a 
speckle pattern on a detector held close to the particles. The speckle pattern consists of bright areas where the light waves interfere constructively (similar phases) and dark areas where no light is detected (the phases are mutually destructive and cancel each other out). As the particles are constantly in motion, the intensity of the bright and dark areas fluctuates over time. The DLS instrument measures the rate of the intensity fluctuations and relates this to the particle size [46].

\subsubsection{Instrument}

For the determination of the size of the smallest droplets in our emulsion samples, a Zetasizer nano-ZS from Malvern Instruments operating with a $633 \mathrm{~nm}$ laser and covering a size range from $0.6 \mathrm{~nm}$ to $6 \mu \mathrm{m}$ was used. The instrument comprises six main components (Figure 3.7). First the laser provides a coherent light source to illuminate the sample particles within a cell. Most of the laser beam passes straight through the sample, but some is scattered by the particles and a detector measures the intensity of the scattered light. An attenuator placed immediately in front of the light source adjusts the laser intensity and therefore the intensity of the scattering. Indeed, for samples that do not scatter much light, such as very small particles or samples of low concentration, the intensity of the scattered light needs to be increased. On the contrary, for samples that scatter a large amount of light, such as large particles or samples of higher concentration, the intensity of the scattered light must be decreased to avoid saturation of the detector. Also, a moveable lens located between the cell and the detector allows the focus position within the cell to be changed in order to increase the detector sensitivity. Small particles scattering light with a low intensity are detected away from the cell wall to maximize the signal by reducing the scattering signal from the cell wall whereas large particles are preferentially detected closer to the cell wall to reduce the effect of multiple scattering (the scattering of the cell wall is negligible in this case). The detector is placed at an angle of $173^{\circ}$ relative to the direction of the transmitted light, i.e. the instrument functions in backscattered mode. The main advantage of the backscattered detection is that the incident beam does not have to travel through the entire sample. Therefore, multiple scattering (the scattered 
light from one particle is itself scattered by other particles) is minimised as well as the scattering of large dust particles since large particles mainly scatter in the forward direction. However, the use of a unique detector to collect the intensity of the scattered light limits the range of scattering angles and particles larger than 6 $\mu \mathrm{m}$ (high limit of detection) cannot be probed.

Finally the scattering intensity signal passes from the detector to a digital signal processor called a correlator so as to compare the scattering intensity at successive time intervals before sending the information to a computer where the size distribution is delivered.

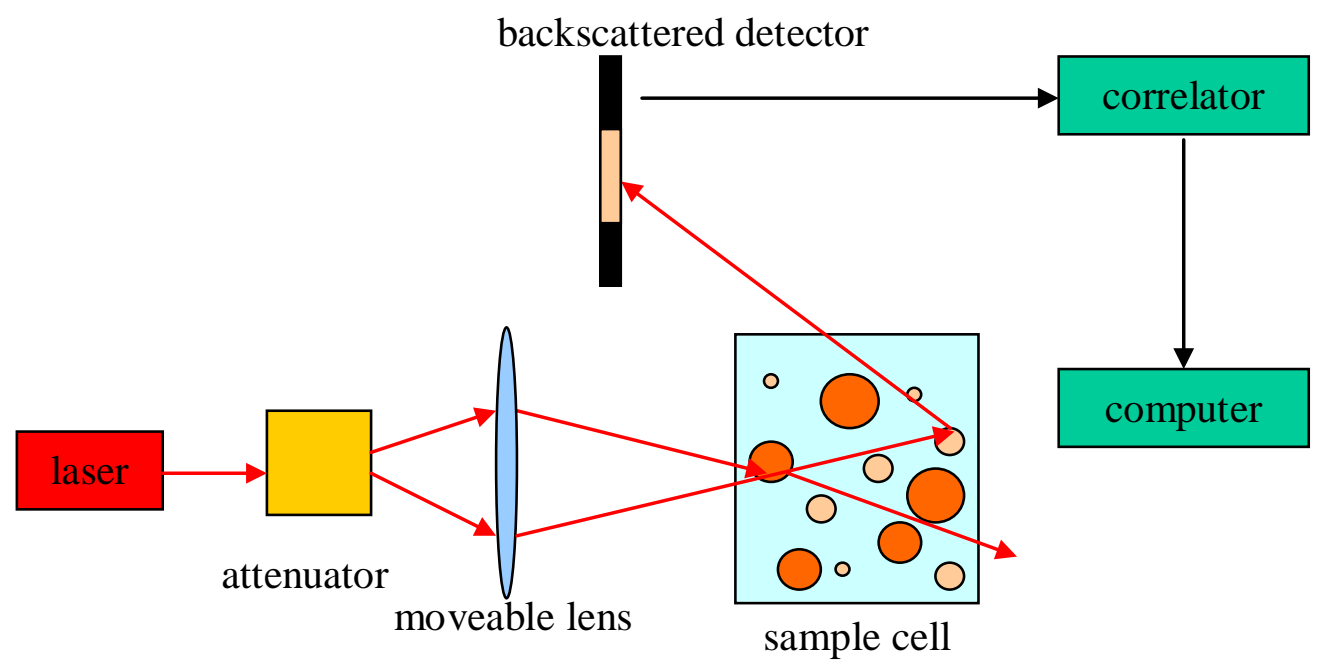

Figure 3.7. A typical DLS instrument; the light emitted by a laser source is scattered by particles and detected at wide angles by a backscattered detector at successive time intervals.

\subsubsection{Data analysis}

The droplet size distribution is determined by measuring the rate of the intensity fluctuation of the scattered light [47]. The correlator measures the degree of similarity between the signals of a particular part of the speckle pattern over a period of time. At $t=0$, comparing the scattering signal with itself gives a perfect correlation which is reported as 1 . Over time, any two consecutive signals become progressively less similar as the particles move in random directions due to Brownian motion. The correlation between two consecutive signals therefore reduces with time until reaching zero after a few microseconds. Since the rate of 
motion of the particles decreases with particle size, the rate of intensity fluctuation or decay of the correlation function also decreases with particle size (Figure 3.8).

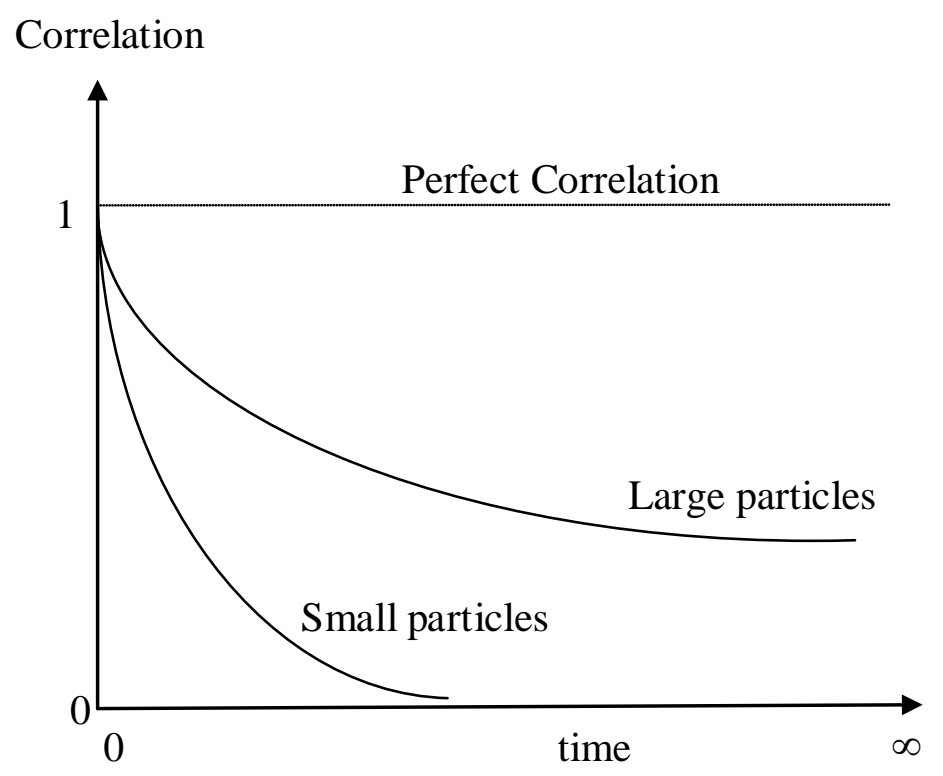

Figure 3.8. Correlation function for different particle sizes.

The instrument software extracts the different decay rates of the correlation function and generates a size distribution (an intensity distribution is produced which can be converted to a volume distribution). A typical size distribution is illustrated in Figure 3.9, here for the oil swollen micelles present in the continuous phase of a toluene/Triton X-100/water emulsion.

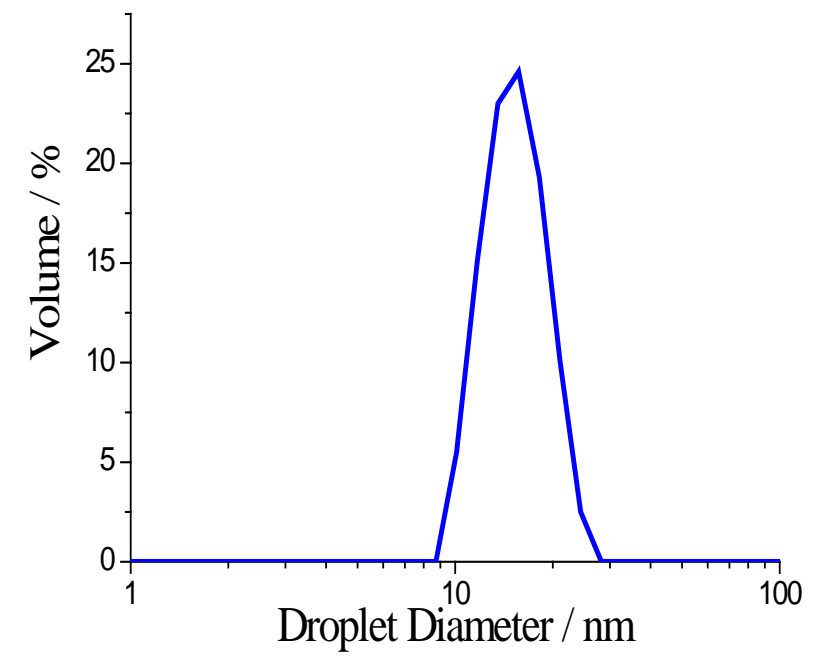

Figure 3.9. Size distribution of the oil swollen micelles present in a toluene/Triton $\mathrm{X}-100 /$ water emulsion prepared with $15 \mathrm{wt} \%$ toluene. 


\subsubsection{Pulsed field gradient nuclear magnetic resonance}

\subsubsection{Introduction}

Because of its noninvasive nature, pulsed field gradient nuclear magnetic resonance (PFG-NMR) spectroscopy is a unique tool for studying the dynamics and microstructure of colloidal systems such as emulsions. It is a rapid and convenient means for measuring translational motion compared to the radioactive tracer techniques. The method is based on the attenuation of an echo signal to measure the displacement of the spins. Information can be obtained about the microstructure $[40,48]$, the dynamics $[41,49]$ or the droplet size distribution [5052] of colloidal samples.

Self-diffusion is the random translational motion of molecules or particles due to thermal energy. Translational diffusion is the most fundamental form of transport and is responsible for all chemical reactions. Diffusion is also closely related to molecular size, as can be seen from the Stokes-Einstein equation (Eq. 3.4).

$$
D=\frac{k T}{6 \pi \eta r}
$$

where $D$ is the self-diffusion coefficient, $k$ is the Boltzmann constant, $T$ is the absolute temperature, $\eta$ is the viscosity of the suspending medium and $r$ is the hydrodynamic radius.

\subsubsection{The pulsed gradient spin echo}

The pulsed gradient spin echo (PGSE) is a non-invasive technique developed by Stejskal and Tanner [53]. It is a modification of the Hahn spin echo experiment [54] which enables diffusion measurements of molecules and particles in fluid samples. This method is particularly suitable for studying the dynamics and microstructure of colloidal systems such as emulsions. 

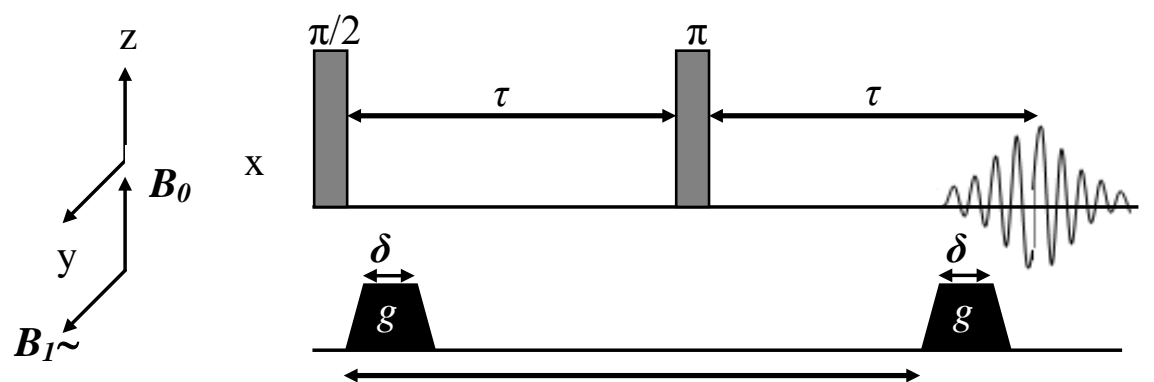

(a)

$\Delta$

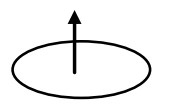

$\downarrow \pi / 2 \mathbf{R F}$

(b)
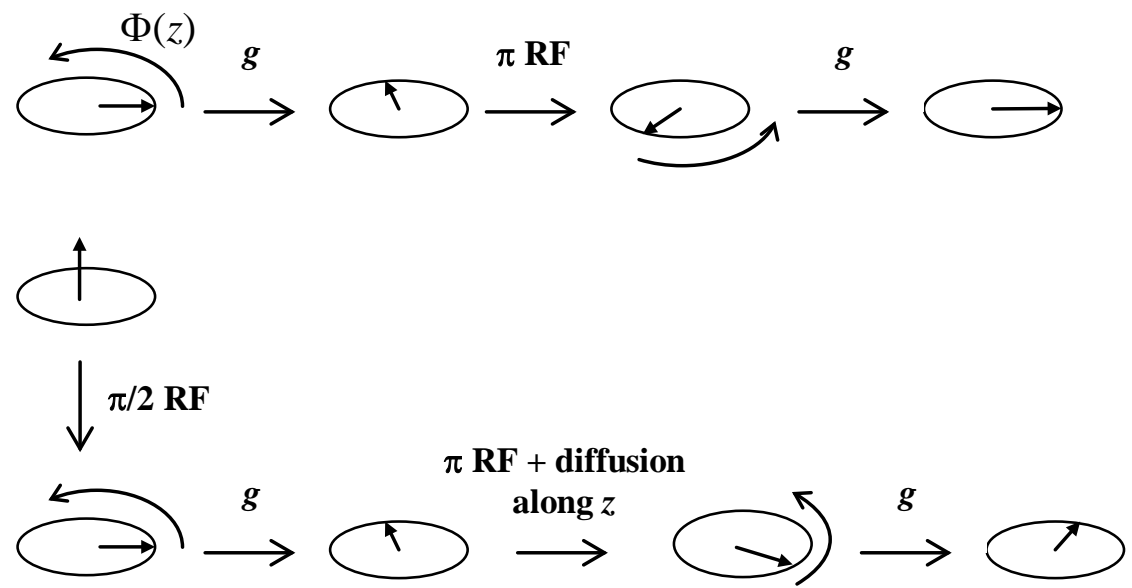

(c)

Figure 3.10. PGSE pulse sequence (a); position of the spins during the PGSE in the absence (b) or presence (c) of diffusion during the observation time, $\Delta$.

In a pulsed gradient spin echo experiment (Figure 3.10a), a constant magnetic field $B_{0}$ is generated along the $z$ direction by a superconducting magnet. The ${ }^{1} \mathrm{H}$ nuclear spins of a sample interact with the applied magnetic field $B_{0}$ and rotate about the direction of the field with an angular frequency $\omega_{0}$ called the Larmor frequency according to Eq. 3.5.

$$
\omega_{0}=-\gamma B_{0}
$$

where $\gamma$ is the hydrogen spin gyromagnetic ratio $\left(\gamma=2.67 \times 10^{8} \mathrm{rad} \mathrm{s}^{-1} \mathrm{~T}^{-1}\right)$.

The net magnetization of the nuclear spins is parallel to $B_{0}$. $A \pi / 2$ radio frequency (RF) pulse feeds a coil surrounding the sample and a magnetic field $B_{1}$ is created in the xy transverse plane during a very short period of time $(\sim 7 \mu \mathrm{s})$ oscillating 
with an angular frequency $\omega_{1}$ in order to tilt the net magnetization through $90^{\circ}$. The nuclear spins precess in the transverse xy plane with an angular frequency $\Omega$ in the rotating frame given by Eq. 3.6 (the rotating frame rotates about the $\mathrm{z}$ axis with an angular frequency $\left.-\omega_{1}\right)$.

$$
\begin{aligned}
\Omega & =\omega_{0}-\omega_{\text {rotatingframe }} \\
& =\omega_{0}+\omega_{1}
\end{aligned}
$$

A pulsed magnetic field gradient of amplitude $g$ and duration $\delta$ is applied to spatially label the $z$ position of the spins in the sample by imposing a $z$-dependent phase angle $\Phi(z)$ given by Eq. 3.7.

$$
\begin{aligned}
\Phi(z) & =\Omega(z) \delta \\
& =-\gamma B(z) \delta \\
& =-\gamma\left(B_{0}+g z\right) \delta
\end{aligned}
$$

The effect of a $\pi$ RF pulse is to move the nuclear spins to a mirror image position and cause the reversal of the sign of the phase angle as depicted in Figure 3.10b. A second magnetic field gradient identical to the first one attributes to the spins a phase angle equal to the one acquired after the first gradient (Eq. 3.7). If the spins do not diffuse during the observation time, $\Delta$, the effects of the two gradients cancel out, all the spins refocus and a maximum echo signal is obtained (Figure $3.10 \mathrm{~b})$. In the presence of diffusion along the $z$ axis (Figure 3.10c), the phase angle of the spins measured after the first gradient is different in magnitude from the phase angle of the spins after the second gradient since each spin is located in a different $z$ position and therefore in a different magnetic field. Hence, the spins precess with altered angular frequencies and the echo signal (transverse magnetization) measured on the $\mathrm{x}$ axis is smaller. Stejskal and Tanner have shown that the echo signal is attenuated according to Eq. 3.8.

$$
I_{(g, \delta, \Delta)}=\sum_{i} I_{0, i} \exp \left(\frac{-2 \tau}{T_{2}}\right) \exp \left(-D_{i} \gamma^{2} g^{2} \delta^{2}\left(\Delta-\frac{\delta}{3}\right)\right)
$$


where $I_{0, i}$ is the contribution of the particle $i$ to the signal intensity that would be observed immediately after the first $90^{\circ}$ radio frequency pulse, $D_{i}$ is the diffusion coefficient of the particle $i$ and $T_{2}$ is the spin-spin relaxation constant of the substance. The exponential $T_{2}$ term reflects the decay of the transverse magnetization to zero due to the generation of local magnetic fields destroying the coherence of the spins in the transverse plane.

If the duration $\tau$ is kept constant during the experiment, it is then possible to separate out the transverse relaxation and the diffusion contributions and the equation is simplified as described in Eq. 3.9.

$$
I_{(g, \delta, \Delta)}=\sum_{i} I_{0,2 \tau} \exp \left(-D_{i} \gamma^{2} g^{2} \delta^{2}\left(\Delta-\frac{\delta}{3}\right)\right)
$$

where $I_{0,2 \tau}$ is the signal intensity at $2 \tau$ in the absence of a gradient.

In the case of a pure substance or a monodisperse sample the echo attenuation can be described by a single diffusion coefficient, that is the summation in Eq. 3.9 reduces to a single term (Eq. 3.10$)$.

$$
I_{(g, \delta, \Delta)}=I_{0,2 \tau} \exp \left(-D \gamma^{2} g^{2} \delta^{2}\left(\Delta-\frac{\delta}{3}\right)\right)
$$

\subsubsection{The pulsed gradient stimulated echo}

The pulse sequence for the pulsed gradient stimulated echo (PGSTE) technique is sown schematically in

Figure 3.11. 


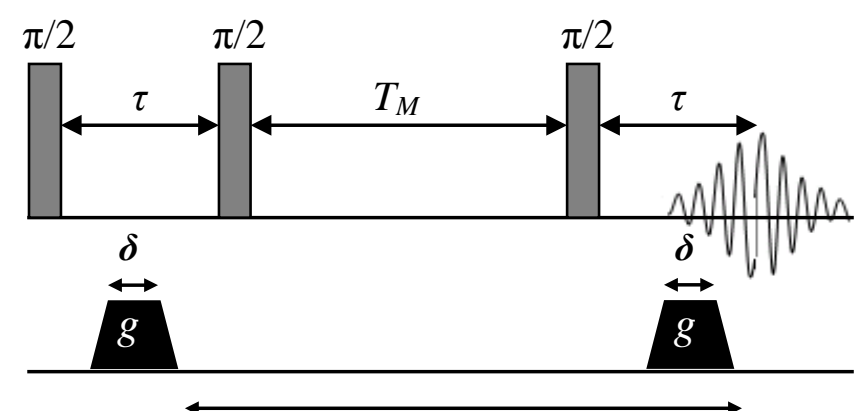

$\Delta$

Figure 3.11. Pulsed gradient stimulated echo pulse sequence. Three radio frequency pulses rotate the net magnetization to a $\pi / 2$ angle. A first gradient of amplitude $g$ and duration $\delta$ labels the positions of the spins whereas a second gradient, after an observation time, $\Delta$, recovers the signal to form an echo.

The main difference with a pulsed gradient spin echo is the replacement of the $\pi$ RF pulse by two $\pi / 2$ RF pulses. During the time $T_{M}$, there is no longer a transverse magnetization in the xy plane decaying with a time constant $T_{2}$ but rather a longitudinal magnetization decaying with a time constant $T_{1}$ called the spin-lattice relaxation constant. The $T_{1}$ constant reflects the return of the net magnetization to its equilibrium position that is along the $z$ axis.

The PGSTE experiment is suitable for systems with very short $T_{2}$ constants $(\sim<1$ s) where the echo signal decays to zero very rapidly. The method allows longer observation times, $\Delta$, to be probed by taking into account the $T_{1}$ relaxation in addition of the $T_{2}$ relaxation because in many systems, $T_{1}$ is significantly longer than $T_{2}$ (note that for pure water, $T_{1}=T_{2} \sim 3 \mathrm{~s}$ ). The echo attenuation of a PGSTE pulse sequence is given by Eq. 3.11[55].

$$
I_{(g, \delta, \Delta)}=\sum_{i} \frac{I_{0, i}}{2} \exp \left(\left(\frac{-2 \tau}{T_{2}}\right)-\left(\frac{T_{M}}{T_{1}}\right)\right) \exp \left(-D_{i} \gamma^{2} g^{2} \delta^{2}\left(\Delta-\frac{\delta}{3}\right)\right)
$$

If the durations $\tau$ and $T_{M}$ are kept constant during the experiment, again it is possible to separate out the transverse and longitudinal relaxation constants from the diffusion contributions and the equation simplifies to

$$
I_{(g, \delta, \Delta)}=\sum_{i} I_{0, T_{M}+2 \tau} \exp \left(-D_{i} \gamma^{2} g^{2} \delta^{2}\left(\Delta-\frac{\delta}{3}\right)\right)
$$


where $I_{0, T M+2 \tau}$ is the signal intensity at $2 \tau+T_{M}$ in the absence of a gradient.

In the case of a pure substance or a monodisperse sample, the summation in Eq. 3.12 reduces to a single term and the echo attenuation can be described by a single diffusion coefficient (Eq. 3.13)

$$
I_{(g, \delta, \Delta)}=I_{0, T_{M}+2 \tau} \exp \left(-D \gamma^{2} g^{2} \delta^{2}\left(\Delta-\frac{\delta}{3}\right)\right)
$$

\subsubsection{Experimental equipment}

All PFG-NMR experiments were carried out using a Bruker Avance-460 spectrometer (Figure 3.12).

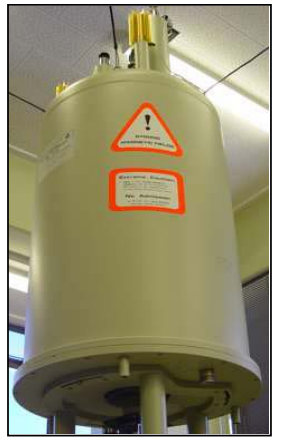

Figure 3.12. Bruker Avance-460 spectrometer.

To perform a PFG-NMR experiment, the spectrometer requires the following components as illustrated in Figure 3.13.

A superconducting magnet capable of generating an intense and homogeneous magnetic field $\mathrm{B}_{0}$. It consists of a coil of wire through which a current passes. The superconducting state (i.e. the resistance is zero) is achieved by immersing the coil in a bath of liquid helium and the magnet is insulated with a surrounding bath of liquid nitrogen under vacuum conditions.

$\checkmark$ Several shim coils surrounding the sample in order to produce a magnetic field that is as homogeneous (i.e. no variation over space) as possible, hence achieving a maximum resolution of the peaks. The shim coils produce tiny magnetic fields with a particular spatial profile which cancel 
out the small residual inhomogeneities in the main magnetic field. The current through each of the shim coils is manually adjusted by the operator until maximum peak sharpness is obtained.

$\checkmark$ A probe consisting of a cylindrical metal tube in which the coil used to both excite and detect the NMR signal is located as well as a heater and a cooling airflow to maintain the temperature of the sample within $0.1^{\circ} \mathrm{C}$. For that purpose, the probe needs to be placed as close as possible to the sample and is placed into the shaft of the magnet so that the sample can come down from the top of the shaft into the probe.

$\checkmark$ A high power RF transmitter capable of delivering short pulses.

$\checkmark$ A receiver consisting of amplifiers to detect and amplify the free induction decay.

$\checkmark$ A computer to control and process the data.

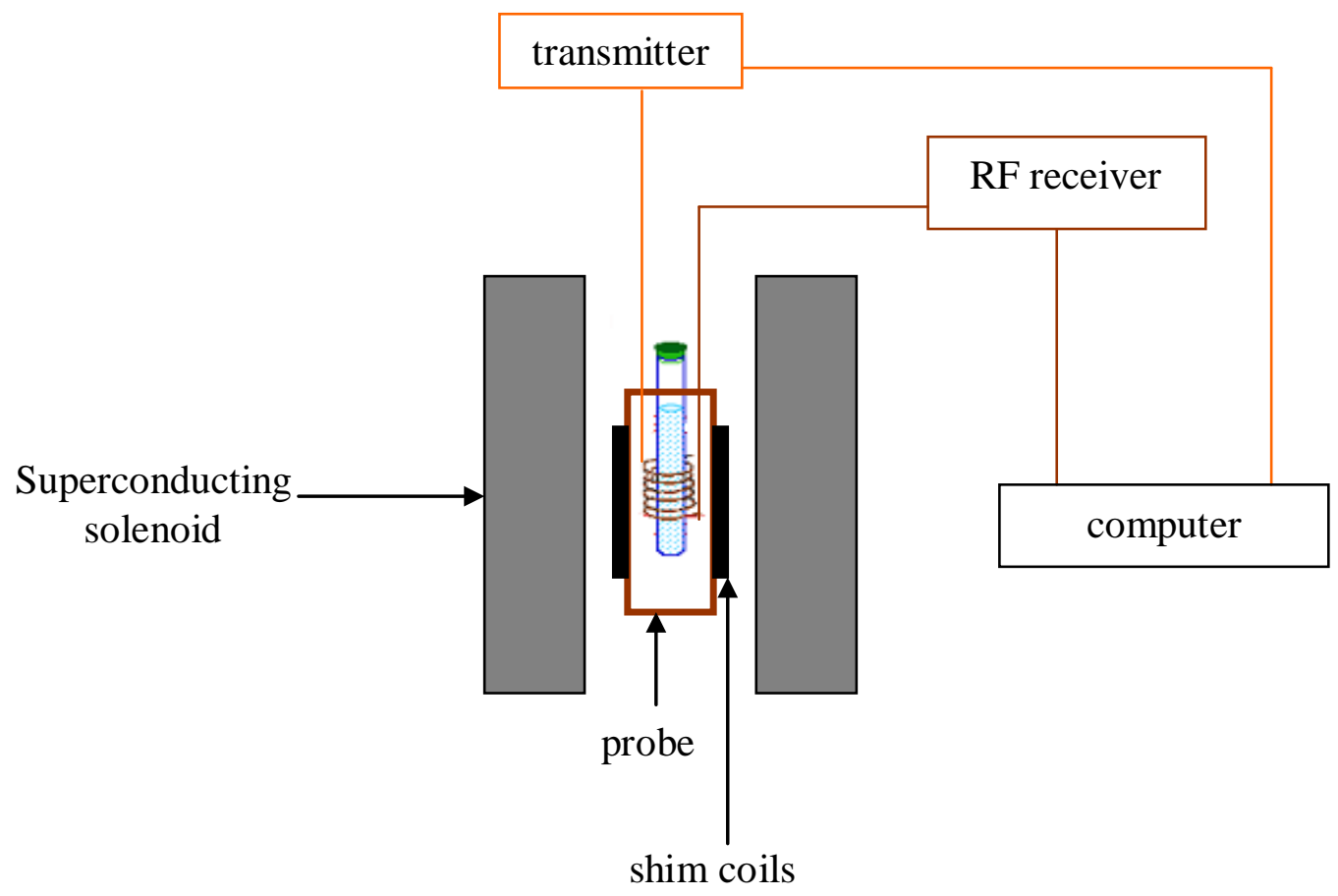

Figure 3.13. Schematic of the key components of a spectrometer. A superconducting solenoid produces an intense magnetic field and shim coils optimise the field homogeneity. The sample is inserted into a probe which produces $\pi / 2$ radio frequency pulses (excitation) and detect the free induction decay (signal). 


\subsubsection{Data analysis}

The PFG-NMR technique has several advantages for the study of emulsions. It requires no sample manipulation (which means that kinetically stabilized systems such as emulsions are observed under no external influence), only a small sample volume is required and diffusion coefficients as low as $\sim 1.0 \times 10^{-14} \mathrm{~m}^{2} \mathrm{~s}^{-1}$ can be measured [56]. The technique provides information on the interactions within the system and the structure of the diffusing molecules and droplets. Ideally, the interaction between the droplets and the overall structure of the complex fluid is reflected in the values of the diffusion coefficients. In general, PFG-NMR has been applied to the detection of the diffusion of individual molecules moving within a solvent, where there is no boundary to diffusion, so-called unrestricted or free diffusion. In an isotropic system, without thermal or concentration gradients, the limiting mean square displacement of a molecule or droplet during a time $\Delta$ in one dimension is calculated via the Einstein relation (Eq. 3.14).

$$
\left\langle Z^{2}\right\rangle=2 D \Delta
$$

where the angular brackets symbolize a time average.

When unrestricted diffusion occurs, the diffusion coefficient is independent of the observation time, $\Delta$. By contrast, in emulsions the movement of molecules inside a droplet, or of droplets themselves can be restricted by the size and the shape of the droplets. Such restrictions depend on the magnitude of the diffusive length scale associated with the time $\Delta$, in comparison with the structural dimensions (diameter of droplets and spacing of droplets) for the emulsion. Such effects can be used to probe the diffusive boundary and reveal the microstructure of the emulsion. When motion of a molecule is confined within a restricted spherical geometry such as an emulsion droplet, the limiting mean square displacement in one dimension is correlated with the size of the sphere via Eq. 3.15 [57].

$$
\left\langle Z^{2}\right\rangle=\frac{2}{5} r^{2}
$$

where $r$ is the radius of the sphere. 
When restricted diffusion occurs, the diffusion coefficient depends on the observation time, $\Delta$, and one then measures an apparent diffusion coefficient given by

$$
D_{a p p}=\frac{\left\langle Z^{2}\right\rangle}{2 \Delta}
$$

Further, the restricted diffusion of molecules within a droplet is superimposed on the unrestricted movement of the droplet itself. Because of the widely differing diffusion rates of molecules and droplets, these two diffusions may occur over very different length scales and therefore correlate to different time scales in the NMR experiment. Finally, the interactions between droplets may significantly affect the diffusion coefficient values measured by the spectrometer. Thus, PFGNMR may provide information regarding the solution dynamics.

For the investigation of oil/Triton X-100/water emulsions where the oil is toluene or $p$-xylene, the pulsed gradient stimulated echo technique is preferred due to the short value of the spin-spin relaxation constant $T_{2}$ of our model emulsions ( 200 ms for both toluene and p-xylene emulsions with $50 \mathrm{wt} \%$ oil) compared to the spin-lattice relaxation constant $T_{1}(\sim 3.5 \mathrm{~s}$ for both toluene and $p$-xylene emulsions with 50 wt $\%$ oil). The $T_{1}$ and $T_{2}$ relaxation constants were measured using an inversion recovery sequence [58] and a Carr-Purcell-Meiboom-Gill (CPMG) sequence [59], respectively. The inversion recovery experiment consists of a $180^{\circ}$ pulse followed by a $90^{\circ}$ pulse after a variable time $\tau$. As $\tau$ gets longer, longitudinal relaxation occurs and the net magnetization detected along the horizontal axis decreases, passes through zero after a time corresponding to half of $T_{1}$ and increases again. Additionally, the CPMG pulse sequence comprises a $90^{\circ}$ pulse followed by a series of $180^{\circ}$ pulses. The time interval between two consecutive $180^{\circ}$ pulses is $2 \tau$ which equals twice the time between the $90^{\circ}$ pulse and the first $180^{\circ}$ pulse. Echoes are observed midway between the $180^{\circ}$ pulses. The amplitude of successive echoes decays exponentially with a time constant $T_{2}$. For the study of oil/Triton X-100/water emulsions where the oil is toluene or $p$ xylene, all PFG-NMR experiments were carried out using a Bruker Avance-460 NMR system fitted with a diffusion probe. Calibration was performed using 
Milli-Q water with a measured diffusion coefficient of $2.3 \times 10^{-9} \mathrm{~m}^{2} \mathrm{~s}^{-1}$ at $298 \mathrm{~K}$ [60]. Emulsion samples were placed in $3 \mathrm{~mm}$ tubes to limit the effects of radiation damping, i.e. the broadening of peaks due to strong interactions between the sample magnetization and the induced current in the coil. Emulsions were inserted into the spectrometer about 25 min after emulsification. Only a very small sample volume is necessary $(\sim 0.1 \mathrm{~mL})$ and the sample height in the NMR tube must be adjusted so that the coils are sensitive to the entire sample volume. This is very important when creaming occurs in the sample. The available range of observation time, $\Delta$, is $6.3 \mathrm{~ms}$ to $3 \mathrm{~s}$. The gradient amplitude is typically varied between 1 and $6.5 \mathrm{~T} \mathrm{~m}^{-1}$ and the gradient duration between 2.1 and $4.1 \mathrm{~ms}$. The range of diffusion coefficients able to be probed spans from $\sim 1.0 \times 10^{-14} \mathrm{~m}^{2} \mathrm{~s}^{-1}$ up to $\sim 2.5 \times 10^{-9} \mathrm{~m}^{2} \mathrm{~s}^{-1}$.

A plot of the logarithm of the echo attenuation signal as a function of the product $\gamma^{2} g^{2} \delta^{2}\left(\Delta-\frac{\delta}{3}\right)$ (termed the b value) for pure MilliQ water is illustrated in Figure 3.14. The plot shows a single exponential decay with a slope equal to $-D_{\text {water }}$ as expected from Eq. 3.13. The self diffusion coefficient for pure water is measured to be, $D_{\text {water }}=(2.2 \pm 0.1) \times 10^{-9} \mathrm{~m}^{2} \mathrm{~s}^{-1}$ (this compares well with what measured by Mills, $\left.D_{\text {water }}=2.3 \times 10^{-9} \mathrm{~m}^{2} \mathrm{~s}^{-1}[60]\right)$.

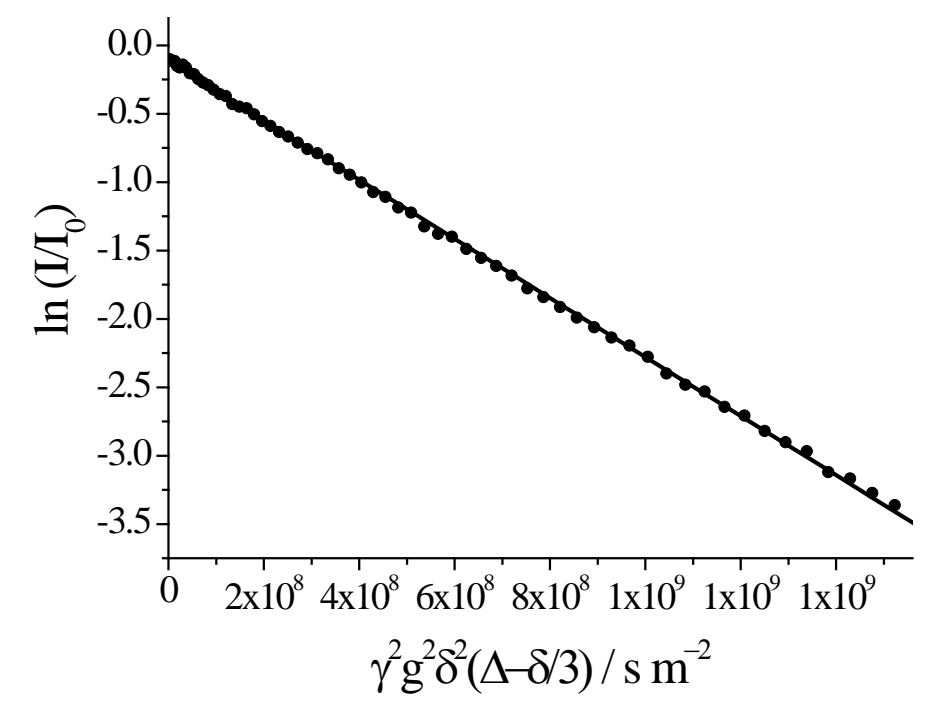

Figure 3.14. Echo attenuation for pure MilliQ water, for an observation time, $\Delta$, of $20 \mathrm{ms,} T$ $=298 \mathrm{~K}$. 
In emulsion samples, molecules in both the continuous phase and inside the droplets as well as droplets of various sizes contribute to the overall echo signal attenuation. Each motion is characterized by its own diffusion coefficient (see Eq. 3.12). As such the echo attenuation follows a multiexponential decay as a function of the $b$ term (Figure 3.15).

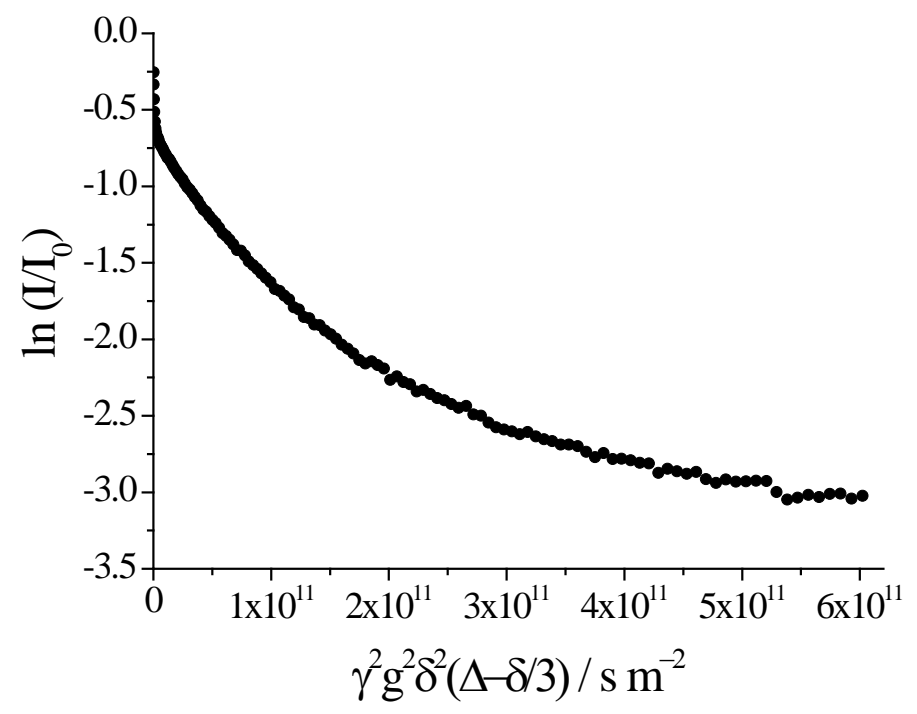

Figure 3.15. Echo attenuation of the oil signal in a toluene/Triton $X-100 /$ water emulsion prepared with $40 \mathrm{wt} \%$ toluene and $8 \mathrm{wt} \%$ Triton $\mathrm{X}-100, \Delta=40 \mathrm{~ms}$.

The distribution of diffusion coefficients (Figure 3.16) of the emulsion sample is extracted from the multiexponential decay data using a one-dimensional inverse Laplace transform, according to an algorithm first used by Provencher [61], using software developed by Callaghan et al. for a 2D inverse Laplace transform [62]. A nonnegative least-squares fit weighted by an additional regularisation function is applied to the exponentially decaying signal. The best fit of the experimental raw data results from the minimisation of $\chi^{2}$, defined as the sum of the squared residuals (least squares method), in addition to a regularisation function which controls the sharpness of the diffusion spectrum via a smoothing parameter $\alpha$. The minimisation of the sharpness of the diffusion spectrum through the appropriate choice of the smoothing parameter $\alpha$ eliminates spurious peaks and provides a better fitting of the multiexponential data. As a consequence this algorithm never provides a sum of well-resolved delta functions. 


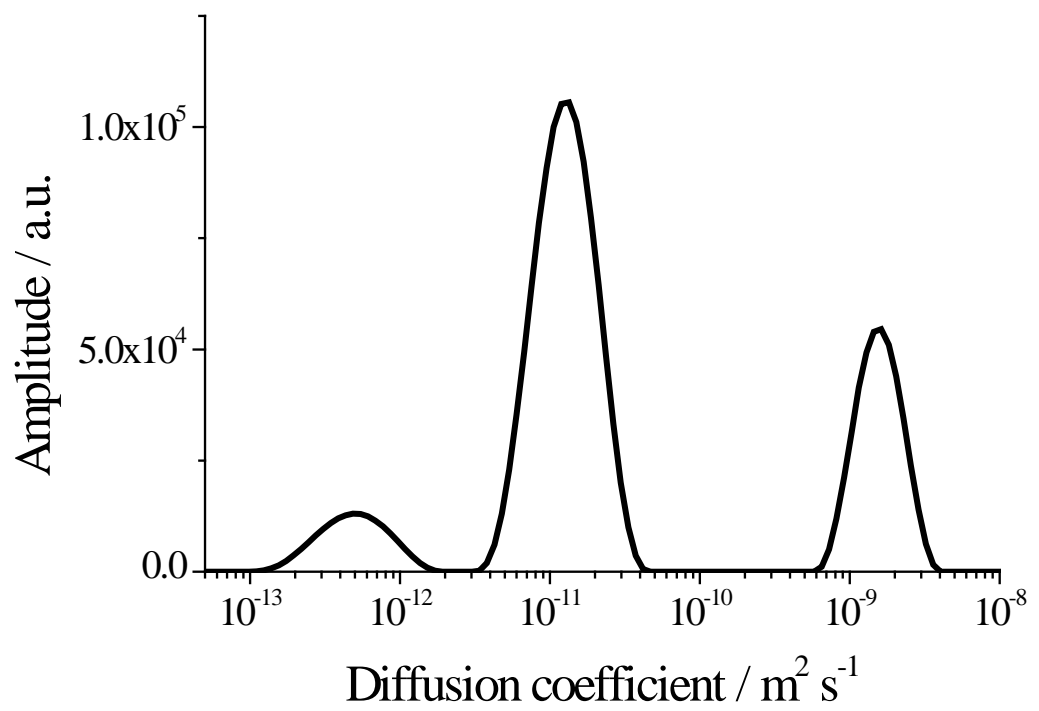

Figure 3.16. Diffusion spectrum of the oil signal in a toluene/Triton $X-100 /$ water emulsion prepared with $40 \mathrm{wt} \%$ toluene and $8 \mathrm{wt} \%$ Triton $X-100, \Delta=40 \mathrm{~ms}$.

Considering the uncertainties associated with the diffusion coefficients given by the inverse Laplace transform, these are calculated from reproducibility. For each observation time, the standard deviation of a given diffusion coefficient was calculated based on repeated experiments. The average value was considered as the best estimate with an uncertainty equal to the standard error or standard deviation of the mean, corresponding to a $68 \%$ confidence interval.

\subsubsection{Laser Scanning Confocal Microscopy}

\subsubsection{Introduction}

The principle of confocal imaging was patented in 1957 by Marvin Minsky [63]. It consists in creating sharp images of thick samples that would appear blurry when viewed with conventional wide-field microscopes [64] as illustrated in Figure 3.17. 

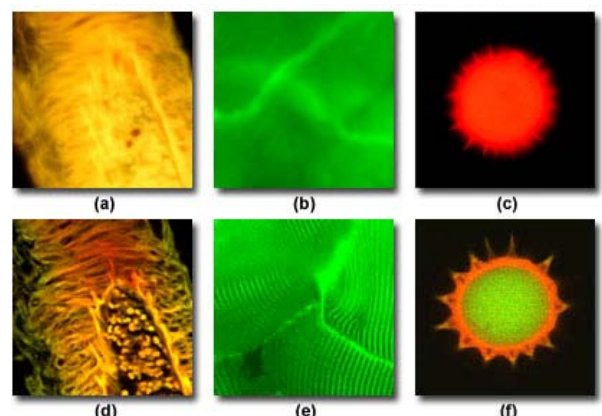

Figure 3.17. Comparison of wide field (top pictures) and confocal (bottom pictures) imaging, (a) and (d) human medulla, (b) and (e) rabbit muscles fibres, (c) and (f) sunflower pollen grain taken from reference [65].

Confocal microscopy also allows a three-dimensional reconstruction of a volume of the sample by assembling a series of thin optical cross-sections taken along the vertical axis; this method is widely used in biological applications [66-68].

For the study of emulsions, it is interesting to monitor droplet interactions [69, 70] and track the droplet dynamics using fluorophores [71, 72] soluble in the dispersed phase and collect optical cross-sections over time at very fast frame rates.

\subsubsection{Experimental equipment}

For the investigation of the dynamics of our emulsions, the confocal system used is an Olympus FV1000 mounted on an IX81 inverted microscope $(\times 100$ objective lens) operating with a multi-line Ar laser (visible light).

The sample is illuminated point-by-point using a pinhole aperture conjugated to the illuminated point in front of the light source and a series of rotating mirrors in order to exclude any scattered light from other points in the sample (Figure 3.18). The intensity of the collected light is weak, therefore an intense light source such as a laser is required. Light is then emitted off the sample via either reflection or fluorescence; the latter emission mode is generally preferred where dye molecules fluorescing at distinct wavelengths are attached to different positions in the sample. Finally, the light that is not from the objective lens's focal plane is rejected using a screen with a pinhole (Figure 3.18) to increase image resolution, and a computer builds an $\sim 1 \mu \mathrm{m}$ thick optical cross-section with a series of 
available frame rates (from $428 \mathrm{~ms}$ to $7 \mathrm{~s}$ for a $256 \times 256$ picture with the Olympus FV1000).

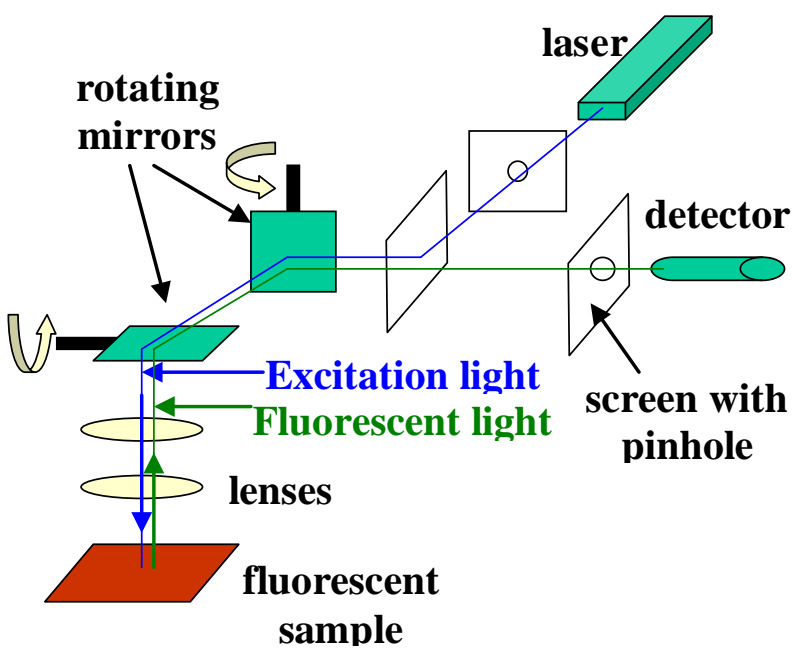

Figure 3.18. Principle of confocal microscopy. Light is emitted by a laser and is scanned across the sample by rotating mirrors. Fluorescent light emitted off the sample that is not from the objective lens's focal plane is rejected by a screen and a pinhole.

Emulsions were prepared with the addition of one of two fluorescent dyes. For red fluorescing dyes: Nile red $\left(\lambda_{\mathrm{ex}}=559 \mathrm{~nm}, \lambda_{\mathrm{em}}=603 \mathrm{~nm}\right)$ and BODIPY 665/676 ((E,E)-3,5-bis-(4-phenyl-1,3-butadienyl)-4,4-difluoro-4-bora-3a,4adiaza-s-indacene) were initially dissolved in the oil at a concentration of $2.7 \times$ $10^{-4}$ wt\% whereas for green fluorophores 4-(4-methoxybenzylamino)-7nitrobenzofurazan $\left(\lambda_{\mathrm{ex}}=473 \mathrm{~nm}, \lambda_{\mathrm{em}}=529 \mathrm{~nm}\right)$ and BODIPY 493/503 (4,4difluoro-1,3,5,7,8-pentamethyl-4-bora- 3a,4a-diaza-s-indacene) were used. The purity of all dyes was $>98 \%$. Nile red and 4-(4-methoxybenzylamino)-7nitrobenzofurazan were purchased from Sigma-Aldrich, the BODIPY dyes from Invitrogen. Samples were sandwiched between a microscope slide and a glass cover slip. The edges of the cover slip were sealed to prevent evaporation due to the high vapour pressure of the aromatic oils used. 


\subsubsection{Droplet dynamics}

The fast dynamics of the oil droplets in our samples could be tracked by imaging two separate emulsions stained with a red fluorescing (Figure 3.19a) and a green fluorescing dye (Figure 3.19b), respectively. A third sample is obtained by simply mixing the previous two samples (Figure 3.19c). In the third sample, all oil droplets emit only yellow fluorescence with no phase separation and no change in the droplet size distribution revealing exchange of the oil molecules on a time scale faster than the experimental set up.

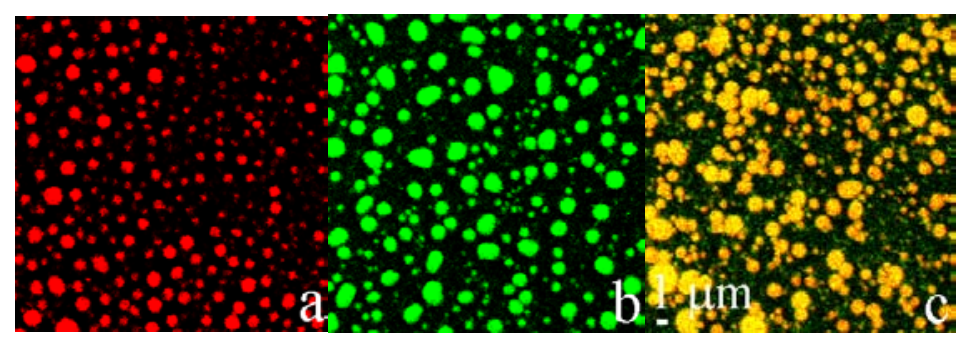

Figure 3.19. 2D confocal snapshots $256 \times 256(\times 100$ objective) of water/Triton $X-100 /$ toluene emulsions prepared with $15 \mathrm{wt} \%$ toluene and 3 wt\% Triton X-100. (a) Emulsion labelled with a red fluorophore. (b) Emulsion labelled with a green fluorophore. (c) Mixture of both emulsions (a) and (b).

\subsubsection{Cryo Scanning Electron Microscopy}

\subsubsection{Introduction}

Cryo scanning electron microscopy (cryo-SEM) allows the imaging of a sample surface by scanning it with a high-energy beam of electrons, which interacts with the atoms that make up the sample producing signals that contain information about the sample's surface topography, composition and other properties. A cryo scanning electron microscope consists of a standard SE microscope with a cryo attachment allowing imaging of liquid samples such as emulsions or biological samples that would otherwise vaporize in the vacuum of the microscope chamber [73]. 


\subsubsection{Experimental equipment}

For the study of emulsions, a Jeol JSM_6500F fitted with a Gatan Alto 2500 cryo attachment (Figure 34) was used. The sample is mounted on the sample holder and plunged into "slushy" nitrogen, i.e. a mixture of solid and liquid nitrogen at a constant temperature of $-210^{\circ} \mathrm{C}$. The mixture of solid and liquid nitrogen has a greater refrigerant capacity than straight liquid nitrogen due to the absence of the Leidenfrost effect, i.e. any liquid boils immediately on contact with an object significantly above its boiling point, enveloping the object in insulating gas. Freezing is generally achieved within $1 \mathrm{~s}$. The sample holder is then withdrawn, under vacuum, into a transfer device for transfer to the cryo-preparation chamber. After transfer to the (separately pumped) cryo-preparation chamber the sample is maintained at a low temperature of $-130^{\circ} \mathrm{C}$ and low contamination conditions. The sample is fractured using a cold knife to expose the internal structure. Finally a thin $(\sim 10 \mathrm{~nm})$ conductive coating of carbon is applied to make the sample conductive and allow high resolution imaging. Transfer to the microscope chamber is via an interlocked airlock and onto a cold stage module fitted to the microscope stage maintained at a temperature of $-130^{\circ} \mathrm{C}$ under high vacuum conditions (typically $\sim \mathrm{p}=9 \times 10^{-5} \mathrm{~Pa}$ ). An electron beam is emitted from a heated tungsten filament (electron gun) and is accelerated by an anode acquiring an energy up to $40 \mathrm{keV}$ (Figure 3.21). The electron beam is focused by a series of condenser lenses under vacuum conditions and is scanned horizontally across the sample by scanning coils. The electron beam interacts with the electron cloud of the sample atoms and scattering takes place. The scattered electrons are detected to form an image. The SEM resolution is between 1 and $5 \mathrm{~nm}$ depending on the type of sample. A wide range of magnification up to $\times 500,000$ is available. 


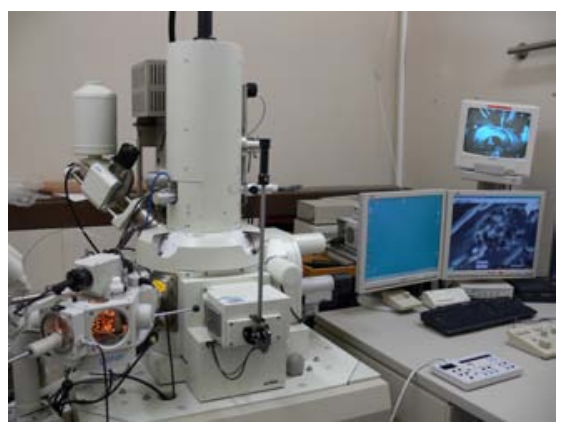

Figure 3.20. Cryo scanning electron microscope from Jeol Gatan.

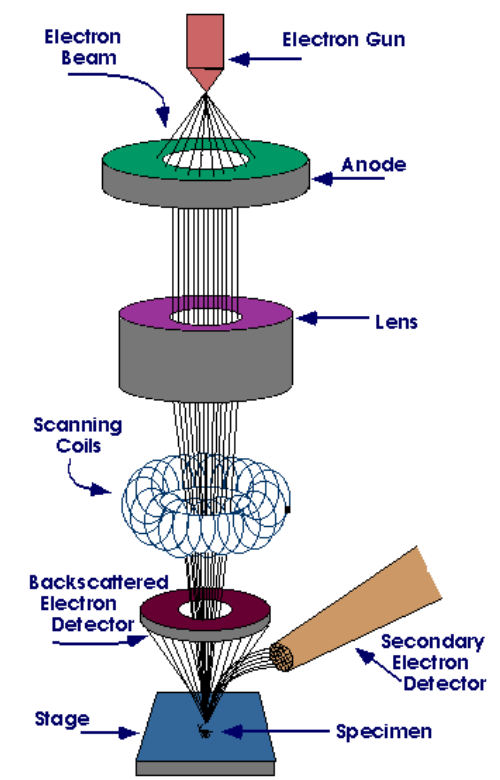

Figure 3.21. Principle of SEM. An electron beam, generated by an electron gun, is accelerated by an anode, focused by a series of lenses and scanned across the sample using scanning coils. The electron beam is scattered by the sample atoms and detected in backscattered or secondary electron mode.

\subsubsection{Image analysis}

Three kinds of detection are available in SEM including secondary electrons, backscattered electrons and X-rays [74] corresponding to three different interactions between the electron beam and the atoms (Figure 3.22).

The first interaction generates secondary electrons which are outer-shell electrons ejected from the sample as a result of inelastic scattering. This scattering occurs within a very small depth, typically between 1 and $2 \mathrm{~nm}$ reflecting the topography (surface structure) of the sample. The second interaction is the elastic scattering of the incident electrons by the atomic nuclei through more than $90^{\circ}$. This scattering 
takes place deeper within the sample between 10 and $100 \mathrm{~nm}$ and is proportional to the square of the atomic number. The information obtained from this interaction is both an atomic number and orientation contrast. The third interaction is the inelastic scattering of the incoming electrons by inner-shell electrons generating characteristic X-ray photons by relaxation. The chemical composition of the sample can be obtained by energy-dispersive spectroscopy (EDS) or wavelength-dispersive spectroscopy (WDS).

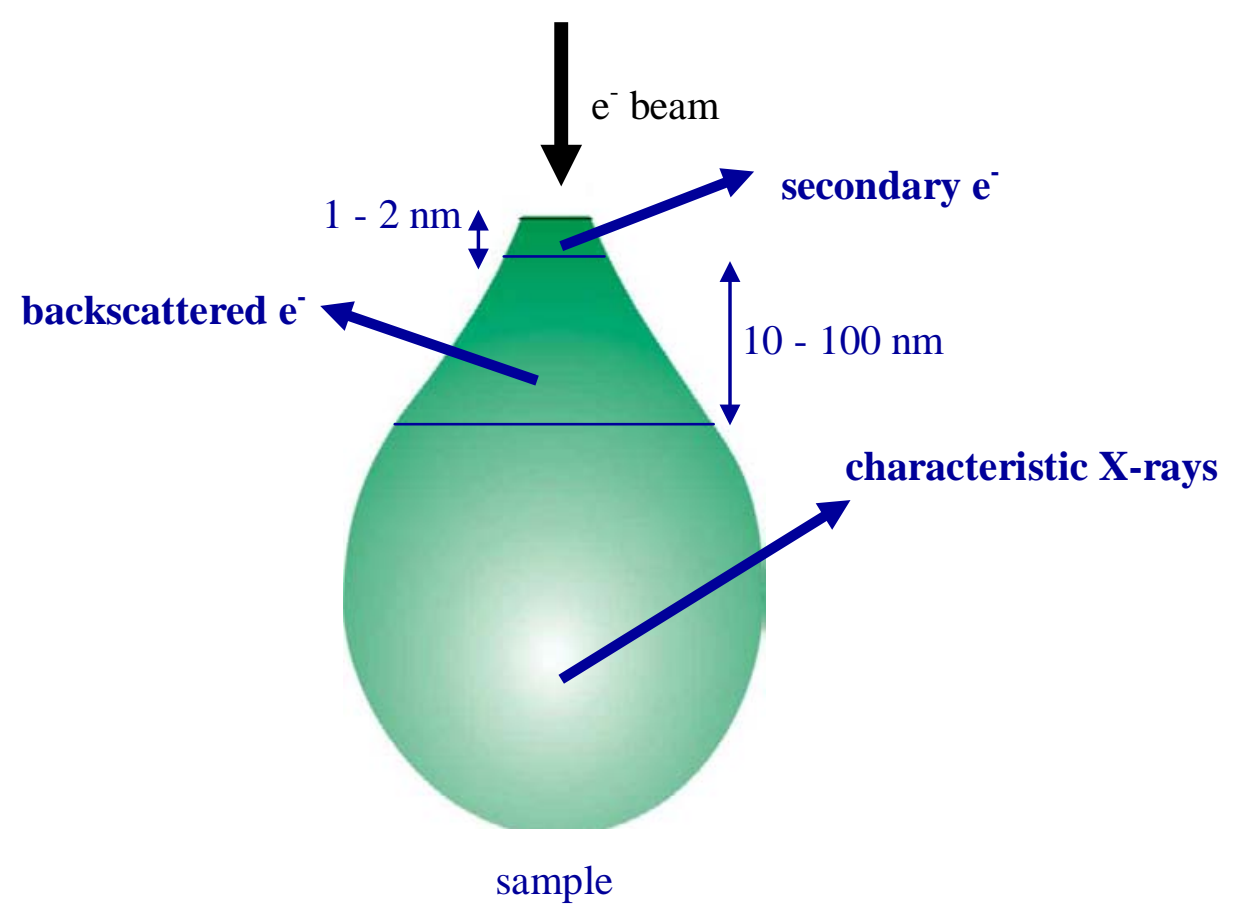

Figure 3.22. Volume of interaction between the electron beam and the sample. Secondary electrons are generated by scattering of the outer-shell electrons. Backscattered electrons are directly scattered by the atomic nuclei whereas characteristic $X$-rays result from the scattering of the incoming electrons by inner-shell electrons upon relaxation.

Although giving valuable information, the latter two modes of detection, i.e. backscattered electrons and X-rays cannot be used for the study of our emulsions since the atomic number of the constituents are too low. 


\subsubsection{Rate of macroscopic phase separation}

\subsubsection{Introduction}

Macroscopic phase separation experiments consist of observing the destabilization of an emulsion over time and assessing how fast the aqueous continuous phase is expelled from the emulsion by measuring the volume of the expelled phase relative to the total sample volume. In this investigation, the rates of macroscopic phase separation are evaluated by placing samples in $3 \mathrm{~mm}$ diameter NMR tubes and measuring over time the height of expelled aqueous phase relative to the total height of the sample (Figure 3.23).

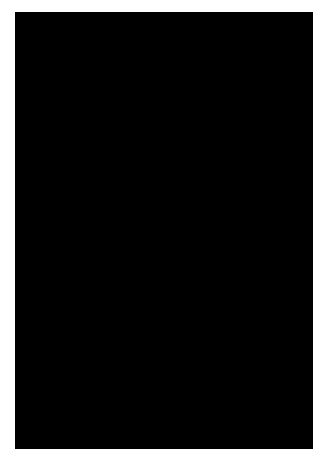

Figure 3.23. Evaluation of the rate of macroscopic phase separation of a toluene/Triton X100/water emulsion, here $15 \mathrm{wt} \%$ oil.

\subsubsection{Data analysis}

In order to evaluate the relative stability of emulsions as a function of emulsion characteristics such as oil concentration, nature of the oil, age, energy input etc, a plot of the percentage of expelled phase as a function of time is drawn as illustrated in Figure 3.24. Comparing plots enables the assessment of how a particular factor affects emulsion destabilisation. 


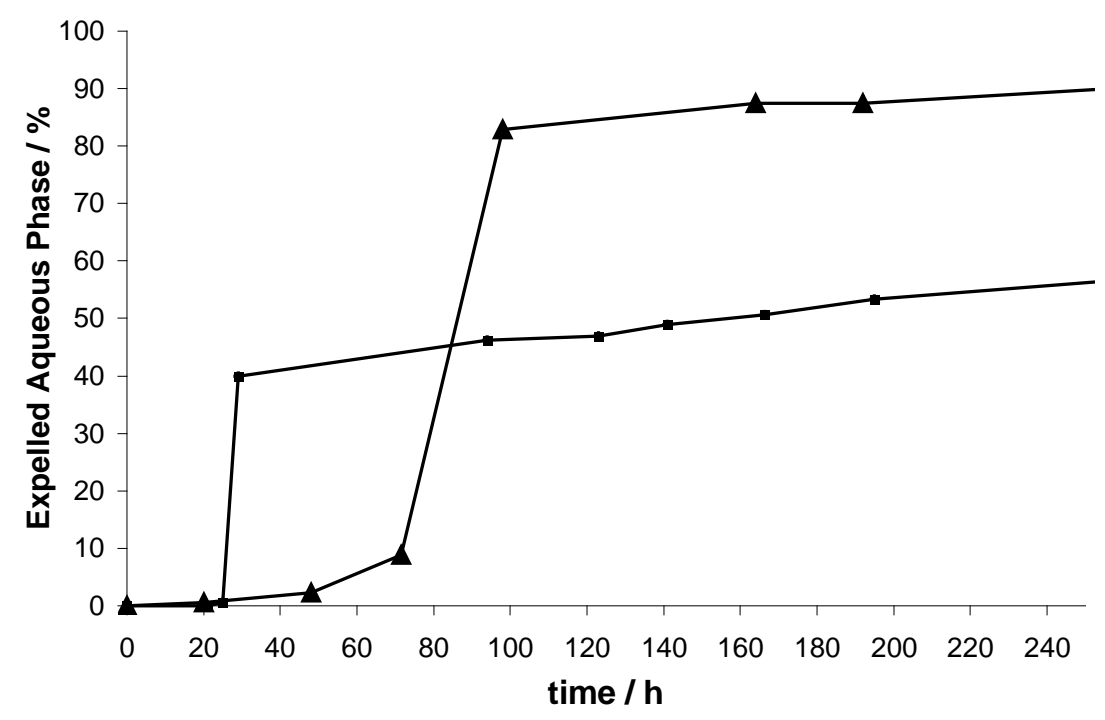

Figure 3.24. Rate of macroscopic phase separation for a toluene/Triton X-100/water emulsion prepared with 15 wt\% (triangles) and 35 wt\% toluene (squares).

\subsubsection{Surface charge}

\subsubsection{Introduction}

Most liquids contain ions and when a charged particle such as a colloidal particle is suspended in a liquid, counter ions are attracted to the surface of the suspended particle. An electrical double layer is formed around each particle [3] (Figure 2.7). The electrical double layer consists of an inner region, called the Stern layer, where the ions are strongly bound and an outer region, called the diffuse layer where they are loosely attached. Within the diffuse layer there is a notional boundary called the surface of hydrodynamic shear or slipping plane inside which the ions and particles form a stable entity. When a particle moves, ions within the slipping plane move with it, but any ions beyond the boundary do not travel with the particle. A potential exists between the particle surface and the double layer, which varies according to the distance from the particle surface, the potential at the slipping plane is called the zeta potential. The zeta potential indicates if colloidal particles are charged or non-charged and the magnitude gives a measure of the stability of the system. A large negative or positive zeta potential results in the repulsion of the particles among themselves and a weak tendency to 
flocculate. Colloidal systems with low zeta potential (typically $\mid \xi_{\text {potential }} \leq 30 \mathrm{mV}$ ) are unstable with respect to electrostatic repulsion [75]. The $\mathrm{pH}$ value is an important factor affecting the zeta potential.

\subsubsection{Principle of zeta potential measurement}

For the determination of the zeta potential of emulsion droplets, a Zetasizer nanoZS from Malvern Instruments was used. In a similar way to the typical dynamic light scattering system described in section 3.4.2, the zeta-potential measurement system comprises six main components. A laser provides a coherent intense light source to illuminate the particles within the sample. The light source is split to provide an incident and reference beam. An attenuator placed in front of the light source adjusts the intensity of the laser. The laser beam passes through the centre of a folded capillary cell and the scattering at an angle of $17^{\circ}$ is detected. When an electric field is applied across a liquid, charged particles suspended in the liquid are attracted towards the electrode of opposite charge (Figure 3.25). Viscous forces on the particles oppose this movement. When equilibrium is reached between these two opposing forces, the particles move with constant velocity.

\section{Electrode}

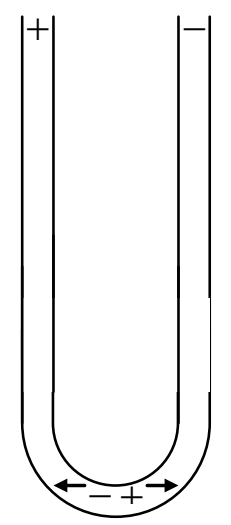

Figure 3.25. Folded capillary cell used in an electrophoresis experiment.

Any particles moving through the measurement volume will cause the intensity of light detected to fluctuate with a frequency proportional to the particle velocity. A digital signal processor is used to extract the characteristic frequencies in the 
scattered light. This information is then passed to a computer, where the Zetasizer Nano software produces a frequency spectrum from which the electrophoretic mobility (velocity of a particle in an electric field) is calculated. Henry's equation relates the electrophoretic mobility to the zeta potential for spherical particles [75](Eq. 3.17).

$$
U_{E}=\frac{2 \varepsilon \xi f\left(K_{a}\right)}{3 \eta}
$$

where $U_{E}$ is the electrophoretic mobility, $\varepsilon$ is the dielectric constant, $\zeta$ is the zeta potential, $\eta$ is the viscosity and $f\left(K_{a}\right)$ is Henry's function. When the measurement is made in aqueous media and moderate electrolyte concentration, $f\left(K_{a}\right)$ equals 1.5 whereas the function equals 1 for non-aqueous media.

Once the electrophoretic mobility of the particles and the applied electrical field are known, the zeta potential can be determined by using the two other known constants of the sample (viscosity and dielectric constant).

\subsection{Emulsion characterisation}

Emulsions are by nature kinetically stable as opposed to thermodynamically stable. As such, emulsion microstructure, stability and dynamics strongly depend on the relative weight composition of the constituents, the method of formulation, the energy input and the temperature. In the study of toluene/Triton X-100/water emulsions, the method of formulation, the energy input and the temperature were kept constant. Oil concentration was varied between 5 and 70 wt\% (a 5:1 oil-tosurfactant weight ratio was maintained) as well as the nature of the oil by using $p$ xylene or octane instead of toluene. Measurement of the zeta potential indicated that the charge at the surface of the toluene droplets was weak. This confirmed that electrostatic repulsive forces could be ignored when considering the stability of these emulsions as expected. Only steric forces and structural forces in addition to van der Waals forces needed be considered. Both SLS and DLS were used to determine the droplet size distribution as a function of oil concentration, the former is appropriate for droplet size in the micrometer range while the latter is more sensitive to droplet size in the nanometer range due to the range of 
scattering angles that can be detected. Moreover, these techniques allow droplet size to be monitored as a function of time in order to determine destabilization mechanisms such as coalescence and Ostwald ripening. Both techniques are easy to use and require no sample preparation. However it is essential to keep in mind that they both require significant dilution which may affect emulsion microstructure.

On the other hand, confocal microscopy was appropriate to probe droplet interactions via the fluorescence of two distinct hydrophobic dyes solubilised within oil droplets. Then, the droplet size distribution acquired via confocal images could be compared with that measured by SLS.

Moreover, PFG-NMR is a unique tool to gain information regarding emulsion microstructure and oil dynamics over very fast time scales (from $\sim 6 \mathrm{~ms}$ up to $3 \mathrm{~s}$ ) as a function of both oil concentration and emulsion age. The diffusion coefficients extracted from the echo attenuation as a function of time (up to $3 \mathrm{~s}$ ) are associated with different motional rates of the oil phase and correlate to specific oil environments. Droplet interactions were probed and the time constant of the oil dynamics quantitatively determined. In addition to probing very fast dynamics of emulsion systems, PFG-NMR presents many advantages. It is a noninvasive technique ensuring that no external perturbation is applied to the sample which is of significant importance when investigating kinetically stabilized systems. Moreover the experimental procedure is fully automatic, i.e. once a sample is inserted into the NMR spectrometer, a pulse sequence can be repeated automatically for several days without further sample manipulation.

Additionally, cryo-SEM was used to image the internal microstructure of emulsion samples at very high magnification and resolution. This gave us insight about the interfacial domain microstructure and the droplet interactions.

Emulsion stability is an essential property of kinetically stabilized systems. Consequently, measurement of the rate of macroscopic phase separation as a function of oil concentration is a straight forward way to assess emulsion stability over time and identify possible concentration domains which detabilize via different mechanisms. 


\section{Chapter 4 Oil exchange in toluene/Triton $\mathrm{X}-100 /$ water emulsions}

\subsection{Introduction}

Emulsions are highly dynamic systems due to the occurrence of Brownian motion and destabilisation processes such as coalescence and Ostwald ripening. In O/W emulsions, the diffusion of oil droplets and that of oil molecules inside droplets are driven by thermal energy. Many industrial applications of $\mathrm{O} / \mathrm{W}$ emulsions include the transport of water-insoluble substances such as colorants, preservatives, drugs, etc. As such, the investigation of emulsions dynamics, e.g. exchange between oil droplets, is of particular interest to determine the distribution of hydrophobic materials within the dispersed phase. Moreover, oil exchange may affect the bulk properties of emulsions such as their rheological behaviour and stability.

Upon Ostwald ripening oil can be transferred from one droplet to another through the continuous phase or aided by surfactant micelles. Oil can also be exchanged between droplets upon coalescence, i.e. the fusion of the interfacial membrane of two or more droplets, or via direct contact of oil domains upon collision.

In this study, evidence for oil exchange between droplets with no simultaneous droplet growth, occurring in oil/Triton X-100/water emulsions where the oil is one of toluene, $p$-xylene or octane, is reported. Exchange takes place either via molecular permeation upon droplet collision or reversible coalescence of the droplets.

In this chapter, the focus was made on the investigation of toluene/Triton X100/water emulsions; the investigation of $p$-xylene and octane emulsions will be presented in the last chapter of the thesis. After describing the toluene emulsion microstructure upon varying oil content, the experimental data referring to oil exchange using laser scanning confocal microscopy, cryo-SEM and PFG-NMR will be described. 


\subsection{Droplet size distribution}

The droplet size distributions of the toluene emulsions upon varying oil content between 5 and $70 \mathrm{wt} \%$ were determined by SLS (Figure 4.1) using a Mastersizer 2000 from Malvern Instruments. The refractive index and absorption coefficient of the dispersed phase were taken as 1.390 and 0.001 , respectively. The dispersant viscosity ( $0.8872 \mathrm{cP}$ for water at $298 \mathrm{~K})$ was taken as the sample viscosity. The droplet size distribution was calculated with the inherent Mastersizer software using Mie theory (section 3.4.1) and assuming the sample is comprised of polydisperse particles with irregular shape.

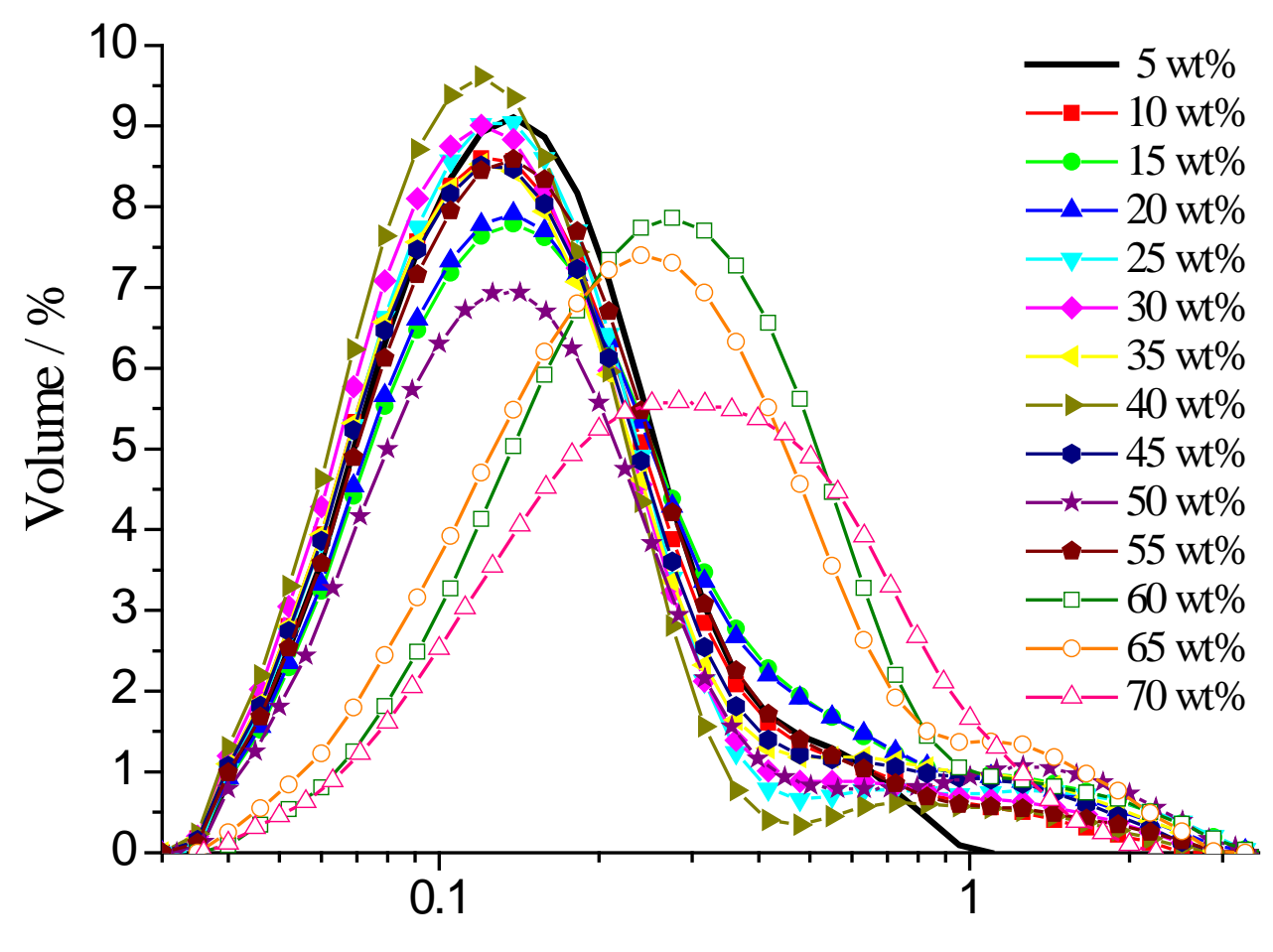

Droplet diameter / $\mu \mathrm{m}$

Figure 4.1. Droplet size distributions of emulsions between 5 and $70 \mathrm{wt} \%$ toluene generated by SLS.

The distributions all exhibit a lognormal shape, i.e. the logarithm of the droplet diameters is normally distributed. The smallest droplet diameters are measured at $\sim 35 \mathrm{~nm}$ and the largest at $\sim 3 \mu \mathrm{m}$ for all toluene concentrations except for the lowest concentration of $5 \mathrm{wt} \%$ oil where the maximum droplet size is measured at $1 \mu \mathrm{m}$. Between 5 and $55 \mathrm{wt} \%$ oil, no dependence of the droplet size on oil concentration is observed; the initial mean droplet diameter is $0.23 \pm 0.10 \mu \mathrm{m}$ and 
the contribution of the smallest droplets of the system dominates with the maximum of the size distribution at $0.15 \pm 0.10 \mu \mathrm{m}$. The largest droplets between $\sim 0.4$ and $3 \mu \mathrm{m}$ represent only a small portion of the sample as illustrated by the number-based droplet size distribution generated by the DLS software (Figure $4.2)$.

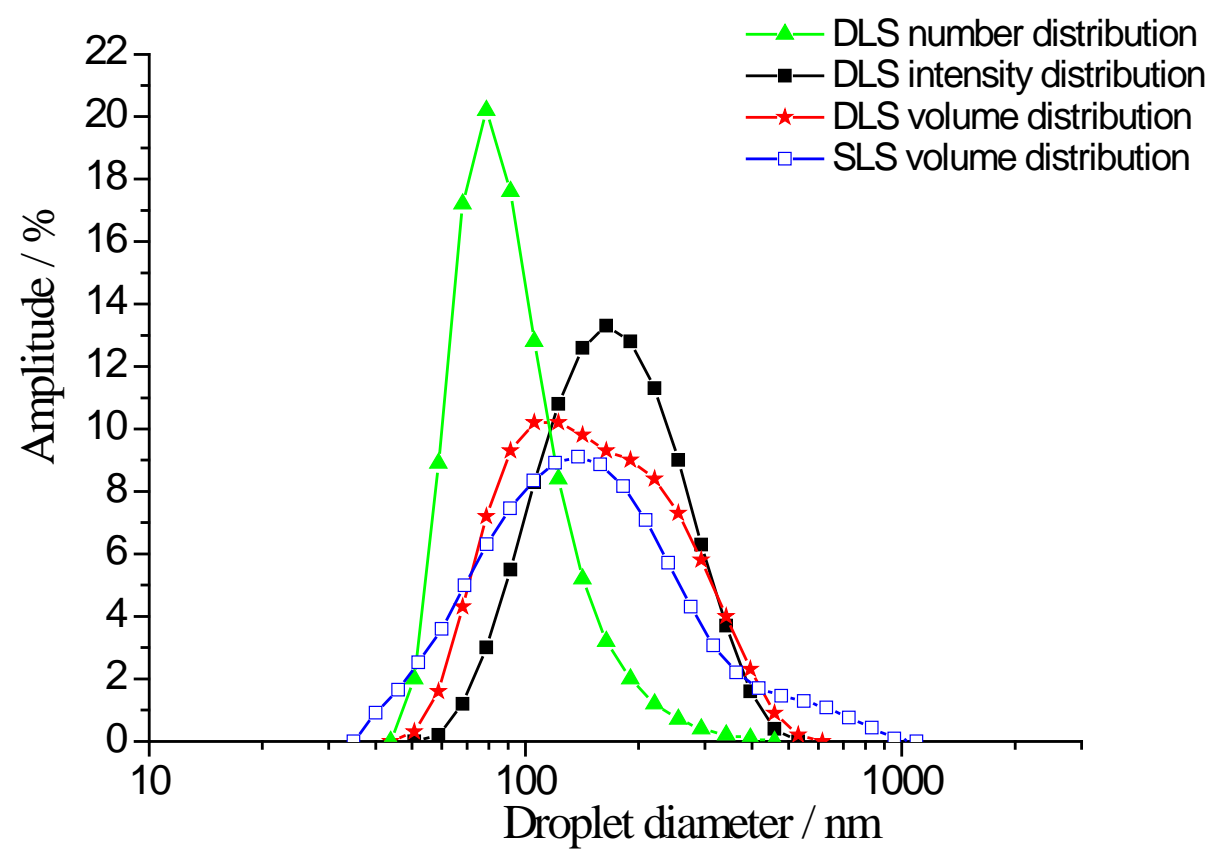

Figure 4.2. Number, intensity and volume droplet size distributions measured by DLS and volume droplet size distribution measured by SLS for a fresh emulsion prepared with $5 \mathrm{wt} \%$ toluene.

The raw data measured by both SLS and DLS are intensity distributions which can be converted to volume distributions using Mie theory or number distributions. Converting the raw intensity distribution of a $5 \mathrm{wt} \%$ toluene sample to a volume distribution using the DLS software results in an apparent decrease in the amplitude of the distribution representing the largest droplets coupled with an apparent increase in the amplitude for the smallest droplets with no change in the range of droplet sizes (Figure 4.2). This is due to the signal amplitude in an intensity distribution being proportional to the sixth power of the particle diameter (Rayleigh's approximation) whereas the signal amplitude in a volume distribution is proportional to the third power of the particle diameter. The volume size distribution of a $5 \mathrm{wt} \%$ toluene sample measured by DLS corresponds well to the volume size distribution measured by SLS although the 
former is slightly narrower. Using DLS, the size range spans from $\sim 40 \mathrm{~nm}$ to $\sim 0.6$ $\mu \mathrm{m}$ with a maximum at $0.17 \pm 0.05 \mu \mathrm{m}$ (Figure 4.2) whereas using SLS, the range is slightly broader spanning from $\sim 35 \mathrm{~nm}$ to $\sim 1 \mu \mathrm{m}$ but the maximum is similar (0.15 $\pm 0.10 \mu \mathrm{m}$, Figure 4.1). Upon comparing the volume and number size distributions of a $5 \mathrm{wt} \%$ toluene sample using DLS, it appears that the proportion of droplets larger than $0.2 \mu \mathrm{m}$ are overestimated in the volume distribution. The mean droplet diameter shifts from $0.17 \pm 0.05 \mu \mathrm{m}$ (volume distribution) to $0.08 \pm$ $0.05 \mu \mathrm{m}$ (number distribution).

For emulsion samples between 60 and $70 \mathrm{wt} \%$ oil, the mean droplet diameter measured by SLS increases from 0.23 to $0.37 \pm 0.10 \mu \mathrm{m}$ and the maximum of the distribution shifts from 0.15 to $0.30 \pm 0.10 \mu \mathrm{m}$ (Figure 4.1). The presence of a closed-cell biliquid foam in addition to the dispersed oil droplet phase may partly explain the observed increased average droplet size. Indeed, a closed-cell foam has already been stabilised at low energy input (vortex mixer) in toluene/Triton $\mathrm{X}-100 /$ water emulsions for oil concentrations larger than $65 \mathrm{wt} \%$ [40]. This structure, more commonly associated with gas/liquid dispersions, shows faceting of large oil domains of average diameter of $\sim 4 \mu \mathrm{m}$ and Plateau borders (Figure 4.3).

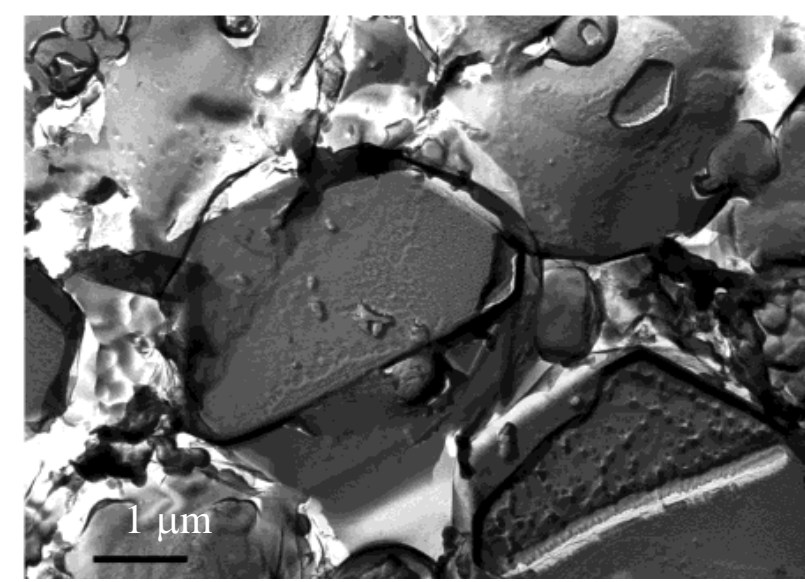

Figure 4.3. TEM micrograph of a closed-cell foam coexisting with a dispersed droplet $\mathrm{O} / \mathrm{W}$ emulsion with $65 \mathrm{wt} \%$ toluene taken from reference [40].

Additionally, for toluene concentrations between 5 and $60 \mathrm{wt} \%$, the presence of oil swollen micelles was detected by DLS (section 3.4.2.). Oil swollen micelles are thermodynamically stable droplets formed in the continuous phase of 
emulsions if there is an excess of surfactant molecules once the surface of the droplets is covered, i.e. a kinetically stabilised emulsion is superposed to a thermodynamically stable microemulsion. Oil swollen micelles were probed in the expelled aqueous bottom phase of a separated emulsion sample (Figure 4.4) using a Zetasizer nano-ZS from Malvern Instruments. The refractive index and absorption coefficient of the dispersed phase were taken as 1.390 and 0.001 , respectively. The dispersant viscosity $(0.8872 \mathrm{cP}$ for water at $298 \mathrm{~K})$ was taken as the sample viscosity.

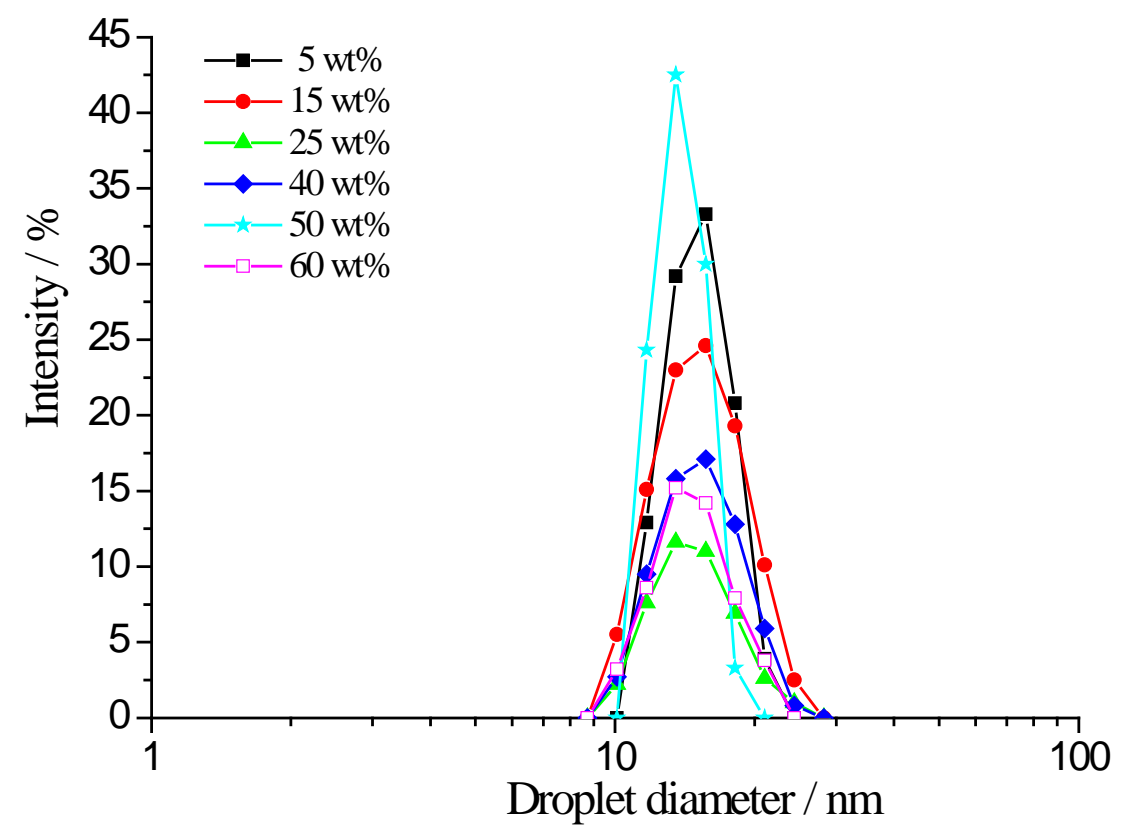

Figure 4.4. Droplet size distributions of the expelled aqueous phase of aged toluene/Triton X100/water emulsions between 5 and 60 wt\% toluene using DLS.

For all concentrations, the distribution shows the presence of oil swollen micelles between $\sim 9$ and $\sim 30 \mathrm{~nm}$. The size distribution has a lognormal shape with a mean diameter at $15 \pm 5 \mathrm{~nm}$. It was not possible to perform similar measurements on highly concentrated emulsions between 65 and $70 \mathrm{wt} \%$ oil due to the small quantity of expressed aqueous phase.

Moreover, the full distribution of oil droplets and oil swollen micelles could be measured simultaneously only for the lowest oil concentration of $5 \mathrm{wt} \%$ after substantial dilution (Figure 4.5). Above this concentration, significant dilution was necessary for the measurement and complete break up of the emulsion sample occurred. 


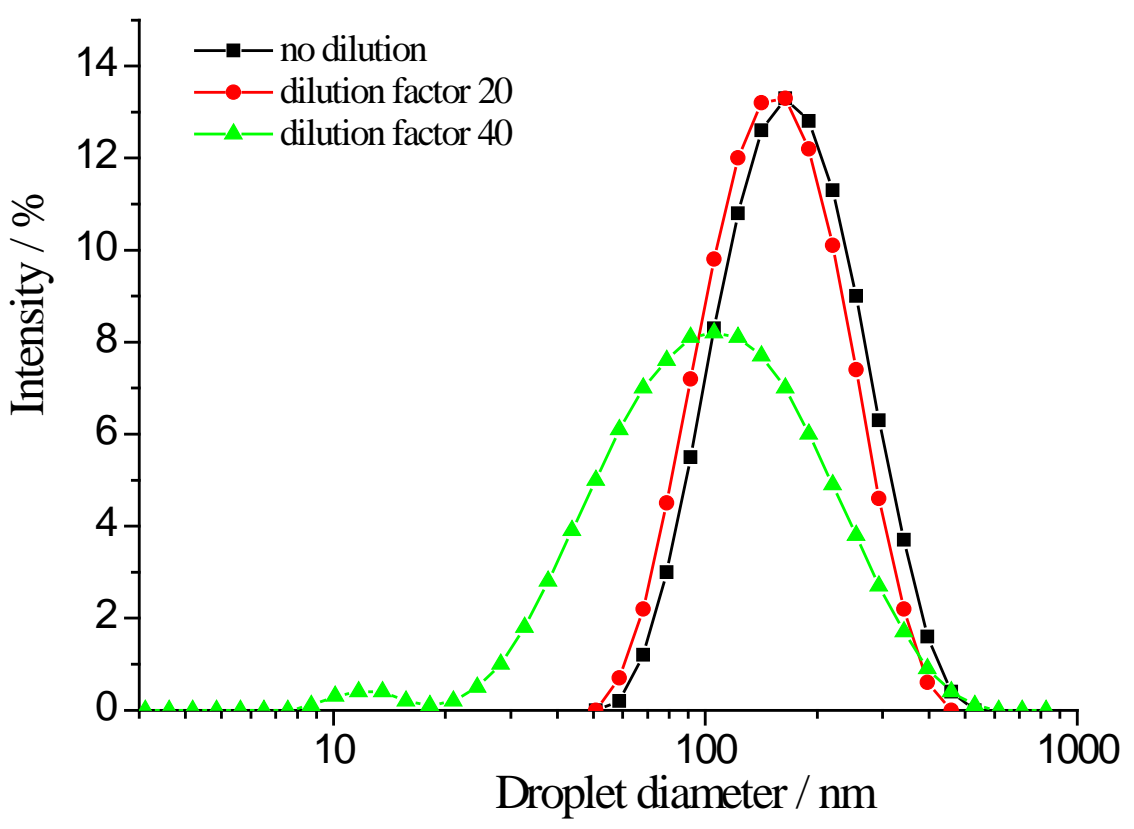

Figure 4.5. Droplet size distributions of diluted fresh toluene/Triton X-100/water emulsions prepared with $5 \mathrm{wt} \%$ toluene using DLS.

Without dilution, there is no sign of the presence of oil swollen micelles in freshly emulsified samples because the signal is swamped by the presence of the larger oil droplets. However, upon dilution above a factor of 40, the size distribution broadens and two distinct peaks are observed. The broad intense peak corresponds to the size of oil droplets between 0.02 and $0.5 \mu \mathrm{m}$ with a maximum at $0.12 \pm 0.05 \mu \mathrm{m}$ while the low magnitude peak confirms the presence of oil swollen micelles between 8 and $20 \mathrm{~nm}$ diameter in the continuous phase of fresh emulsions with a maximum at $13 \pm 5 \mathrm{~nm}$. The presence of oil swollen micelles in fresh emulsions was also detected by diffusion NMR (see section 5.2).

\subsection{Laser scanning confocal microscopy}

Droplet-droplet interactions and the dynamics of oil droplets in oil/Triton X100/water emulsions where the oil is toluene, $p$-xylene or octane were monitored using fluorophores that are soluble in the oil droplets and insoluble in the aqueous continuous phase. Emulsions were prepared with the addition of one of the following fluorescent dyes. Nile red $\left(\lambda_{\mathrm{ex}}=559 \mathrm{~nm}, \lambda_{\mathrm{em}}=603 \mathrm{~nm}\right.$, Figure $\left.4.6 \mathrm{a}\right)$ or BODIPY 665/676 ((E,E)-3,5-bis-(4-phenyl-1,3-butadienyl)-4,4-difluoro-4-bora3a,4a-diaza-s-indacene, Figure 4.6 b) as red fluorescing dyes and 4-(4- 
methoxybenzylamino)-7-nitrobenzofurazan $\left(\lambda_{\mathrm{ex}}=473 \mathrm{~nm}, \lambda_{\mathrm{em}}=529 \mathrm{~nm}\right.$, Figure 4.6 c) or BODIPY 493/503 (4,4-difluoro-1,3,5,7,8-pentamethyl-4-bora-3a,4adiaza-s-indacene, Figure 4.6 d) as green fluorescing dyes.

(a)

(b)

(c)

(d)

Figure 4.6. Chemical formula of Nile red (a), BODIPY 665/676 (b), 4-(4methoxybenzylamino)-7-nitrobenzofurazan (c) and BODIPY 493/503 (d).

The dye solution ( $\mathrm{c}_{\mathrm{dye}}=10^{-4} \mathrm{~g} \mathrm{~mL}^{-1}$ in 1,2-propanediol) was initially added to the oil before emulsification to give a final dye concentration in the oil of $9 \times 10^{-7}$ mol\%. Images were obtained from a 100× objective lens supported by an electronically-adjusted magnification (variation of the scanned area) corresponding to a specific zoom factor. Optical cross-sections $(\sim 1 \mu \mathrm{m}$ thick, $256 \times 256$ pixels) were collected as a function of time at the fastest rate of 428 ms/frame 30 minutes after emulsification.

In this chapter the confocal microscopy data for the toluene/Triton X-100/water system were considered. Figure 4.7 shows a $2 \mathrm{D}$ snapshot for a sample freshly emulsified with 15 wt\% toluene and labelled with Nile red. 


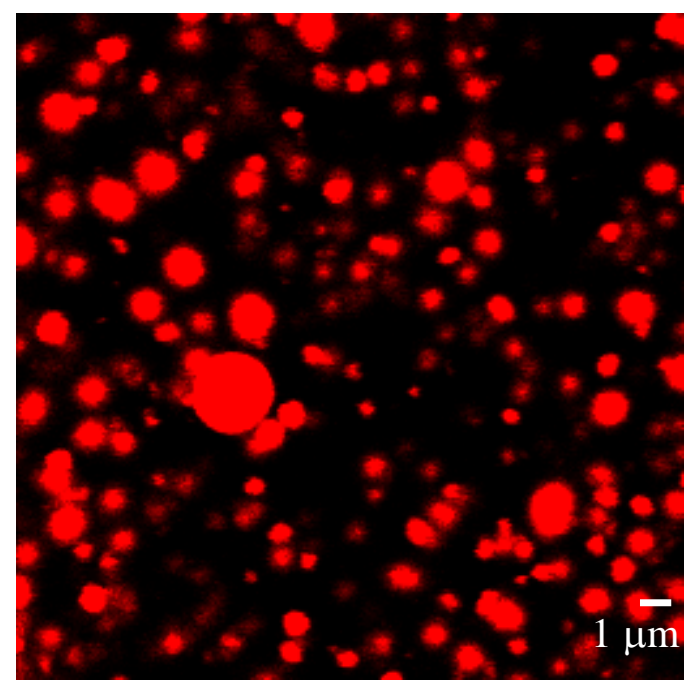

Figure 4.7. 2D confocal microscopy optical cross-section $256 \times 256(\times 100$ objective, zoom factor 4) of a $15 \mathrm{wt} \%$ toluene emulsion sample labelled with Nile red. Snapshot taken 30 minutes after emulsification.

Diameters of most of the droplets are between $200 \mathrm{~nm}$ (resolution of the confocal microscope) and $\sim 1 \mu \mathrm{m}$ although a few $\sim 2 \mu \mathrm{m}$ diameter droplets are apparent. Consequently, the droplet size distribution observed under the microscope for a $15 \mathrm{wt} \%$ toluene emulsion corresponds well with the droplet size distribution measured by SLS with and without dye addition, i.e. droplets are comprised between $\sim 35 \mathrm{~nm}$ and $\sim 3 \mu \mathrm{m}$ including only a small number of droplets larger than $\sim 1 \mu \mathrm{m}$ (Figure 4.8). As such, the presence of dye molecules solubilised in the oil droplets does not affect the droplet size distribution of the toluene emulsions. 


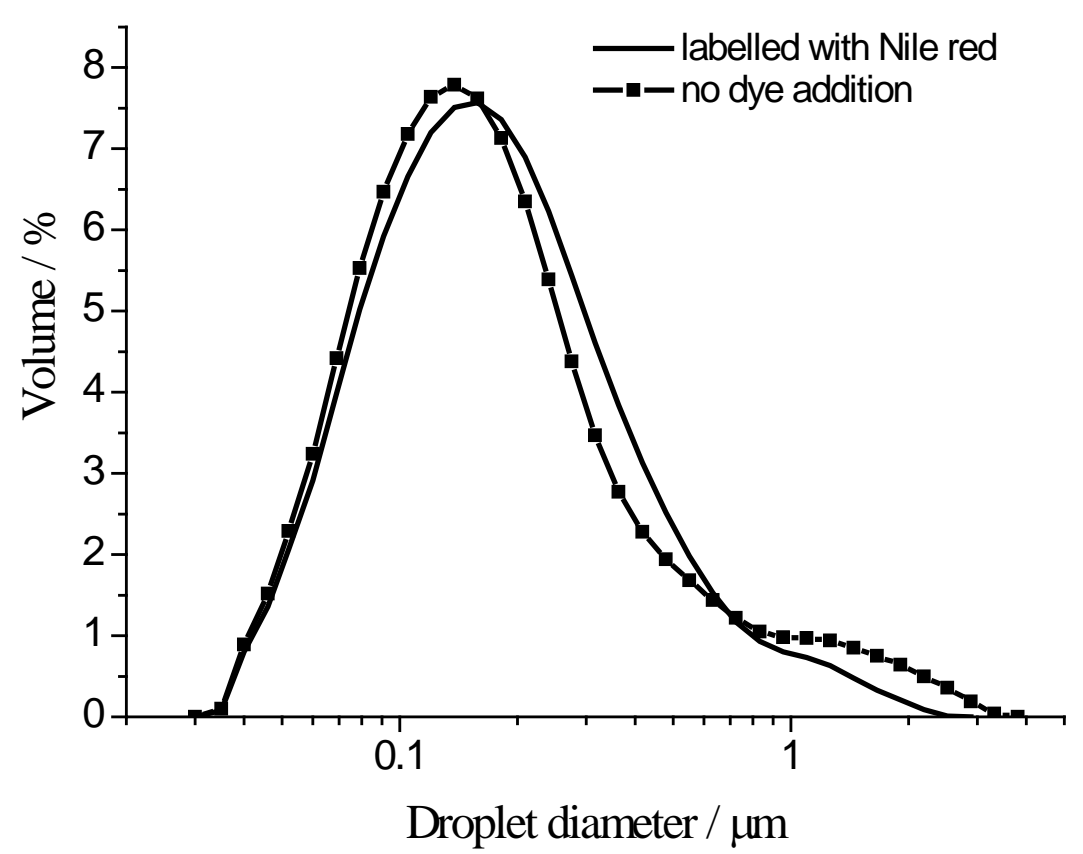

Figure 4.8. Droplet size distribution of a $15 \mathrm{wt} \%$ toluene emulsion sample labelled with Nile red (straight line) and with no dye addition (squares) obtained by SLS.

Additionally, the link in Figure 4.9 provides the optical cross-section collected over time for a freshly emulsified sample prepared with $15 \mathrm{wt} \%$ toluene and labelled with Nile red*.

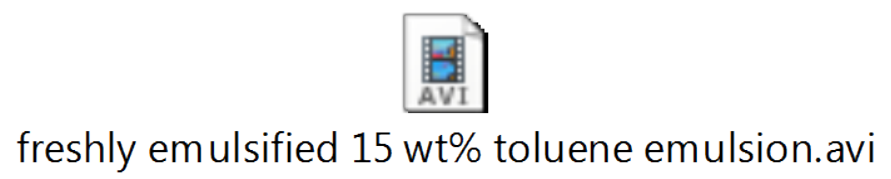

Figure 4.9. Link to optical cross-section collected over time at $428 \mathrm{~ms} / \mathrm{frame}, 256 \times 256$ pixels, zoom factor 4 for a freshly emulsified $15 \mathrm{wt} \%$ toluene emulsion sample labelled with Nile red using confocal microscopy.

From this video, in spite of the poor image resolution, droplets colliding with each other due to Brownian motion were observed. The poor image resolution is caused by the fast frame rate imposed by the dynamics of the emulsion system coupled with significant photobleaching. Photobleaching is the reduction of fluorescence emission intensity as a result of the interaction of the excited dye molecules with oxygen free radicals. It can be reduced by decreasing the scanned area using a smaller zoom factor or by the addition of scavengers that preferentially react with the oxygen free radicals.

\footnotetext{
* Using Windows media player, one may want to change the play speed settings to slow mode under Now playing/Enhancements/Play speed settings to obtain the real time dynamics of the oil droplets.
} 
However, it was impossible to determine whether droplets exchange oil molecules upon direct contact using a single dye. Therefore, two separate emulsions at equal oil concentration, stained with a red fluorescing (Nile red, Figure 4.7) and a green fluorescing dye (4-(4-methoxybenzylamino)-7nitrobenzofurazan, Figure 4.10), respectively, were prepared.

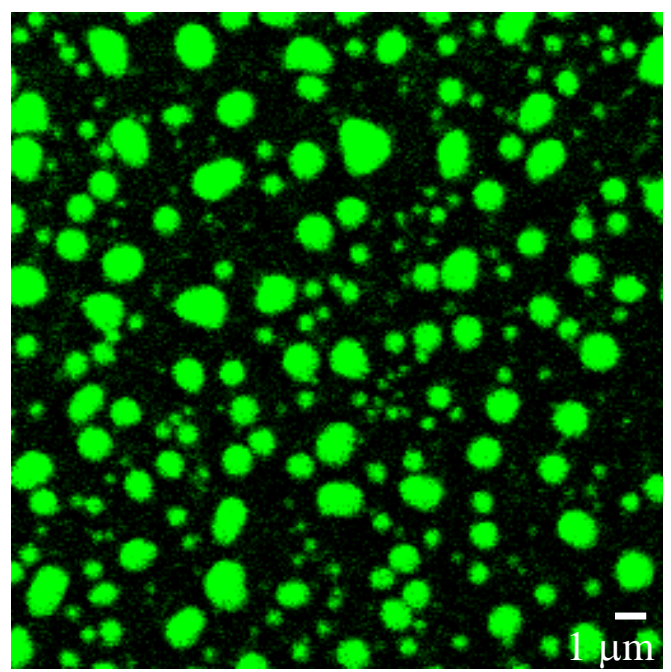

Figure 4.10. 2D confocal microscopy optical cross-section $256 \times 256(\times 100$ objective, zoom factor 4) of a $15 \mathrm{wt} \%$ toluene emulsion sample labelled with 4-(4-methoxybenzylamino)-7nitrobenzofurazan. Snapshot taken 35 minutes after emulsification.

Then, a third sample was prepared by mixing the previous red fluorescing and green fluorescing emulsions. The first emulsion was poured into the second one under gentle hand stirring. The third emulsion was then observed under the confocal microscope within 10 minutes of mixing and 40 minutes after emulsification (Figure 4.11). 


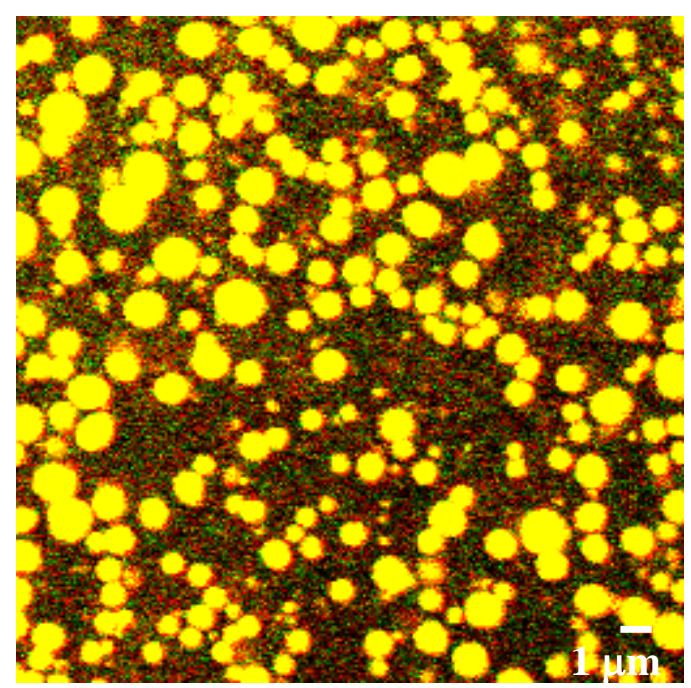

Figure 4.11. 2D confocal microscopy optical cross-section $256 \times 256$ ( $\times 100$ objective, zoom factor 4) of a $15 \mathrm{wt} \%$ toluene emulsion sample prepared by mixing a red fluorescing and a green fluorescing emulsion sample. Snapshot taken within 10 minutes of mixing and 40 minutes after emulsification.

In this third sample, all oil droplets emitted only yellow fluorescence resulting from the mixing of red fluorescing and green fluorescing dye molecules within the droplets. The droplet size distribution of the yellow fluorescing emulsion remained unchanged compared with the parent samples as measured by SLS before and after mixing. Moreover, no phase separation occurred after mixing indicating that the two emulsions were perfectly miscible. Thus, exchange of dye molecules between oil droplets must have occurred with no concurrent droplet growth on a time scale faster than the experimental set up required for observation under the microscope ( $\sim 10 \mathrm{~min})$. Due to the very fast dynamics of the system the initial exchange process when a red fluorescing droplet exchanges with a green fluorescing droplet could not be observed. Such exchange occurs for all oil concentrations and is repeatedly observed.

Four possible mechanisms can be suggested to account for the exchange probed by laser scanning confocal microscopy. The first one is the transfer of dye molecules between oil droplets through the aqueous continuous phase. This mechanism is very unlikely based on first, the low water solubility of the dyes due to their nonpolar chemical structure (Figure 4.6) and second, neither red or green fluorescence is emitted from the continuous phase of the emulsions in Figure 4.7 and Figure 4.10. 
The second possible mechanism is the transport of dye molecules via oil swollen micelles that are present in the continuous phase (DLS, Figure 4.4). Oil transfer via surfactant micelles without any change of the droplet size distribution has been reported in other $\mathrm{O} / \mathrm{W}$ emulsion systems stabilized by nonionic surfactants $[76,77]$.

The third mechanism is the permeation (direct contact) of the oil domains upon droplet collision due to the presence of transient holes in the emulsifier thin film $[78,79]$.

Finally, the fourth potential mechanism is the reversible coalescence of the oil droplets, i.e. the temporary fusion of two or more droplets followed by fission. The postulated mechanism for reversible coalescence proposed by Fletcher et al. for microemulsions [80] involves the formation of a droplet encounter pair with the possibility of molecular permeation through transient holes followed by partial fusion of the interfacial membrane to form a dimer which may or may not be followed by droplet relaxation as illustrated in Figure 4.12.

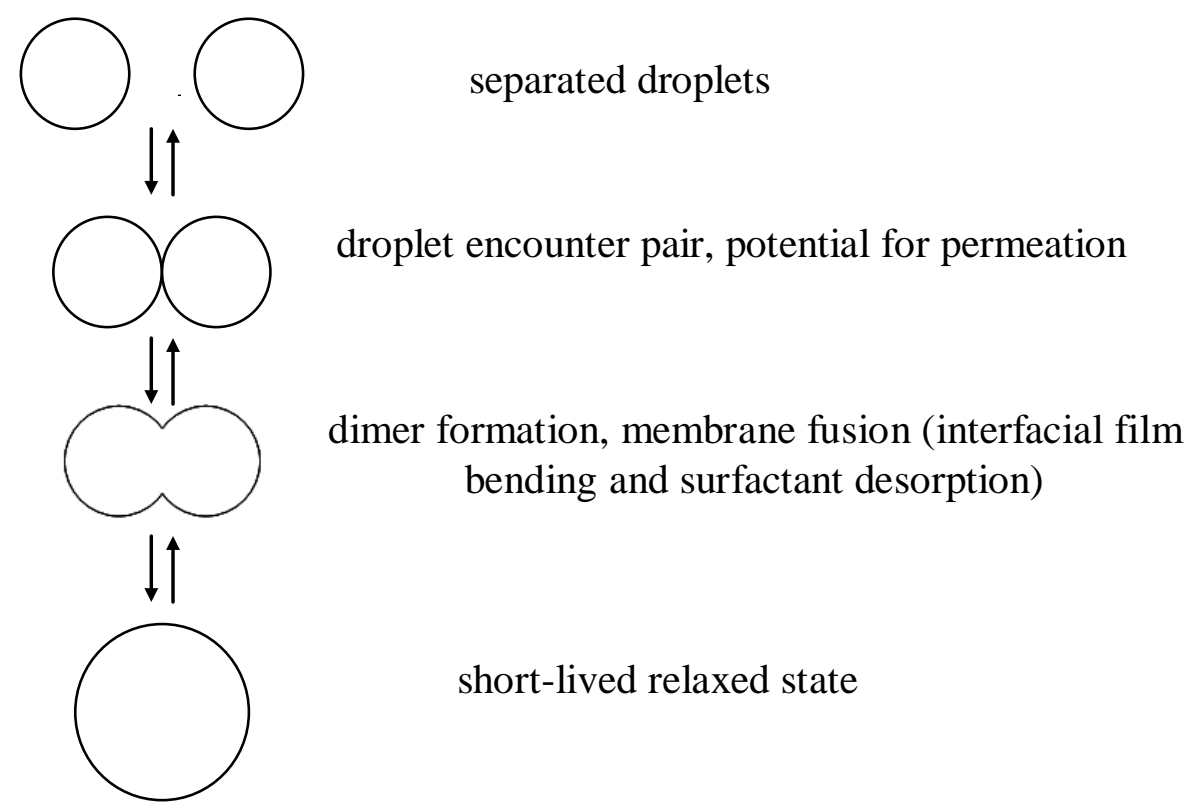

Figure 4.12. Postulated mechanism for the reversible coalescence of droplets.

\subsection{Cryo-SEM}

In order to determine the nature of the oil exchange occurring in toluene/Triton $\mathrm{X}$-100/water emulsions, cryo-SEM was performed, allowing a single time image 
of the internal structure of the emulsions to be obtained (secondary electron detection mode, section 3.5.2). Emulsions were plunged frozen into "slushy" nitrogen at a constant temperature of $-210^{\circ} \mathrm{C}$ about 15 minutes after emulsification such that the internal structure was solidified without changing its inherent state. Freezing was generally achieved within $\sim 1 \mathrm{~s}$ and samples were transferred into the microscope chamber maintaining a temperature of $-130^{\circ} \mathrm{C}$ under high vacuum and low contamination conditions (to avoid water crystals on the sample surface). Access to the internal structure was obtained by fracturing the sample with a cold knife. In Figure 4.13 and Figure 4.14 is shown the internal structure of a freshly emulsified 40 wt\% toluene emulsion upon increasing magnification.

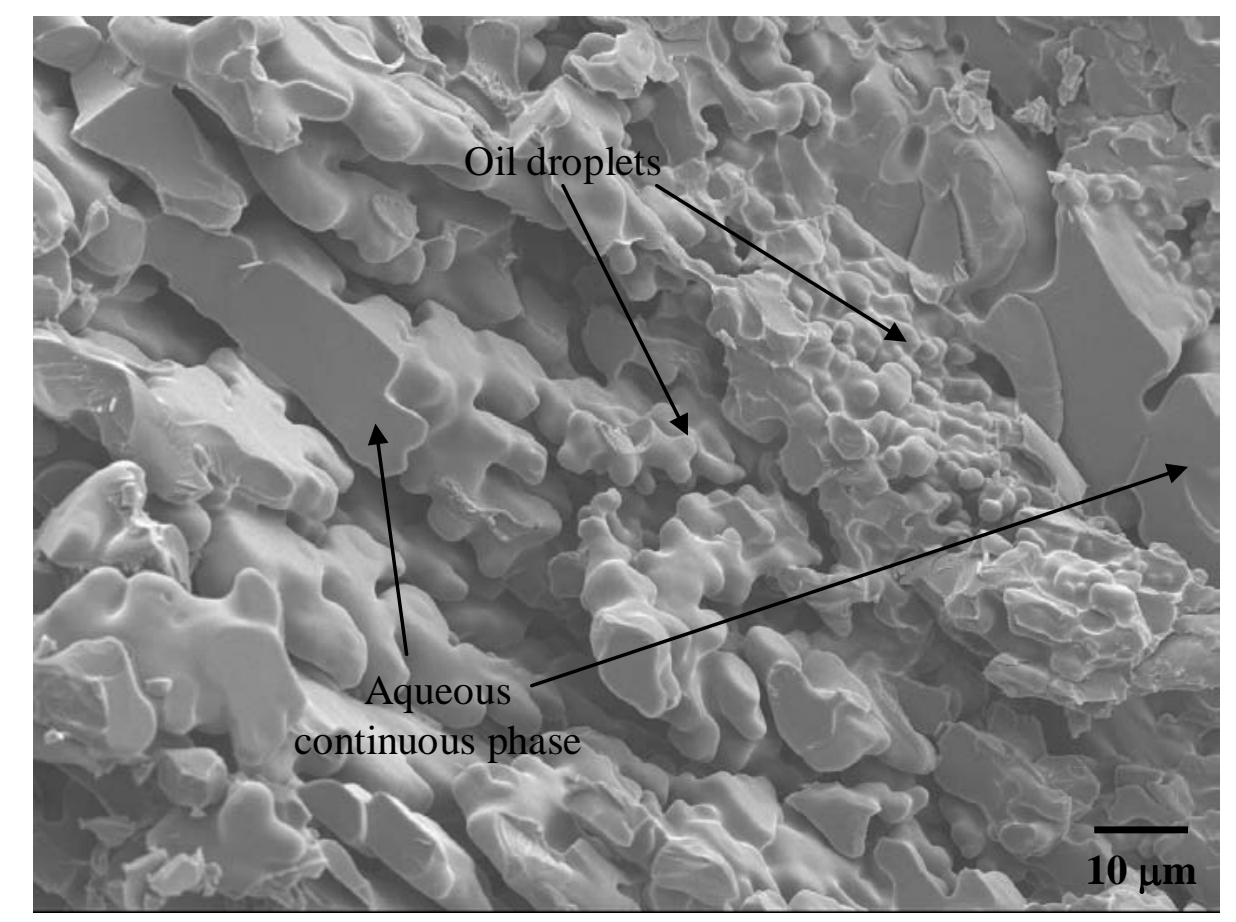

Figure 4.13. Cryo-SEM image of a freshly emulsified $40 \mathrm{wt} \%$ toluene emulsion sample (×1000).

From Figure 4.13, smooth water domains with sharp edges representative of the emulsion's aqueous continuous phase can be distinguished from the dispersed oil droplets. This picture confirms that the internal structure of the emulsion has been preserved during the freezing process and that no melting or crystallisation has occurred. 


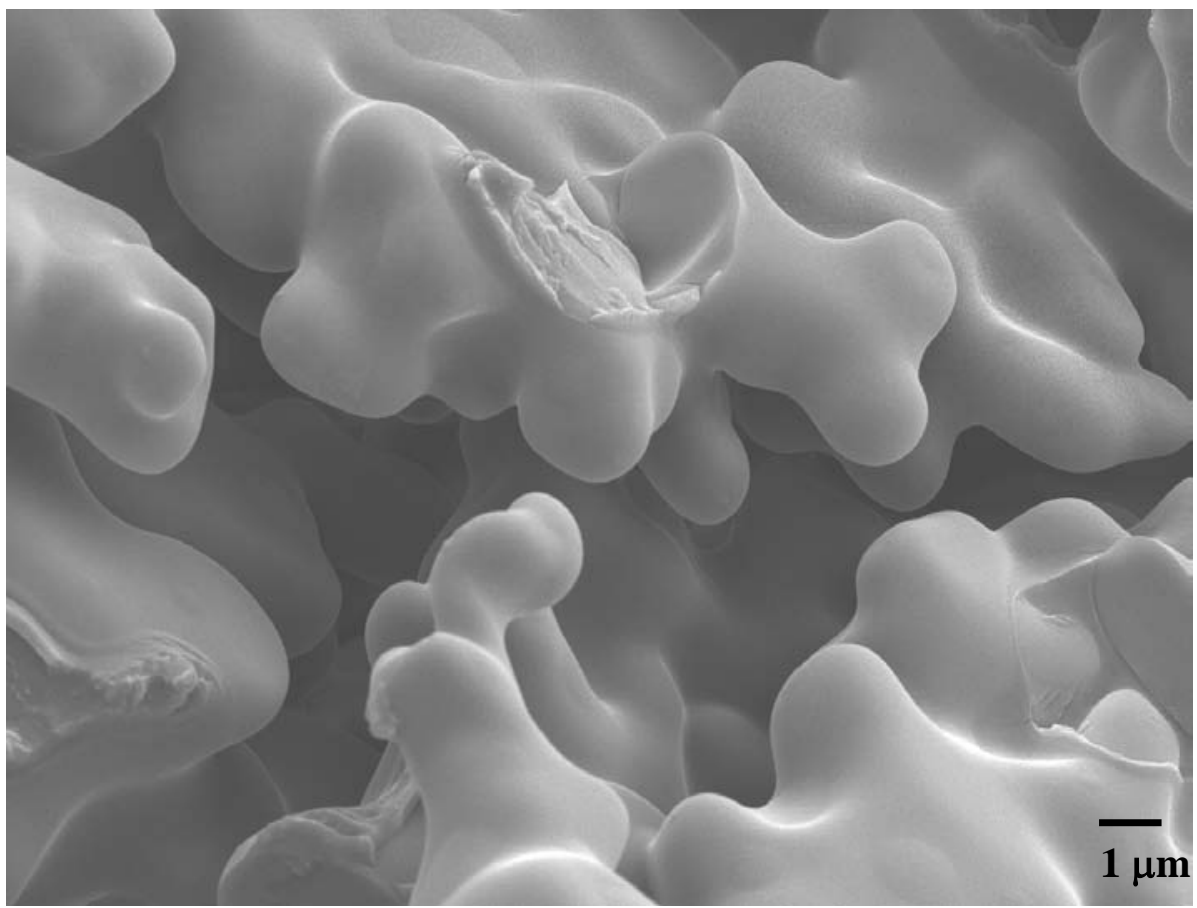

Figure 4.14. Cryo-SEM image of a freshly emulsified $40 \mathrm{wt} \%$ toluene emulsion sample (×5000).

From Figure 4.14, it appears that extensive membrane fusion between oil droplets exists. A network of partially coalesced droplets in very fresh emulsions is formed with no concurrent macroscopic phase separation. The extent of droplet fusion observed in these images therefore does not account for the droplet size distribution measured by SLS (Figure 4.1). The mean droplet diameter measured by SLS for freshly emulsified samples is $0.23 \pm 0.10 \mu \mathrm{m}$ whereas the droplet size distribution based on the cryo-SEM images is much larger with strings of several $1 \mu \mathrm{m}$ diameter droplets being apparent.

Additionally, macroscopic phase separation of a $40 \mathrm{wt} \%$ toluene emulsion sample is initiated $\sim 10$ hours after emulsification. As such it is not possible that normal irreversible coalescence between oil droplets is visualised here only 15 minutes after emulsification since the extent to which fusion is seen here would lead to immediate phase separation. Rather a pathway for the oil exchange process is revealed.

Upon decreasing oil concentration to $15 \mathrm{wt} \%$, a larger proportion of smaller droplets measuring $\sim 0.2 \mu \mathrm{m}$ diameter in addition to larger water domains is observed (Figure 4.15). Significant fusion of the interfacial domain between oil droplets still occurs, as seen at higher magnification (Figure 4.16). 


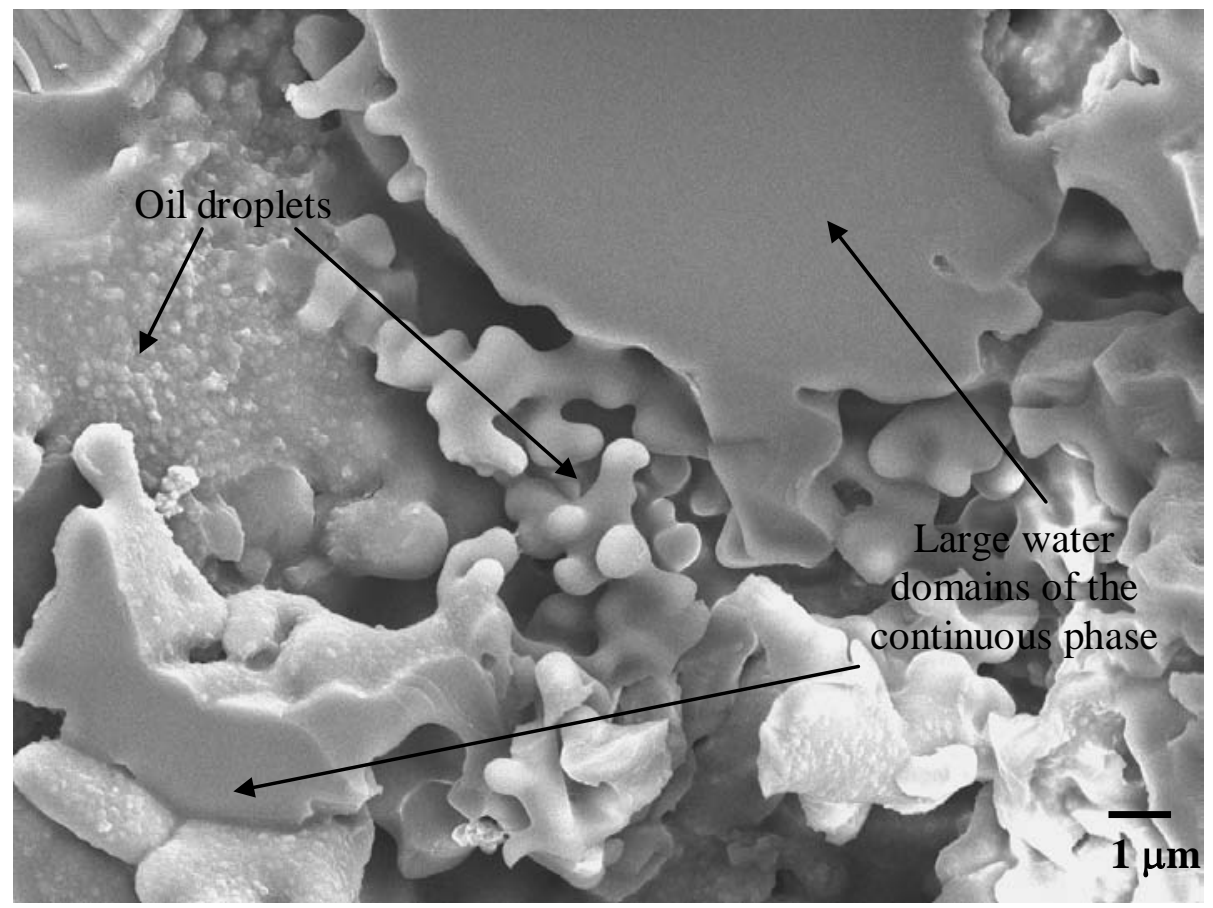

Figure 4.15. Cryo-SEM image of a freshly emulsified $15 \mathrm{wt} \%$ toluene emulsion sample $(\times 5,000)$.

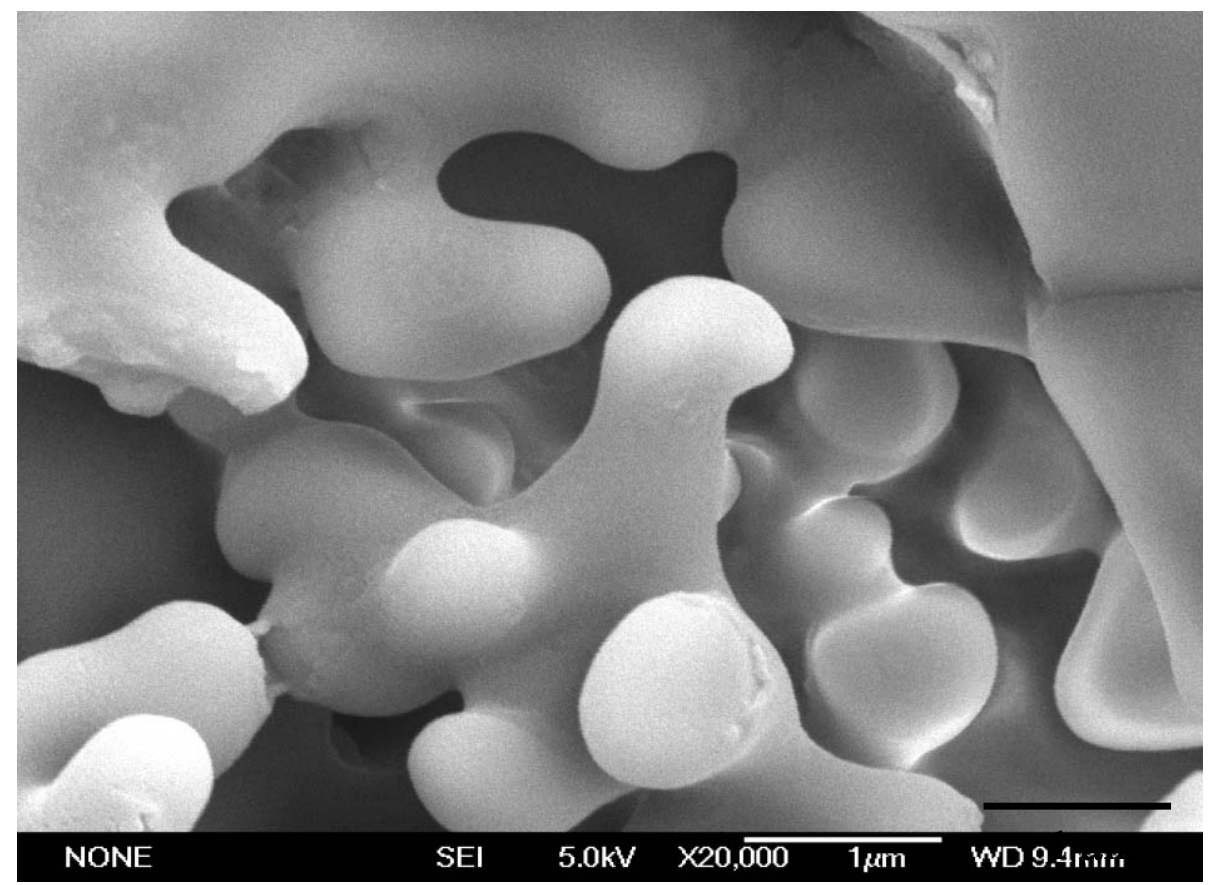

Figure 4.16. Cryo-SEM image of a freshly emulsified $15 \mathrm{wt} \%$ toluene emulsion sample $(\times 20,000)$.

Toluene/Triton X-100/water emulsion samples were difficult to work with using cryo-SEM due to toluene's high vapour pressure and reproducible data devoid of freezing artifacts were obtained only for a few samples. However, $p$-xylene/Triton $\mathrm{X}$-100/water emulsions were easier to work with because $p$-xylene is less volatile 
than toluene. Experimental data using p-xylene instead of toluene including cryoSEM images are presented in chapter 7. Highly reproducible cryo-SEM images were obtained for fresh and aged $p$-xylene emulsion samples.

It is very important to be aware of the possible melting of the oil droplets within the emulsion matrix and adjust the temperature of the microscope stage accordingly. The occurrence of melting was straight forwardly detected. While fracturing a sample that has melted, it is not a solid anymore but rather has a soft texture. The images obtained for a melted $40 \mathrm{wt} \%$ toluene emulsion (Figure 4.17 and Figure 4.18) show little definition and large pools of melted fluid. Even if the melting point of pure oil under high vacuum is higher than the temperature of the microscope stage, it may differ under certain conditions from that of pure oil and become lower within the emulsion matrix. This may lead to misinterpretation of the fusion of droplets in emulsion samples. As a consequence, the microscope stage was held significantly below the emulsion melting point to ensure no melting occurred.

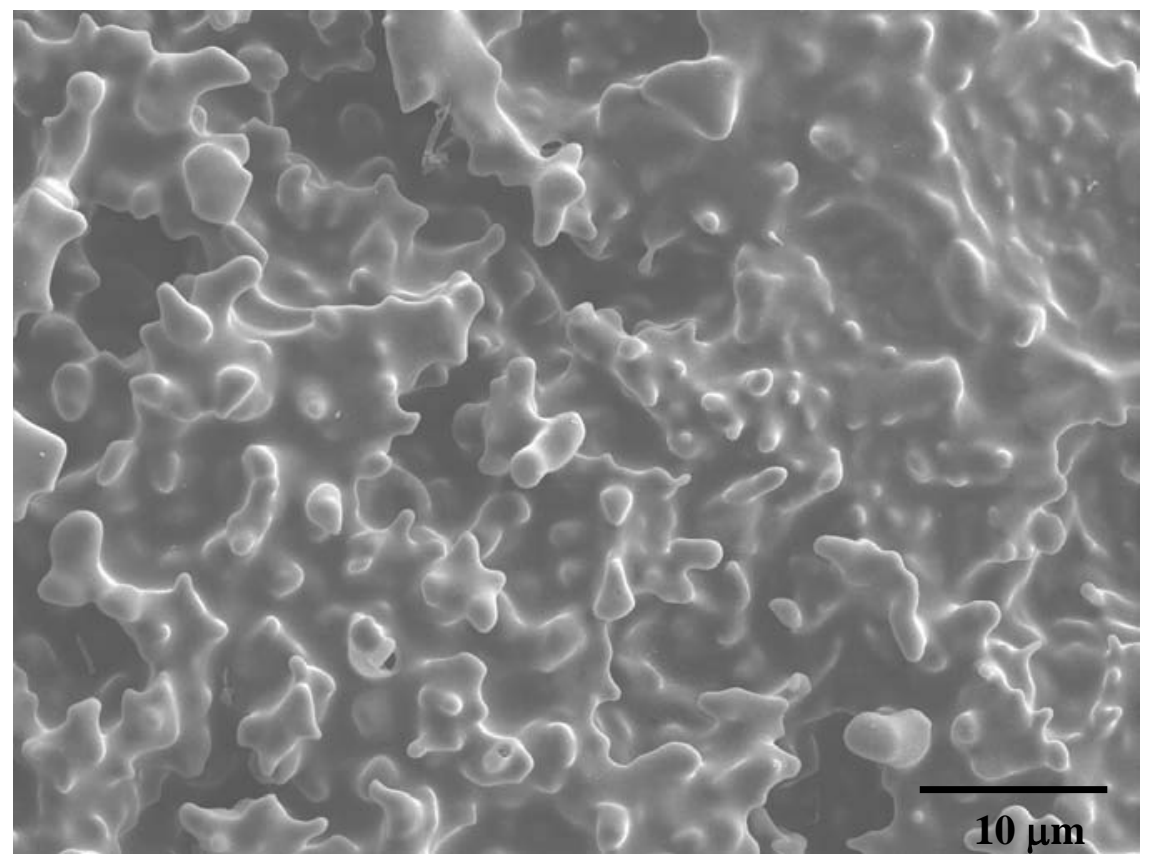

Figure 4.17. Cryo-SEM image of a melted $40 \mathrm{wt} \%$ toluene emulsion $(\times 2,000)$. 


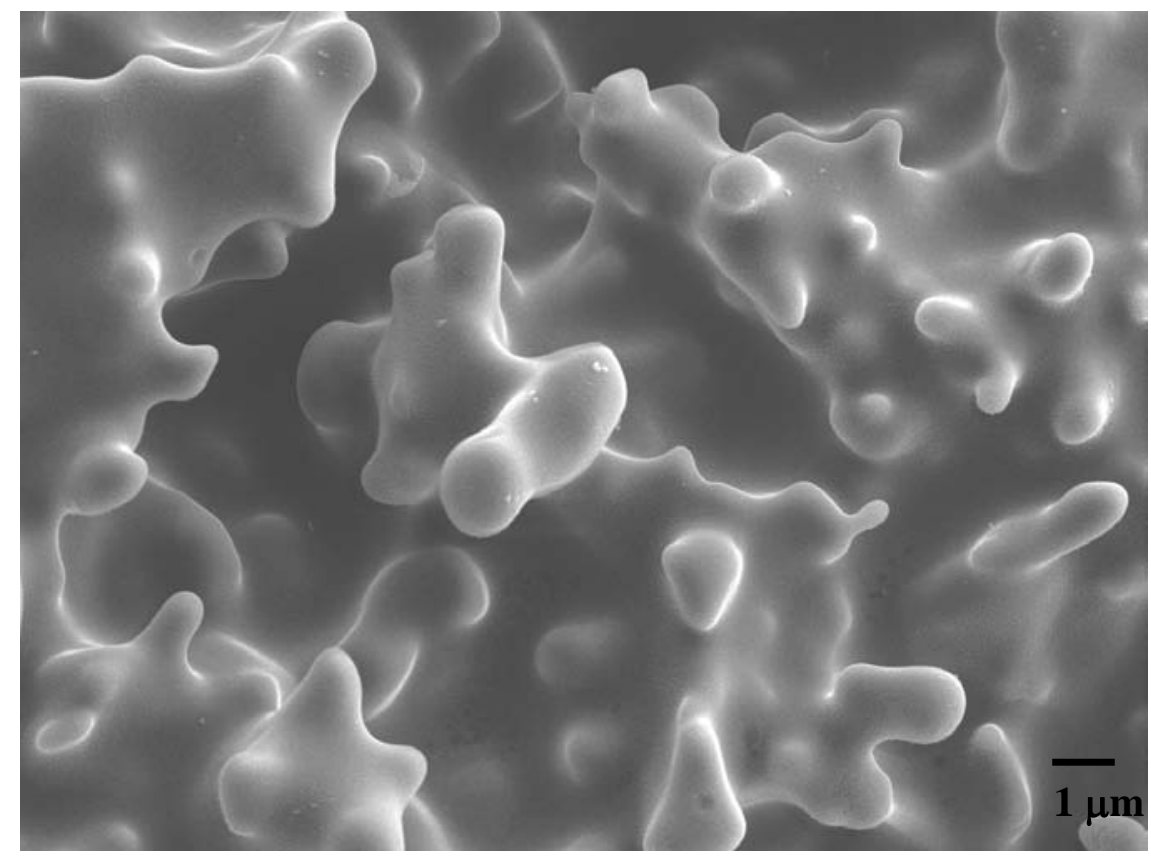

Figure 4.18. Cryo-SEM image of a melted $40 \mathrm{wt} \%$ toluene emulsion $(\times 5,000)$.

Based on the cryo-SEM images, even if transport of oil through the continuous phase or via oil swollen micelles participate to oil exchange between droplets, these two modes of transport are not predominant since droplets significantly interacting with each other via direct contact are observed. However, neither of the remaining two options, i.e. oil permeation upon droplet collision or reversible coalescence via fusion/fission of the droplets can be ruled out at this stage based on the cryo-SEM experiments.

The observed extensive fusion of the interfacial domain between oil droplets suggests that reversible coalescence must be occurring in the system. However it is not possible to clearly distinguish between partially coalesced droplets, encounter pairs driven by coalescence and transient hole pairs. Moreover, oil permeation via transient holes in the interfacial thin film may also act as an exchange agent in addition to reversible coalescence when the droplets form an encounter pair (Figure 4.12).

To complicate things further, observing the fission process in this system was not possible, which would certify the occurrence of reversible coalescence. Also, due to the high polydispersity of the toluene emulsions, the relaxed state of two partially coalesced droplets such as that shown in Figure 4.12 cannot be distinguished from a larger oil droplet already present in the distribution. Hence, for the system investigated here, full reversible coalescence of the droplets as a 
potential mechanism cannot be conclusively stated, as such more correctly the occurrence of reversible coalescence or reversible partial coalescence, resulting in oil transfer, will be considered.

From the confocal and cryo-SEM experiments, it is evident that oil transfer occurs with a time constant that is less than 10 minutes and most likely less than a few seconds based on the real time confocal data. However neither of these techniques offer a sufficiently short experimental time scale to monitor such fast dynamics.

\subsection{PFG-NMR}

The dynamics of the oil phase were probed by PFG-NMR using a PGSTE sequence (

Figure 3.11) as described in section 3.4.3.3. The NMR echo attenuation of the oil signal as a function of gradient strength for a polydisperse emulsion is a multiexponential decay given by Eq. 3.12 for all values of the experimental observation time, $\Delta$. Fitting the multiexponential signal yields a distribution of diffusion coefficients mapping the underlying distribution of droplet sizes since the diffusion coefficient is closely related to droplet size (Eq. 3.4). Hence, for toluene/Trion X-100/water emulsions investigated here it is expected that the raw attenuation signal would follow a multiexponential decay mirroring the polydispersity of the samples as measured by SLS (Figure 4.1). However, the echo attenuation of the oil signal, above a given observation time, which is oil concentration dependent, and up to $3 \mathrm{~s}$, follows a single exponential decay (straight line) as a function of gradient strength (Eq. 3.13). In Figure 4.19 is shown the representative diffusion echo attenuation of the oil signal for freshly emulsified samples prepared with toluene between 5 and 55 wt\%. 


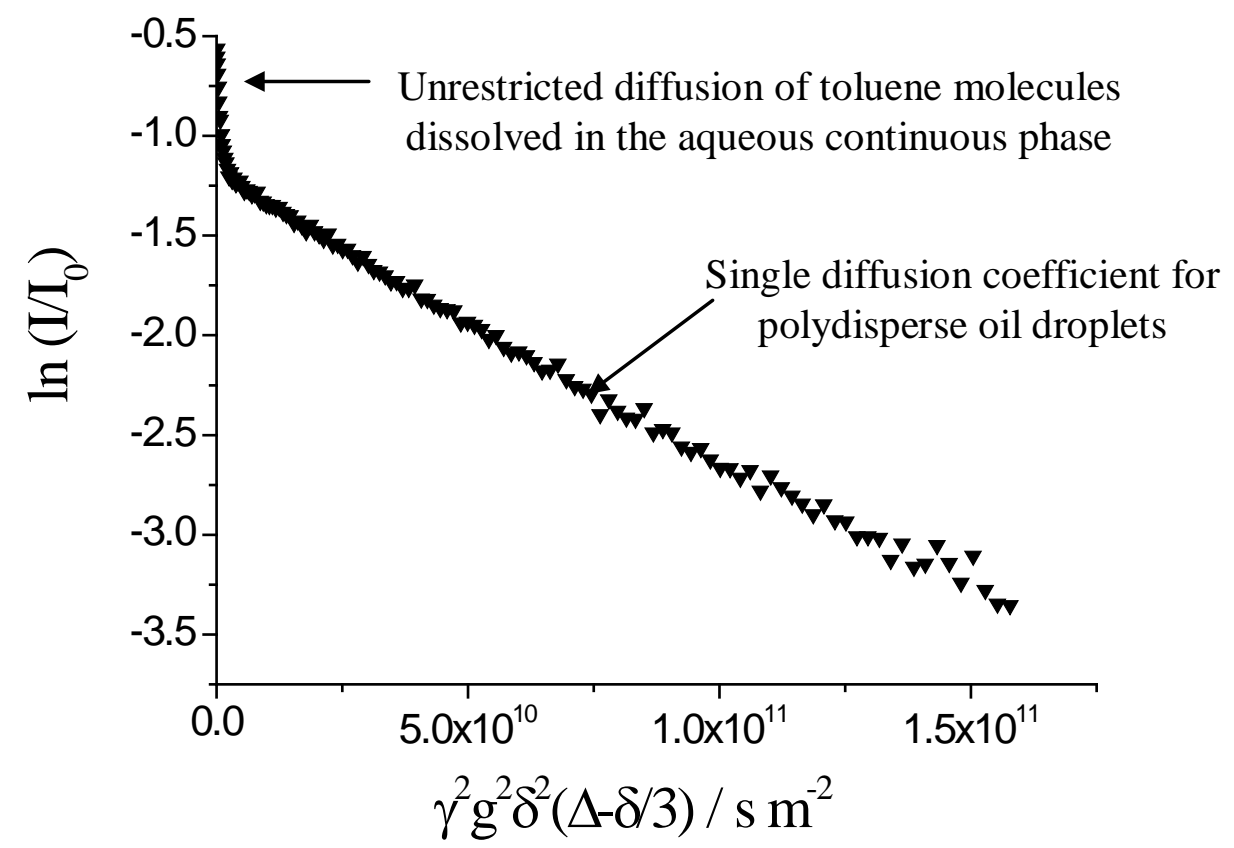

Figure 4.19. Representative diffusion echo attenuation of the oil signal of emulsions freshly prepared between 5 and $55 \mathrm{wt} \%$ toluene, here $40 \mathrm{wt} \%$. The observation time, $\Delta$, spans from $300 \mathrm{~ms}$ to 3 s depending on oil concentration.

From Figure 4.19, it is evident that two domains are observed. The first, a fast diffusing domain, observed at low gradient strength, correlates to a diffusion coefficient in the range of $1.0 \times 10^{-9}$ and $2.3 \times 10^{-9} \mathrm{~m}^{2} \mathrm{~s}^{-1}$ depending on the volume fraction of the continuous phase. Toluene is partially soluble in water (0.56 $\mathrm{g} / \mathrm{L}$ at $298 \mathrm{~K} \mathrm{[81])} \mathrm{hence} \mathrm{it} \mathrm{is} \mathrm{to} \mathrm{be} \mathrm{expected} \mathrm{that} \mathrm{a} \mathrm{small} \mathrm{fraction} \mathrm{of} \mathrm{the}$ toluene will be present in the continuous phase. The self-diffusion coefficient at $298 \mathrm{~K}$ for pure toluene is $D=2.24 \times 10^{-9} \mathrm{~m}^{2} \mathrm{~s}^{-1}$ so this fast diffusing domain corresponds to unrestricted diffusion of toluene molecules dissolved directly in the aqueous continuous phase. This fast component is also measured when toluene is dissolved directly in water without Triton X-100 confirming that toluene is partially soluble in water. By replacing water with $\mathrm{D}_{2} \mathrm{O}$, in which toluene is not soluble, this fast diffusing contribution is removed (Figure 4.20). 


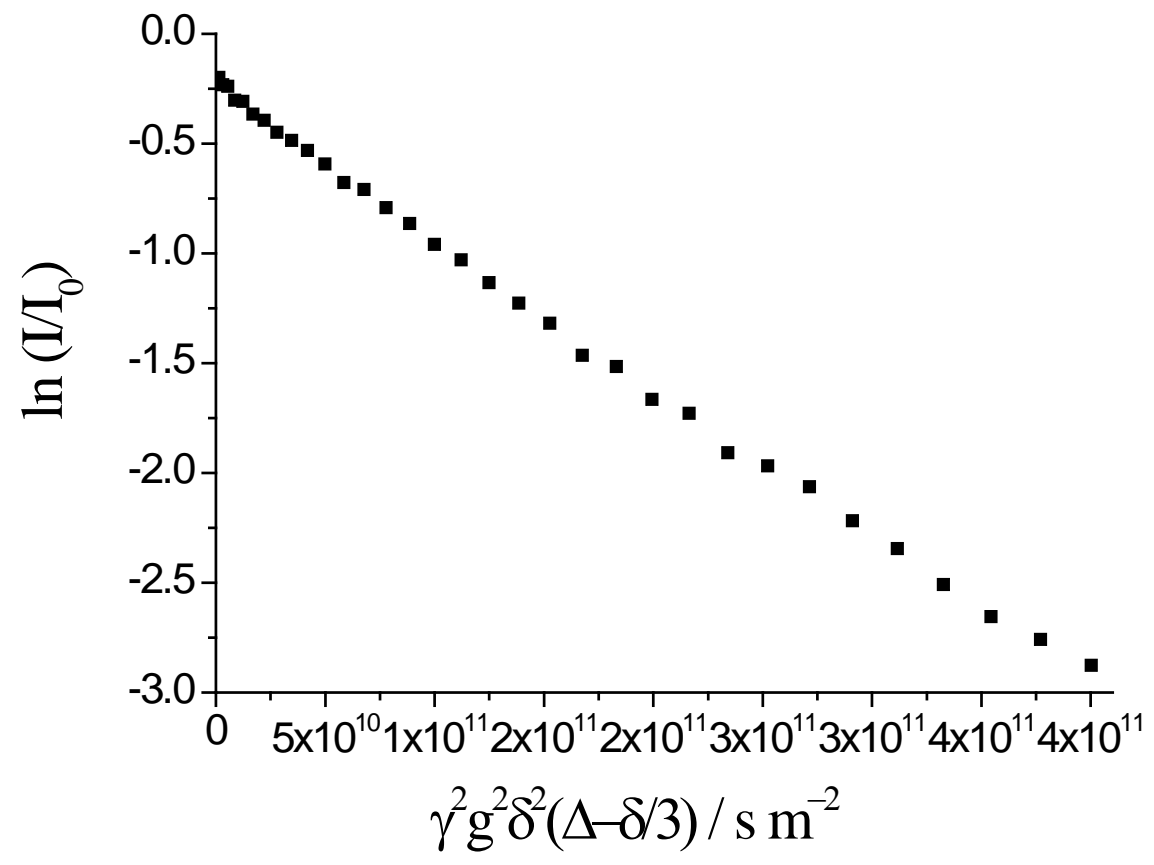

Figure 4.20. Representative diffusion echo attenuation of the oil signal of emulsions freshly prepared between 5 and 55 wt \% toluene, here 15 wt\%, by replacing water with $D_{2} O$.

With the fast component accounted for, the slow diffusing domain arises from diffusion of oil in droplets and oil swollen micelles and diffusion of droplets and oil swollen micelles themselves. Due to the polydispersity of the droplets and oil swollen micelles these different motions should result in a distribution of diffusion coefficients for all observation times, $\Delta$, and hence a multiexponential decay of the echo attenuation as a function of gradient strength. However, the slow diffusing domain is a single exponential decay with a mean diffusion coefficient $D=(1.5 \pm 0.1) \times 10^{-11} \mathrm{~m}^{2} \mathrm{~s}^{-1}$ (Figure 4.19) in the range $5-55 \mathrm{wt} \%$ toluene. The observed single exponential decay as a function of the gradient strength does not account for the polydispersity of toluene/Triton X-100/water emulsion droplets. Above a given NMR observation time, the system behaves as if all oil molecules experience the same environment and travel the same distance over the same duration; that is, motional averaging of the oil molecules is measured as opposed to their true diffusion, resulting in a single exponential decay. The motional averaging, a result of oil exchange between the droplets, occurs on a time scale that is faster than the observation time. The analysis of this time scale as a function of oil concentration will be presented in the next chapter. 


\subsection{Discussion}

The transfer of dispersed phase or constituents solubilised in the dispersed phase between emulsion droplets in highly concentrated W/O emulsions has already been monitored using PFG-NMR [48, 62, 82, 83]. In these systems, water molecules or water soluble constituents are able to migrate from one droplet to another by crossing the permeable interfacial membrane and diffusing through the oily continuous phase. Hence, the echo attenuation is a single exponential decay as a function of the gradient strength over the entire range of observation times, $\Delta$, e.g. from $10 \mathrm{~ms}$ to $1 \mathrm{~s}$ in the study of Malmborg et al. [82]. The diffusion coefficient associated with the single exponential decay of the echo attenuation of the water signal in Balinov et al.'s experimental work [48], is an order of magnitude slower as compared with that of bulk water due to obstruction effects of the surfactant films that act as barriers. Using a simulation procedure they could calculate a permeability coefficient for the water crossing the interfacial domain. However, this mode of transfer between "leaky" emulsion droplets requires the solubility of the exchanged constituents in the continuous phase [82], which does not account for our confocal microscopy data in which water insoluble dyes are exchanged between oil droplets. Additionally, the direct contact of "leaky" droplets for exchange to occur is not necessary since the dispersed phase is able to reside in the water, emulsifier and oil domains. This is in contradiction with the extensive droplet contact observed in cryo-SEM micrographs.

On the other hand, oil exchange aided by surfactant micelles that are present in the continuous phase has been commonly probed in the destabilisation of alkane/nonionic surfactant/water emulsions upon the occurrence of enhanced Ostwald ripening. Micelles carry alkane molecules from smaller to larger droplets leading to droplet growth, the rate of which increases upon decreasing the length of the hydrocarbon chain $[49,84,85]$. Because the droplet size distribution of our emulsion system remains unchanged during the oil exchange as measured by SLS, the exchange does not pertain to emulsion destabilisaton but rather occurs independently of coalescence and/or Ostwald ripening that both lead to droplet growth. 
Furthermore, our NMR data follow a single exponential decay. In standard or enhanced Ostwald ripening the distribution of oil droplet sizes would be evident as a multiexponential decay, since the mechanism involves the oil swollen micelles in combination with the oil droplets and not the oil droplets directly interacting with each other.

Some examples of oil exchange between emulsion droplets via surfactant micelles with no simultaneous droplet growth have been reported in the literature. McClements et al. [77] followed the droplet size and concentration of nhexadecane droplets stabilised by a nonionic surfactant over time using light scattering and turbidity techniques. The droplet concentration decreased as a function of time with no modification of the droplet size distribution, hence they considered a dynamic equilibrium between oil solubilised in emulsion droplets and that in surfactant micelles, but the driving force for such an exchange remains unknown.

Several factors affect the rate of oil exchange in emulsions via surfactant micelles, such as the surfactant concentration in the aqueous continuous phase [86] or the initial droplet size and concentration [87]. Moreover, McClements and coworkers [76] probed, by differential scanning calorimetry, the exchange of pure nhexadecane and pure octadecane droplets after mixing the two emulsions with no concurrent change in the droplet size distribution. The rate of exchange increased with surfactant concentration in the continuous phase indicating that micelles were involved in oil transfer. They proposed that entropy is the predominant contribution for exchange of n-hexadecane and octadecane molecules between droplets to occur since a system in which oil is evenly distributed in all the droplets is more entropically favorable than one containing a mixture of different sized pure oil droplets. However, surfactant micelles cannot be considered as the predominant mode of transport for oil exchange in toluene/Triton X-100/water emulsions since in addition to the extensive droplet fusion perceived in cryoSEM, droplets must come into direct contact for complete motional averaging to be detected in PFG-NMR. As such, based on the three different experimental techniques, it is proposed that oil permeation on droplet collision and partial reversible coalescence of the droplets are the only plausible mechanisms for the observed oil exchange. 
Transient holes are present at the surface of emulsion droplets as a result of thermal fluctuations of the interfacial surfactant film and their positions vary on the surface of the droplets [78, 79, 88]. Taisne and Cabane [89] proposed molecular permeation in an $\mathrm{O} / \mathrm{W}$ emulsion stabilised by a nonionic surfactant as an oil transfer mechanism to account for the transport of oil from smaller to larger alkane droplets. They affirmed that Ostwald ripening cannot explain droplet growth in this system since no change in the rate of oil exchange was measured upon variation of the oil solubility in the aqueous continuous phase (tetradecane and hexadecane gave identical results although the former has a solubility in water an order of magnitude larger than the latter). On the contrary, temperature and addition of an ionic surfactant had a significant effect on the exchange rate. A temperature rise increases the efficiency of molecular permeation by surmounting the activation energy and also modifies the structure of the surfactant film. Addition of an ionic surfactant decreases the rate of the permeation process by acting as a physical barrier against direct droplet contact. Such exchange results in droplet growth and emulsion destabilization.

Furthermore, Schmitt et al. [90] studied the coarsening of alkane-in-water emulsions stabilised by nonionic poly(oxyethylene) surfactants. They found that the rate of Ostwald ripening in the presence of oil swollen micelles did not vary with surfactant concentration in the continuous phase and considered a permeation-controlled Ostwald ripening as the main destabilisation process.

Nonetheless, an oil exchange via molecular permeation upon droplet collision that does not lead to emulsion destabilisation (droplet growth) has not been reported in the literature to date. The driving force that would lead to such an exchange is unknown.

When considering possible mechanisms for the oil exchange, the extensive membrane fusion seen in the cryo-SEM images indicates that reversible coalescence or partial coalescence of the oil droplets should be considered as the second potential mechanism for oil exchange in the system studied in this project, although fission of the droplets has not been observed experimentally.

In microemulsions, the equilibrium or spontaneous curvature of the surfactant film is determined by the most efficient packing of the surfactant molecules at the oil/water interface based on the surfactant chemical structure matched with the geometry, as well as the system temperature, composition, salt concentration etc 
[19]. The equilibrium curvature of the surfactant film defines an indefinitely stable size distribution in microemulsion systems, as such fission of the droplets is required once fusion has been initiated.

The dynamic equilibrium of reversible coalescence occurring in thermodynamically stable microemulsions is poorly understood. However it is known that the reduction of the total surface area, through droplet fusion facilitated by attractive forces (van der Waals, solvation and hydrophobic forces), competes with the minimization of entropy and steric hindrance associated with the formation of larger droplets [19]. Reversible coalescence of droplets has been experimentally evidenced in both W/O and O/W microemulsions ( $\sim 5-30 \mathrm{~nm}$ diameter). Fletcher et al. [80] identified this process by monitoring fast chemical reactions between reactants confined within the droplets of two separated W/O microemulsions stabilised by an ionic surfactant. The fusion-fission takes place on a millisecond time scale. Clark et al. $[80,91]$ used time-resolved fluorescence to measure the fluorescence intensity decay of both $\mathrm{W} / \mathrm{O}$ and $\mathrm{O} / \mathrm{W}$ microemulsions where a fluorescent molecule and a quencher were confined within separated droplets. Several workers probed the influence of the surfactant rigidity, temperature and oil chain length on the rate of reversible coalescence [80, 92-94].

To date, the occurrence of reversible coalescence or partial coalescence in kinetically stabilised systems such as emulsions has not been reported. The thermodynamically stable state of emulsions is the phase separation into two macroscopically isolated oil and water domains. As such, once fusion of the interfacial membrane is initiated, a repulsive force is required for fission to occur in these systems. Such a force is not predicted to exist based on the DLVO theory (section 2.3). However, the study of Burschka and coworkers [95] does allude to the possibility of realising reversible coalescence in a kinetically stabilized system even if a direct correlation between their theoretical investigation in one dimension and the polydisperse kinetically controlled oil/Triton X-100/water systems cannot be made. They solved the diffusion-limited reversible one-species coagulation in one dimension using a diffusion-reaction equation to describe the local density. As opposed to the DLVO theory, they considered kinetic effects based on diffusion events and the strength of the interaction between colliding 
bodies. Such a description is valid for either an equilibrium system where the equilibrium state is a state of maximal entropy or a non-equilibrium steady-state system for which the probability of colliding particles is small. Oil/Triton X100/water emulsion systems are non-equilibrium steady-state systems, however for all oil concentrations investigated, the frequency of droplet collision is significant, hence this theory cannot be directly applied to the oil/Triton $\mathrm{X}$ 100/water emulsions.

Additionally, several experimental results published over the past decades, such as the existence of structural forces [23-25], also point to limitations in our current knowledge of colloidal stability based on the DLVO theory.

\subsection{Conclusion}

In this chapter the microstructure of toluene/Triton X-100/water emulsions was described upon varying oil concentration from 5 to $70 \mathrm{wt} \%$. The droplet size distribution (35 $\mathrm{nm}$ to $3 \mu \mathrm{m}$ diameter) is independent of oil content between 5 and $55 \mathrm{wt} \%$ whereas an increase of the mean droplet size is observed for higher oil concentrations between 60 and 70 wt\%. Furthermore, oil swollen micelles (9-30 nm diameter) are distributed in the continuous phase of the emulsions for all toluene concentrations.

On the other hand, the occurrence of oil exchange between emulsion droplets with no concurrent droplet growth via an oil permeation upon droplet collision or a reversible coalescence mechanism or a combination of the two was evidenced using laser scanning confocal microscopy, cryo-SEM and PFG-NMR. The exchange of water insoluble hydrophobic dye molecules between oil droplets evidenced by confocal microscopy indicates that the transport of oil through the continuous phase is not the predominant mechanism for oil exchange. The cryoSEM experiments indicate that oil exchange occurs via preponderant droplet contact, the extent of which is too important to be due to normal coalescence given the emulsion's macroscopic stability and the absence of droplet growth. In PFG-NMR experiments, a motional averaging of the oil domain arising from oil exchange was detected for long observation times. The motional averaging of the oil, superposed to the cryo-SEM data, suggests that oil transfer via surfactant 
micelles does not significantly participate to oil exchange. In conclusion, oil permeation upon droplet collision and/or reversible coalescence (or reversible partial coalescence) of the droplets generate oil exchange between toluene droplets in toluene/Triton X-100/water emulsions. In either case, oil exchange does not induce droplet growth, hence is not linked to emulsion destabilisation processes. 


\section{Chapter 5 Kinetics of oil exchange}

\subsection{Introduction}

The determination of the kinetics of the oil exchange occurring in emulsion systems are of fundamental importance in order to gain more understanding of the driving forces involved in the exchange process. Additionally, the investigation of the different factors that may affect the time scale of oil exchange is essential to be able to predict and manipulate the kinetics of exchange.

In this chapter is reported the determination of the time scale of oil exchange occurring between oil droplets of toluene/Triton X-100/water emulsions using PFG-NMR techniques. The NMR observation time, $\Delta_{\text {switch, defines the time }}$ constant of the oil exchange. This observation time corresponds to the time at which there is a switch between a multiexponential decay, where individual droplet and oil molecule motions are probed, and a single exponential decay of the echo attenuation as a function of gradient strength, where motional averaging of the oil diffusion is measured.

Based on the analysis of the diffusion spectra extracted from the multiexponential decay attenuation data using an inverse Laplace transform and the light scattering data, information was gained on the microstructure of the system as a function of oil concentration.

The kinetics of the oil exchange process is strongly influenced by the increase of the mean droplet size above $60 \mathrm{wt} \%$ oil and the toluene content between 5 and 55 wt\% defining the droplet concentration and controlling the properties of the interfacial domain such as the surfactant packing at the oil/water interface. The presence of a background electrolyte solubilised in the continuous phase further alters the rate of oil exchange through the formation of a steric repulsive barrier. 


\subsection{Kinetics of oil exchange}

The rapid oil exchange occurring in toluene/Triton X-100/water emulsions was both monitored and quantitatively determined using a PGSTE experiment (section 3.4.3.3) upon varying the NMR observation time, $\Delta$, between $6.3 \mathrm{~ms}$ and $3 \mathrm{~s}$ (available observation window, the short limit depends on the pulse sequence parameters and the long on the sample relaxation constants). Note that both cryoSEM and laser scanning confocal microscopy techniques could not probe the time scale of such a rapid process.

In chapter 4 , it was indicated that beyond a certain observation time, $\Delta$, which is oil concentration dependent, the echo attenuation of the oil signal follows a single exponential decay as a function of the gradient strength. Increasing $\Delta$ above this value did not make any difference in the attenuation data obtained, i.e. the echo attenuation followed a single exponential decay with a constant slope equal to $D$ $=(1.5 \pm 0.1) \times 10^{-11} \mathrm{~m}^{2} \mathrm{~s}^{-1}$ (Figure 4.19). However upon decreasing $\Delta$ below this specific observation time value, a switch between a single exponential and a multiexponential decay of the echo attenuation occurred. Hence this specific observation time was termed $\Delta_{\text {switch }}$ (Figure 5.1). 


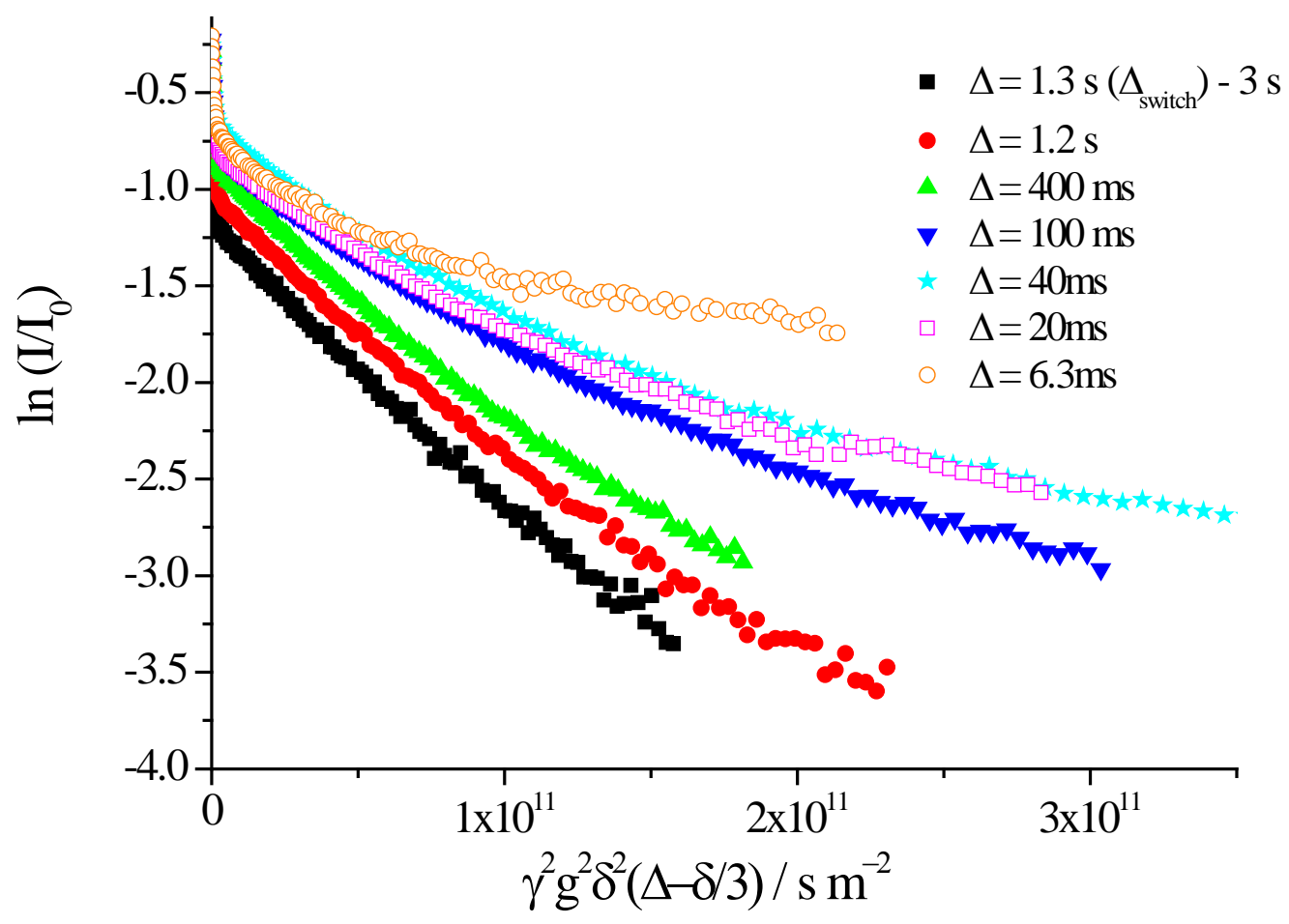

Figure 5.1. Diffusion echo attenuation of the oil signal for a fresh $40 \mathrm{wt} \%$ toluene emulsion. A single exponential decay is observed for an observation time, $\Delta$, between $1.3 \mathrm{~s}\left(\Delta_{\text {switch }}\right)$ and $3 \mathrm{~s}$ whereas a multiexponenttial decay is measured for $\Delta$ between $6.3 \mathrm{~ms}$ and $1.2 \mathrm{~s}$. These echo attenuations are representative of emulsion samples between 5 and $\sim 55 \mathrm{wt} \%$ toluene.

In Figure 5.1 is shown the echo attenuation of the oil signal as a function of the gradient strength for a freshly emulsified $40 \mathrm{wt} \%$ toluene sample upon decreasing the observation time, $\Delta$. The behaviour of the echo attenuation upon variation of $\Delta$ for a 40 wt $\%$ toluene sample is representative of the concentration range between 5 and $\sim 55$ wt\% oil although the values of $\Delta$ for which transitions occur are concentration dependent and will be detailed in section 5.3. Here, focus is made on a $40 \mathrm{wt} \%$ toluene emulsion to illustrate the analysis of the different transitions. The echo attenuation of the oil signal follows a single exponential decay as a function of the gradient strength with a diffusion coefficient $D=(1.5 \pm$ $0.3) \times 10^{-11} \mathrm{~m}^{2} \mathrm{~s}^{-1}$ for $\Delta$ comprised between 1.3 and 3 s, i.e. $\Delta_{\text {switch }}=1.3 \mathrm{~s}$ (Figure 5.1). This single exponential decay corresponds to the motional averaging of the oil diffusion which results from oil exchange between emulsion droplets on a time scale faster than $\Delta_{\text {switch. }}$ Upon decreasing $\Delta$ to $1.2 \mathrm{~s}$, the polydisperse nature of the emulsion starts to be revealed since the echo attenuation becomes multiexponential. However, only a portion of the individual droplet motions is probed for any given value of $\Delta$. By further decreasing $\Delta$, the echo attenuation 
becomes more and more multiexponential, hence more individual droplet motions are detected.

The distribution of diffusion coefficients related to individual droplet and oil molecule motions can be extracted from the multiexponential decay signal using an inverse Laplace transform (section 3.4.3.5). A nonnegative least-squares fit weighted by an additional regularisation function is applied to the attenuation data giving a diffusion spectrum (Figure 5.2).

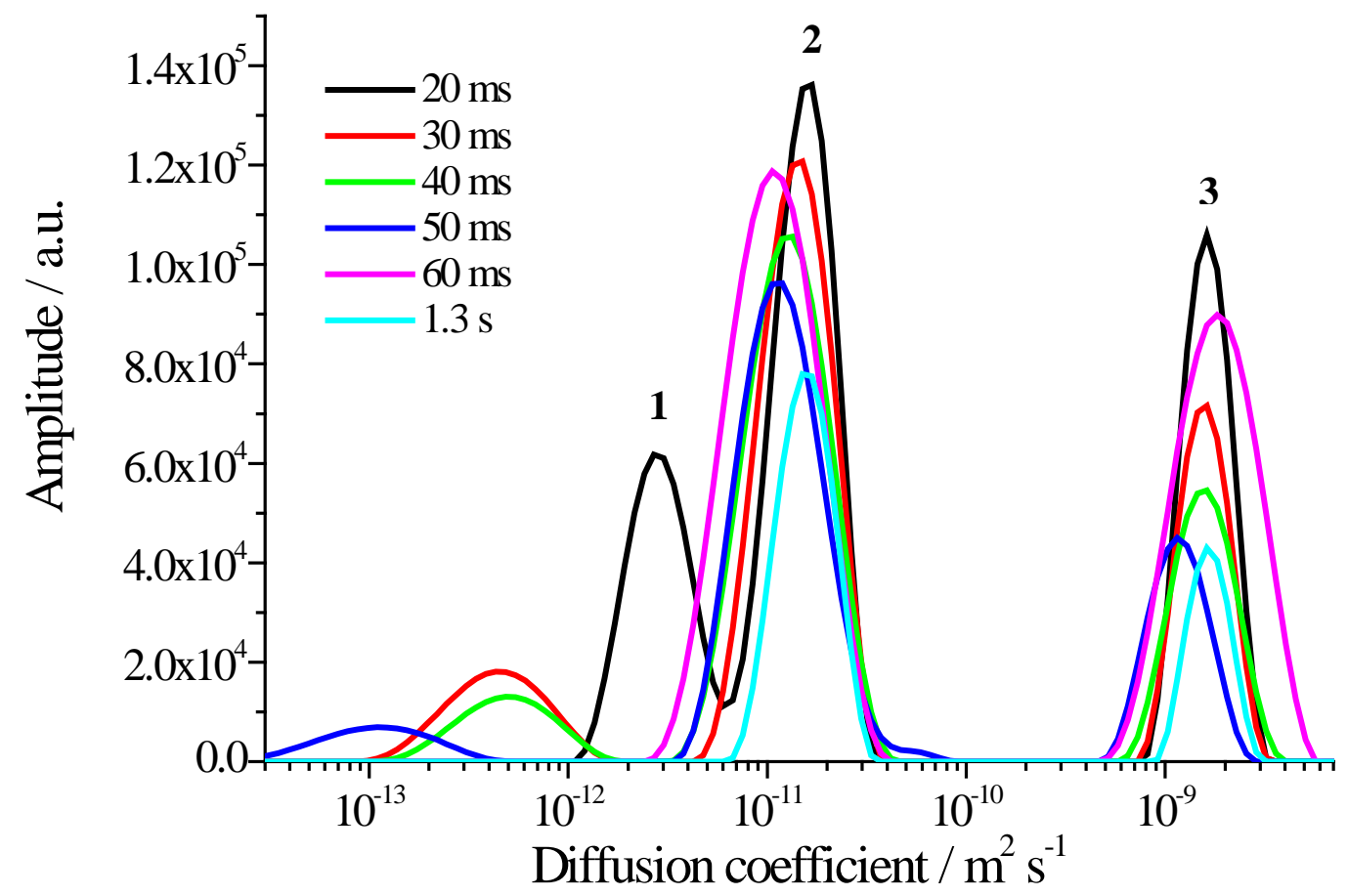

Figure 5.2. Representative diffusion spectra of toluene molecules and droplets, determined at various experimental observation times $\left(\Delta=20,30,40,50\right.$ and $60 \mathrm{~ms}$, all below $\left.\Delta_{\text {switch }}=1.3 \mathrm{~s}\right)$ for emulsions prepared with $40 \mathrm{wt} \%$ toluene. At short $\Delta$ values, three peaks numbered 1,2 and 3 may be distinguished.

The motion of oil molecules inside droplets may be restricted by droplet size while the movement of the droplets themselves depend on both the droplet size and the mean interdroplet distance. As such, for a given observation time, $\Delta$, both restricted and unrestricted diffusion can be measured correlating to the motions of only a portion of the sample. Thus, the different droplet sizes and spacing between the droplets may be probed upon varying the observation time, $\Delta$.

The diffusion spectra of toluene molecules and droplets in a fresh $40 \mathrm{wt} \%$ toluene sample obtained for different $\Delta$ values are shown in Figure 5.2. The distribution of diffusion coefficients can be associated with the unrestricted and/or restricted 
diffusion of droplets and oil swollen micelles as well as the unrestricted and/or restricted diffusion of oil molecules within droplets and oil swollen micelles based on the droplet and oil swollen micelle size distributions (Figure 4.1 and Figure 4.4) coupled with the dependence of the diffusion spectrum on the observation time, $\Delta[96]$.

The peak corresponding to the fastest diffusion (number 3, Figure 5.2) centered at $\sim 1.6 \times 10^{-9} \mathrm{~m}^{2} \mathrm{~s}^{-1}$ for all values of $\Delta$ does not correspond to the motion of the dispersed phase but is associated with the unrestricted diffusion of toluene molecules dissolved directly in the aqueous continuous phase as discussed earlier. The remaining peaks may be associated with eight possible motions: 1) unrestricted diffusion of oil droplets, 2) unrestricted diffusion of oil swollen micelles, 3) unrestricted diffusion of oil molecules within droplets, 4) unrestricted diffusion of oil molecules within oil swollen micelles, 5) restricted diffusion of droplets, 6) restricted diffusion of oil swollen micelles, 7) restricted diffusion of oil molecules within droplets and 8) restricted diffusion of oil molecules within oil swollen micelles.

Motions 3) and 4) cannot be observed experimentally in fresh samples because the observation times required to observe unrestricted diffusion of toluene molecules with a known diffusion coefficient, $D=2.24 \times 10^{-9} \mathrm{~m}^{2} \mathrm{~s}^{-1}$, confined within oil swollen micelles and droplets of $9 \mathrm{~nm}$ to $3 \mu \mathrm{m}$ diameter is in the range of 2 ns to $200 \mu$ s (Eq. 3.15). Such short observation times are 30 times to $3 \times 10^{6}$ times faster than the lower available limit of the instrument, i.e. $\Delta=6.3 \mathrm{~ms}$.

Furthermore, between 5 and $\sim 55 \mathrm{wt} \%$ toluene, the average small size of the oil swollen micelles and droplets also precludes restricted diffusion of oil molecules within oil swollen micelles and droplets being observed when coupling the sizes of the droplets with the observation times available and the lower measurable limit of the diffusion coefficients $\left(\sim 10^{-14} \mathrm{~m}^{2} \mathrm{~s}^{-1}\right)$. Additionally, although the shortest observation time available is $\Delta=6.3 \mathrm{~ms}$, the diffusion spectrum extracted from the multiexponential decay attenuation is only reliable from $\Delta=20 \mathrm{~ms}$ and above due to high experimental uncertainties introduced through weak echo attenuations.

The distribution of diffusion coefficients obtained for $\Delta=20 \mathrm{~ms}$ comprises two peaks (Figure 5.2, numbers 1 and 2) centred at $\sim 2.7 \times 10^{-12} \mathrm{~m}^{2} \mathrm{~s}^{-1}$ and $\sim 1.5 \times 10^{-}$ 
${ }^{11} \mathrm{~m}^{2} \mathrm{~s}^{-1}$, respectively, in addition to the third peak (Figure 5.2, number 3) associated with the unrestricted diffusion of toluene molecules dissolved directly in the continuous phase. To assess whether restricted diffusion contributes to peak 1 and/or peak 2, the mean square displacement $\left\langle\mathrm{Z}^{2}\right\rangle$ of both droplets and oil molecules upon increasing the observation time must be considered (Eq.3.14). Unlike unrestricted diffusion, the mean square displacement is independent of the observation time upon restricted diffusion, i.e. the product $D \Delta$ is constant. As such, when restricted diffusion occurs, the diffusion spectrum is expected to shift to slower diffusion coefficient values upon increasing the observation time, i.e. the apparent droplet size increases. Figure 5.3 shows the plot of the product $D \Delta$ as a function of the observation time, $\Delta$, for peak 1 .

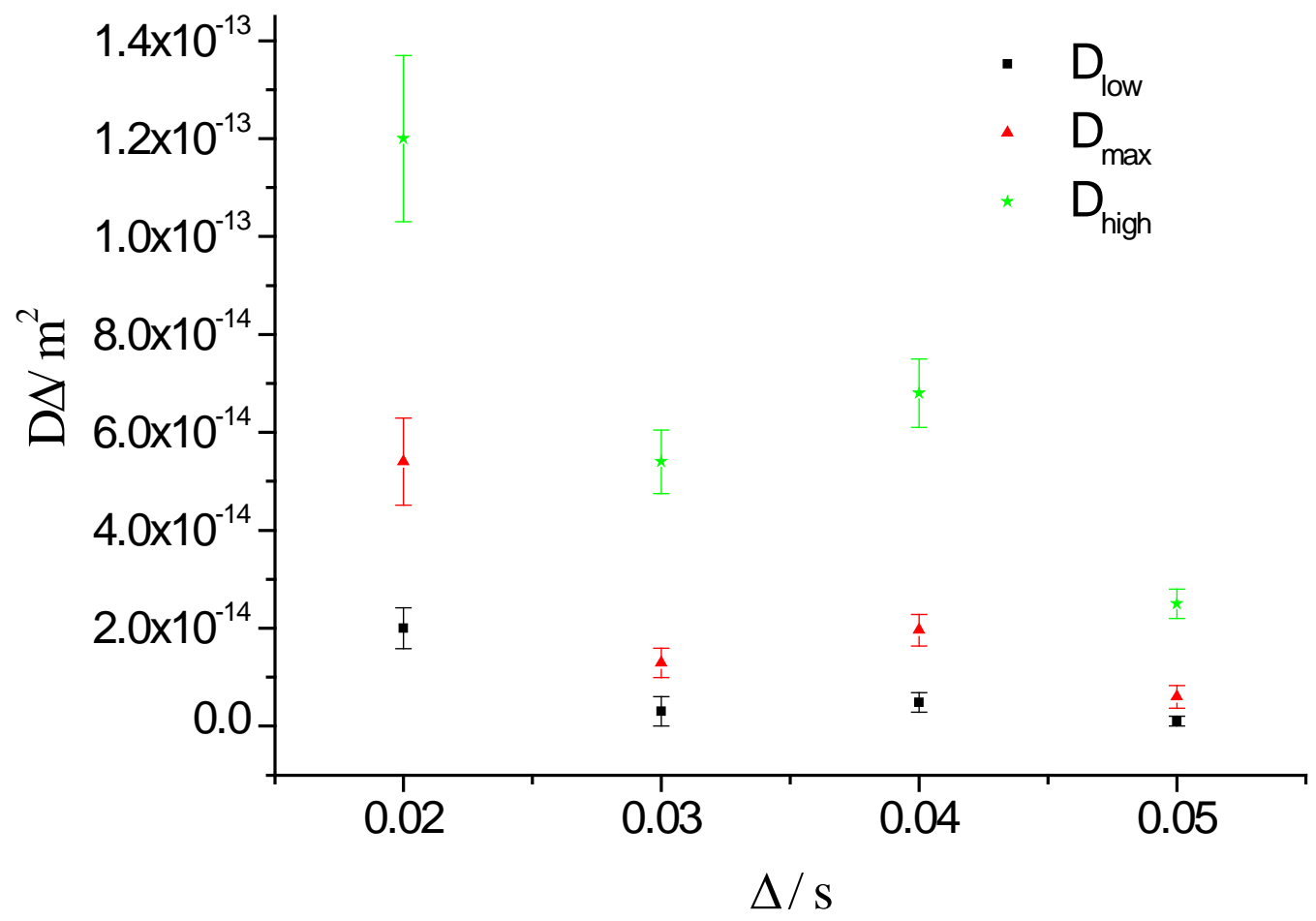

Figure 5.3. Product $D \Delta$ versus $\Delta$ for the low end tail, $D_{\text {low }}$, maximum, $D_{\max }$ and high end tail, $D_{\text {high }}$, of the slower diffusing peak (number 1, Figure 5.2) of the toluene diffusion spectrum obtained for a fresh $\mathbf{4 0} \mathrm{wt} \%$ toluene sample.

For times longer than $20 \mathrm{~ms}$, the mean square displacement $<\mathrm{Z}^{2}>$ associated with the low end tail and the maximum of peak 1 becomes constant (Figure 5.3). The maximum of peak 1 correlates to an average distance travelled of $0.16 \pm 0.03$ $\mu \mathrm{m}$. This distance can be defined as the mean interdroplet spacing, i.e. the 
maximum distance droplets are able to travel, on average, before encountering another droplet and becoming restricted. Note that this value corresponds well with that calculated from the confocal microscopy data. However, droplets with a diameter smaller than the mean interdroplet spacing do not undergo restricted diffusion as these are able to move freely within the aqueous channels, though obstruction effects may be important.

On the other hand, the mean square displacement corresponding to the high end tail is more unstable which may be due to the minor contribution of the unrestricted diffusion of the droplets. Although peak 1 predominantly represents unrestricted diffusion for $\Delta=20 \mathrm{~ms}$, the contribution of restricted diffusion is probably under represented here since two close peaks that lie within one decade of each other cannot be readily resolved in the diffusion spectrum using our algorithm for an inverse Laplace transform [97, 98].

For observation times longer than $20 \mathrm{~ms}$, droplets undergo restricted diffusion due to the presence of other droplets, hence peak 1 shifts to slower diffusion coefficient values and is finally associated only with restricted diffusion of the droplets themselves. Using the SLS droplet size distribution (Figure 4.1) and the Stokes-Einstein equation (Eq. 3.4) for $\Delta=20 \mathrm{~ms}$, peak 1 represents the unrestricted diffusion of droplets of $70 \mathrm{~nm}$ to $0.4 \mu \mathrm{m}$ diameter, i.e. the bulk of the oil droplets in the sample. Droplets in the range $\sim 0.5-3 \mu \mathrm{m}$ diameter represent a very small volume fraction of the dispersed phase, hence they are not readily detected by the spectrometer. The intensity of peak 1 decreases upon increasing $\Delta$ indicating that the contribution of restricted diffusion of the droplets to the total diffusion spectrum decreases. By $60 \mathrm{~ms}$ peak 1 can no longer be detected as it now lies below the detection limit of the instrument $\left(\sim 1 \times 10^{-14} \mathrm{~m}^{2} \mathrm{~s}^{-1}\right)$.

In Figure 5.4 is shown the plot of the product $D \Delta$ versus $\Delta$ for the middle peak (number 2) centred at $\sim 1.5 \times 10^{-11} \mathrm{~m}^{2} \mathrm{~s}^{-1}$ in the diffusion spectrum (Figure 5.2). 


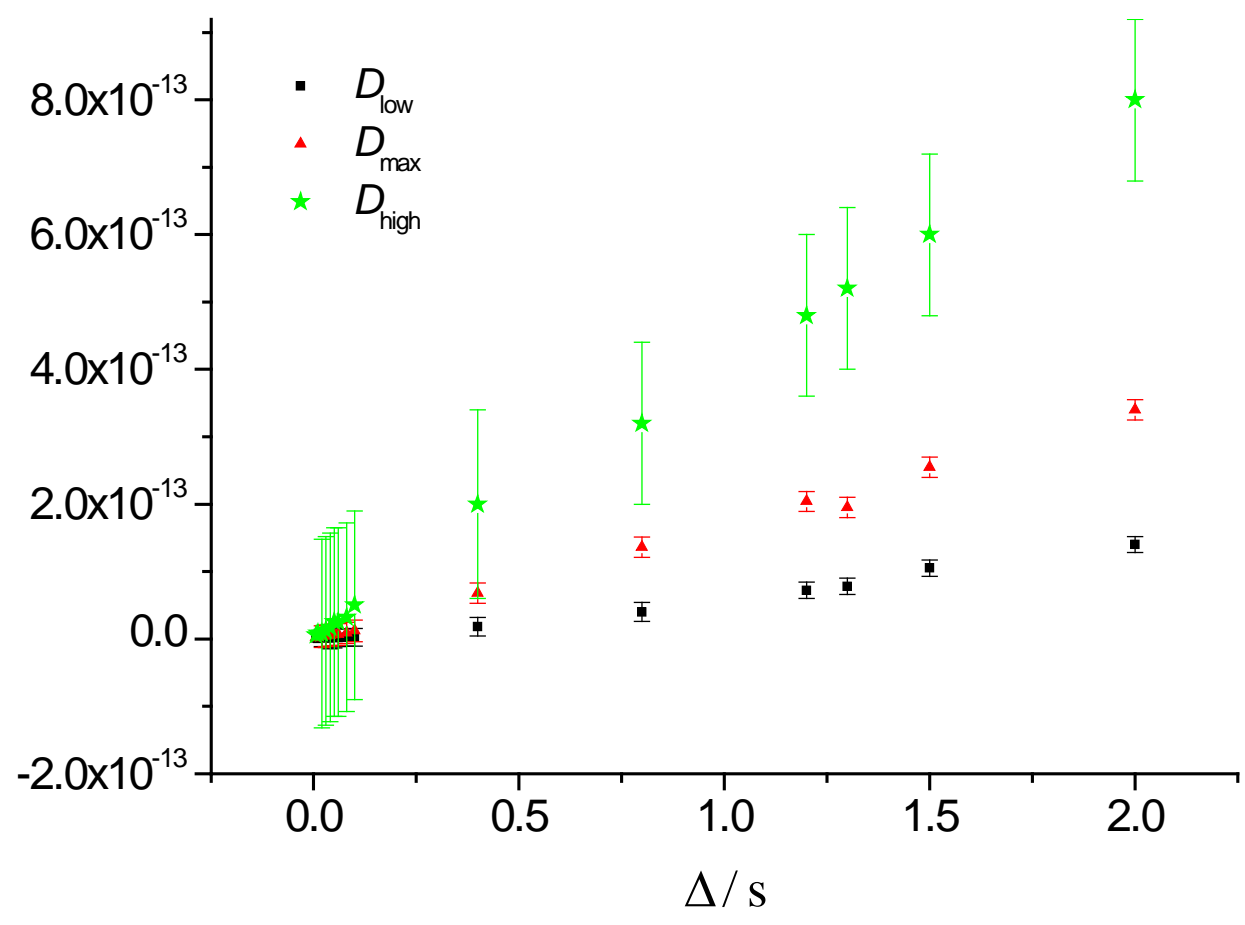

Figure 5.4. Plot of the product $D \Delta$ as a function of $\Delta$ for the low end tail, $D_{\text {low }}$, maximum, $D_{\text {max }}$ and high end tail, $D_{\text {high }}$, of the middle peak (Figure 5.2, number 2 ) of the toluene diffusion spectrum obtained for a fresh $40 \mathrm{wt} \%$ toluene sample.

The product $D \Delta$ is an increasing function of $\Delta$ for all components of peak 2, indicating that this peak is predominantly due to unrestricted diffusion for all values of $\Delta$. Using the SLS droplet size distribution coupled with the StokesEinstein equation for $\Delta=20 \mathrm{~ms}$, peak 2 corresponds to the unrestricted diffusion of oil swollen micelles (10-30 nm, $D=1.5$ to $4 \times 10^{-11} \mathrm{~m}^{2} \mathrm{~s}^{-1}$, high end tail of the peak) as well as the unrestricted diffusion of the small droplets between 35 and 60 $\mathrm{nm}$ diameter (remainder of the peak). Upon increasing $\Delta$ above $20 \mathrm{~ms}$, peak 2 broadens slightly at the lower diffusion coefficient end. This broadening is mainly attributed to the inverse Laplace transform process rather than having a physical origin. With peak 1 shifting to the left upon increasing $\Delta$ due to restricted diffusion of the largest droplets, peak 2 becomes more distinguished and shows the full range of diffusion coefficients associated with pure unrestricted diffusion. Hence, at $\Delta=60 \mathrm{~ms}$, the true low end tail of peak 2 is shown at $\sim 2.4 \times 10^{-12} \mathrm{~m}^{2} \mathrm{~s}^{-}$ ${ }^{1}$ indicating that it represents the unrestricted diffusion of oil swollen micelles and droplets up to $\sim 0.2 \mu \mathrm{m}$. These data indicate that the diffusion of droplets smaller 
than $\sim 0.2 \mu \mathrm{m}$ is unrestricted, which is in agreement with the value of the mean interdroplet spacing determined previously $\left(<\mathrm{Z}_{\text {interdroplet }}>=0.16 \pm 0.03 \mu \mathrm{m}\right)$.

For $\Delta>60 \mathrm{~ms}$, peak 2 progressively narrows around the median value of $D=(1.5$ $\pm 0.3) \times 10^{-11} \mathrm{~m}^{2} \mathrm{~s}^{-1}$ reflecting the slow transition of the echo attenuation from a multiexponential decay to a single exponential decay (Figure 5.1). However, even at $\Delta_{\text {switch }}=1.3 \mathrm{~s}$, when the attenuation decays as a single exponential function of gradient strength, the inverse Laplace transform artificially broadens the peak. That is, the inverse Laplace transform never provides a single delta function. For example, a broad distribution of diffusion coefficients is obtained even for pure water.

\subsection{Effect of oil concentration}

The effect of oil content on the emulsion microstructure and dynamics in the toluene/Triton X-100/water ternary system was monitored by varying the oil concentration from 5 to 70 wt\% while maintaining an oil-to-surfactant weight ratio of 5:1. Oil concentration did not affect the droplet size distribution from 5 to $55 \mathrm{wt} \%$ toluene as evidenced by SLS (Figure 4.1), hence only the droplet concentration increased. As opposed to laser scanning confocal microscopy and cryo-SEM, PFG-NMR experiments evidenced a significant effect on the echo attenuation of the oil signal, the value of the motional averaging of the oil diffusion and the time scale of the oil exchange $\left(\Delta_{\text {switch }}\right)$ upon increasing toluene content.

In Figure 5.5 are highlighted the first few data points of the echo attenuation of the oil signal (fast diffusing portion, see Figure 5.1) when the echo attenuation remains unchanged, i.e. for $\Delta>\Delta_{\text {switch }}$, for emulsions prepared between 5 and 55 wt\% toluene. 


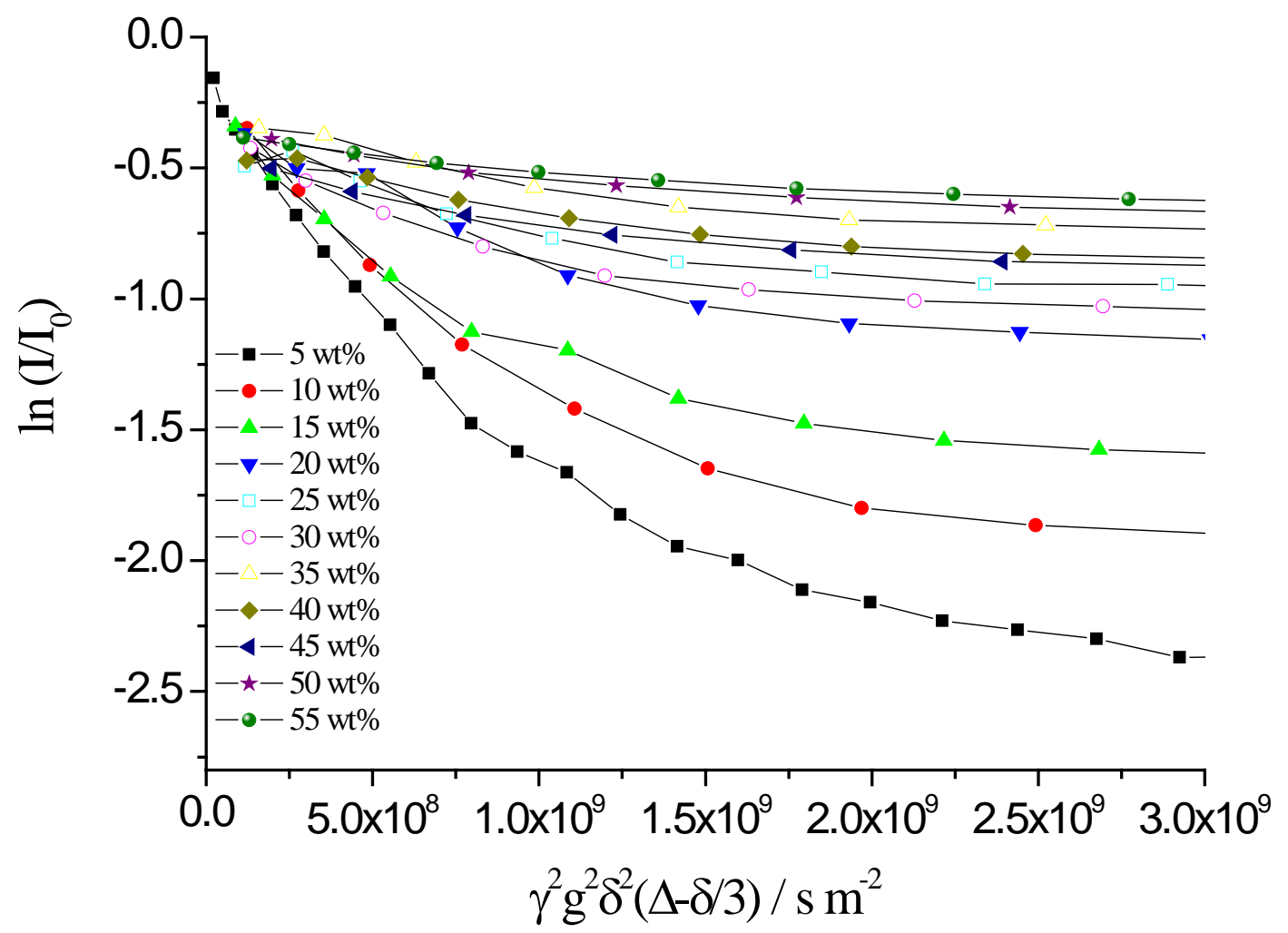

Figure 5.5. Fast diffusing portion of the echo attenuation of the oil signal above $\Delta_{\text {switch }}$ for emulsion samples prepared with toluene between 5 and 55 wt\%. Lines to guide eyes only.

As mentioned in chapter 4, the fast diffusing part of the echo attenuation indicates the presence of toluene molecules diffusing freely in the aqueous continuous phase of the emulsions as toluene is partially soluble in water. For toluene concentrations between 5 and $55 \mathrm{wt} \%$, the contribution of the unrestricted diffusion of toluene molecules in the continuous phase relative to the total attenuation data decreases upon increasing oil content, i.e. upon decreasing the volume fraction of continuous phase (Figure 5.5). The decrease is linear between 5 and $25 \mathrm{wt} \%$ oil but becomes non linear between 30 and $55 \mathrm{wt} \%$ due to the reduction of the number of data points leading to a higher uncertainty associated with the quantitative data extracted from the inverse Laplace transform. At very high toluene concentration, from $\sim 65$ to $70 \mathrm{wt} \%$, this fast diffusing contribution totally disappears.

The diffusion coefficients associated with the unrestricted motion of the toluene molecules dissolved in the continuous phase were estimated using an inverse Laplace transform and these are given in Table 5.1. 


\begin{tabular}{|l|l|}
\hline Oil concentration (wt\%) & $(D \pm 0.1) \times 10^{-9} \mathrm{~m}^{2} \mathrm{~s}^{-1}$ \\
\hline 5 & 2.3 \\
\hline 10 & 2.3 \\
\hline 15 & 2.3 \\
\hline 20 & 2.3 \\
\hline 25 & 2.3 \\
\hline 30 & 1.6 \\
\hline 35 & 1.8 \\
\hline 40 & 1.6 \\
\hline 45 & 1.6 \\
\hline 50 & 1.8 \\
\hline 55 & 1.3 \\
\hline 60 & 1.0 \\
\hline 65 & None \\
\hline 70 & None \\
\hline
\end{tabular}

Table 5.1. Diffusion coefficient of toluene molecules dissolved in the aqueous continuous phase as a function of oil concentration between 5 and $70 \mathrm{wt} \%$ obtained using an inverse Laplace transform.

The diffusion coefficient of pure toluene dissolved directly in the continuous phase is a decreasing function of oil concentration spanning from $\sim 1$ to $\sim 2.3 \times 10^{-}$ ${ }^{9} \mathrm{~m}^{2} \mathrm{~s}^{-1}$. This is mainly caused by obstruction effects of the surrounding droplets, i.e. toluene molecules dissolved in the continuous phase are forced to move around the droplets, which slows them down. As the number of droplets increases with oil concentration so does the system tortuosity leading to a decreasing apparent diffusion coefficient of the toluene molecules (Eq. 3.14). Obstruction effects of oil droplets similarly lead to a decrease of the apparent diffusion coefficient associated with the unrestricted diffusion of the water molecules [40]. Additionally, the echo attenuation of the oil signal above $\Delta_{\text {switch, }}$ i.e. when motional averaging of the oil diffusion is measured, is also affected by toluene concentration (Figure 5.6 and Figure 5.7). 


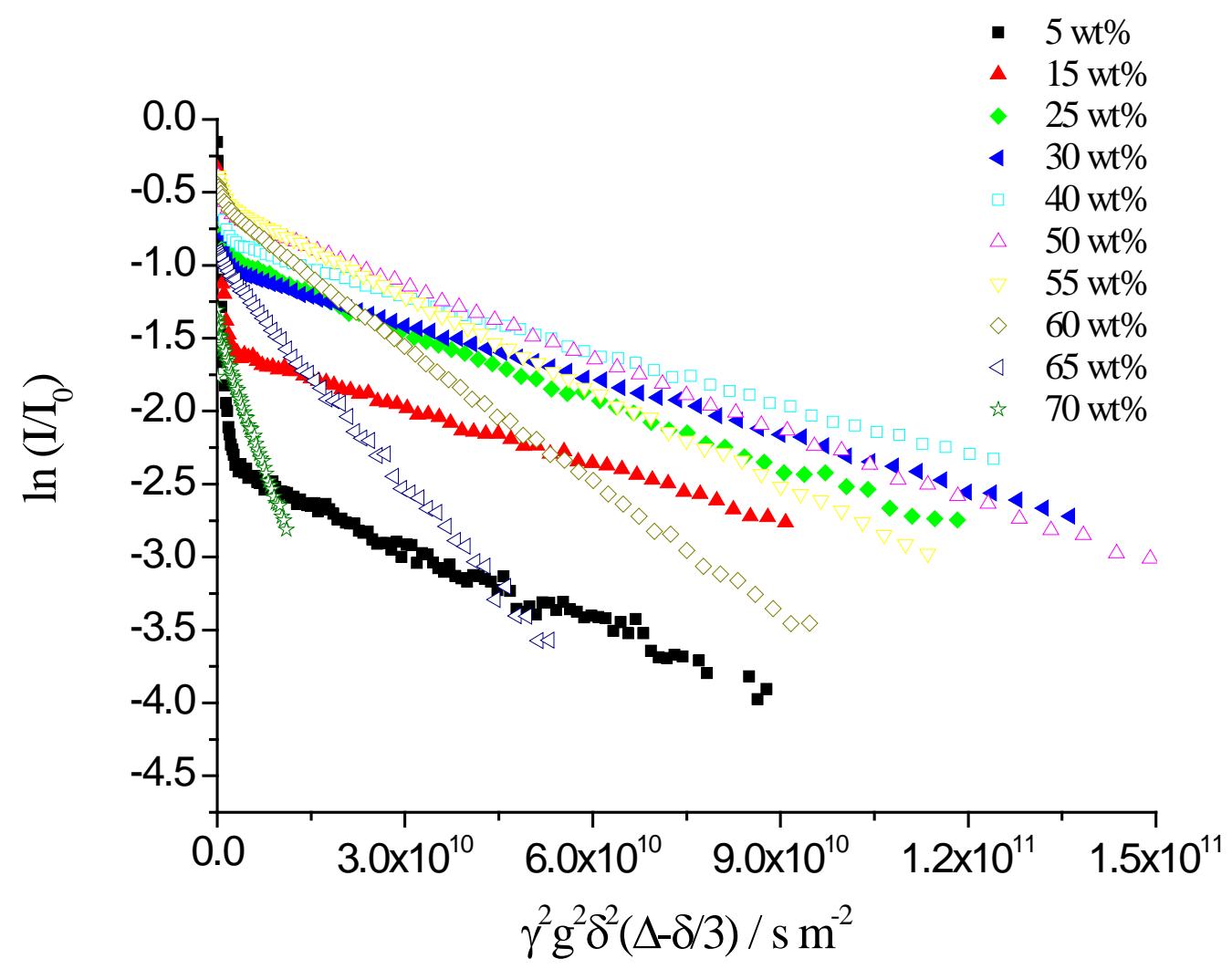

Figure 5.6. Diffusion echo attenuation of the oil signal above $\Delta_{\text {switch }}$ (single exponential decay) for emulsion samples prepared with toluene between 5 and $70 \mathrm{wt} \%$.

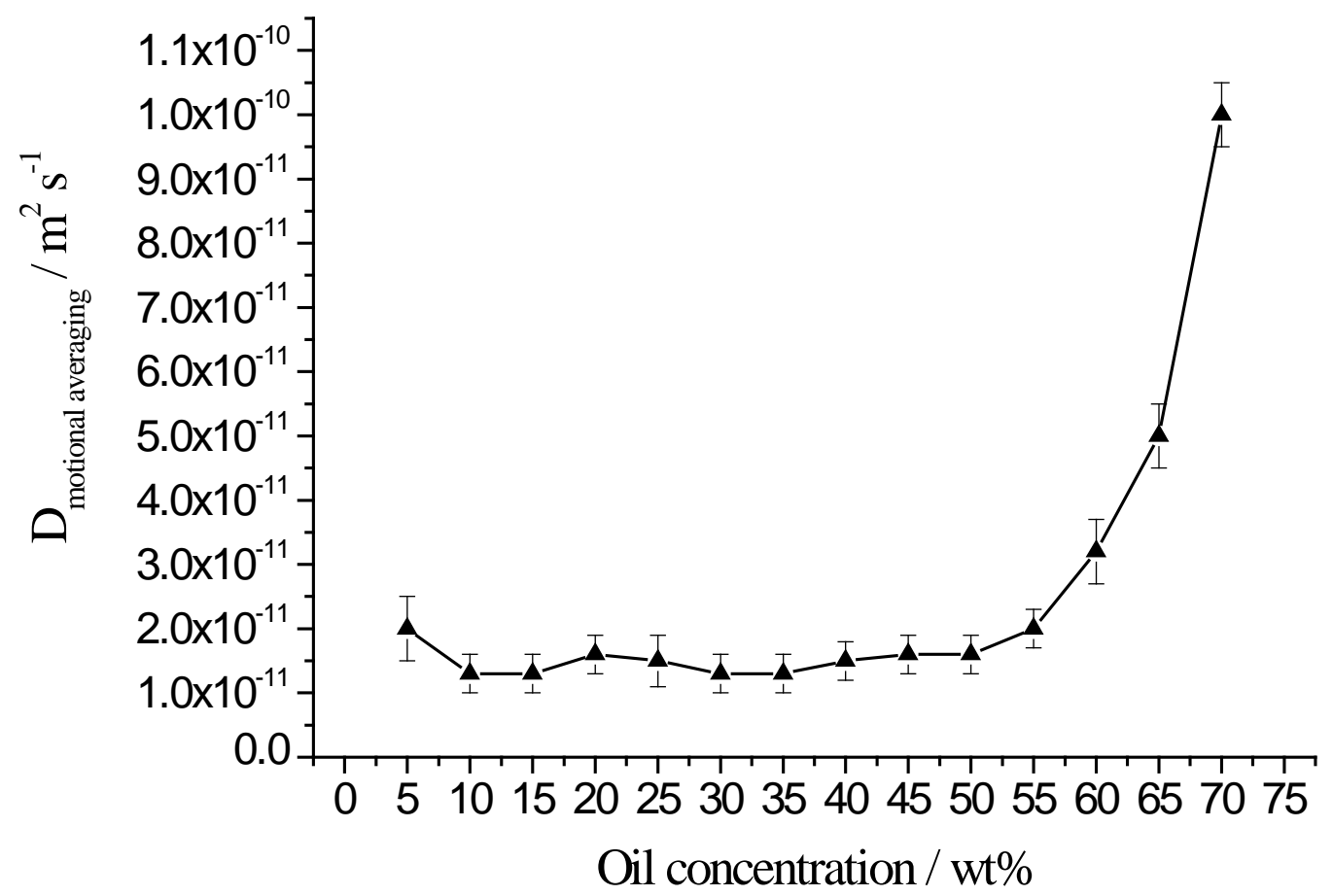

Figure 5.7. Motional averaging of the oil diffusion versus oil concentration between 5 and 70 wt \% for fresh toluene/Triton $\mathrm{X}-100$ /water emulsions. 
From 5 to $70 \mathrm{wt} \%$ toluene, the motional averaging estimated from the inverse Laplace transforms varies between $(1.3 \pm 0.3) \times 10^{-11}$ and $(1.0 \pm 0.5) \times 10^{-10} \mathrm{~m}^{2}$ $\mathrm{s}^{-1}$ (Figure 5.7).

From 5 to $\sim 55$ wt\% toluene, the value of the motional averaging is, within uncertainty, independent of oil concentration with an average value of $D=(1.5 \pm$ 0.1) $\times 10^{-11} \mathrm{~m}^{2} \mathrm{~s}^{-1}$ despite the droplets having the same size over the entire concentration range (Figure 4.1). Since the motional averaging is proportional to the mean square displacement of the oil phase over the duration $\Delta$, upon increasing the droplet concentration and the frequency of collisions, individual oil molecules are expected to exchange, on average, a larger number of times over the same duration, leading to the motional averaging of the oil diffusion being an increasing function of toluene concentration. Since this is not observed, oil exchange must be reduced, i.e. exchange becomes more difficult upon increasing oil concentration. The increase of the droplet encounter frequency is balanced by a more rigid interfacial domain at higher oil content resulting in the motional averaging remaining approximately constant upon increasing oil concentration.

Between $\sim 60$ and $70 \mathrm{wt} \%$ oil, the steep increase of the motional averaging value from $(3.2 \pm 0.5) \times 10^{-11}$ to $(1.0 \pm 0.5) \times 10^{-10} \mathrm{~m}^{2} \mathrm{~s}^{-1}$ is related to the $60 \%$ increase of the mean droplet size as compared with the more dilute samples (Figure 4.1) thereby increasing the contribution of oil diffusion within a single droplet before exchanging. That is, larger oil droplets correlate to a longer distance travelled by oil molecules within these droplets during oil exchange leading to a faster motional averaging value.

The evolution of the diffusion spectrum of the dispersed oil phase is now considered upon increasing the observation time, $\Delta$, for times shorter than $\Delta_{\text {switch, }}$ i.e. before oil exchange is detected. Here, the polydispersity of the droplets and oil swollen micelles is probed (Figure 5.8 and Figure 5.10) 


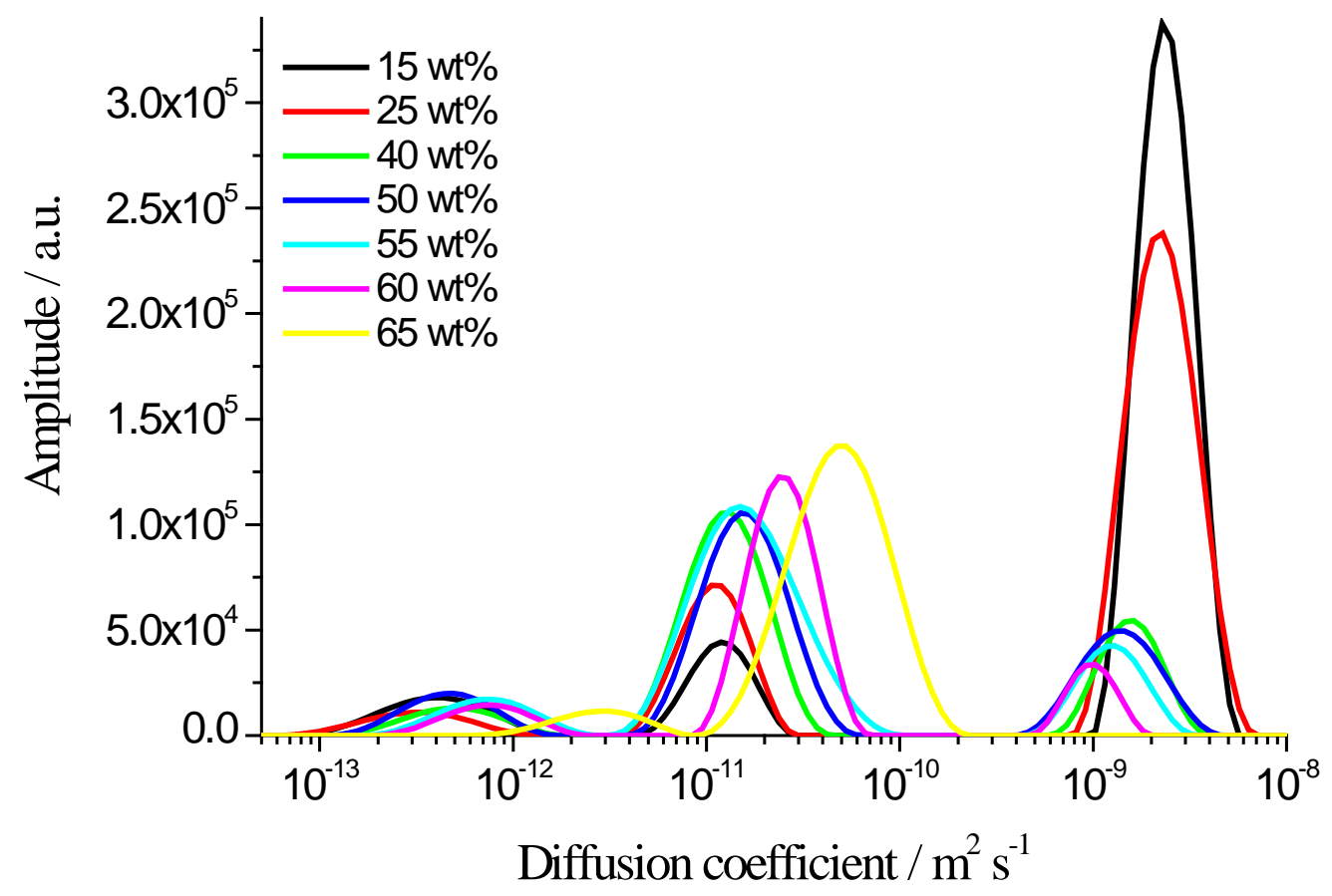

Figure 5.8. Diffusion spectra of the oil signal for emulsions prepared with toluene between 15 and $65 \mathrm{wt} \%$ toluene, $\Delta=40 \mathrm{~ms}$.

For $\Delta=40 \mathrm{~ms}$, upon increasing oil concentration from 15 to $65 \mathrm{wt} \%$, the amplitude of the peak corresponding to the fastest diffusion, centered between $\sim 1.0 \times 10^{-9}$ and $\sim 2.3 \times 10^{-9} \mathrm{~m}^{2} \mathrm{~s}^{-1}$ and attributed to the unrestricted diffusion of toluene molecules in the continuous phase, is reduced until it totally disappears from $65 \mathrm{wt} \%$ oil as discussed earlier (see Figure 5.5 and Table 5.1).

The position of the peak corresponding to the slowest diffusion, centered at $\sim 4.0$ $\times 10^{-13} \mathrm{~m}^{2} \mathrm{~s}^{-1}$ and associated with the restricted diffusion of the droplets between $\sim 0.3$ and $\sim 0.5 \mu \mathrm{m}$ diameter (see section 5.2), remains unchanged from 15 to $\sim 60$ wt\% toluene. Upon increasing concentration, the spacing between the droplets decreases and restriction becomes enhanced. This should result in the peak corresponding to the slowest diffusion being shifted to the left from 15 to 60 wt\% toluene. Because the peak corresponding to the slowest diffusion is close to the spectrometer detection limit $\left(\sim 1 \times 10^{-14} \mathrm{~m}^{2} \mathrm{~s}^{-1}\right)$ and is of very low intensity relative to the other peaks, what is observed is that between $\sim 55$ and $\sim 60 \mathrm{wt} \%$ oil, the peak corresponding to the slowest diffusion is slightly shifted to higher diffusion coefficients due to the decrease of the contribution of the restricted diffusion of the droplets (droplets larger than $\sim 0.5 \mu \mathrm{m}$ are no longer detected) and the concomitant increase of the contribution of the restricted diffusion of oil 
molecules within droplets between $\sim 0.3$ and $\sim 1 \mu \mathrm{m}$ in diameter (Eq. 3.15). The detection of the restricted diffusion of the oil molecules within the droplets is in agreement with the SLS data since the volume fraction of droplets between $\sim 0.5$ and $3 \mu \mathrm{m}$ diameter increases from $\sim 60 \mathrm{wt} \%$ toluene (Figure 4.1).

Moving on to emulsions prepared with toluene between $\sim 65$ and $70 \mathrm{wt} \%$, there is no peak in the diffusion range of $\sim 10^{-13} \mathrm{~m}^{2} \mathrm{~s}^{-1}$ meaning that the diffusion associated with the restricted diffusion of droplets larger than $\sim 0.3 \mu \mathrm{m}$ is too slow to be detected at $\Delta=40 \mathrm{~ms}$ in these highly packed samples.

The product $D \Delta$ for all the components of the peak associated with slowest diffusion, centered now at $\sim 3.0 \times 10^{-12} \mathrm{~m}^{2} \mathrm{~s}^{-1}$, is an increasing function of $\Delta$ (Figure 5.9) indicating that the smallest droplets still undergo unrestricted diffusion since their diameter is smaller than the mean interdroplet spacing. Using the Stokes-Einstein equation (Eq. 3.4), the unrestricted diffusion contribution to this peak pertains to droplets between $\sim 40 \mathrm{~nm}$ and $\sim 0.4 \mu \mathrm{m}$ diameter for a $65 \mathrm{wt} \%$ toluene emulsion.

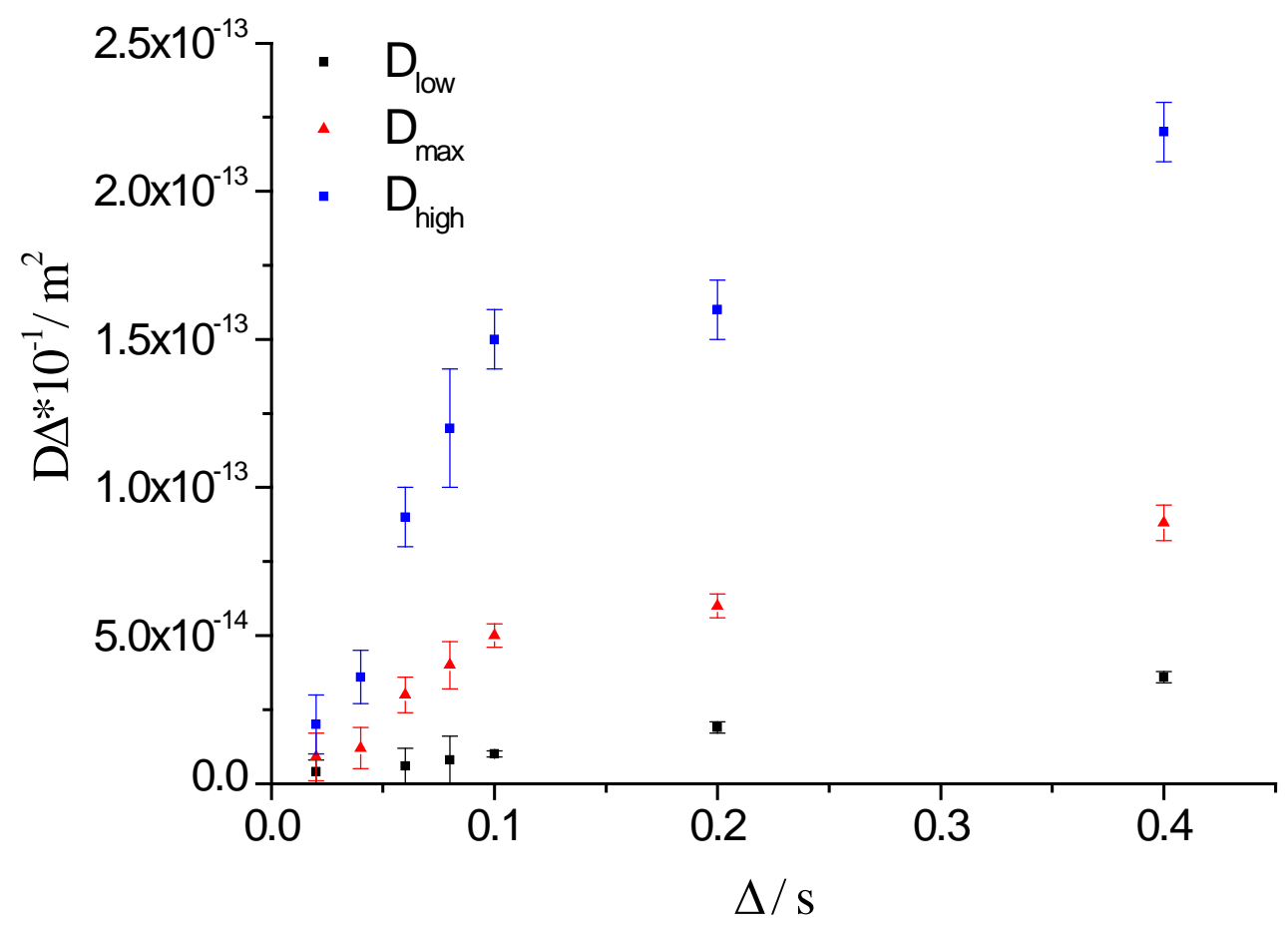

Figure 5.9. Plot of the product $D \Delta$ as a function of $\Delta$ for the low end tail, $D_{\text {low }}$, maximum, $D_{\text {max }}$ and high end tail, $D_{\text {high }}$, of the peak associated with slowest diffusion in the toluene diffusion spectrum obtained for a fresh $65 \mathrm{wt} \%$ toluene sample. 
Despite the product $D \Delta$ being an increasing function of the observation time, $\Delta$, the peak corresponding to the slowest diffusion does shift to slower diffusion coefficient values upon increasing $\Delta$ from 60 to $400 \mathrm{~ms}$ as illustrated by the decrease of the slope in the plot $D \Delta$ vs $\Delta$ (Figure 5.9). This means that restricted diffusion is also measured, corresponding to oil molecules diffusing within droplets of $\sim 0.7$ to $\sim 3 \mu \mathrm{m}$ diameter (Eq. 3.15) but is a minor contribution to the overall peak intensity.

Moving on to the analysis of the middle peak for $\Delta=40 \mathrm{~ms}$ (Figure 5.8), between 15 and $\sim 55 \mathrm{wt} \%$ toluene, it is centered at $\sim 1.5 \times 10^{-11} \mathrm{~m}^{2} \mathrm{~s}^{-1}$ and represents the unrestricted diffusion of oil swollen micelles and droplets up to $\sim 0.2 \mu \mathrm{m}$. Note that the high end tail of this middle peak shifts to faster diffusion coefficients upon increasing oil content because the peak corresponding to the fastest diffusion, attributed to the unrestricted diffusion of toluene molecules in the continuous phase, moves simultaneously to slower diffusion coefficients as a result of the obstruction effects imposed by the presence of the droplets. When two peaks are separated by less than an order of magnitude, a part of one peak may be transferred to the other peak during the inverse Laplace transform, hence boundaries and intensities are often misweighted.

Upon increasing oil concentration to $60 \mathrm{wt} \%$, the inverse Laplace artifact is so significant that the whole middle peak moves to faster diffusion coefficient values with a concurrent increase of amplitude. From $65 \mathrm{wt} \%$ oil, the peak associated with fastest diffusion has totally been incorporated into the middle peak to form one single peak. On a first approach, one could think of the detection of the restricted diffusion of oil molecules within very large droplets instead of an inverse Laplace artifact to account for the shift of the middle peak to the right but this would correspond to diffusion within droplets as large as $12 \mu \mathrm{m}$ diameter for a $65 \mathrm{wt} \%$ toluene sample (Eq. 3.15). This does not account for the SLS data which do not indicate the presence of such large droplets until $\sim 6 \mathrm{~h}$ post emulsification. Moreover, the product $D \Delta$ for the middle peak between $\sim 60$ and $70 \mathrm{wt} \%$ is an increasing function of $\Delta$, which points out that unrestricted diffusion is still the main contributor of the peak. As such, between $\sim 60$ and $70 \mathrm{wt} \%$ oil, the middle peak mainly represents the unrestricted diffusion in the continuous phase 
of both oil swollen micelles (10-30 nm diameter) and a portion of the toluene molecules.

Upon increasing $\Delta$ to $60 \mathrm{~ms}$, the diffusion coefficients corresponding to the restricted diffusion of the droplets themselves are too slow to be detected except for the lowest toluene concentration of $15 \mathrm{wt} \%$ (Figure 5.10)

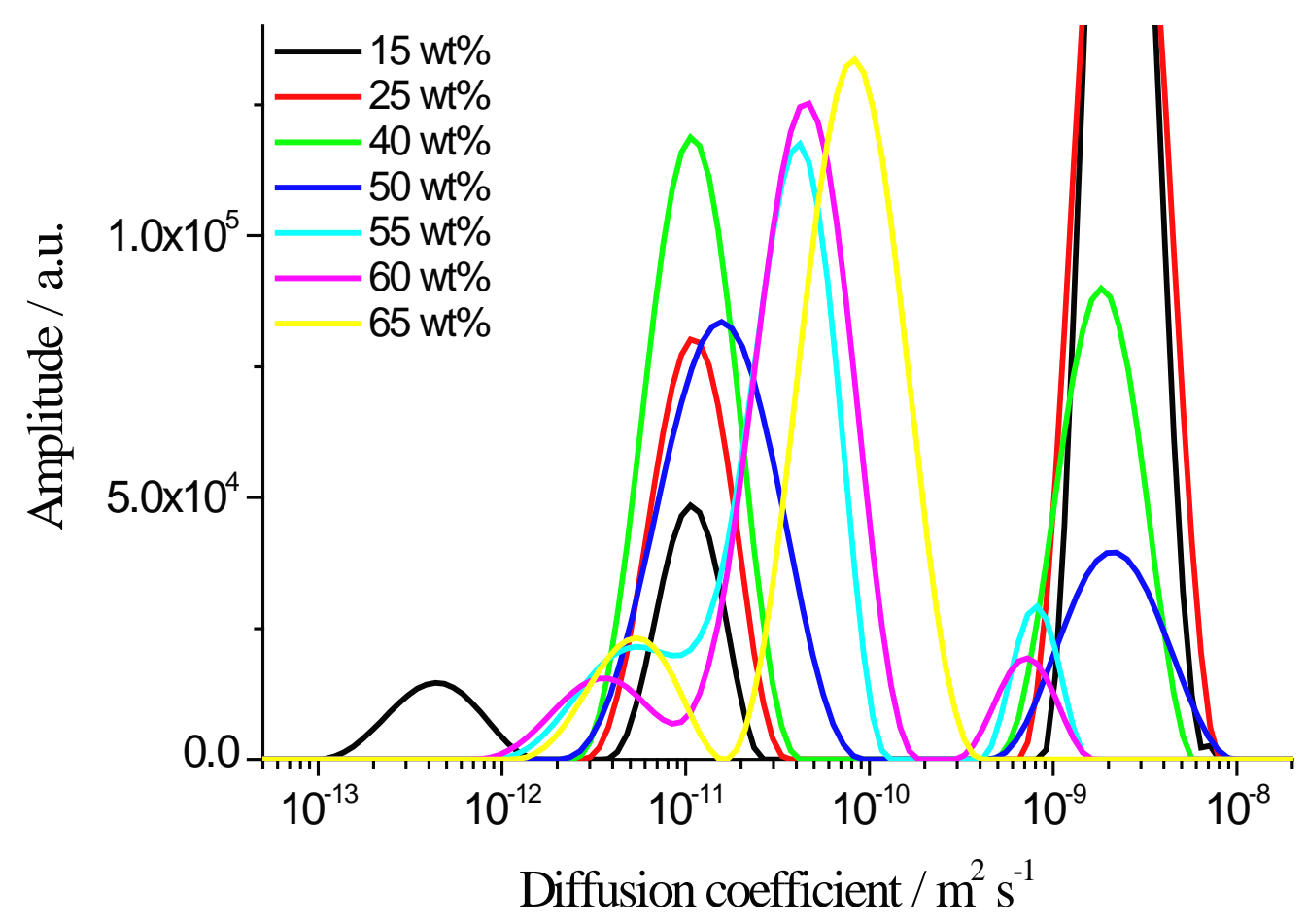

Figure 5.10. Diffusion spectra of the oil signal for emulsions prepared with toluene between 15 and $65 \mathrm{wt} \%, \Delta=60 \mathrm{~ms}$. The fast diffusing peaks for both the 15 and $25 \mathrm{wt} \%$ oil samples have been cut for better visualization of the remaining peaks.

From $\sim 25$ to $\sim 50$ wt\% toluene, the motion of the dispersed oil phase is represented by a single peak centered at $\sim 1.0 \times 10^{-11} \mathrm{~m}^{2} \mathrm{~s}^{-1}$ for $\Delta=60 \mathrm{~ms}$ (Figure 5.10) including the unrestricted diffusion of the oil swollen micelles and droplets up to $\sim 0.15 \mu \mathrm{m}$ (see section 5.2).

From 55 to $65 \mathrm{wt} \%$ toluene, the further shift of the peak corresponding to the fastest diffusion to slower diffusion coefficients led to the separation of the middle peak into two distinct peaks. As such, the contribution of the unrestricted diffusion of the smallest droplets $(\sim 40 \mathrm{~nm}-0.4 \mu \mathrm{m})$ coupled to the restricted diffusion of oil molecules within the largest droplets ( $\sim 0.7$ to $3 \mu \mathrm{m}$ ) become fully 
distinguished from that of the unrestricted diffusion of both the oil swollen micelles and a portion of the toluene molecules in the continuous phase.

For toluene between 5 and $\sim 50 \mathrm{wt} \%$, the single diffusion peak progressively narrows upon further increasing $\Delta$ until motional averaging of the oil diffusion is measured. The process is slower between $\sim 55$ and $70 \mathrm{wt} \%$ oil, i.e. the peak corresponding to the slowest diffusion continuously disappears before the middle peak progressively narrows. Thus, based on a qualitative analysis of the diffusion spectrum upon increasing $\Delta$ at very high oil content (55- $70 \mathrm{wt} \%$ ), the transition from a multiexponential decay, when the individual droplet and molecule motions are probed, to the motional averaging of the diffusion, occurs on a longer time scale compared with the more diluted emulsions (5 - $55 \mathrm{wt} \%)$.

Taking a more quantitative approach, the plot of the switching time $\Delta_{\text {switch }}$ versus oil concentration (Figure 5.11) stresses that the timescale of the oil exchange between emulsion droplets is affected by the toluene concentration between 5 and $70 \mathrm{wt} \%$.

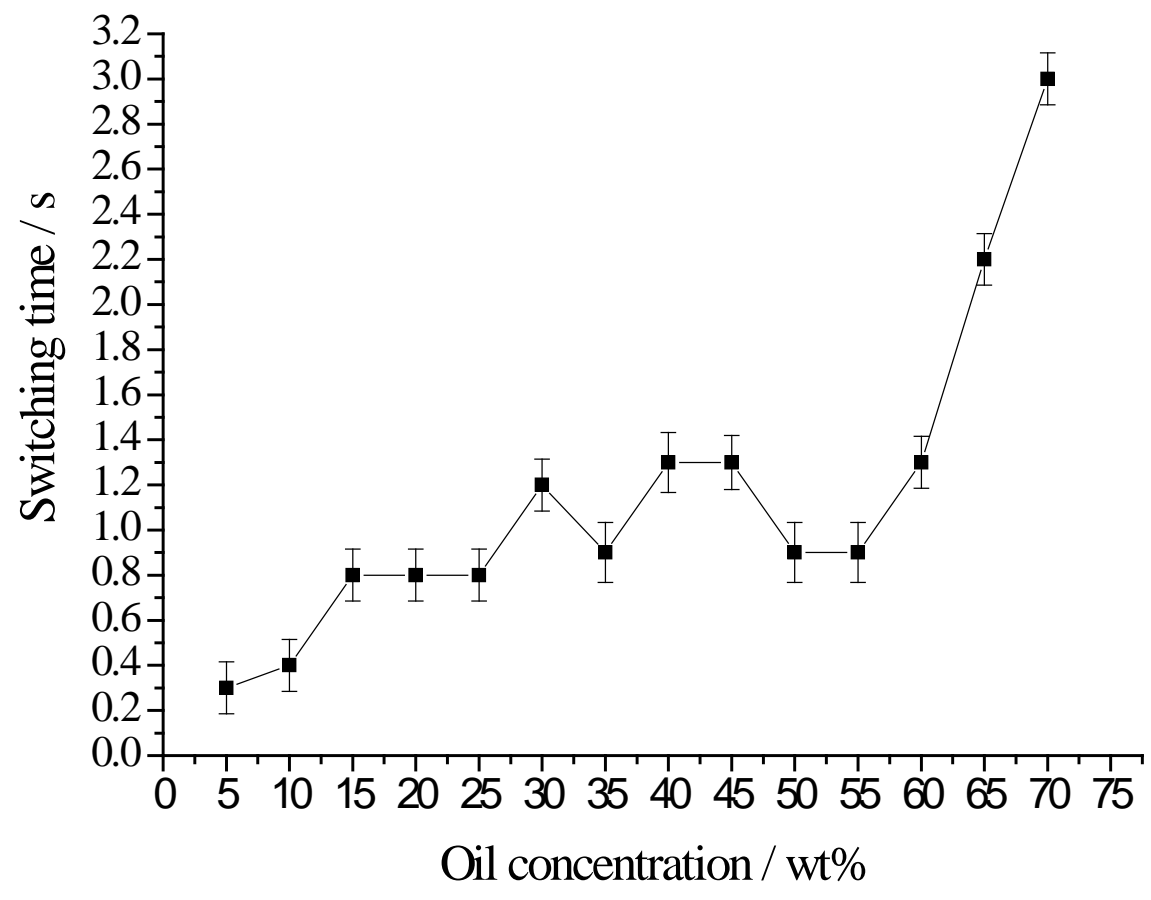

Figure 5.11. NMR switching time $\Delta_{\text {switch }}$ versus oil concentration for fresh toluene/Triton $\mathrm{X}$ 100/water emulsions prepared between 5 and $70 \mathrm{wt} \%$ toluene.

Firstly, the time scale of the oil exchange, i.e. $\Delta_{\text {switch, }}$ is an increasing function of oil concentration, varying from $\sim 300 \mathrm{~ms}$ to $\sim 3$ s. Upon increasing oil 
concentration from 5 to $\sim 55 \mathrm{wt} \%$ oil, without concomitantly changing the droplet size (see SLS data, Figure 4.1), the droplets must be closer to each other due to the increased number of droplets present in the sample. This should lead to the switching time $\Delta_{\text {switch }}$ being a decreasing function of concentration as opposed to the increasing function observed, since droplets will encounter each other on average more frequently in more highly concentrated samples, hence all other things being equal, oil transfer should be facilitated. The fact that the switching time $\Delta_{\text {switch }}$ is an increasing function of concentration although the size distribution is identical and the droplets are closer to each other indicates that the characteristics of the emulsifier thin film, i.e. droplet-droplet and wateremulsifier-oil interactions must be modified in this system upon varying oil concentration. Since $\Delta_{\text {switch }}$ measures the ease with which the oil exchange process occurs, it must depend on the properties of the interfacial domain.

Despite maintaining a 1:5 surfactant-to-oil weight ratio and the same droplet size distribution, three concentration domains have been previously identified within the toluene concentration range of 5 to $55 \mathrm{wt} \%$ [41]. These three domains are a low-oil content dispersed droplet O/W emulsion between 5 and 25 wt\% toluene, a transition domain between $\sim 25$ and $~ 35$ wt $\%$ toluene where a bicontinuous microstructure can be stabilised at low energy input, using a vortex mixer, for 30 wt\% oil [40] and a high-oil content dispersed droplet O/W emulsion between 35 and $\sim 55$ wt\% toluene. The low-oil content and high-oil content regions show vastly different characteristics in terms of macroscopic phase separation behaviour (detailed in chapter 6) and response to energy input [41] suggesting that the properties of the interfacial domain, such as the degree of surfactant packing at the oil/water interface, must be different in the two regions.

At very high toluene content, between $\sim 60$ and $70 \mathrm{wt} \%$ toluene, a fourth concentration domain identified as a closed-cell biliquid foam superposed on a dispersed droplet $\mathrm{O} / \mathrm{W}$ has been previously investigated by Liu and coworkers [40]. From Figure 5.11, a sharp increase of the switching time is observed from $\sim 1.3$ (60 wt\%) to $\sim 3 \mathrm{~s}(70 \mathrm{wt} \%)$. Several processes might contribute to this longer time scale of the oil exchange compared to the more dilute emulsions since $\Delta_{\text {switch }}$ depends on both the rate of droplet diffusion and the properties of the interfacial domain. The glassy structure of the closed-cell foam oil domains [40] 
considerably hinders the oil exchange process by providing a rigid interface and the larger mean size of the oil droplets (Figure 4.1) leads to a slower average droplet motion, hence the reduction of the rate of oil exchange. Finally, such a high droplet packing must decrease the frequency of collisions, one droplet being able to collide only with its close neighbours.

\subsection{Addition of background electrolyte}

Emulsion droplets carry a net charge even in the presence of a nonionic surfactant such as Triton $\mathrm{X}-100$ due to the adsorption of ions from the aqueous continuous phase (mainly $\mathrm{OH}^{-}$and $\mathrm{H}_{3} \mathrm{O}^{+}$ions for MilliQ water). Cations are more strongly hydrated in bulk solution and so compete less effectively for surface sites than the more weakly hydrated anions so preferential adsorption of anions usually leads to a net negative charge on emulsion droplets. The negative charge at the surface of oil droplets in toluene/Triton X-100/water emulsions was confirmed by measuring the zeta potential of the droplets, i.e. the surface charge, upon varying oil concentration using a Zetasizer nano-ZS from Malvern Instruments (section 3.4.2). Emulsions were placed in a folded capillary cell after being diluted 10, 40, 100 and 200 times for samples containing 5, 15, 40 and 55 wt\% toluene, respectively. The voltage applied was $148 \mathrm{~V}$. The refractive index and absorption coefficient of the dispersed phase were taken as 1.390 and 0.001 , respectively. The dispersant viscosity ( $0.8872 \mathrm{cP}$ for water) was taken as the sample viscosity. The zeta potential was calculated using the Smoluchowski model $\left(f\left(K_{a}\right)=1.5\right)$ (section 3.4.7.2).

The zeta potential values in Table 5.2 confirm that toluene/Triton X-100/water emulsion droplets carry a small negative charge which is independent of oil concentration between 5 and $55 \mathrm{wt} \%$ toluene. The mean zeta potential is $-9.55 \pm$ $4 \mathrm{mV}$ indicating that the stability of the droplets via electrostatic repulsion is, as expected, weak since $|\xi|<30 \mathrm{mV}$ is considered as a too weak charge to have a significant effect on emulsion stability via electrostatic repulsion. 


\begin{tabular}{|l|l|l|l|l|}
\hline Oil concentration wt\% & 5 & 15 & 40 & 55 \\
\hline$\xi$ potential / mV & -8.75 & -11.7 & -8.10 & -9.66 \\
\hline
\end{tabular}

Table 5.2. Measurement of the zeta potential of emulsions prepared with 5, 15, 40 and 55 wt \% toluene.

In order to assess the effect of salt on the dynamics of toluene/Triton X-100/water emulsions, $\mathrm{NaCl}$ was solubilised in the aqueous phase of a $15 \mathrm{wt} \%$ toluene emulsion at various concentrations prior to emulsification. For four different electrolyte concentrations between $8.3 \times 10^{-5}$ and $4.1 \times 10^{-3} \mathrm{~mol} \mathrm{~L}^{-1}$, the zeta potential of the droplets was measured (Table 5.3).

\begin{tabular}{|l|l|l|l|l|l|}
\hline $\begin{array}{l}\text { Celectrolyte } \\
\mathrm{mol} \mathrm{L}^{-1}\end{array}$ & 0 & $8.3 \times 10^{-5}$ & $2.5 \times 10^{-4}$ & $4.1 \times 10^{-4}$ & $4.1 \times 10^{-3}$ \\
\hline$\xi_{\mathrm{NaCl}} / \mathrm{mV}$ & $-11.7 \pm 0.8$ & $-10.6 \pm 0.7$ & $-9.5 \pm 0.6$ & $-8.6 \pm 0.9$ & $-5.9 \pm 0.8$ \\
\hline
\end{tabular}

Table 5.3. Zeta potential of a $15 \mathrm{wt} \%$ toluene emulsion upon addition of $\mathrm{NaCl}$ at various concentrations between $8.3 \times 10^{-5}$ and $4.1 \times 10^{-3} \mathrm{~mol} \mathrm{~L}^{-1}$.

From Table 5.3, it appears that the absolute value of the zeta potential for a 15 $w t \%$ toluene emulsion is a decreasing function of electrolyte concentration between $8.3 \times 10^{-5}$ and $4.1 \times 10^{-3} \mathrm{~mol} \mathrm{~L}^{-1}$. This result is consistent with the electrical double layer model from Stern (section 2.3.2) which suggests that in the presence of electrolyte in the vicinity of negatively charged oil droplets $(\xi=$ $-11.7 \pm 0.8 \mathrm{mV}$ without electrolyte, Table 5.3), cations $\left(\mathrm{Na}^{+}\right.$in our experiments) act as counterions within the Stern and diffuse layers to maintain electroneutrality (Figure 2.7). As such, the charge at the droplet surface must decrease in absolute value upon increasing electrolyte concentration. The zeta potential absolute value decreases from 10.6 to $5.9 \mathrm{mV}$ upon increasing electrolyte concentration between $8.3 \times 10^{-5}$ and $4.1 \times 10^{-3} \mathrm{~mol} \mathrm{~L}^{-1}$, i.e. the zeta potential absolute values remain smaller than $\sim 30 \mathrm{mV}$, which indicates that electrostatic repulsive forces are still negligible with respect to the stability of these emulsions.

In PFG-NMR experiments, an effect of $\mathrm{NaCl}$ addition on the oil exchange process was detected. A switch between a single exponential and a multiexponential decay of the echo attenuation as a function of the gradient strength was still 
measured in the presence of $\mathrm{NaCl}$ but the time scale of oil exchange $\left(\Delta_{\text {switch }}\right)$ was altered upon varying electrolyte concentration (Table 5.4).

\begin{tabular}{|l|l|l|l|l|l|}
\hline $\begin{array}{l}\text { Celectrolyte / } \\
\mathrm{mol} \mathrm{L}^{-1}\end{array}$ & 0 & $8.3 \times 10^{-5}$ & $2.5 \times 10^{-4}$ & $4.1 \times 10^{-4}$ & $4.1 \times 10^{-3}$ \\
\hline$\Delta_{\text {switch NaCl }} / \mathrm{s}$ & 0.8 & 0.8 & $>3$ & 1.8 & 1 \\
\hline
\end{tabular}

Table 5.4. NMR switching time between a single and a multiexponential decay of the echo attenuation of the oil signal for a $15 \mathrm{wt} \%$ toluene emulsion upon addition of $\mathrm{NaCl}$ between $8.3 \times 10^{-5}$ and $4.1 \times 10^{-3} \mathrm{~mol} \mathrm{~L}^{-1}$.

In the absence of background electrolyte, the NMR switching time $\Delta_{\text {switch }}$ for a toluene/Triton X-100/water emulsion prepared with $15 \mathrm{wt} \%$ toluene is $~ 800 \mathrm{~ms}$ (Table 5.4). In the presence of $\mathrm{NaCl}$ at a concentration of $\sim 8.3 \times 10^{-5} \mathrm{~mol} \mathrm{~L}^{-1}$, no change in the switching time was observed whereas upon increasing the salt concentration to $\sim 2.5 \times 10^{-4} \mathrm{~mol} \mathrm{~L}^{-1}$, no motional averaging of the oil diffusion was detected for values of the observation time, $\Delta$, up to $\sim 3 \mathrm{~s}$ (upper limit of the pulse sequence). Above this concentration, the switching time decreased passing through $\sim 1.8 \mathrm{~s}$ for a sodium chloride concentration of $\sim 4.1 \times 10^{-4} \mathrm{~mol} \mathrm{~L}^{-1}$ down to $\sim 1.0$ s for a concentration of $\sim 4.1 \times 10^{-3} \mathrm{~mol} \mathrm{~L}^{-1}$.

The addition of background electrolyte at low concentration may induce a stabilising effect against oil exchange between emulsion droplets by acting as a mechanical barrier, hence the observed increase of the time scale of oil exchange to times longer than $\sim 3 \mathrm{~s}$. Upon further increasing electrolyte concentration above $\sim 2.5 \times 10^{-4} \mathrm{~mol} \mathrm{~L}^{-1}$, the corresponding decrease of the surface charge (Table 5.3) may be enough to counter the steric repulsive effect of the $\mathrm{NaCl}$ adsorption layer, hence the observed decrease of $\Delta_{\text {switch }}$ back to values comparable to that measured in the absence of electrolyte.

\subsection{Discussion}

The kinetics of the oil exchange occurring between emulsion droplets of the ternary system toluene/Triton X-100/water have been monitored using PFGNMR. The time scale of oil exchange, determined by the switch between a 
multiexponential and a single exponential decay of the echo attenuation as a function of gradient strength and defined as $\Delta_{\text {switch }}$, spanned from $\sim 300 \mathrm{~ms}$ to $\sim 3 \mathrm{~s}$ depending on oil concentration. In the literature, several workers have measured the time scale of oil exchange occurring between microemulsion droplets ( $\sim$ to 30 nm diameter) using mainly fluorescence quenching experiments, i.e. a probe and a fluorescence quencher are confined within droplets of separately prepared microemulsions and the rate-determining step of the fluorescence quenching is the exchange between droplets. They report oil exchange arising from the reversible coalescence of the microemulsion droplets occurring with time scales spanning from a few microseconds to a few milliseconds for both $\mathrm{O} / \mathrm{W}$ microemulsions stabilised by nonionic surfactants [91, 99] and W/O microemulsions stabilised by ionic surfactants [80]. Similar time scales have been experimentally determined considering an oil exchange between microemulsion droplets via oil permeation upon droplet collision [100].

In kinetically stabilised emulsion systems, Taisne and Cabane probed an oil exchange following a temperature quench leading to droplet growth and occurring via oil permeation upon droplet collision on the timescale of minutes [89]. The time constant of the exchange spanned from $\sim 2 \mathrm{~min}$ at $26^{\circ} \mathrm{C}$ to $\sim 20 \mathrm{~min}$ at $20^{\circ} \mathrm{C}$ (oil volume fraction of 0.2). Thus, the range of time scales of the oil exchange probed in our toluene/Triton X-100/water emulsion system is consistent with that found in the literature, i.e. oil exchange occurs more slowly in emulsions than in microemulsions due to a larger mean droplet size that leads to a slower mean droplet motion based on the Stokes-Einstein equation (Eq. 3.4). To date, the time scale of oil exchange occurring in an emulsion system via droplet contact (oil permeation upon collision or reversible coalescence of the droplets) with no concomitant droplet growth has not been reported.

Furthermore, the effect of droplet size on the rate of oil exchange was also evidenced in our system upon varying toluene concentration. The analysis of the diffusion spectrum and the SLS data both confirmed that the volume fraction of droplets between $\sim 0.5$ and $3 \mu \mathrm{m}$ increased above $\sim 55 \mathrm{wt} \%$ leading to a larger motional averaging value of the oil diffusion and a much longer time scale of the oil exchange $\left(\Delta_{\text {switch }}\right)$. In addition to droplet size, the time scale of oil exchange depends on the mean interdroplet spacing as the frequency of collisions increases 
with droplet concentration leading to faster kinetics of the oil exchange process [91, 94, 101]. In our emulsion system, the increase of the droplet collision frequency was countered by the modification of the properties of the interfacial domain with respect to oil exchange upon increasing droplet concentration as evidenced by the motional averaging of the oil diffusion being independent of oil concentration between 5 and $~ 55$ wt $\%$.

The properties of the interfacial thin film such as the surfactant rigidity, the surfactant chain length, the degree of surfactant packing at the oil/water interface or the presence of adsorbed ions at the interface are of fundamental importance in defining the time scale of oil exchange. The surfactant molecular bending elasticity is proportional to the activation energy of hole nucleation [102], which is a rate-determining step for oil exchange occurring via either reversible coalescence of the droplets or oil permeation upon droplet collision [78, 90]. Therefore, it is expected that the rate of oil exchange is a decreasing function of the surfactant molecular bending elasticity as determined experimentally by Fletcher and Horsup [94]. The surfactant chain length significantly affects the rate of surfactant desorption upon hole formation [103], hence the rate of oil exchange should be a decreasing function of surfactant chain length as confirmed experimentally by Jada et al. [92].

The addition of a cosurfactant such as benzyl alcohol or hexanol residing at the oil/water interface may interfere with the stabilising interactions between surfactant molecules (e.g. AOT), hence increasing the rate of exchange [100]. The presence of cosurfactant can also alter the rate of exchange by swelling the surfactant head group more than the surfactant tail favouring a less negative (W/O emulsions) or more positive (O/W emulsions) spontaneous curvature of the surfactant film, hence facilitating (W/O emulsions) or hindering (O/W emulsions) oil exchange since hole formation is always coupled with the surfactant molecules being forced to adopt a curvature of opposite sign around the hole [80]. In O/W emulsions this means the surfactant molecules must adopt a local highly negative curvature around the hole as the tail region is locally expanded and the head region is locally compressed (Figure 5.12). 


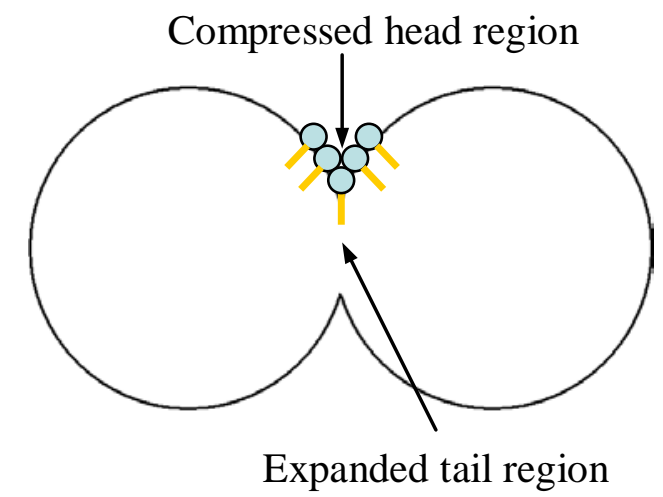

Figure 5.12. Hole nucleation in an $\mathrm{O} / \mathrm{W}$ emulsion.

Upon varying oil concentration from 5 to $\sim 55 \mathrm{wt} \%$ in the toluene/Triton $\mathrm{X}$ 100/water system while maintaining the same droplet size distribution and a constant oil-to-surfactant weight ratio, the degree of surfactant packing at the toluene/water interface, other controlling parameters (energy input, method of formulation, temperature and nature of the constituents) being equal, is believed to be an increasing function of oil concentration, leading to a more rigid interface via enhanced steric stabilization. This accounts for the time scale of oil exchange ( $\left.\Delta_{\text {switch }}\right)$ being an increasing function of oil concentration despite the droplets being closer to each other.

The addition of salt ions causes the dehydration of the surfactant polar head groups in either $\mathrm{O} / \mathrm{W}$ and $\mathrm{W} / \mathrm{O}$ emulsions, resulting in the decrease of both the effective head group volume and the distance between the head groups [90]. The decrease of the spacing between the surfactant head groups while maintaining the same distance between the surfactant tails leads to a less positive equilibrium curvature of the surfactant layer [104]. According to Kabalnov and Wennerström [78], the rate of hole nucleation in $\mathrm{O} / \mathrm{W}$ emulsions is proportional to the energy barrier for the formation of local negative curvatures. As such, the addition of salt in toluene/Triton X-100/water emulsions decreases the energy cost for hole nucleation, which should facilitate oil exchange. Several investigations indicate that the rate of oil exchange decreases upon increasing the ionic strength in both $\mathrm{O} / \mathrm{W}$ and W/O emulsion systems $[86,89,100]$. The decrease of the energy barrier for oil exchange may be countered by the steric barrier formed by the salt ions 
bound to the surfactant thin film. These steric repulsive forces augment the rigidity of the interfacial domain with regard to oil transfer.

On the other hand, our PFG-NMR data upon varying the observation time, $\Delta$, are similar to those obtained in porous systems where a fluid is able to flow within interconnected pores. When restricted diffusion is probed, the plot of $\mathrm{D}(\mathrm{t}) / \mathrm{D}_{0}$, where $\mathrm{D}(\mathrm{t})$ is the time dependent diffusion coefficient of the fluid and $\mathrm{D}_{0}$ is the bulk diffusion coefficient, is a decreasing function of the observation time, $\Delta$, [105]. At short $\Delta$ values the diffusion coefficient is proportional to the surface-tovolume ratio of the pore space $[106,107]$ whereas at long observation times, the plot of $\mathrm{D}(\mathrm{t}) / \mathrm{D}_{0}$ versus $\Delta$ follows an asymptote which is proportional to the inverse of the system tortuosity (Figure 5.13) [108-111].

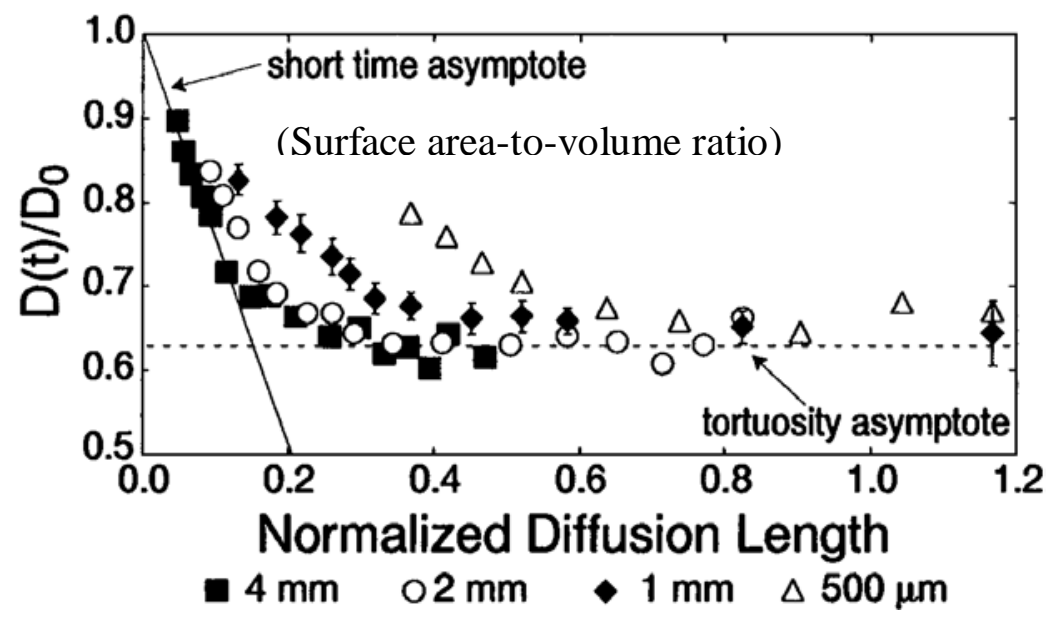

Figure 5.13. Time-dependent normalised diffusion coefficient for thermally polarised xenon gas imbibed in randomly packed spherical glass beads taken from reference [111]. Beads have uniform diameters, $b$, of $0.5,1,2$ and $4 \mathrm{~mm}$. The gas diffusion coefficient in the porous medium is normalised to the gas bulk diffusion coefficient $\mathbf{D}_{\mathbf{0}}$. The normalised diffusion length is defined as $(1 / b)\left(D_{0} t\right)^{1 / 2}$.

In toluene/Triton X-100/water emulsions, a similar trend is observed for the diffusion of the largest droplets which is restricted by the presence of other droplets for observation times comprised between $\sim 20$ and $~ 50$ ms (Figure 5.2 and Figure 5.3). In Figure 5.14 is plotted the apparent diffusion coefficient associated with the peak corresponding to the slowest diffusion of the spectrum (maximum of peak 1, Figure 5.2) as a function of the square root of the NMR observation time, $\Delta$, for a $40 \mathrm{wt} \%$ toluene emulsion. The range of observation 
times when restricted diffusion is detected is too limited (instrument limitations on both the short and long sides) to explore this fully. However, the long time limit lies around $D=1.0 \times 10^{-13} \mathrm{~m}^{2} \mathrm{~s}^{-1}$, which is two orders of magnitude smaller than the measured motional averaging of the oil diffusion $\left(D=(1.5 \pm 0.3) \times 10^{-11}\right.$ $\mathrm{m}^{2} \mathrm{~s}^{-1}$ for a $40 \mathrm{wt} \%$ toluene sample). Therefore, the motional averaging detected in our system cannot be related to a porous medium effect.

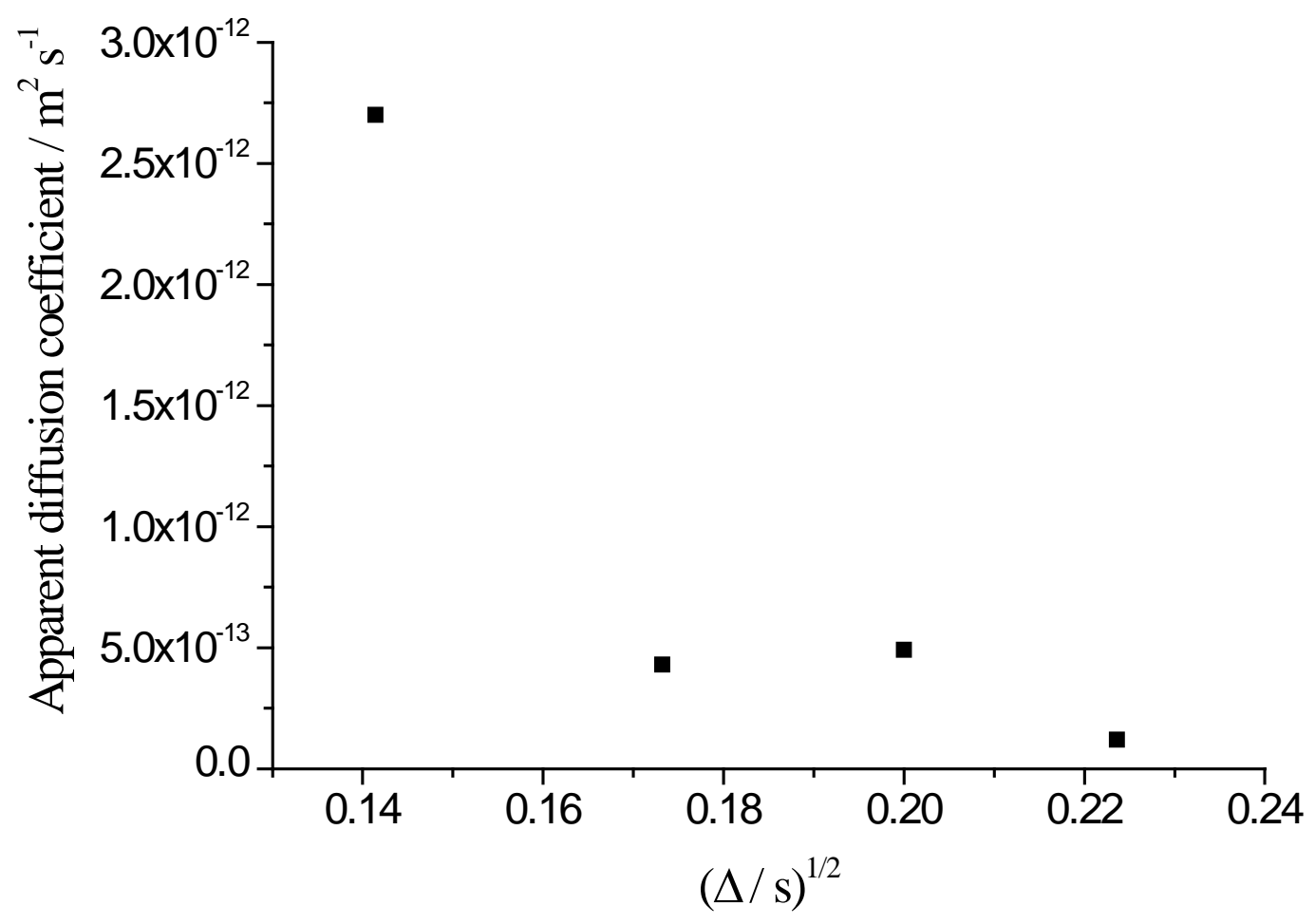

Figure 5.14. Apparent diffusion coefficient associated with the restricted diffusion of the largest droplets as a function of the NMR observation time, $\Delta$, for a $40 \mathrm{wt} \%$ toluene emulsion.

\subsection{Conclusion}

In conclusion, the time scale of the oil exchange occurring between droplets of toluene/Triton X-100/water emulsions was determined using PFG-NMR,

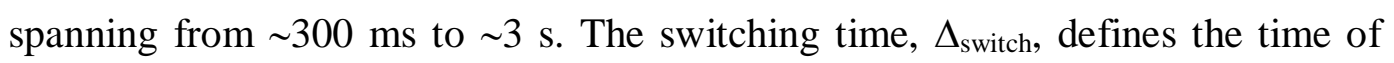
the switch between a multiexponential decay, where the emulsion polydispersity is probed, and a single exponential decay of the echo attenuation as a function of the gradient strength, when motional averaging of the oil diffusion is detected, i.e. oil exchange occurs on a time scale faster than $\Delta_{\text {switch. }}$.

Analysis of the diffusion spectrum extracted from the multiexponential decay attenuation data via an inverse Laplace transform suggests the presence of toluene 
molecules and oil swollen micelles (10 - $30 \mathrm{~nm}$ diameter) freely diffusing in the aqueous continuous phase. Also, both the diffusion spectrum and the SLS data indicate an increase of the volume fraction of droplets between $\sim 0.5$ and $3 \mu \mathrm{m}$ diameter upon increasing toluene concentration above $~ 55 \mathrm{wt} \%$. This results in the corresponding increase of the motional averaging value of the oil diffusion, which is proportional to the average distance travelled by oil molecules during oil exchange, as well as a steep increase of the time scale of oil exchange.

Moreover, the effect of the properties of the interfacial domain on the kinetics of oil exchange was probed upon varying toluene concentration from 5 to 55 wt $\%$ and through the addition of background electrolyte in the continuous phase before emulsification. The increase of the surfactant packing at the oil/water interface upon increasing toluene concentration from 5 to 55 wt $\%$ accounts for the concomittant increase of the time scale of oil exchange $\left(\Delta_{\text {switch }}\right)$ since the droplet size and the oil-to-surfactant weight ratio remain unchanged whereas the droplets become more concentrated. The variation of the interfacial domain rigidity upon increasing oil content from 5 to $\sim 55 \mathrm{wt} \%$ is consistent with the previous identification of three concentration domains including a low-oil content dispersed droplet O/W emulsion between 5 and $~ 25 \mathrm{wt} \%$ oil, a transition domain between $\sim 25$ and $\sim 35 \mathrm{wt} \%$ oil and a high-oil content dispersed droplet O/W emulsion between $\sim 35$ and $~ 55$ wt $\%$ oil [41].

Finally, the time scale of oil exchange could be manipulated by the addition of a background electrolyte such as $\mathrm{NaCl}$. The binding of the $\mathrm{Na}^{+}$ions to the surfactant layer resulted in the decrease of the rate of exchange due the increase of the steric repulsive forces. 


\section{Chapter 6 Ageing of toluene/Triton X- 100/water emulsions}

\subsection{Introduction}

As kinetically stabilised systems, the microstructure and dynamics of emulsions change with time. For any given system, droplets grow at a rate which depends on the composition of the constituents, the initial droplet size distribution and the droplet concentration as well as the properties of the interfacial domain controlling the mutual interactions between oil, emulsifier and water. Once a critical mean droplet size is reached upon ageing, emulsions macroscopically phase separate into a more concentrated emulsion (an $\mathrm{O} / \mathrm{W}$ for the toluene/Triton X-100/water system), an expelled oil phase on the top and an expelled aqueous phase at the bottom. This continues until total phase separation into isolated oil and water domains, representing the equilibrium state of the system, occurs.

The investigation of emulsion ageing including how the droplet size distribution, the rate of droplet growth and the dynamics of the oil phase vary with time and oil concentration gives us an insight into the characteristics of the interfacial domain in toluene/Triton X-100/water emulsions.

In a system destabilizing mainly via coalescence and where the properties of the surfactant layer as well as the droplet size remain unchanged upon increasing oil concentration, the rate of droplet growth is expected to be an increasing function of the droplet collision frequency. Based on the macroscopic phase separation, light scattering and PFG-NMR ageing data of toluene/Triton X-100/water emulsions, the rate of coalescence is reported to be a decreasing function of the droplet encounter frequency. One way to explain this outcome is to consider the augmentation of the surfactant packing at the oil/water interface upon increasing oil content. 


\subsection{Macroscopic phase separation}

In the previous chapter, the existence of four concentration domains was highlighted, including a low-oil content region between 5 and 25 wt\% toluene, a high-oil content region between $\sim 35$ and $\sim 55 \mathrm{wt} \%$ toluene, a transition region between $\sim 25$ and $\sim 35 \mathrm{wt} \%$ oil and a closed-cell foam region between $\sim 55$ and 70 wt\% [41]. Macroscopic phase separation experiments were performed with respect to the aqueous phase containing water as well as toluene molecules and toluene swollen micelles dissolved in the continuous phase. The phase separation data upon increasing toluene concentration from 5 to $70 \mathrm{wt} \%$ are shown in Figure 6.1 (not all concentrations are shown for clarity).

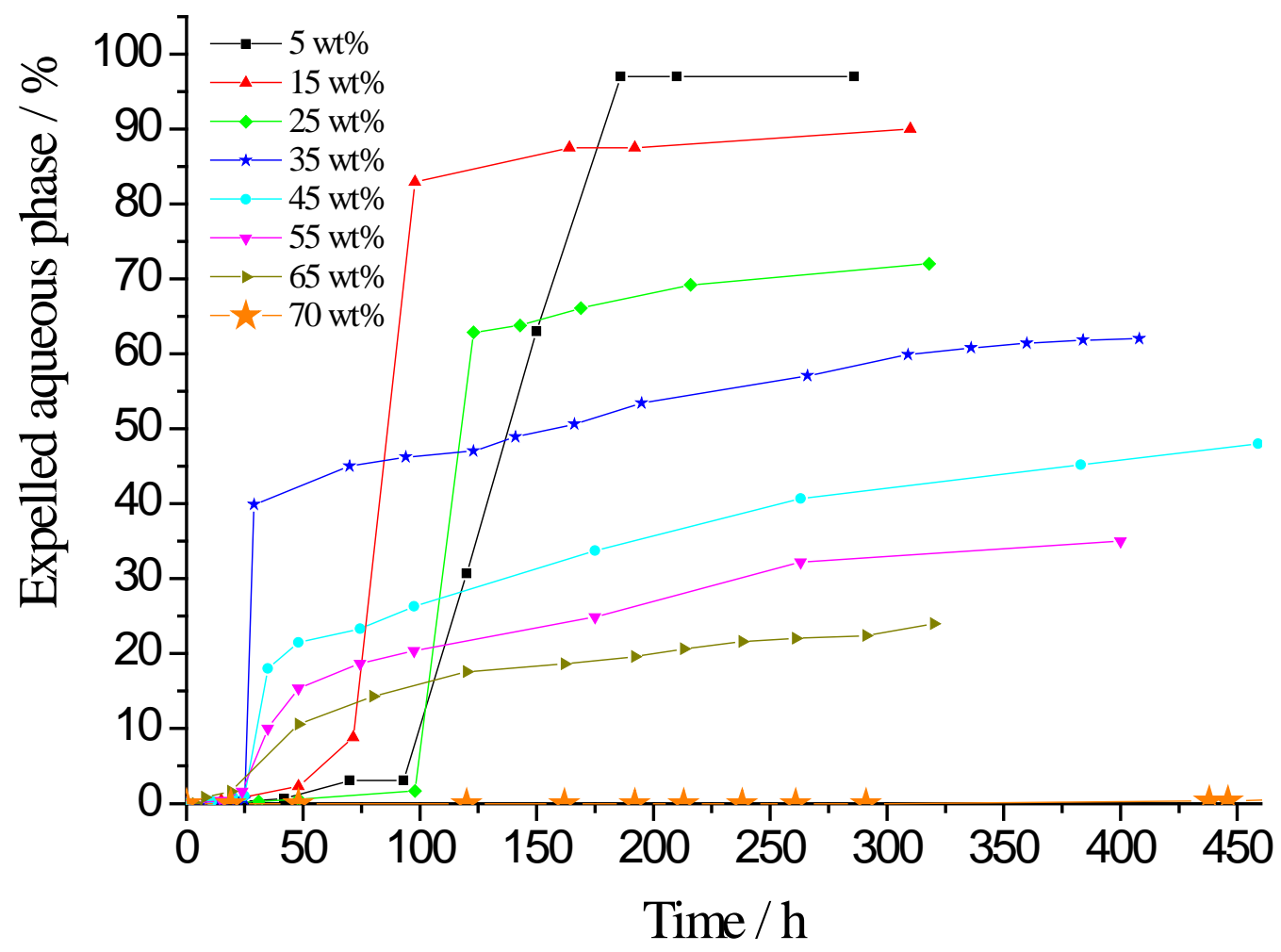

Figure 6.1. Amount of aqueous phase expelled as a function of time for toluene/Triton $\mathrm{X}$ 100/water emulsions prepared with toluene between 5 and $70 \mathrm{wt} \%$.

For the highest toluene concentration of $70 \mathrm{wt} \%$, no macroscopic phase separation is detected until $\sim 18$ days (432 h, $0.3 \%$ water is expelled) after emulsification (Figure 6.1). This is due to the sample's low water volume fraction (16.5\%), the sample's gel-like characteristics, which keeps the water phase 
trapped and the high stability of the interfacial domain due to the reduction of the Laplace pressure and the formation of Plateau borders and indices [40].

The four separate emulsion domains are readily distinguished in this experiment. Three groups corresponding to the low-oil content, high-oil content and transition regions can be distinguished with respect to

1) the time corresponding to the beginning of the phase separation,

2) the duration of the initial fast phase separation, i.e. that corresponding to a steep increase in the amount of expelled phase is observed,

3) the time $t_{\text {static }}$ at which the phase separation considerably slows down, i.e. when the amount of expelled phase remains reasonably constant and

4) the rate of macroscopic phase separation.

Given the droplet size distribution of toluene/Triton X-100/water emulsions remains unchanged from 5 to $55 \mathrm{wt} \%$ toluene (Figure 4.1), the four parameters mentioned above mainly depend on the droplet concentration and the surfactant packing at the oil/water interface. Above $60 \mathrm{wt} \%$ toluene, the increase of the mean droplet size (Figure 4.1) is expected to accelerate the destabilization process as larger droplets grow faster than smaller droplets due to a larger surface contact area with their neighbours.

For the subset of emulsions prepared with toluene between 40 and $65 \mathrm{wt} \%$, the plot of the amount of expelled aqueous phase versus time is shown in Figure 6.2 and the corresponding characteristic times are given in Table 6.1. 


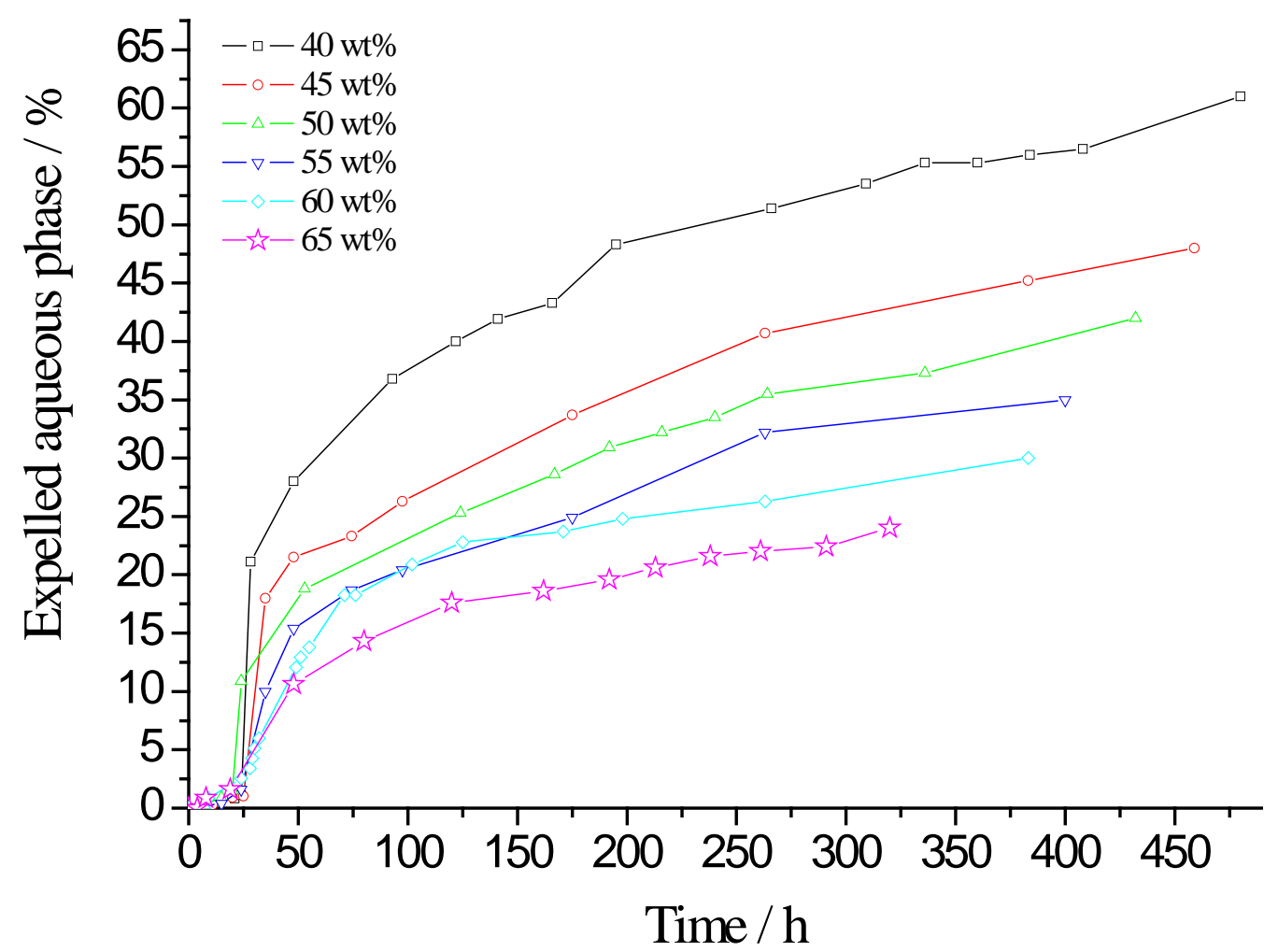

Figure 6.2. Amount of aqueous phase expelled as a function of time for toluene/Triton X100/water emulsions prepared with toluene between 40 and 65 wt\%.

\begin{tabular}{|c|c|c|c|}
\hline $\begin{array}{c}\text { Toluene } \\
\text { concentration / } \\
\text { wt\% }\end{array}$ & $\begin{array}{c}t_{\text {start of phase separation }} \\
( \pm 2) / \mathrm{h}\end{array}$ & $\mathrm{t}_{\text {static }}( \pm 2) / \mathrm{h}$ & $\begin{array}{c}\text { Extent of phase } \\
\text { separation at } \mathrm{t}_{\text {static }} / \\
\%\end{array}$ \\
\hline 40 & 10 & 480 & 61 \\
\hline 45 & 11 & 460 & 48 \\
\hline 50 & 7 & 432 & 42 \\
\hline 55 & 10 & 400 & 35 \\
\hline 60 & 9 & 383 & 30 \\
\hline 65 & 8 & 320 & 24 \\
\hline
\end{tabular}

Table 6.1. Characteristic times and amount of expelled aqueous phase during the macroscopic phase separation process for emulsions between 40 and $65 \mathrm{wt} \%$ toluene.

The time, $t_{\text {static}}$, taken to reach a nearly constant amount of expelled aqueous phase, i.e. when most of the phase separation process has proceeded, is a decreasing function of oil concentration for samples within the high-oil content domain in addition to those emulsions containing 60 to 65 wt\% toluene (Table 6.1). This trend is expected since the time taken to reach the same extent of phase 
separation associated with a specific mean droplet size is proportional to droplet collision frequency or droplet concentration.

In contrast, both the time at which macroscopic phase separation is detected, spanning from $\sim 7$ to $\sim 11 \mathrm{~h}$ post emulsification (Table 6.1 ), and the time of the initial rapid phase separation (steep increase of the amount of expelled aqueous phase around 24 h, Figure 6.2) are independent of oil concentration. However the critical droplet size activating macroscopic phase separation is expected to be reached more rapidly upon decreasing the interdroplet spacing and increasing droplet concentration in a system where the oil-to-surfactant weight ratio is maintained, i.e. the interfacial domain properties are equal. However, this observation is in agreement with the data presented in chapter 5 considering the time scale of the oil exchange process (NMR switching time, $\Delta_{\text {switch }}$ ) between emulsion droplets upon increasing oil concentration (Figure 5.11). The decrease of the rate of oil exchange upon increasing droplet concentration suggests that the rigidity of the surfactant thin film, imposed by the surfactant packing at the oil/water interface, rises with oil content despite the constant oil-to-surfactant weight ratio. The fact that macroscopic phase separation, induced by a critical mean droplet size, occurs at a time that is independent, as opposed to being a decreasing function, of oil concentration from 40 to $65 \mathrm{wt} \%$ toluene further supports this conclusion.

The phase separation data were normalised with respect to the extent of expelled aqueous phase at $t_{\text {static }}$ (Figure 6.3). All data conform to a single curve indicating that the rate of phase separation is independent of oil concentration from 40 to 65 wt $\%$ as opposed to being proportional to droplet concentration and to the initial mean droplet size (the mean droplet size increases by $60 \%$ from $60 \mathrm{wt} \%$ toluene, Figure 4.1). Similarly, the observed trend of the rate of phase separation as a function of oil content corroborates the idea of an increase of the surfactant packing with oil concentration within the high-oil content region. 


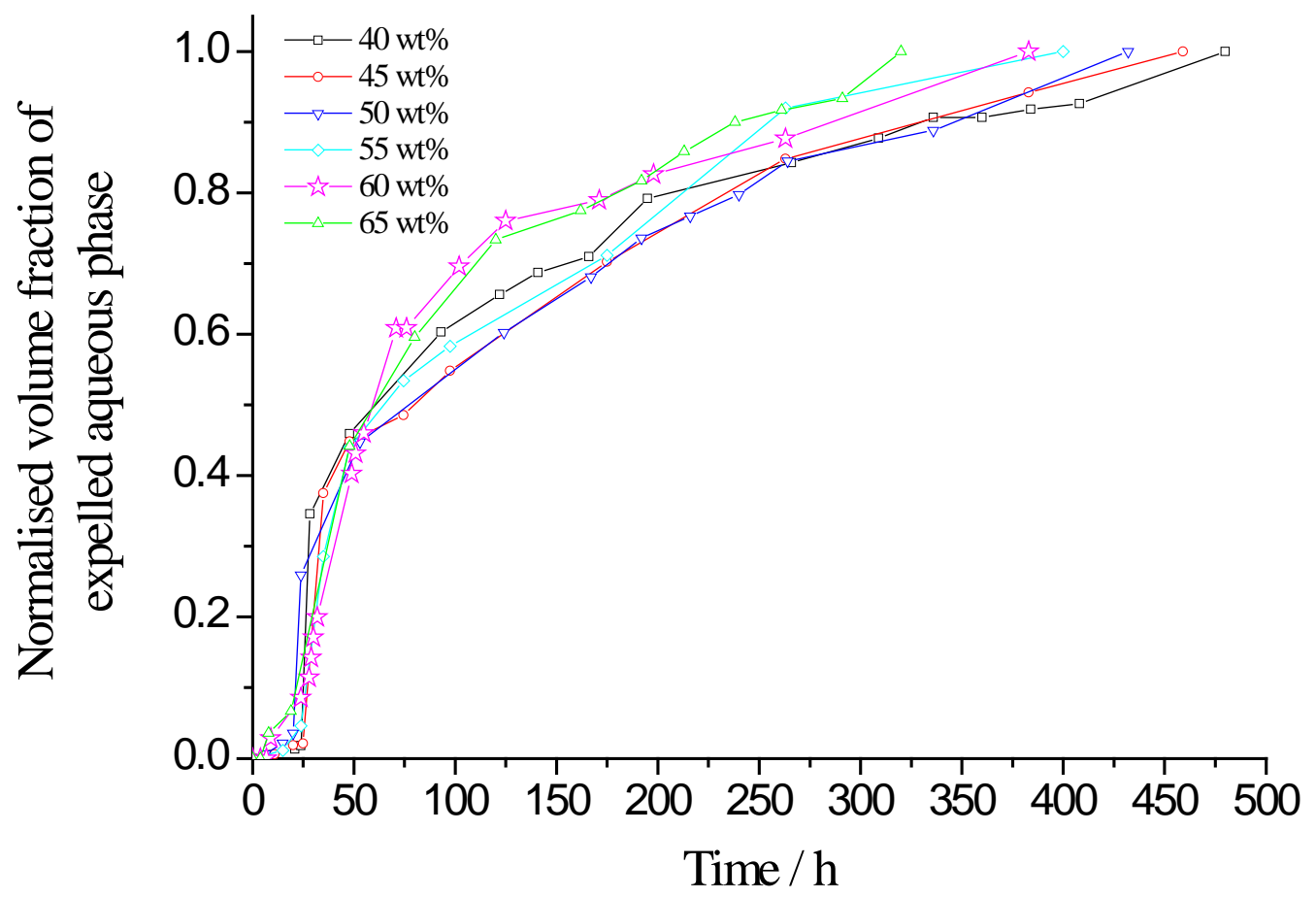

Figure 6.3. Volume fraction of expelled aqueous phase normalised to the limiting value measured at $t_{\text {static }}$ as a function of time for toluene/Triton $\mathrm{X}-100 /$ water emulsions prepared with toluene between 40 and 65 wt\%.

For the emulsions of the low-oil content region, between 5 and $~ 20 \mathrm{wt} \%$ toluene, the macroscopic phase separation data normalized to the extent of phase separation measured at $t_{\text {static }}$ are shown in Figure 6.4 and the associated characteristic times are given in Table 6.2.

Macroscopic phase separation is initiated between $\sim 15$ to $\sim 24 \mathrm{~h}$ with the time of the initial rapid phase separation being in the range of $\sim 47$ to $\sim 93 \mathrm{~h}$ after emulsion formation (Table 6.2). These times are longer than those of the high-oil content region (Table 6.1), as expected in more diluted samples due to the larger average interdroplet spacing and the concomitant decrease of the droplet encounter frequency.

In contrast to the high-oil content region, the time at which macroscopic phase separation begins and the time of the initial rapid phase separation are both decreasing functions of oil concentration (Table 6.2) and the rate of phase separation is a slightly rising function of oil concentration (Figure 6.4) within the low-oil content domain. This indicates that interdroplet distance predominantly contributes to the rate of phase separation in the early stages of destabilisation. 
Once the initial rapid phase separation has ended and the mean droplet spacing has significantly decreased, the time, $\mathrm{t}_{\text {static }}$, to reach a nearly constant amount of expelled aqueous phase, i.e. the time for complete phase separation to occur, increases with oil content (Table 6.2). This suggests that once the macroscopic phase separation is at an advanced stage, the properties of the surfactant layer overcome interdroplet distance effects. In the literature, the same two regimes have been identified for systems principally destabilizing via coalescence, i.e. at low volume fraction of dispersed phase the droplet encounter frequency is the rate determining step of emulsion destabilization whereas at higher droplet concentration the properties of the film predominate $[112,113]$.

Although the times at which phase separation begins and those of the fast phase separation are longer in the low oil content domain compared to the high oil content region due to droplets being further apart from each other in the initial stage of destabilisation, the times for complete phase separation to occur spanning from $\sim 186$ to $\sim 336 \mathrm{~h}$ at low oil concentration (Table 6.2) are shorter than at high oil content ( $\sim 320$ to $\sim 480 \mathrm{~h}$, Table 6.1$)$, which is consistent with the enhancement of the interface coverage upon increasing oil concentration. 


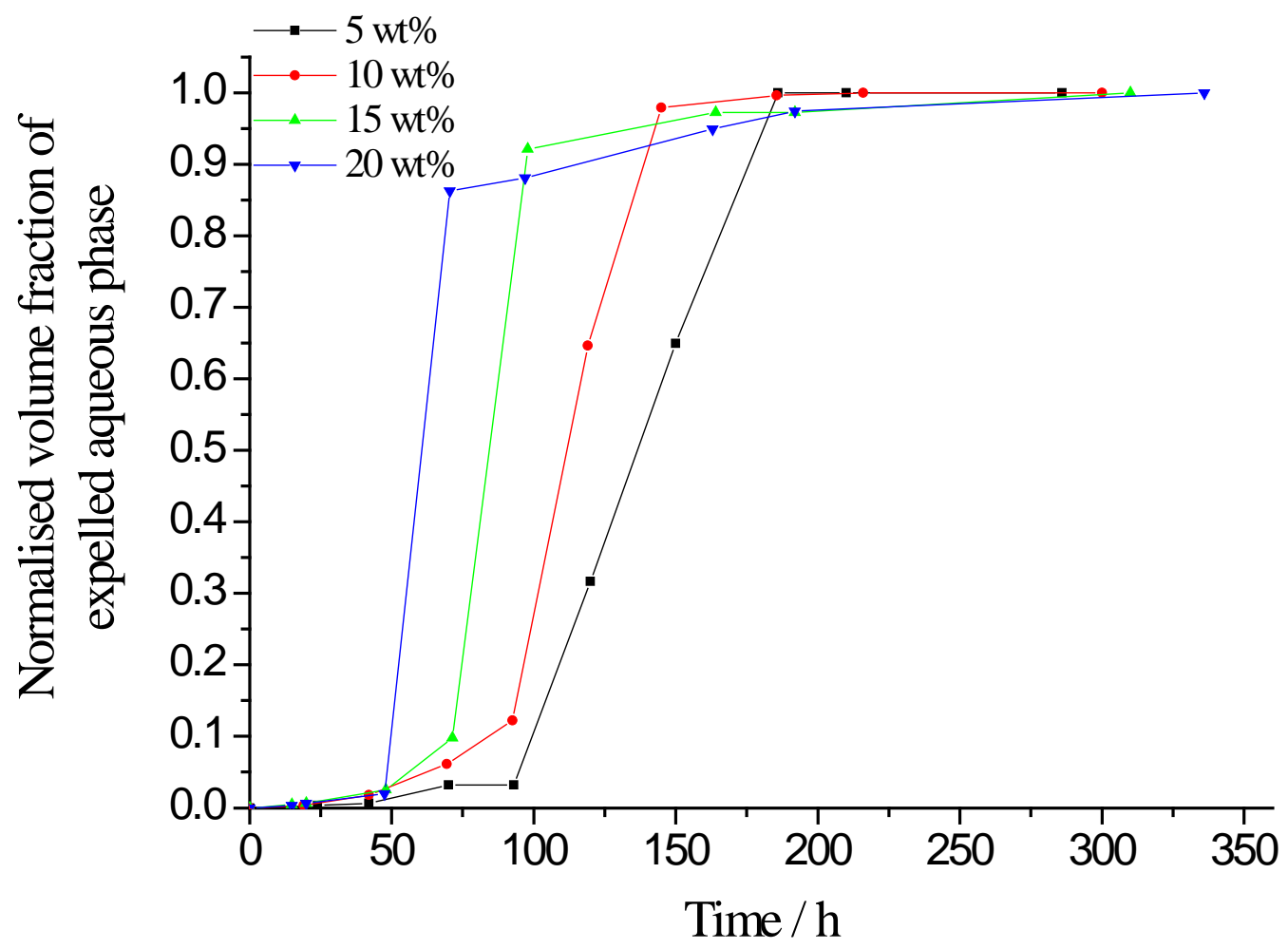

Figure 6.4. Volume fraction of expelled aqueous phase normalised to the limiting value measured at $t_{\text {static }}$ as a function of time for toluene/Triton $\mathrm{X}-100 /$ water emulsions prepared with toluene between 5 and $20 \mathrm{wt} \%$.

\begin{tabular}{|c|c|c|c|c|}
\hline $\begin{array}{c}\text { Oil } \\
\text { concentration } \\
/ \mathrm{wt} \%\end{array}$ & $\begin{array}{c}\mathrm{t}_{\text {start of phase }} \\
\text { separation }( \pm 2) / \\
\mathrm{h}\end{array}$ & $\begin{array}{c}\mathrm{t}_{\text {initial fast phase }} \\
\text { separation }( \pm 2) \\
/ \mathrm{h}\end{array}$ & $\mathrm{t}_{\text {static }}( \pm 2) / \mathrm{h}$ & $\begin{array}{c}\text { Extent of phase } \\
\text { separation at } \\
\mathrm{t}_{\text {static }} / \%\end{array}$ \\
\hline 5 & 24 & 93 & 186 & 97 \\
\hline 10 & 19 & 80 & 216 & 95 \\
\hline 15 & 15 & 71 & 310 & 90 \\
\hline 20 & 15 & 47 & 336 & 85 \\
\hline
\end{tabular}

Table 6.2. Characteristic times of the phase separation process and maximum expelled aqueous phase for emulsions between 5 and $20 \mathrm{wt} \%$ toluene.

Finally, emulsions belonging to the transition concentration domain, between $\sim 25$ and $~ 35 \mathrm{wt} \%$ toluene, underline a phase separation behaviour similar to that of the low-oil content domain, i.e. the time at which destabilization starts and the time scale of the initial fast phase separation are concentration dependent (Figure 6.5 and Table 6.3). 


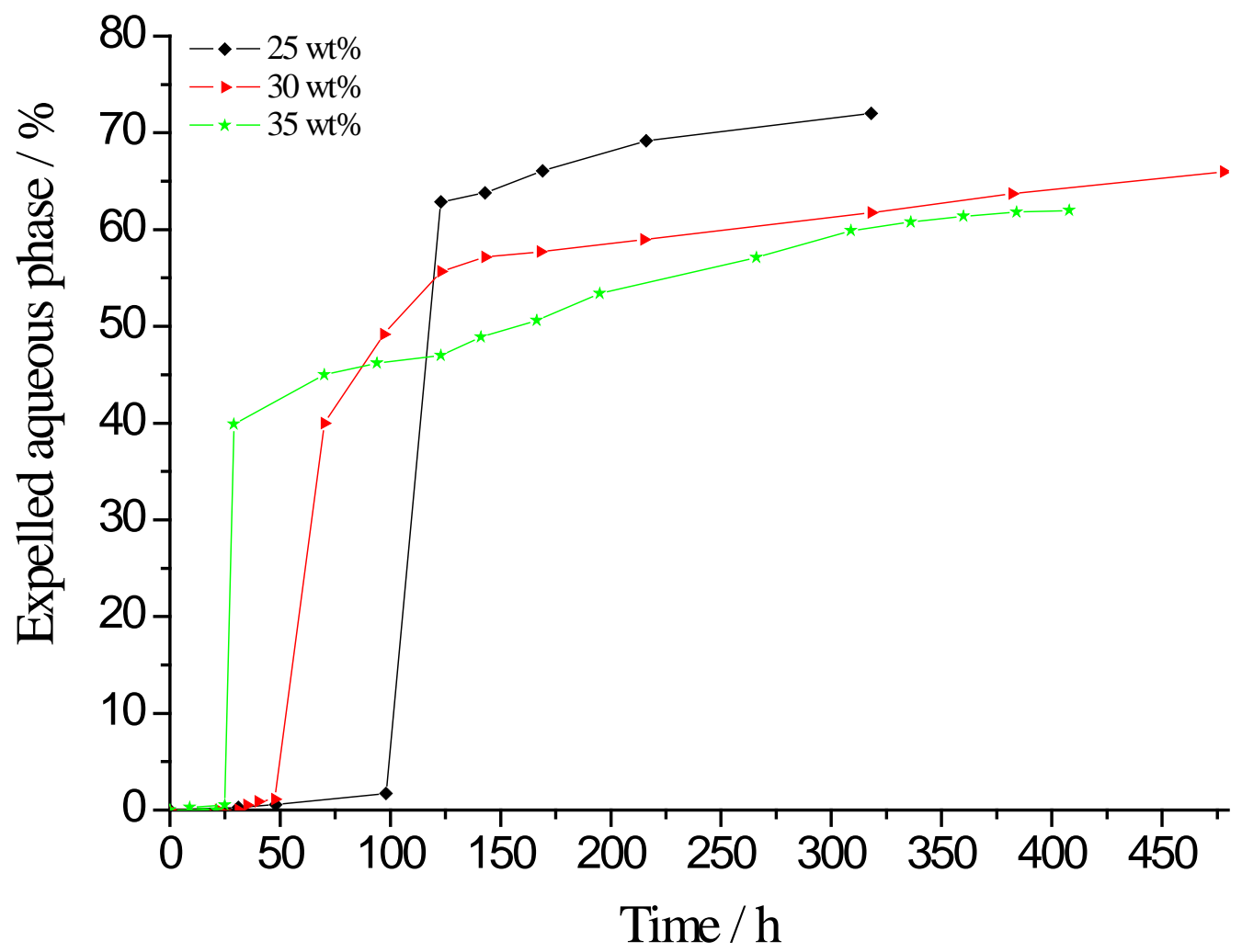

Figure 6.5. Percentage of expelled aqueous phase as a function of time for toluene/Triton $\mathrm{X}$ 100/water emulsions prepared with toluene between 25 and $35 \mathrm{wt} \%$.

\begin{tabular}{|c|c|c|c|c|}
\hline $\begin{array}{c}\text { Oil } \\
\text { concentration } \\
/ \mathrm{wt} \%\end{array}$ & $\begin{array}{c}\mathrm{t}_{\text {start of phase }} \\
\text { separation }( \pm 2) / \\
\mathrm{h}\end{array}$ & $\begin{array}{c}\mathrm{t}_{\text {initial fast phase }} \\
\text { separation }( \pm 2) \\
/ \mathrm{h}\end{array}$ & $\mathrm{t}_{\text {static }}( \pm 2) / \mathrm{h}$ & $\begin{array}{c}\text { Extent of phase } \\
\text { separation at } \\
\mathrm{t}_{\text {static }} / \%\end{array}$ \\
\hline 25 & 31 & 98 & 318 & 72 \\
\hline 30 & 35 & 47 & 478 & 66 \\
\hline 35 & 9 & 25 & 408 & 62 \\
\hline
\end{tabular}

Table 6.3. Characteristic times of the phase separation process and maximum expelled aqueous phase for emulsions between 25 and $35 \mathrm{wt} \%$ toluene.

Destabilisation within samples prepared with toluene between 5 to 35 wt\% are distinguished from the high-oil content and the closed-cell foam regions. Two emulsion layers are evident upon macroscopic phase separation at low oil content. A top emulsion layer is separated from a more transparent bottom emulsion layer via a clear interface (Figure 6.6). 


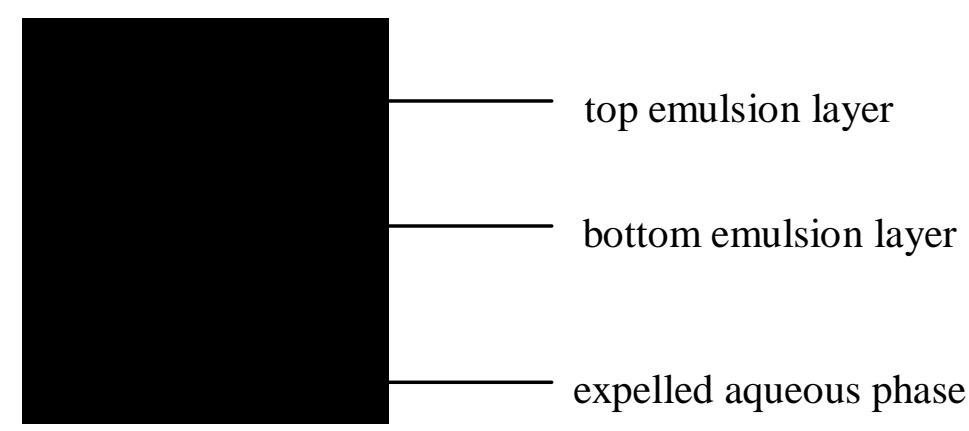

Figure 6.6. Emulsion prepared with $15 \mathrm{wt} \%$ toluene observed $\sim 48 \mathrm{~h}$ post emulsification. This picture is representative of emulsion samples between 5 and $35 \mathrm{wt} \%$ toluene.

The two emulsion layers could be separated through the use of a separating funnel. Once separated, the size distributions were determined using SLS (Figure 6.7). The two droplet size distributions indicate the presence of a droplet size gradient leading to the formation of two emulsion layers on top of each other. The bottom emulsion layer contains the smallest droplets measuring between $35 \mathrm{~nm}$ and $3 \mu \mathrm{m}$ diameter, most being smaller than $\sim 0.4 \mu \mathrm{m}$, whereas the top emulsion layer comprises the full range of droplet sizes, i.e. between $35 \mathrm{~nm}$ and $20 \mu \mathrm{m}$. During the phase separation process, as the mean droplet size increases, the bottom emulsion layer progressively disappears while the extent of the top emulsion layer in the experimental tube remains constant.

The fact that a droplet size gradient is not seen in the more concentrated emulsions between 40 and $70 \mathrm{wt} \%$ and that the time required for this droplet size gradient to be observed at low oil content is a decreasing function of oil concentration ( $\sim 15$ to $\sim 70 \mathrm{~h}$, Table 6.4 ) confirms that the time to reach the same extent of phase separation or mean droplet size depends on droplet concentration in the early stages of destabilization for the low-oil content region. 


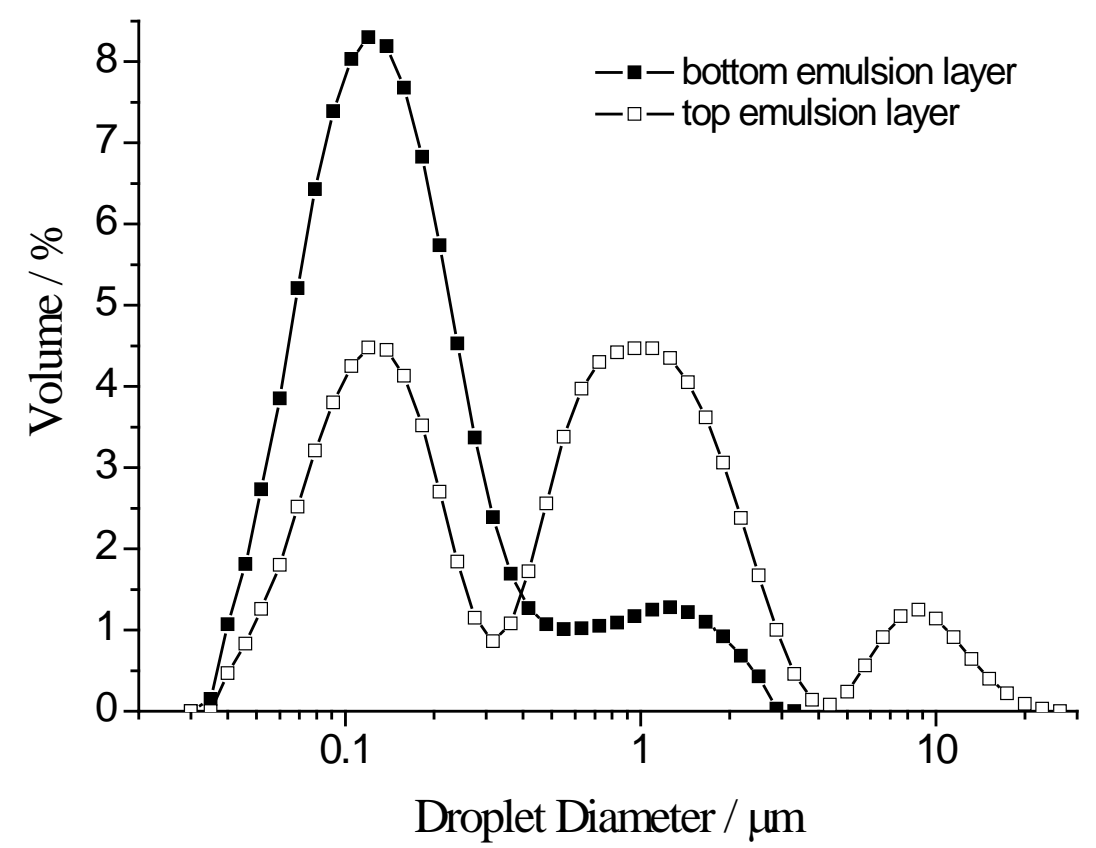

Figure 6.7. Droplet size distribution of the two emulsion layers observed after $\sim 40 \mathrm{~h}$ for a phase separated emulsion prepared with $20 \mathrm{wt} \%$ toluene and measured separately using SLS.

\begin{tabular}{|c|c|}
\hline Oil concentration / wt\% & $\mathrm{t}_{\text {droplet size gradient }} / \mathrm{h}$ \\
\hline 10 & 70 \\
\hline 15 & 48 \\
\hline 20 & 40 \\
\hline 25 & 24 \\
\hline 35 & 15 \\
\hline
\end{tabular}

Table 6.4. Time of visualisation of a droplet size gradient upon varying oil concentration between 10 and $35 \mathrm{wt} \%$.

\subsection{Destabilisation mechanisms}

In order to determine the nature of the destabilisation mechanisms occurring in the four concentration domains of the toluene/Triton X-100/water system, emulsions prepared with 15, 25, 40 and 65 wt\% toluene, representative of the low-oil content, transition, high-oil content and closed cell foam domains, respectively, were analysed using SLS to measure the droplet size distribution over a period of 5 days. 
In Figure 6.8 is shown the droplet size distribution variation with time for a 40 wt\% toluene emulsion. The distribution and the corresponding variation is representative of all four concentration regions, i.e. the changes of the droplet size distribution with time are independent of oil concentration, only the time scale at which the transitions occur are concentration dependent.

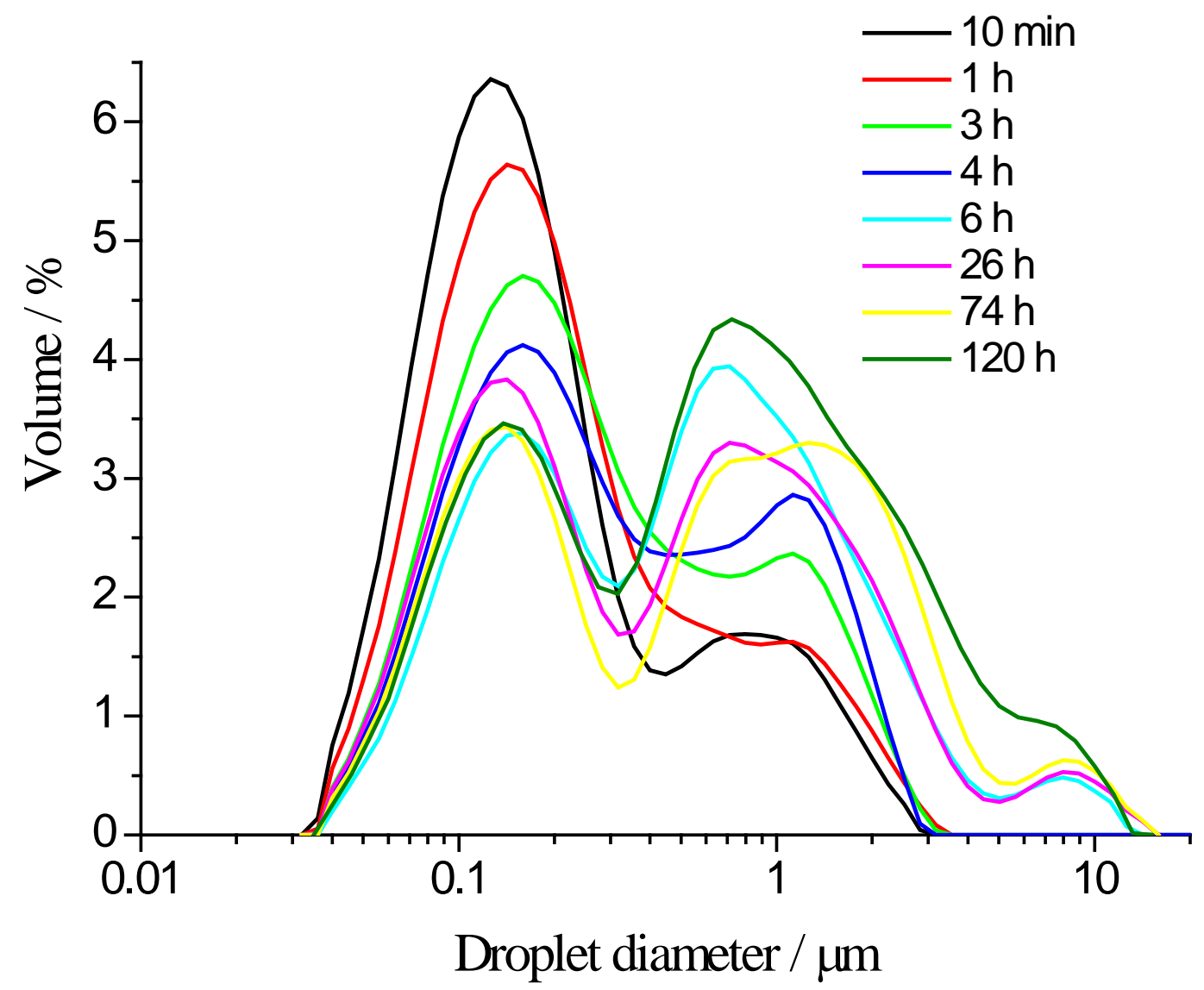

Figure 6.8. Droplet size distribution of a $40 \mathrm{wt} \%$ toluene emulsion measured as a function of time over 5 days using SLS. This size distribution is representative of emulsions between 5 and $70 \mathrm{wt} \%$ oil.

The droplet size distribution of the toluene/Triton X-100/water emulsions becomes multimodal upon ageing including droplets as large as $16 \mu \mathrm{m}$ being formed (Figure 6.8). For a $40 \mathrm{wt} \%$ toluene emulsion, during the first $\sim 6$ hours following emulsification, oil droplets remain in the same size range as that measured for freshly emulsified samples, i.e. between $35 \mathrm{~nm}$ and $\sim 3 \mu \mathrm{m}$ diameter. During this period, the distribution is bimodal with the increase of the number of droplets measuring $\sim 0.4$ to $\sim 3 \mu \mathrm{m}$ diameter, as seen by the growth of the peak centered at $\sim 1 \mu \mathrm{m}$ being at the expense of the number of droplets between $\sim 35$ 
$\mathrm{nm}$ and $\sim 0.35 \mu \mathrm{m}$ diameter, as observed by the concomitant reduction of the amplitude of the peak centered at $\sim 0.15 \mu \mathrm{m}$.

From $\sim 6$ h onwards a third peak centered at $\sim 8 \mu \mathrm{m}$ including droplets between $\sim 3.5$ and $\sim 16 \mu$ m diameter is detected. Once a critical mean droplet size has been reached, corresponding to the presence of large oil droplets $(\sim 3.5-16 \mu \mathrm{m}$ diameter), the emulsion macroscopically phase separates; for a $40 \mathrm{wt} \%$ toluene emulsion this occurs after $\sim 10 \mathrm{~h}$ (Table 6.1 ). Finally, between $\sim 6$ and $\sim 120 \mathrm{~h}$, the amplitude ratio of the peak centered at $\sim 1 \mu \mathrm{m}$ to the peak centered at $\sim 0.15 \mu \mathrm{m}$ continues to increase coupled with a slight growth of the third peak.

The effect of oil concentration on the time scale of the main transitions observed in the droplet size distribution over time are summarised in Table 6.5. The time at which droplet growth starts, as detected by the variation of the SLS volume-based mean droplet size, spanning from $\sim 15$ to $\sim 45$ min post emulsification, is a slightly decreasing function of oil content, which is consistent with the mean interdroplet distance also being a decreasing function of oil concentration.

While droplet concentration may account for the time scale of the formation of the largest droplets ( 3.5-16 $\mu \mathrm{m}$ diameter) being a decreasing function of oil concentration from $\sim 68$ to $\sim 6 \mathrm{~h}$, it cannot solely explain the much slower growth of these larger droplets in the $15 \mathrm{wt} \%$ toluene sample, i.e. around $\sim 68 \mathrm{~h}$.

On the other hand, the time at which phase separation begins is proportional to the emulsion mean droplet size. Here, the variation of the volume-based mean droplet size with oil content in the early stages of destabilization, e.g. between $\sim 0.50$ and $\sim 0.97 \mu \mathrm{m}$ after $6 \mathrm{~h}$, fits well with the corresponding time at which phase separation starts (Table 6.5.).

\begin{tabular}{|l|c|c|c|c|}
\hline Oil concentration / wt\% & 15 & 25 & 40 & 65 \\
\hline $\mathrm{t}_{\text {droplet growth }( \pm 15) / \mathrm{min}}$ & 45 & 30 & 30 & 15 \\
\hline $\mathrm{t}_{\text {appearance of 3rd peak }(\sim 8 \mathrm{~m})}( \pm 2) / \mathrm{h}$ & 68 & 8 & 6 & 6 \\
\hline $\mathrm{t}_{\text {macroscopic phase separation }( \pm 2) / \mathrm{h}}$ & 15 & 31 & 10 & 8 \\
\hline $\begin{array}{l}\text { Volume-based mean droplet diameter after } \\
6 \mathrm{~h}( \pm 0.10) / \mu \mathrm{m}\end{array}$ & 0.63 & 0.50 & 0.97 & 0.95 \\
\hline
\end{tabular}

Table 6.5. Characteristic times and SLS volume based mean droplet diameter measured after $6 \mathrm{~h}$ for emulsions prepared with 15, 25, 40 and $65 \mathrm{wt} \%$ toluene. 
The mechanism of destabilization may be probed by fitting the mean droplet size versus time data. The volume-based mean droplet diameter was measured every 15 minutes during the first 6 hours after emulsion fabrication. Raw data are shown in Figure 6.9 for 15, 25, 40 and 65 wt\% toluene emulsions representative of the four concentration domains. Due to the large sample volume required to perform a single SLS measurement (from $\sim 0.5$ to $\sim 3.5 \mathrm{~mL}$ depending on the oil concentration), SLS measurements using the same sample could not be performed for times longer than $6 \mathrm{~h}$.

From Figure 6.9, the volume-based mean droplet diameter of the 15, 25 and 40 wt\% oil emulsion samples, plotted against time, follow similar trends over the first 6 hour period as opposed to the 65 wt\% oil sample. In this highly concentrated sample, the plot follows the same trend as the other three concentrations from $\sim 1$ to $6 \mathrm{~h}$ with droplet growth following a different mechanism during the first hour of the emulsion destabilization. Note that for all of the emulsions droplets begin to grow within the first $~ 30$ minutes after fabrication.

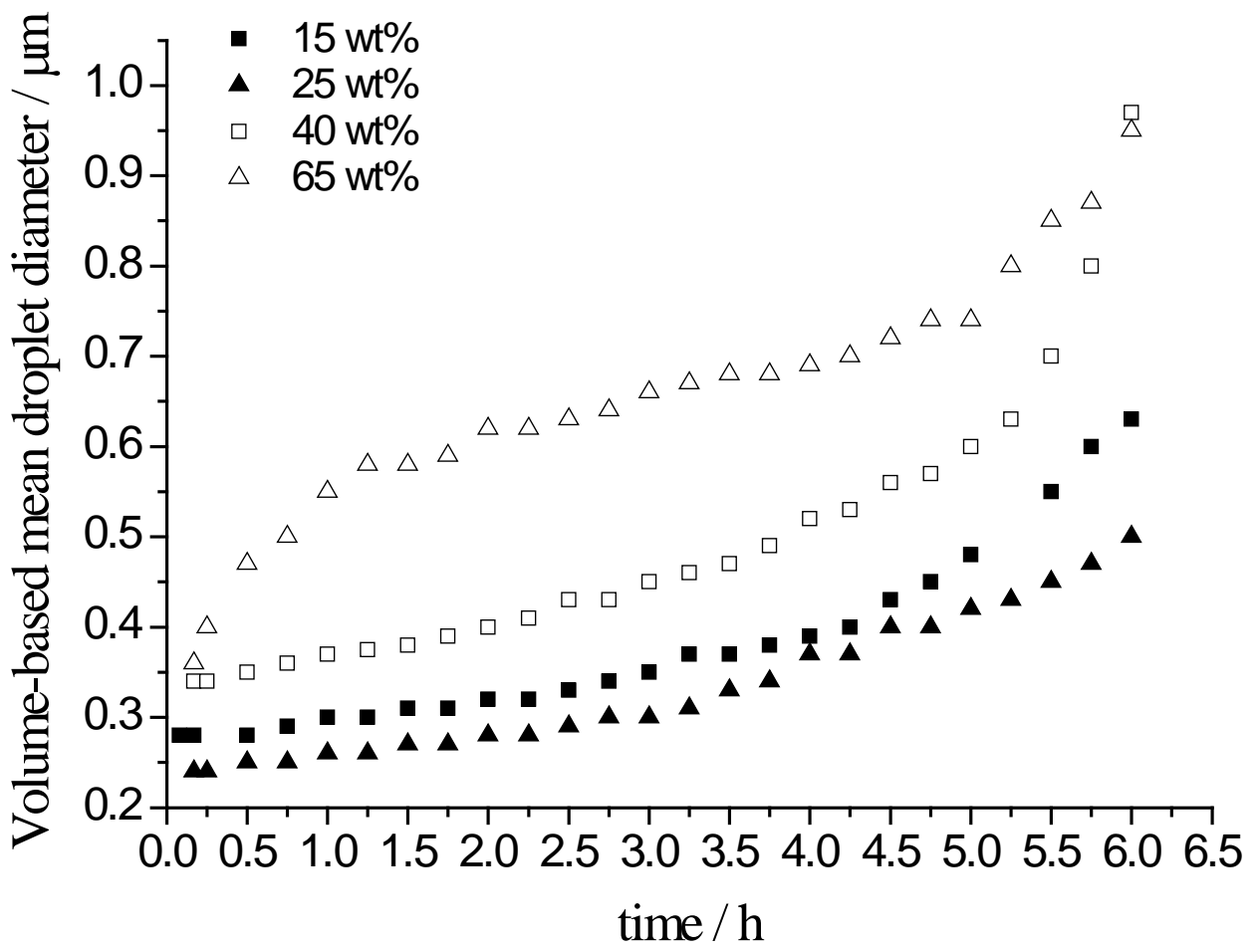

Figure 6.9. SLS volume-based mean droplet diameter plotted against time for 15, 25, 40 and $65 \mathrm{wt} \%$ toluene samples. 
Three potential mechanisms for droplet growth must be considered; (i) coalescence, (ii) standard Ostwald ripening and (iii) enhanced Ostwald ripening. When coalescence is the dominant destabilization mechanism, the plot of $1 / d^{2}$ (where $d$ is the volume-based mean droplet diameter) is linear with respect to time $[114,115]$.

On the other hand, if Ostwald ripening is the driving force for instability, the droplet growth is described by a $d^{3}$-linear dependence $[116,117]$ and for example in the presence of oil swollen micelles, which may act as oil carriers (enhanced Ostwald ripening), droplet growth follows a $d^{2}$-linear relationship against time $[84,118]$.

Applying these three fits to the raw data, the plot of $d$ versus time is best fit by a $1 / d^{2}$ linear dependence for times between $\sim 30 \mathrm{~min}$ and $6 \mathrm{~h}$ for the 15, 25 and 40 $\mathrm{wt} \%$ oil samples and between $\sim 1$ and $6 \mathrm{~h}$ for the $65 \mathrm{wt} \%$ toluene emulsions (Figure 6.10). This linear dependence coupled with the increase of the sample polydispersity over time (the size distribution becomes multimodal, Figure 6.8) indicates that coalescence is the dominant mechanism for droplet growth in the low-oil content, high-oil content and transition regions during the 6 hours following emulsification. 


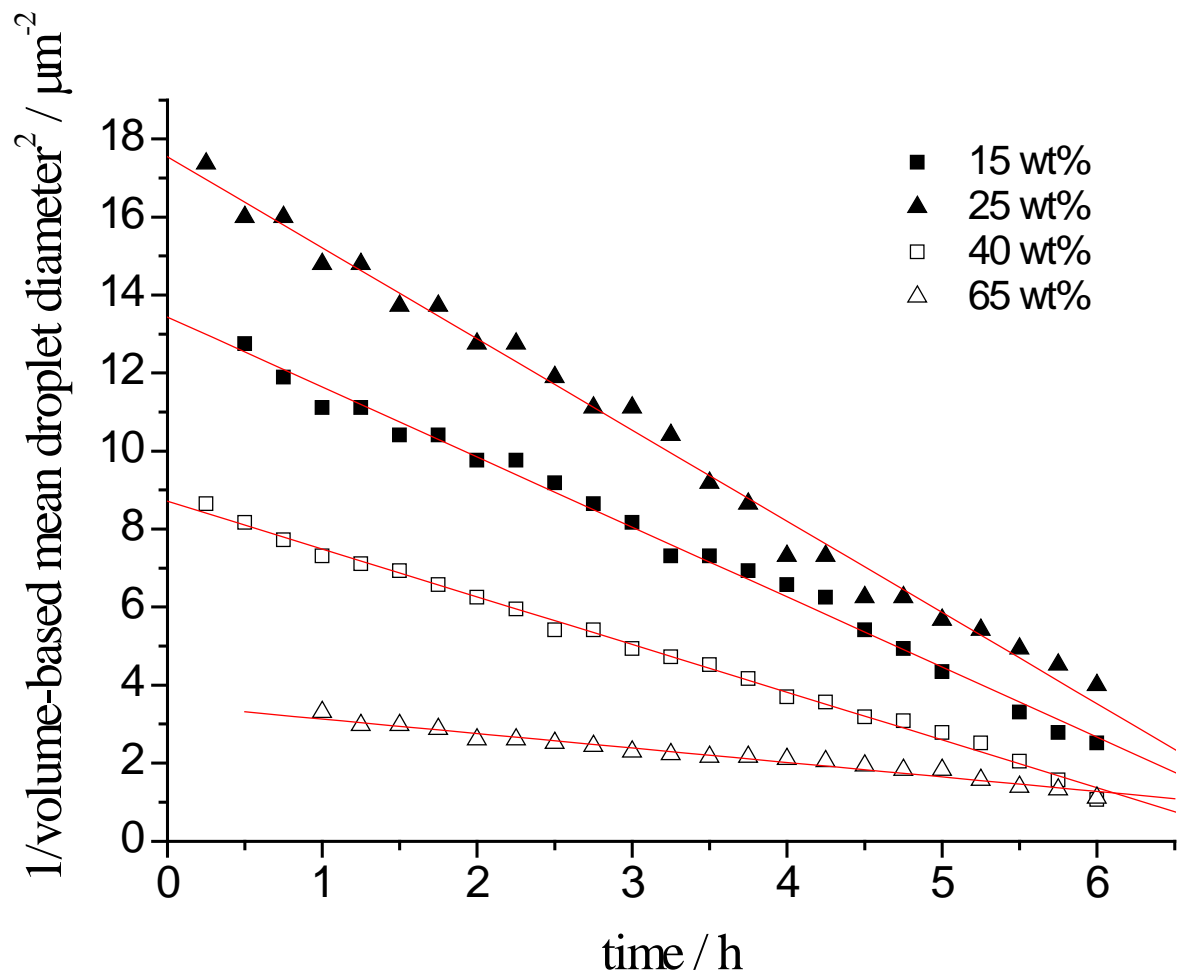

Figure 6.10. Inverse of the square volume-based mean droplet diameter plotted against time for $15,25,40$ and $65 \mathrm{wt} \%$ toluene samples.

In an emulsion destabilizing via coalescence, the mean droplet diameter varies with time according to Eq. 6.1 [115];

$$
\frac{1}{d^{2}}=\frac{1}{d_{0}^{2}}-\frac{2 \pi \omega t}{3}
$$

where $d$ is the average droplet diameter after a time $t, d_{0}$ is the average mean droplet diameter at $t=0$ and $\omega$ is the frequency of film rupture per unit of the film surface.

In Table 6.6 is summarized the frequency of film rupture per unit of the film surface or frequency of coalescence based on the linear fits shown in Figure 6.10 upon increasing toluene concentration from 15 to $65 \mathrm{wt} \%$. The rate of coalescence is expected to be an increasing function of the droplet concentration and the initial mean droplet size. By contrast, considering the 15, 40 and $65 \mathrm{wt} \%$ toluene emulsions, the frequency of coalescence is a decreasing function of oil concentration varying from $0.86 \pm 0.03$ to $0.58 \pm 0.02$ to $0.18 \pm 0.01 \mu \mathrm{m}^{-2} \mathrm{~h}^{-1}$; this despite the droplet concentration increasing for emulsions prepared with oil between 15 to $40 \mathrm{wt} \%$ (the droplet size distribution remains unchanged for fresh 
samples between 5 and $~ 55 \mathrm{wt} \%$ toluene, Figure 4.1) and the initial mean droplet size increasing by $60 \%$ from 15 and 40 to 65 wt\% oil (Figure 4.1). The frequency of coalescence for the $25 \mathrm{wt} \%$ toluene emulsions, belonging to the transition region between the low-oil content and high-oil content domains, is the largest of all the four concentrations investigated here, i.e. $\omega=1.12 \pm 0.05 \mu \mathrm{m}^{-2} \mathrm{~h}^{-1}$.

\begin{tabular}{|c|c|}
\hline Oil concentration / wt\% & $\begin{array}{c}\text { Frequency of coalescence / } \\
\mu \mathrm{m}^{-2} \mathrm{~h}^{-1}\end{array}$ \\
\hline 15 & $0.86 \pm 0.03$ \\
\hline 25 & $1.12 \pm 0.05$ \\
\hline 40 & $0.58 \pm 0.02$ \\
\hline 65 & $0.18 \pm 0.01$ \\
\hline
\end{tabular}

Table 6.6. Rate of coalescence during the first 6 hours following emulsification for emulsions prepared with 15, 25, 40 and 65 wt $\%$ toluene.

For the $65 \mathrm{wt} \%$ toluene emulsions, droplets mainly grow via coalescence for times between $\sim 1$ and $6 \mathrm{~h}$ (Figure 6.10) whereas a different destabilization mechanism describes the increase of $d$ from $\sim 10 \mathrm{~min}$ to $\sim 1 \mathrm{~h}$. Sufficient data could not be obtained within the first hour to categorically determine the destabilization mechanism; both standard and enhanced Ostwald ripening fit the data (Figure 6.11). The corresponding ripening rates are presented in Table 6.7. 


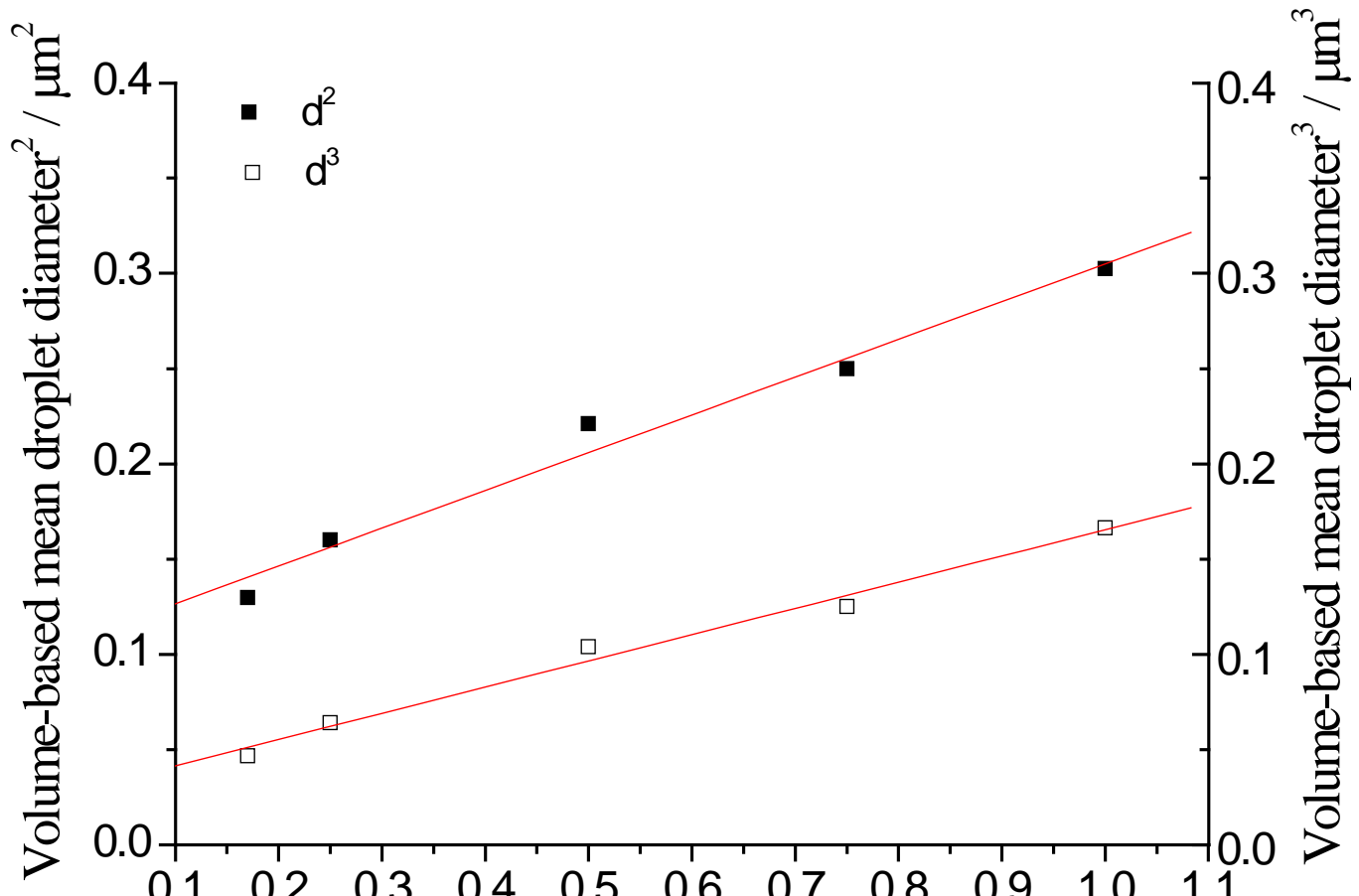

$\begin{array}{lllllllllll}0.1 & 0.2 & 0.3 & 0.4 & 0.5 & 0.6 & 0.7 & 0.8 & 0.9 & 1.0 & 1.1\end{array}$

Figure 6.11. Square and cubic volume-based mean droplet diameter plotted against time for a $65 \mathrm{wt} \%$ toluene emulsion between $10 \mathrm{~min}$ and $1 \mathrm{~h}$ post emulsification.

\begin{tabular}{|l|c|}
\hline & Ripening rate \\
\hline Enhanced Ostwald ripening $\left(\mathrm{d}^{2}\right)$ & $0.198 \pm 0.017 \mu \mathrm{m}^{2} \mathrm{~h}^{-1}$ \\
\hline Standard Ostwald ripening $\left(\mathrm{d}^{3}\right)$ & $0.138 \pm 0.009 \mu \mathrm{m}^{3} \mathrm{~h}^{-1}$ \\
\hline
\end{tabular}

Table 6.7. Ripening rates based on the linear fits for standard and enhanced Ostwald ripening mechanisms.

Droplet growth mainly occurs via coalescence during the first 6 hours following emulsion formation in all four concentration domains, with the exception that some form of Ostwald ripening takes place in the first hour following emulsification in a $65 \mathrm{wt} \%$ oil emulsion, representative of the closed-cell foam region. The fact that emulsion destabilization is driven by the same mechanism, i.e. coalescence, in both the low-oil content and high-oil content domains during the first 6 hours following emulsification suggest that the droplet growth mechanism is not related to the differences observed in the oil exchange lifetime between the two domains (Figure 5.11). This further supports the idea that the characteristics of the emulsifier thin film are different in the two domains and influence the droplet-droplet interactions. 


\subsection{PFG-NMR ageing experiments}

As toluene/Triton X-100/water emulsions age, macroscopic phase separation occurs and the continuous aqueous phase is progressively expelled from the emulsion, resulting in an increasingly concentrated upper emulsion phase. That is, upon ageing an emulsion becomes progressively depleted in both toluene molecules and oil swollen micelles as both are expelled with the water.

The decrease in the toluene molecule concentration in the continuous phase of an aged emulsion is apparent by the reduction of the fast diffusing portion of the echo attenuation of the oil signal detected in the upper concentrated emulsion only (Figure 6.12). The fast diffusing contribution corresponding to the unrestricted diffusion of toluene molecules dissolved directly in the continuous phase is significantly reduced in the upper concentrated emulsion aged for 15 days as compared with a freshly emulsified sample. This is due to the reduction of the total volume fraction of toluene dissolved in the continuous phase since by 15 days most of the aqueous phase has been expelled upon phase separation.

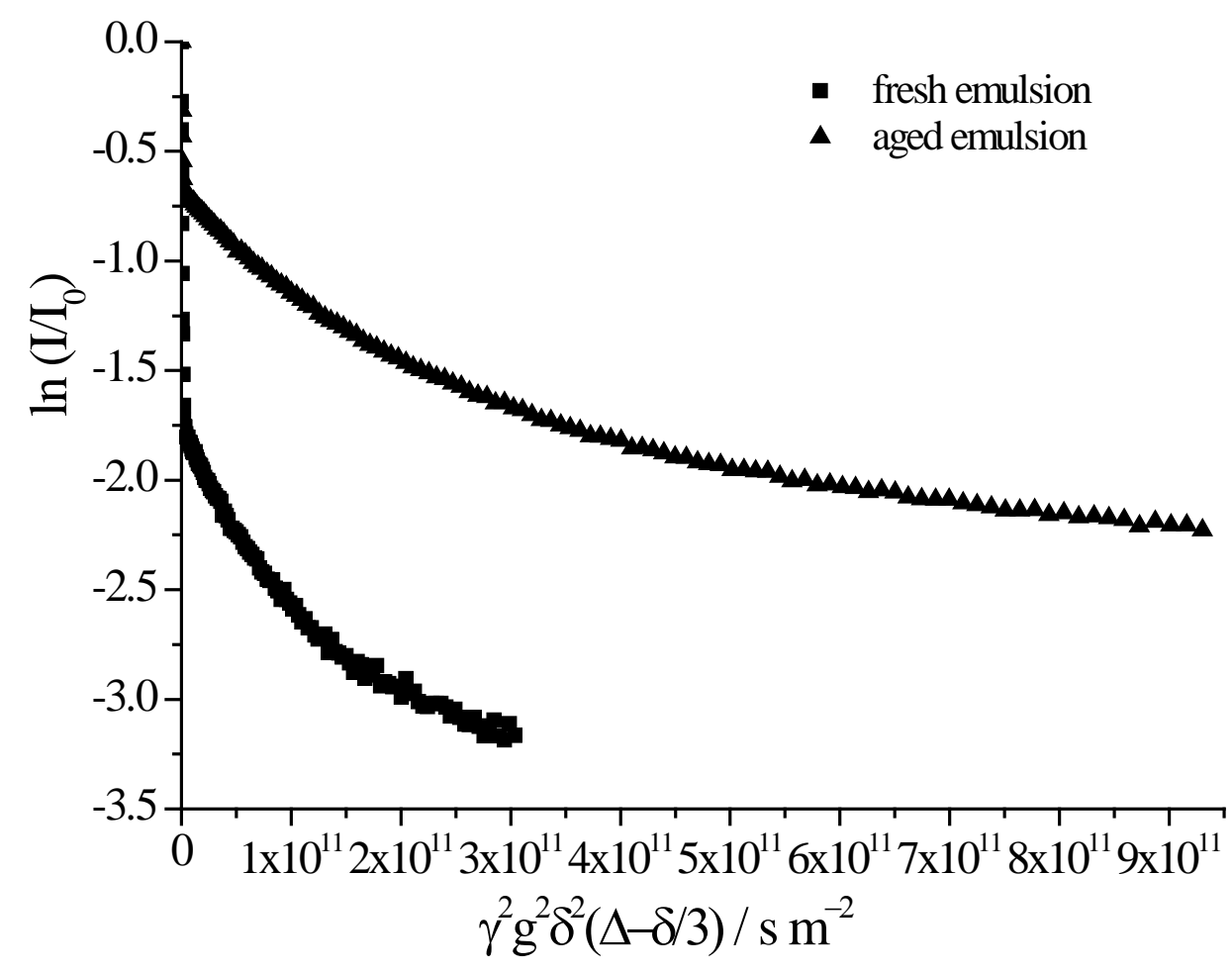

Figure 6.12. Echo attenuation of the oil signal for a $15 \mathrm{wt} \%$ toluene emulsion freshly emulsified and aged of 15 days (upper concentrated emulsion only); $\Delta=100 \mathrm{~ms}$. 
In Figure 6.13 are presented the echo attenuations of the oil signal for a fresh 40 wt\% toluene emulsion, the concentrated upper emulsion after ageing for 1 month and the corresponding expelled aqueous phase. Upon probing a phase separated $40 \mathrm{wt} \%$ toluene emulsion aged for 1 month independently of the expelled phase, it is clearly seen that in comparison with the fresh sample, the fast diffusing contribution associated with unrestricted diffusion of toluene molecules in the continuous phase has completely disappeared for all values of $\Delta$ (Figure 6.13 only shows $\Delta=100 \mathrm{~ms}$ ), as expected since most of the aqueous phase has been expelled after a month.

On the contrary, in the expelled aqueous phase of the 1 month old emulsion, the contribution of the unrestricted diffusion of toluene molecules increases compared with the fresh sample, as expected since toluene molecules now represent a larger proportion of the total volume of the sample.

The echo attenuation data follow a multiexponential decay as a function of gradient strength at $\Delta=100 \mathrm{~ms}$ for both a fresh and a 1 month old sample whereas the echo attenuation of the corresponding expelled aqueous phase is a biexponential decay for all values of $\Delta$ comprised between 6.3 and $3 \mathrm{~s}$ (Figure 6.13). This indicates that in the expelled aqueous phase the unrestricted diffusion of both toluene molecules (fast contribution) and oil swollen micelles (slow contribution) are measured. 


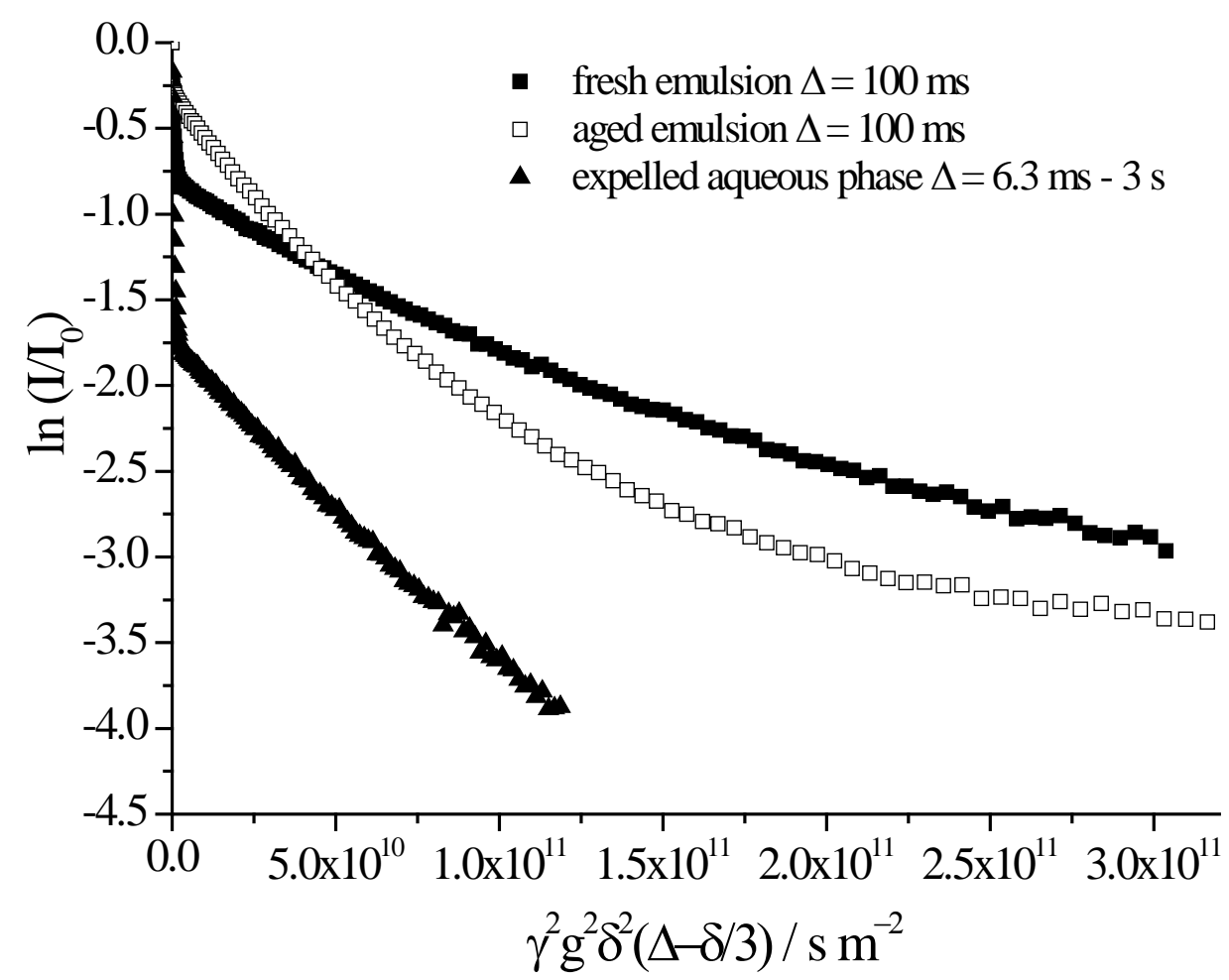

Figure 6.13. Echo attenuation of the oil signal for a freshly emulsified $40 \mathrm{wt} \%$ toluene emulsion, $\Delta=100 \mathrm{~ms}$, a $40 \mathrm{wt} \%$ toluene emulsion aged of 1 month, $\Delta=100 \mathrm{~ms}$ and the expelled aqueous phase of a $40 \mathrm{wt} \%$ toluene emulsion aged of 1 month for $\Delta$ between $6.3 \mathrm{~ms}$ and $3 \mathrm{~s}$.

The diffusion coefficients associated with the unrestricted diffusion of oil swollen micelles in the expelled aqueous phase upon macroscopic phase separation were calculated via an inverse Laplace transform for emulsions prepared with toluene between 15 and $60 \mathrm{wt} \%$ (Figure 6.14). The diffusion coefficients lie between (1.7 $\pm 0.3) \times 10^{-11}(60 \mathrm{wt} \%)$ and $(3.0 \pm 0.3) \times 10^{-11} \mathrm{~m}^{2} \mathrm{~s}^{-1}$ (30 wt\%). Using the StokesEinstein equation (Eq. 3.4), this diffusion coefficient range corresponds to a size range of 15 to $26 \mathrm{~nm}$ in diameter, which fits reasonably well with the size range of the oil swollen micelles detected by DLS (9-30 nm, Figure 4.4).

In emulsions of the low-oil content and transition regions between 15 and $35 \mathrm{wt} \%$ toluene, the diffusion coefficient remains approximately constant at an average value of $D=(2.7 \pm 0.1) \times 10^{-11} \mathrm{~m}^{2} \mathrm{~s}^{-1}$ whereas it is a decreasing function of oil concentration spanning from $(2.4 \pm 0.3) \times 10^{-11}$ to $(1.7 \pm 0.3) \times 10^{-11} \mathrm{~m}^{2} \mathrm{~s}^{-1}$ at higher oil content between 40 and $60 \mathrm{wt} \%$ toluene. This indicates that at high droplet concentration, oil swollen micelles associated with slower diffusion coefficients are on average larger in comparison with the more dilute emulsions. 


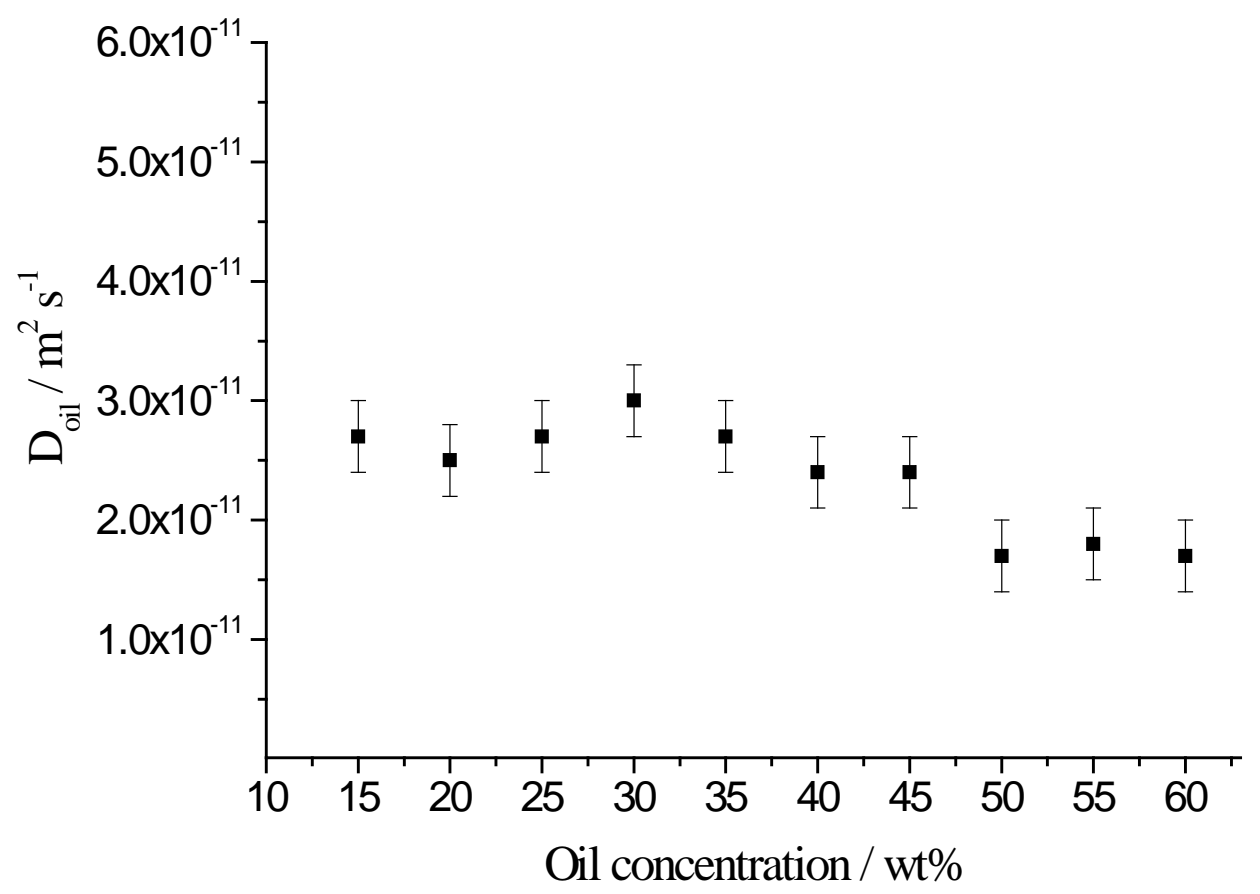

Figure 6.14. Diffusion coefficients of the oil signal measured in the expelled aqueous phase of aged emulsions prepared with toluene between 15 and $60 \mathrm{wt} \%$ for $\Delta$ between $6.3 \mathrm{~ms}$ and $3 \mathrm{~s}$.

The effect of emulsion age on the diffusion spectrum of the oil phase was evaluated by performing PGSTE experiments continuously during $70 \mathrm{~h}$ under a constant observation time, $\Delta=60 \mathrm{~ms}$.

Four emulsion samples, prepared with 15, 25, 40 and $65 \mathrm{wt} \%$ toluene, were placed in the spectrometer and left unperturbed during the ageing experiment, i.e. the coil was detecting FID signals from both the upper concentrated emulsion and the bottom expelled aqueous phase (Figure 6.15).

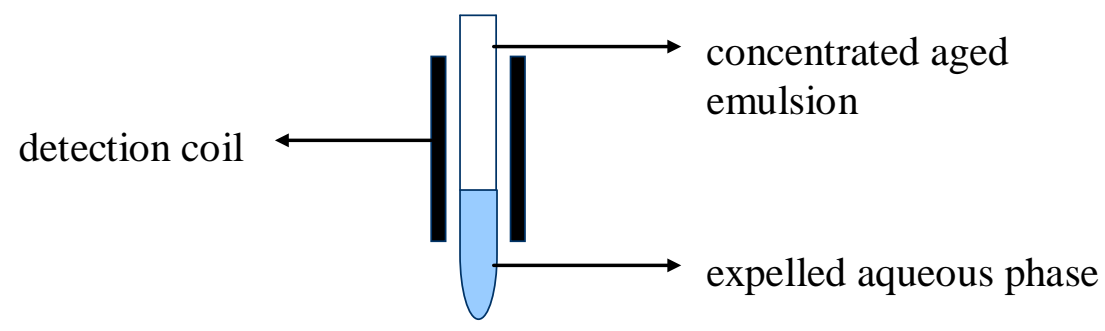

Figure 6.15. Position of a phase separated emulsion sample with respect to the spectrometer detection coil upon PFG-NMR ageing experiments.

As described in detail in chapter 5, the peaks of the diffusion spectrum may be associated with eight possible motions: unrestricted diffusion of oil droplets, 
unrestricted diffusion of oil swollen micelles, unrestricted diffusion of oil molecules within oil droplets, unrestricted diffusion of oil molecules within oil swollen micelles, restricted diffusion of oil droplets, restricted diffusion of oil swollen micelles, restricted diffusion of oil molecules within oil droplets and restricted diffusion of oil molecules within oil swollen micelles. A reminder that unrestricted diffusion of oil molecules confined within droplets and oil swollen micelles measuring $\sim 9 \mathrm{~nm}$ to $\sim 16 \mu \mathrm{m}$ diameter upon ageing cannot be detected in our experiments. However, in aged emulsions, given the increase of the average droplet size over time, restricted diffusion of oil molecules within droplets can be measured.

In Figure 6.16 are illustrated the diffusion spectra of a $15 \mathrm{wt} \%$ toluene emulsion at $\Delta=60 \mathrm{~ms}$ for $70 \mathrm{~h}$.

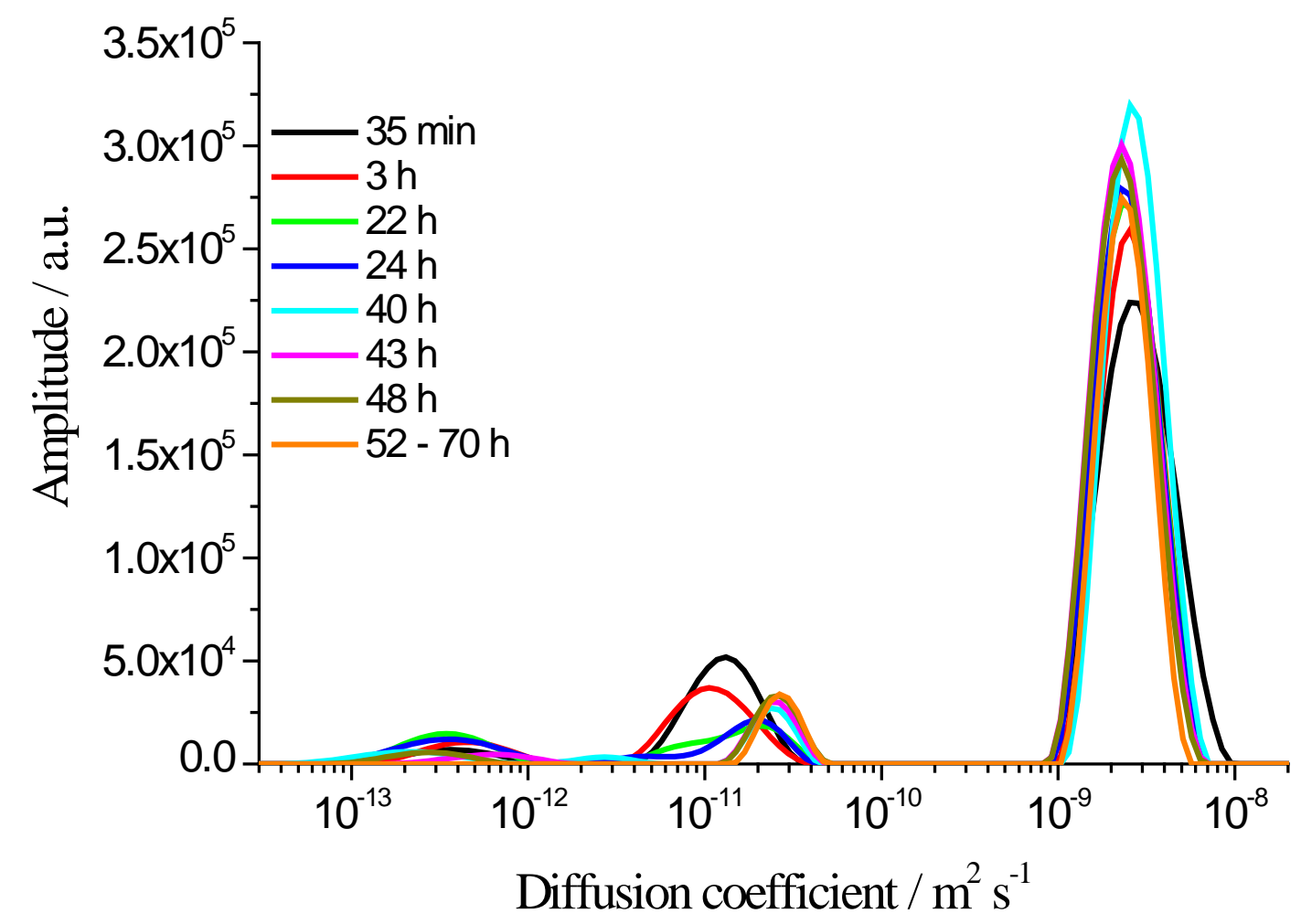

Figure 6.16. Diffusion spectra of the oil signal for a $15 \mathrm{wt} \%$ toluene emulsion over $70 \mathrm{~h}$, $\Delta=60 \mathrm{~ms}$.

The peak associated with the fastest diffusion in the spectra, centred between $~ 2.3$ and $\sim 2.6 \times 10^{-9} \mathrm{~m}^{2} \mathrm{~s}^{-1}$, pertains to the unrestricted diffusion of toluene molecules dissolved directly in the continuous phase. From 35 min to $70 \mathrm{~h}$, the amplitude of 
this peak slightly increases as the volume fraction of expelled aqueous phase in the detection volume increases upon macroscopic phase separation (Figure 6.15). Thirty five minutes following emulsification, while the emulsion is still fresh, the diffusion spectrum comprises two peaks centered at $\sim 1.3 \times 10^{-11} \mathrm{~m}^{2} \mathrm{~s}^{-1}$ (middle peak) and $\sim 3.6 \times 10^{-13} \mathrm{~m}^{2} \mathrm{~s}^{-1}$ (peak associated with slowest diffusion), respectively (Figure 6.17). For $\Delta=60 \mathrm{~ms}$, the middle peak with limits $\sim 3.0 \times$ $10^{-12}$ and $\sim 4.0 \times 10^{-11} \mathrm{~m}^{2} \mathrm{~s}^{-1}$ corresponds to the unrestricted diffusion of oil swollen micelles $(10-30 \mathrm{~nm})$ and droplets from $\sim 40 \mathrm{~nm}$ to $0.2 \mu \mathrm{m}$. The peak associated with slowest diffusion with limits $\sim 1.0 \times 10^{-13}$ and $\sim 1.2 \times 10^{-12} \mathrm{~m}^{2} \mathrm{~s}^{-1}$ refers to the restricted diffusion of droplets measuring 0.3 to $0.5 \mu \mathrm{m}$ diameter (droplets larger than $\sim 0.5 \mu \mathrm{m}$ are not detected by the instrument in fresh samples as they represent only a small volume fraction).

Upon ageing up to $22 \mathrm{~h}$, the amplitude of the peak corresponding to slowest diffusion associated with the restricted diffusion of the largest droplets increases whereas the amplitude of the middle peak decreases. This is consistent with the increase of the number of droplets measuring between $\sim 0.3$ and $3 \mu \mathrm{m}$ at the expense of those measuring between $\sim 40 \mathrm{~nm}$ and $\sim 0.2 \mu \mathrm{m}$ (Figure 6.8). Additionally, at $22 \mathrm{~h}$ the middle peak splits into two peaks centered at $\sim 7.0 \times$ $10^{-12}$ (middle left peak) and $\sim 2.0 \times 10^{-11} \mathrm{~m}^{2} \mathrm{~s}^{-1}$ (middle right peak).

From 22 to $40 \mathrm{~h}$, the middle left peak shifts to slower diffusion coefficient values and is centred at $\sim 2.7 \times 10^{-12} \mathrm{~m}^{2} \mathrm{~s}^{-1}$ by $40 \mathrm{~h}$. This indicates that the diffusion of the smallest droplets ( $40 \mathrm{~nm}-0.2 \mu \mathrm{m}$ ) also becomes restricted as the emulsion undergoes macroscopic phase separation (initiated around $15 \mathrm{~h}$ for a $15 \mathrm{wt} \%$ toluene sample).

From 22 to 48 h, upon increasing phase separation, the volume fraction of the expelled aqueous phase in the detection volume increases at the expense of that of the upper aged emulsion (Figure 6.15). As such, the amplitude of the peak associated with slowest diffusion, representing the restricted diffusion of the largest droplets, decreases. On the other hand, the middle right peak, centered around $2.0 \times 10^{-11} \mathrm{~m}^{2} \mathrm{~s}^{-1}$ at $22 \mathrm{~h}$, grows and slightly shifts to fastest diffusion coefficient values as it becomes fully separated from the middle left peak. This indicates that the middle right peak, centered at $2.7 \times 10^{-11} \mathrm{~m}^{2} \mathrm{~s}^{-1}$ after $48 \mathrm{~h}$, corresponds to pure unrestricted diffusion of the oil swollen micelles. 
At $43 \mathrm{~h}$, the middle left peak associated with the restricted diffusion of the smallest droplets $(40 \mathrm{~nm}-0.2 \mu \mathrm{m})$ continues to shift to slower diffusion coefficients and merges with the peak corresponding to slowest diffusion since the inverse Laplace transform cannot differentiate peaks that are separated by less than an order of magnitude. Therefore from $43 \mathrm{~h}$, the restricted diffusion of the droplets in the aged sample is represented by a single peak (peak associated with slowest diffusion) which shifts slightly to slower diffusion coefficients for times up to $48 \mathrm{~h}$ as the droplets are increasingly packed upon phase separation leading to a smaller displacement travelled during $60 \mathrm{~ms}$.

At $52 \mathrm{~h}$ and up to $70 \mathrm{~h}$, the coil is unable to detect a signal from the upper aged emulsion as it now represents only a small fraction of the total volume. Hence, only unrestricted diffusion of the toluene molecules and the oil swollen micelles in the expelled aqueous phase are detected.

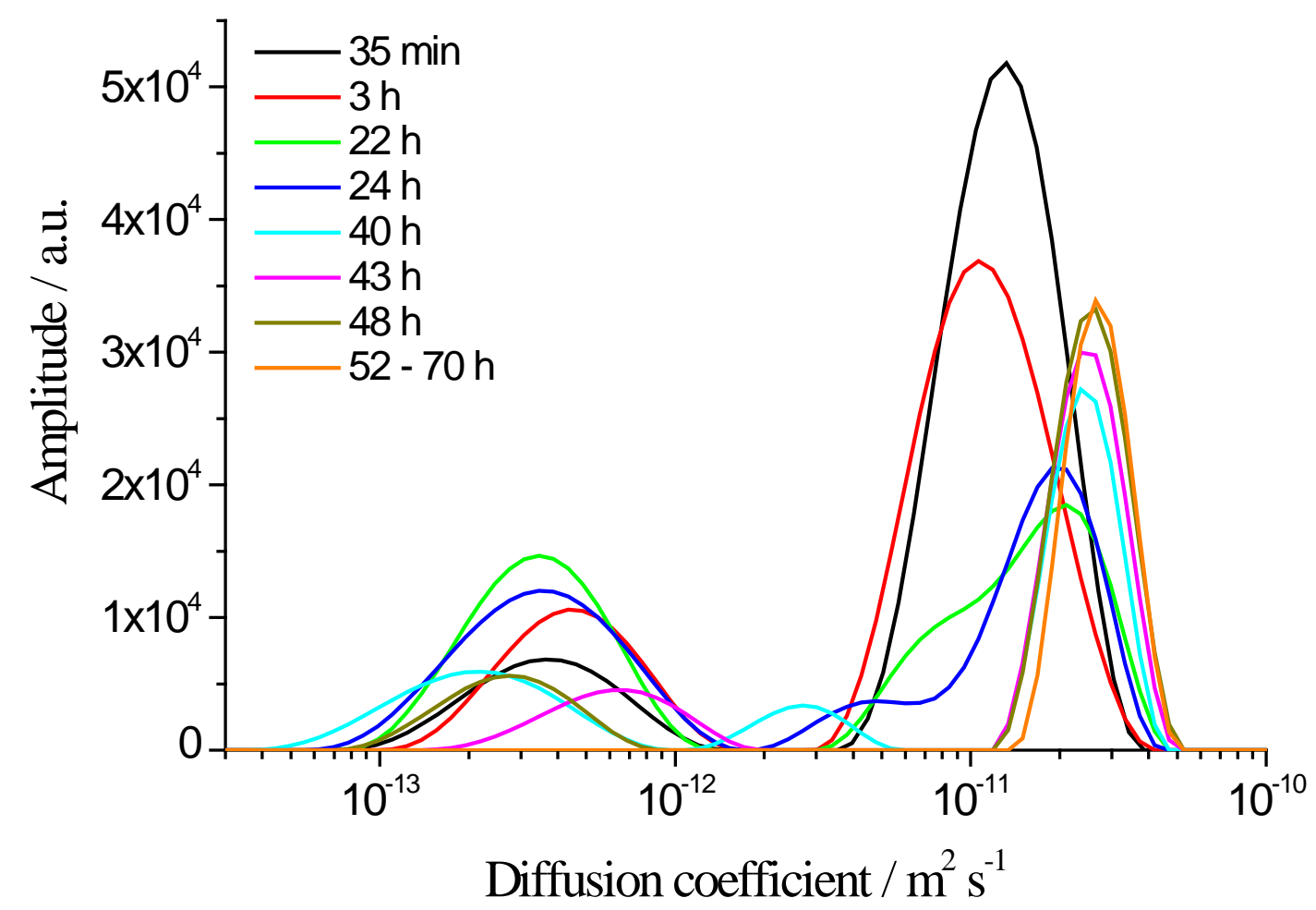

Figure 6.17. Diffusion spectra of the oil signal between $1 \times 10^{-14}$ and $1 \times 10^{-10} \mathrm{~m}^{2} \mathrm{~s}^{-1}$ for a 15 wt $\%$ toluene emulsion over $70 \mathrm{~h}, \Delta=60 \mathrm{~ms}$.

Upon increasing toluene concentration to $25 \mathrm{wt} \%$, the diffusion spectra recorded for times up to $35 \mathrm{~h}$ are similar to those of the $15 \mathrm{wt} \%$ toluene emulsion. However, since the oil volume fraction has increased, the restricted diffusion of 
oil molecules within droplets is now detected as both the peak corresponding to slowest diffusion and the middle peak shift to faster diffusion coefficient values from 35 to $70 \mathrm{~h}$ (Figure 6.18). This is evidence that restricted diffusion of oil molecules within the growing droplets is being measured since oil moves a longer distance associated with faster diffusion coefficient values during $60 \mathrm{~ms}$. Using Eq. 3.15, the peak associated with slowest diffusion mainly corresponds to the restricted diffusion of toluene molecules within droplets between 0.2 and $3 \mu \mathrm{m}$ while the middle peak corresponds to the restricted diffusion within droplets measuring 3.5 to $16 \mu \mathrm{m}$, fitting perfectly the size range measured by SLS upon ageing (Figure 6.8).

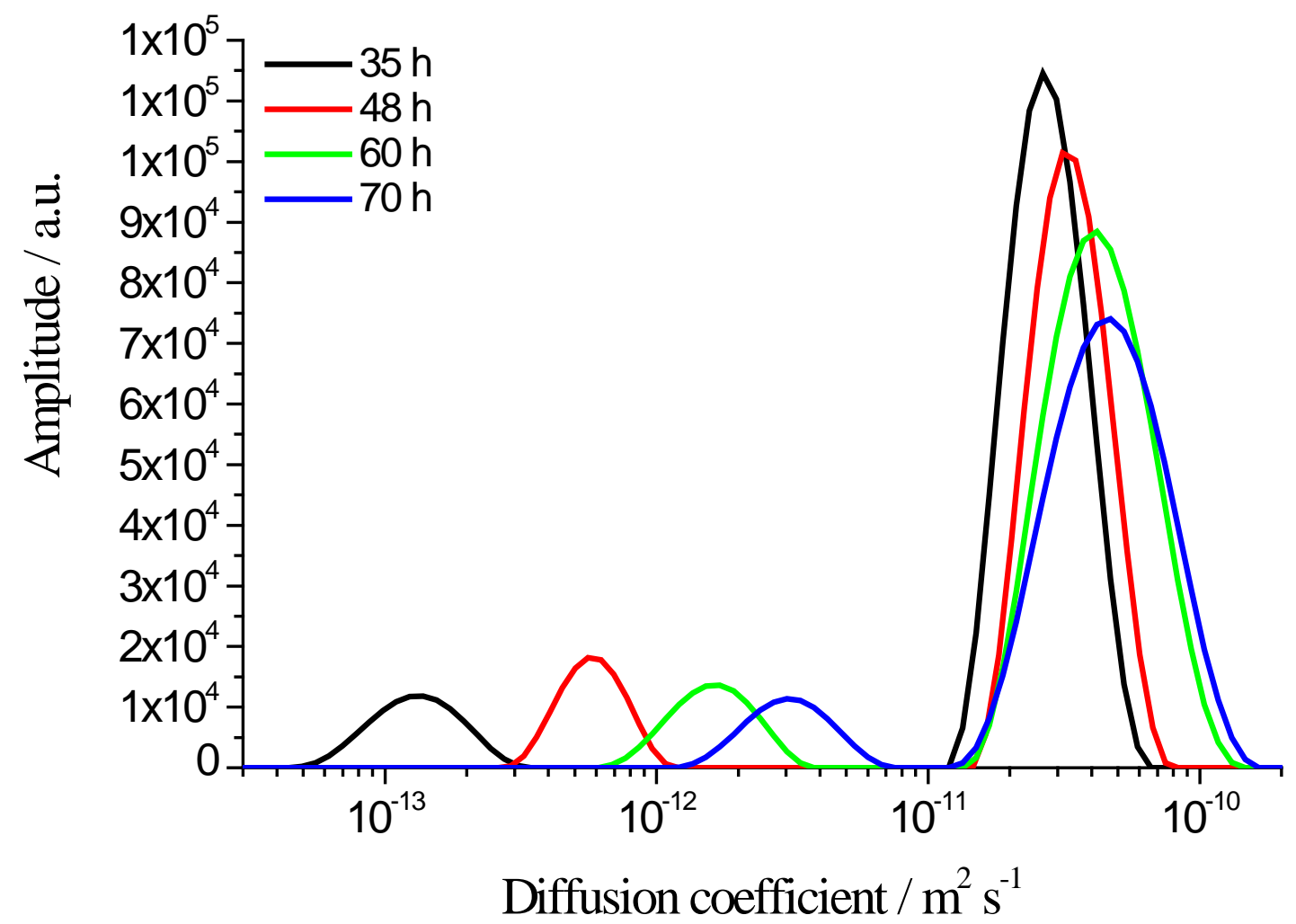

Figure 6.18. Diffusion spectra of the oil signal between $3 \times 10^{-14}$ and $2 \times 10^{-10} \mathrm{~m}^{2} \mathrm{~s}^{-1}$ for a 25 wt \% toluene emulsion between 35 and $70 \mathrm{~h}, \Delta=60 \mathrm{~ms}$.

Considering the $40 \mathrm{wt} \%$ toluene emulsions, the variation of diffusion spectra of the oil with time for $\Delta=60 \mathrm{~ms}$ are shown in Figure 6.19. 


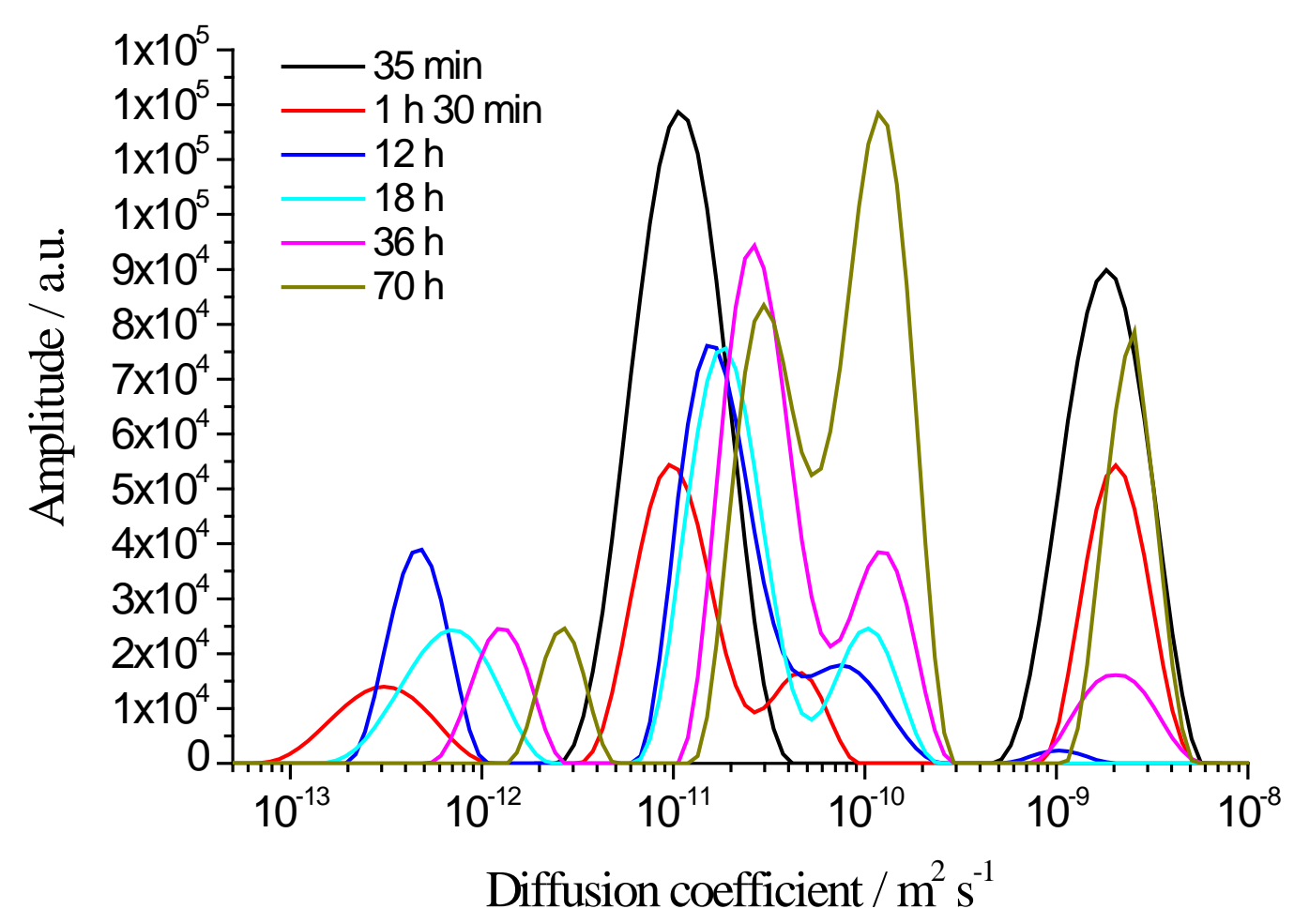

Figure 6.19. Diffusion spectrum of the oil signal for a $40 \mathrm{wt} \%$ toluene emulsion over $70 \mathrm{~h}$, $\Delta=60 \mathrm{~ms}$.

The peak associated with slowest diffusion centred around $4 \times 10^{-13} \mathrm{~m}^{2} \mathrm{~s}^{-1}$ and associated with the restricted diffusion of the largest droplets is not detected since it now lies below the detection limit of the instrument in this more concentrated sample. As such, the diffusion spectrum recorded 35 min after emulsification is comprised of only two peaks centered at $\sim 1.5 \times 10^{-11} \mathrm{~m}^{2} \mathrm{~s}^{-1}$ (middle peak) and $\sim 2.0 \times 10^{-9} \mathrm{~m}^{2} \mathrm{~s}^{-1}$ (peak corresponding to fastest diffusion). In this sample restricted diffusion of oil molecules within droplets is detected as early as $90 \mathrm{~min}$ post emulsification due to a larger oil volume fraction. This results in the appearance of a peak centred at $\sim 3.0 \times 10^{-13} \mathrm{~m}^{2} \mathrm{~s}^{-1}$ which is attributed to the restricted diffusion of oil molecules within droplets measuring 0.3 to $1 \mu \mathrm{m}$ diameter (Eq. 3.15). This peak starts to shift to faster diffusion coefficient values from $\sim 12 \mathrm{~h}$, emphasising the occurrence of droplet growth, for times up to $70 \mathrm{~h}$ (by $70 \mathrm{~h}$ this peak pertains to the restricted diffusion of oil within droplets between 1 and $2.5 \mu \mathrm{m}$ diameter).

Considering the peak associated with fastest diffusion, centered at $\sim 2.0 \times 10^{-9} \mathrm{~m}^{2}$ $\mathrm{s}^{-1}$, and associated with the unrestricted diffusion of toluene molecules dissolved directly in the continuous phase, its amplitude decreases over time and by $18 \mathrm{~h}$ it 
has totally disappeared. This indicates that after macroscopic phase separation is initiated ( $10 \mathrm{~h}$ for a $40 \mathrm{wt} \%$ toluene emulsion), the volume fraction of expelled phase is negligible and cannot be detected by the coil. However, after $36 \mathrm{~h}$ the volume of expelled phase has increased sufficiently, resulting in the reappearance of this peak, centered at $\sim 2.0 \times 10^{-9} \mathrm{~m}^{2} \mathrm{~s}^{-1}$.

With the peaks associated with fastest and slowest diffusions accounted for, the remaining peaks need to be considered. In addition to the peak centered at $\sim 1.0 \times$ $10^{-11} \mathrm{~m}^{2} \mathrm{~s}^{-1}$ corresponding to the unrestricted diffusion of oil swollen micelles and the restricted diffusion of droplets up to $0.2 \mu \mathrm{m}$ at $\mathrm{t}=1 \mathrm{~h} 30 \mathrm{~min}$, a fourth peak with limits of $\sim 3.0 \times 10^{-11}$ and $\sim 1.0 \times 10^{-10} \mathrm{~m}^{2} \mathrm{~s}^{-1}$ is detected. The increase of the amplitude of the peak centered at $\sim 4.5 \times 10^{-11} \mathrm{~m}^{2} \mathrm{~s}^{-1}$ over time coupled with its shift to faster diffusion coefficients is suggestive of restricted diffusion of oil molecules within the largest droplets (8 to $19 \mu \mathrm{m}$ diameter, Eq. 3.15). This is in agreement with the SLS data (Figure 6.8).

On the other hand, the shift of the peak initially centered at $\sim 1.0 \times 10^{-11} \mathrm{~m}^{2} \mathrm{~s}^{-1}$ to faster diffusion coefficients may be attributed to two contributions including the increase of the signal from the expelled bottom phase at the expense of the signal from the upper emulsion and the detection of the restricted diffusion of oil molecules within medium size droplets (3 to $7 \mu \mathrm{m}$, Eq. 3.15).

Finally, the diffusion spectra of the highly concentrated $65 \mathrm{wt} \%$ toluene emulsion upon ageing ( $\Delta=60 \mathrm{~ms}$ ) are shown in Figure 6.20. 


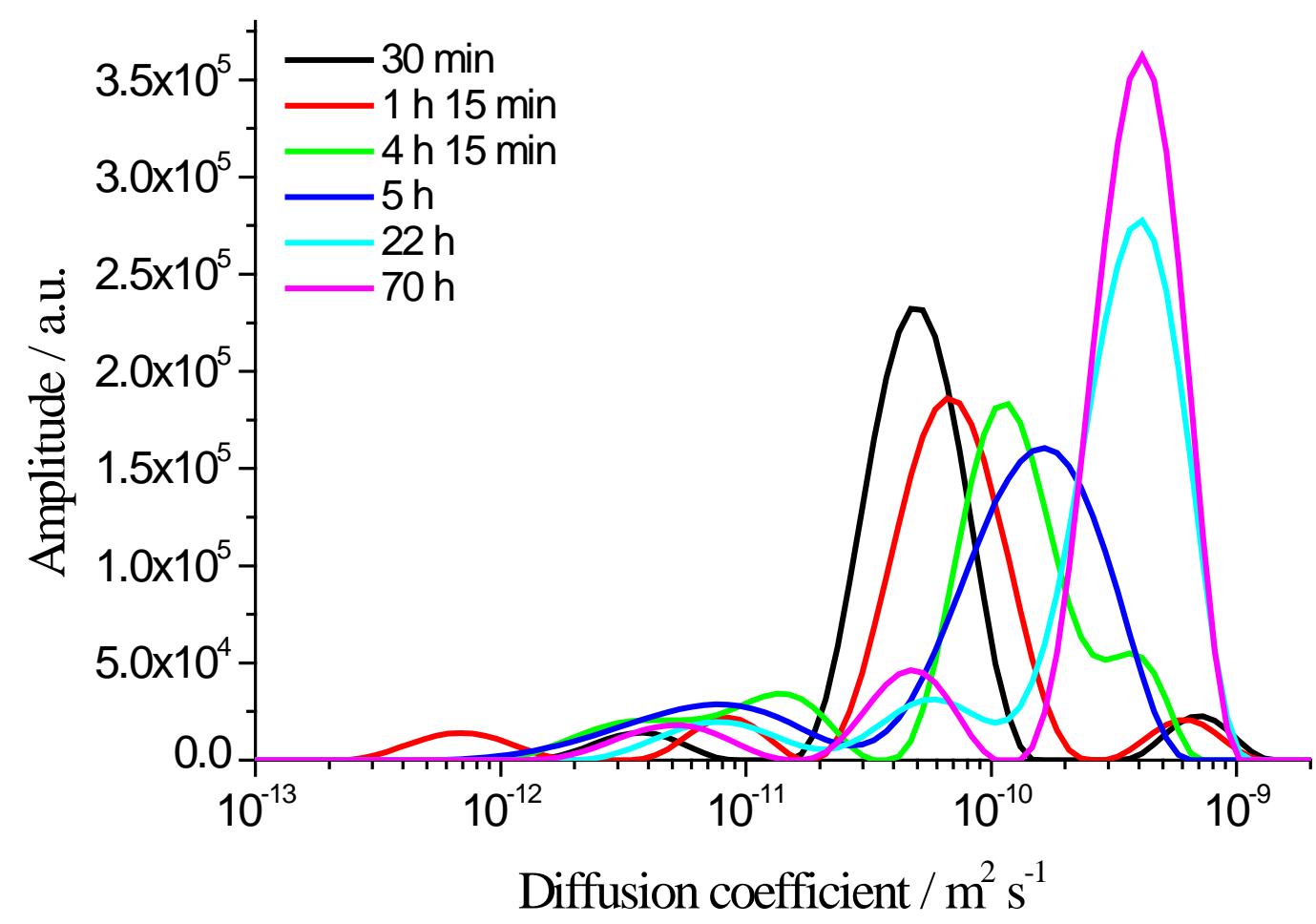

Figure 6.20. Diffusion spectra of the oil signal for a $65 \mathrm{wt} \%$ toluene emulsion over $70 \mathrm{~h}$, $\Delta=60 \mathrm{~ms}$.

Thirty five minutes following emulsification, three peaks centered at $\sim 3.8 \times 10^{-12}$, $\sim 4.7 \times 10^{-11}$ and $\sim 7.3 \times 10^{-10} \mathrm{~m}^{2} \mathrm{~s}^{-1}$ are present in the distribution. The associated motions have been described in chapter 5. Briefly, the peak centered at $\sim 3.8 \times$ $10^{-12} \mathrm{~m}^{2} \mathrm{~s}^{-1}$ is the combination of the unrestricted diffusion of the smallest droplets (40 $\mathrm{nm}$ to $\sim 0.2 \mu \mathrm{m}$ ) and the restricted diffusion of the oil within the medium size droplets ( $\sim 1$ to $\sim 3 \mu$ m diameter). The most intense peak centered at $\sim 4.7 \times 10^{-11} \mathrm{~m}^{2} \mathrm{~s}^{-1}$ is the superposition of the unrestricted diffusion of the oil swollen micelles and a part of the unrestricted diffusion of toluene molecules dissolved in the continuous phase. The peak associated with fastest diffusion, centered at $\sim 7.3 \times 10^{-10} \mathrm{~m}^{2} \mathrm{~s}^{-1}$, which is not always apparent in the spectrum at $\Delta$ $=60 \mathrm{~ms}$, corresponds to the unrestricted diffusion of toluene molecules dissolved in the continuous phase.

Upon ageing, the contribution of the diffusion within the largest droplets (the restricted diffusion of the droplets larger than $\sim 0.3 \mu \mathrm{m}$ diameter lies below the detection limit of the spectrometer) in the diffusion spectra is significant compared with that of the diffusion of the smallest droplets, as expected, since the amplitude of the NMR signal is proportional to volume. Note that the restricted 
diffusion of oil within droplets between 0.5 and $1.5 \mu \mathrm{m}$ diameter (fourth peak centered at $\sim 7.0 \times 10^{-13} \mathrm{~m}^{2} \mathrm{~s}^{-1}$ ) is detected only 75 min following emulsification for two reasons. The first is that the droplets involved in this motion may not represent a sufficiently large volume fraction to be detected and the second is that the corresponding diffusion peak is located too close to the detection limit of the instrument at $35 \mathrm{~min}$ (slower diffusion coefficients).

By $5 \mathrm{~h}$, the peaks initially centered at $\sim 7.0 \times 10^{-13}$ and $\sim 8.5 \times 10^{-12} \mathrm{~m}^{2} \mathrm{~s}^{-1}(\mathrm{t}=1 \mathrm{~h}$ $15 \mathrm{~min}$ ) have merged to a single peak centered at $\sim 7.5 \times 10^{-12} \mathrm{~m}^{2} \mathrm{~s}^{-1}$ attributed to the restricted diffusion of toluene molecules within droplets measuring $\sim 1$ to $\sim 5$ $\mu \mathrm{m}$ diameter.

As far as the most intense peak centered at $\sim 4.7 \times 10^{-11} \mathrm{~m}^{2} \mathrm{~s}^{-1}$ at $\mathrm{t}=35 \mathrm{~min}$ is concerned, it moves to faster diffusion coefficients between $35 \mathrm{~min}$ and $5 \mathrm{~h}$ mainly because it progressively incorporates the peak corresponding to the fastest diffusion coefficients (pure unrestricted diffusion of the oil molecules dissolved in the continuous phase). However, upon ageing between 5 and $70 \mathrm{~h}$, the restricted diffusion of the oil within the largest droplets up to $\sim 16 \mu \mathrm{m}$ becomes a significant contribution of this peak, resulting in an increase of amplitude. Because of the shift of the most intense peak to faster diffusion coefficients, a peak centered at $\sim 6.0 \times 10^{-11} \mathrm{~m}^{2} \mathrm{~s}^{-1}$ and associated with the unrestricted diffusion of the oil swollen micelles as well as the restricted diffusion of the oil within droplets between 5 to $11 \mu \mathrm{m}$ becomes separated from the most intense peak by $\sim 22 \mathrm{~h}$. The most intense peak overweights the presence of very large droplets in the system, i.e. between 12 and $16 \mu \mathrm{m}$ (see SLS data, Figure 6.8).

From the above analysis with an observation time, $\Delta=60 \mathrm{~ms}$, the NMR diffusion spectra fit well the SLS droplet size distribution measured upon ageing for the four concentration domains.

Emulsion age significantly affects the time scale at which oil exchange between droplets occurs in the system, i.e. the NMR switching time, $\Delta_{\text {switch, }}$ between a multiexponential and a single exponential decay varies as a function of time (Figure 6.21). 


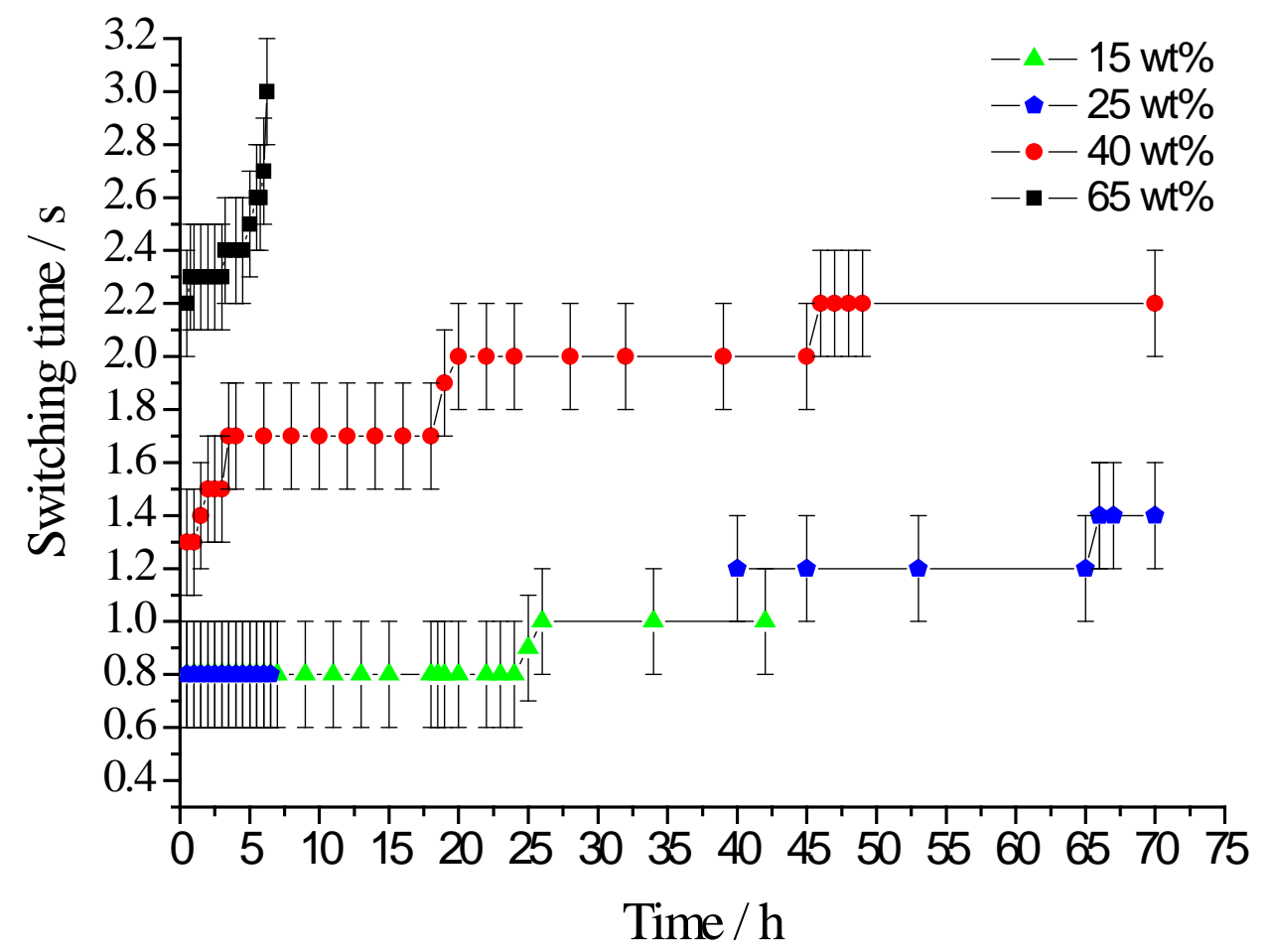

Figure 6.21. NMR switching time between a multiexponential and a single exponential decay as a function of emulsion's age for toluene concentration of 15, 25, 40 and $65 \mathrm{wt} \%$.

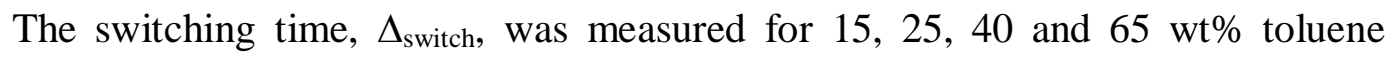
emulsions that were left unperturbed in the spectrometer for $70 \mathrm{~h}$ (Figure 6.15 and Figure 6.21). This switching time was determined as the time for which the echo attenuation of the oil signal changes from a multiexponential to a single exponential decay as a function of gradient strength upon increasing the observation time, $\Delta$, as seen in Figure 5.1.

Note that the measurement of $\Delta_{\text {switch }}$ was not possible over the entire $70 \mathrm{~h}$ period for both the 15 and $25 \mathrm{wt} \%$ toluene emulsions due to the appearance of a droplet size gradient (Figure 6.6). In the $15 \mathrm{wt} \%$ oil sample, the spectrometer coil detects a signal mainly from the bottom emulsion layer, i.e. the smallest droplets of the system (Figure 6.7), and the expelled aqueous phase between $\sim 40$ and $\sim 70$ h. As such, during that period the measured value of $\Delta_{\text {switch }}$ is erroneous since the detection volume of the coil does not include the whole volume of emulsion. The same is true for a $25 \mathrm{wt} \%$ oil sample between $\sim 7$ and $\sim 35 \mathrm{~h}$.

Despite the "gap” in the NMR data shown in Figure 6.21 for both the 15 and 25 wt\% oil samples, for the four oil concentrations investigated, $\Delta_{\text {switch }}$ is an increasing function of emulsion age. 
For the highest toluene concentration investigated, i.e. $65 \mathrm{wt} \%, \Delta_{\text {switch }}$ increases from $\sim 2.2 \mathrm{~s}$ around $30 \mathrm{~min}$ following emulsification to $\sim 3.0 \mathrm{~s}$ after $\sim 6.25 \mathrm{~h}$ (Figure 6.21). For times longer than $\sim 6.75 \mathrm{~h}, \Delta_{\text {switch }}$ is longer than the longest observation time available ( $3.0 \mathrm{~s})$ therefore it cannot be determined and the echo attenuation follows a multiexponential decay as a function of gradient strength for all values of $\Delta$.

Upon decreasing oil concentration to $40 \mathrm{wt} \%$ toluene, $\Delta_{\text {switch }}$ changes from $\sim 1.3 \mathrm{~s}$ $(\mathrm{t}=\sim 30 \mathrm{~min})$ to $\sim 2.2 \mathrm{~s}(\mathrm{t}=\sim 46$ to $70 \mathrm{~h})$. For a $25 \mathrm{wt} \%$ oil emulsion, $\Delta_{\text {switch }}$ increases from $\sim 0.8(\mathrm{t}=\sim 30 \mathrm{~min})$ to $\sim 1.4 \mathrm{~s}(\mathrm{t}=\sim 66$ to $70 \mathrm{~h})$. Finally, in a $15 \mathrm{wt} \%$ oil, $\Delta_{\text {switch }}$ changes from $\sim 0.8$ to $\sim 1 \mathrm{~s}$ over a $26 \mathrm{~h}$ period $\left(\Delta_{\text {switch }}\right.$ could not be determined for times longer than $\sim 42 \mathrm{~h}$ due to the occurrence of a droplet size gradient).

The motional rate of a droplet is proportional to the droplet size as seen by the Stokes-Einstein equation (Eq. 3.4). As the emulsion mean droplet size increases upon ageing, droplets move and interact with each other, on average, more slowly as compared with the fresh emulsions, resulting in the decrease of the droplet encounter frequency, hence the decrease of the rate of oil exchange via permeation and/or reversible coalescence. This is consistent with $\Delta_{\text {switch }}$ being an increasing function of the emulsion age as seen here (Figure 6.21).

Moreover, it is known from the measurement of both the motional averaging of the oil diffusion and the value of $\Delta_{\text {switch }}$ upon increasing the droplet collision frequency from 5 to $\sim 55 \mathrm{wt} \%$ toluene using PFG-NMR (Figure 5.7 and Figure 5.11), that the rigidity of the interfacial region, determined by the surfactant packing at the oil/water interface, significantly hinders the oil transfer through steric repulsive forces.

In Figure 6.22 is plotted $\Delta_{\text {switch }}$ versus the SLS volume-based mean droplet diameter for a $65 \mathrm{wt} \%$ toluene emulsion. The observation time, $\Delta_{\text {switch }}$, is linearly dependent on the emulsion mean droplet size within the first 6 hours following emulsion formation, i.e. before macroscopic phase separation begins from $\sim 8 \mathrm{~h}$. This is consistent with the time scale of the oil exchange being inversely proportional to the droplet collision frequency while maintaining a constant surfactant packing within the first 6 hours following emulsification. 


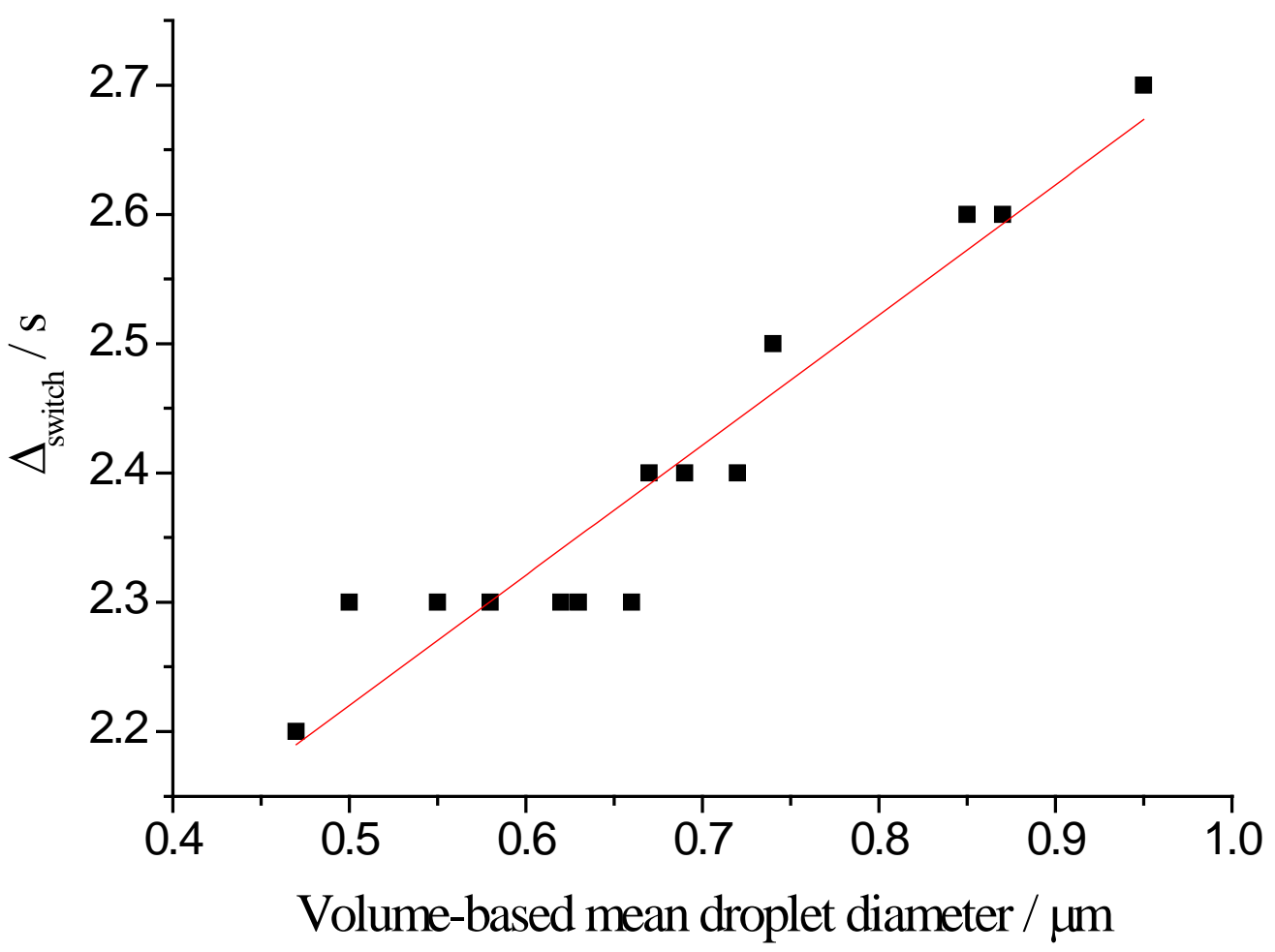

Figure 6.22. NMR switching time versus SLS mean droplet diameter within the first 6 hours following emulsification for an emulsion prepared with 65 wt \% toluene.

For the $40 \mathrm{wt} \%$ toluene emulsions, the $\Delta_{\text {switch }}$ versus SLS volume-based mean droplet size plot is consistent with a linear relationship only within the first $\sim 4 \mathrm{~h}$ following emulsification (Figure 6.23). This suggests that the increase of the mean droplet size superposed to the concomitant decrease of the droplet collision frequency is no longer the sole contribution to the variation of the time scale of the oil exchange process once macroscopic phase separation has been initiated (from $\sim 10 \mathrm{~h}$ ). The decrease of the mean interdroplet spacing upon phase separation, as the aqueous continuous phase is continuously expelled, facilitates the oil exchange process and leads to the increase of the rate of exchange as seen by the slowdown of the $\Delta_{\text {switch }}$ function over time from $\sim 4$ to $\sim 70$ h (Figure 6.24). 


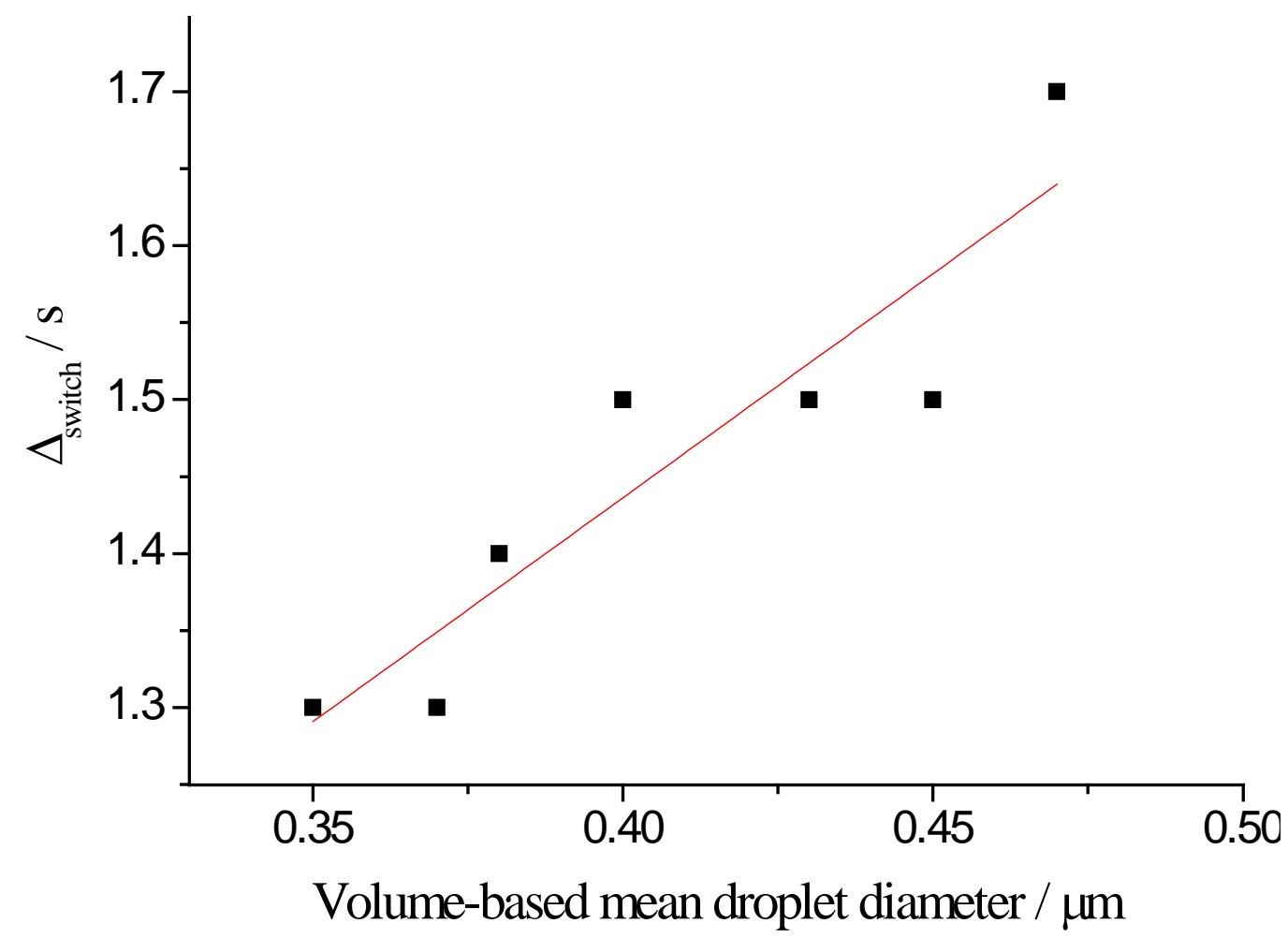

Figure 6.23. NMR switching time versus SLS mean droplet diameter within the first 4 hours following emulsification for an emulsion prepared with $40 \mathrm{wt} \%$ toluene.

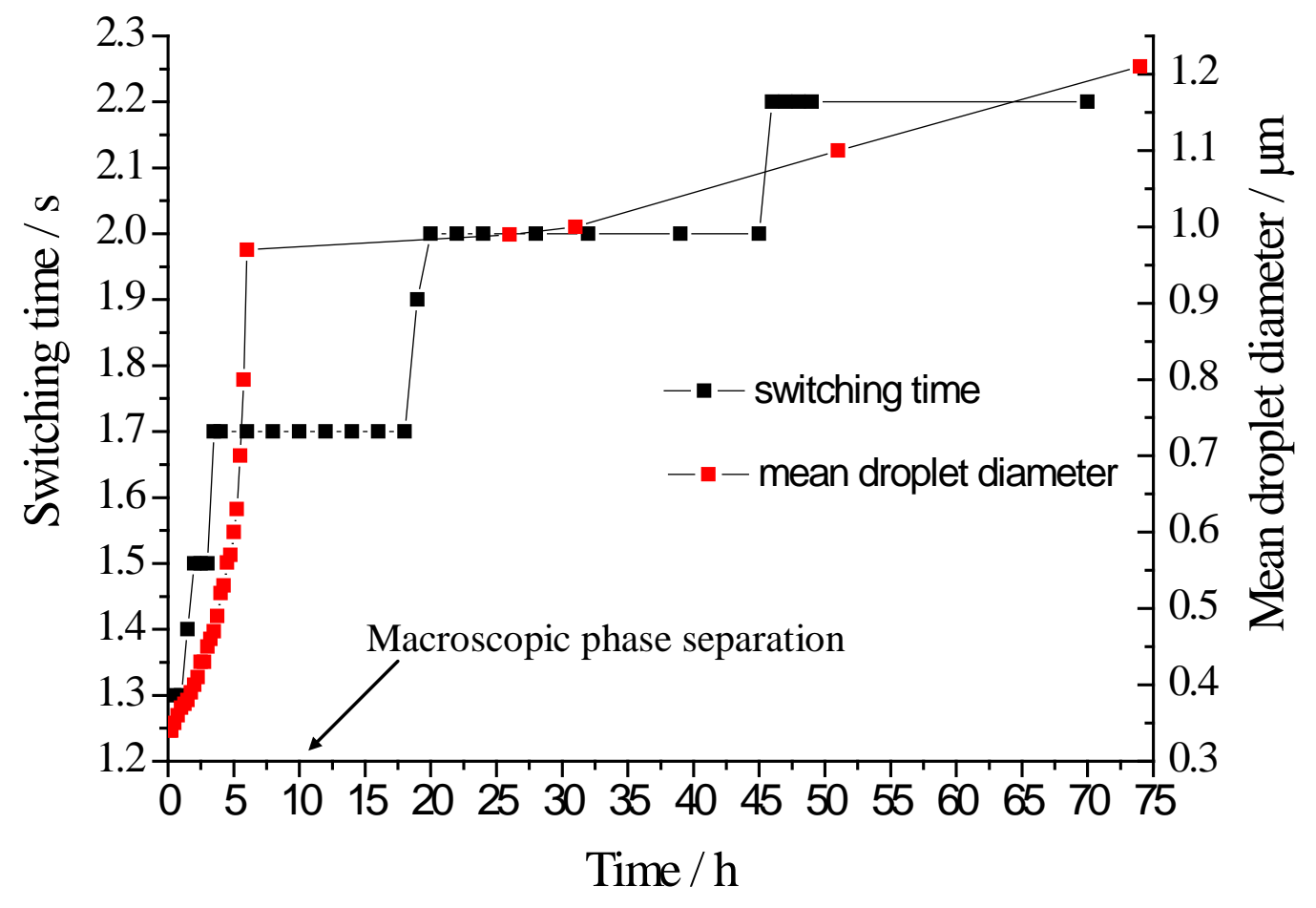

Figure 6.24. NMR switching time and SLS mean droplet diameter versus time for an emulsion prepared with $40 \mathrm{wt} \%$ toluene. 
For the more dilute samples, the switching time remains unchanged within the first few hours of the emulsion life, e.g. $\Delta_{\text {switch }}=0.8 \mathrm{~s}$ within the first 24 hours following emulsion formation for a 15 wt\% toluene sample, despite droplet growth occurring (Figure 6.25) and macroscopic phase separation being initiated (note that due to the presence of a droplet size gradient, a complete investigation of the dependence of the switching time on the droplet size was not possible). These data suggest that the increase of the emulsion mean droplet size and the concomitant decrease of the droplet encounter frequency upon ageing has a limited impact on the rate of oil exchange at low oil content. This corroborates the very low droplet coverage, facilitating oil exchange, in both the low-oil content and transition regions as compared with the high-oil content and closed-cell foam domains.

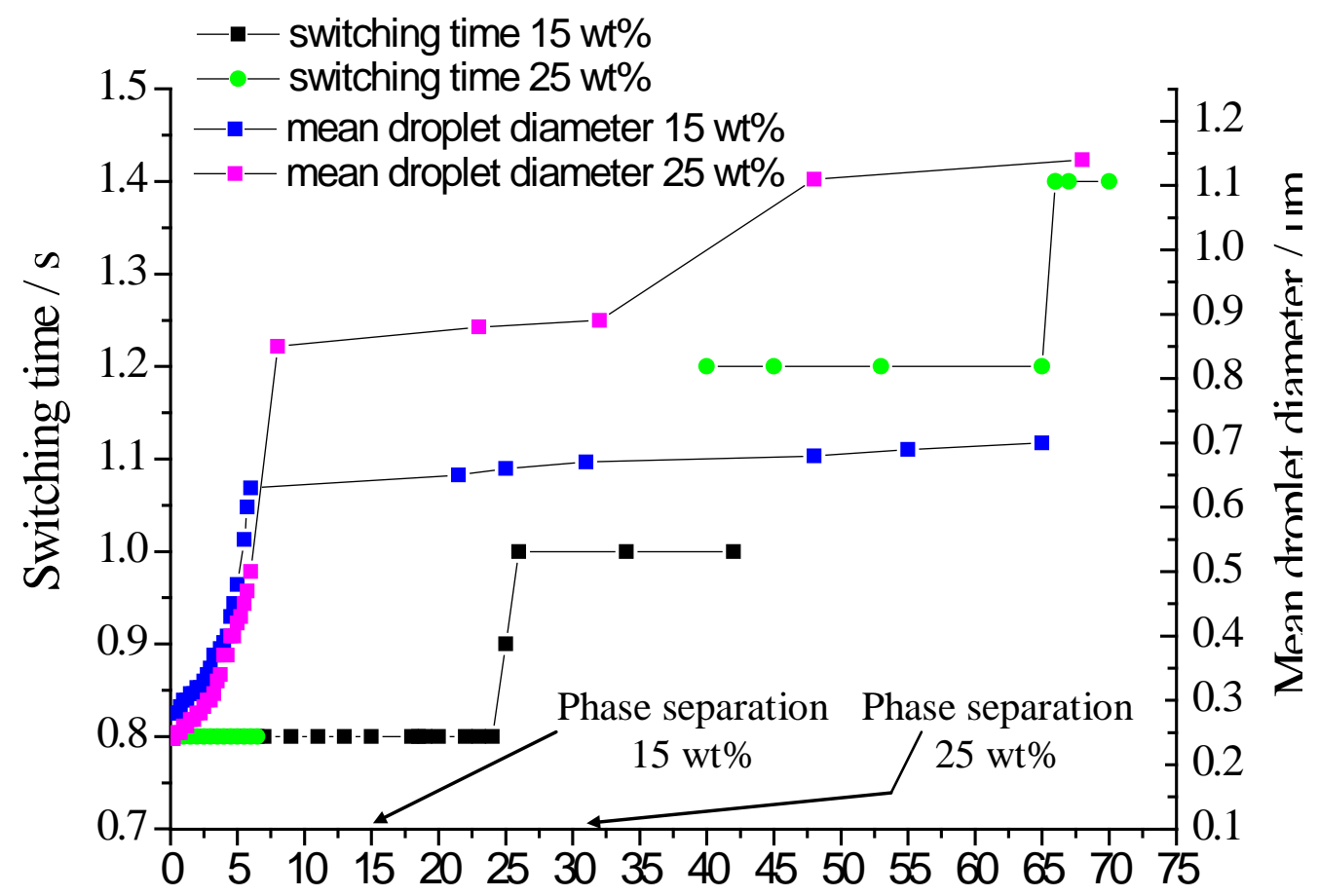

Figure 6.25. NMR switching time and SLS mean droplet diameter versus time for a 15 and 25 wt\% oil emulsions.

In similarity with the switching time, the motional averaging of the oil diffusion, calculated by an inverse Laplace transform based on the single exponential decay raw data, varied with emulsion age (Figure 6.26). At very high concentration, 65 $\mathrm{wt} \%$ toluene, the motional averaging of the oil rises from $\sim 5.0 \times 10^{-11}(\sim 30 \mathrm{~min})$ to $\sim 6.3 \times 10^{-11} \mathrm{~m}^{2} \mathrm{~s}^{-1}$ ( $3 \mathrm{~h} 15 \mathrm{~min}$ to $6 \mathrm{~h}$ ) and from $\sim 1.5 \times 10^{-11}(\sim 30 \mathrm{~min})$ to $\sim 4.7$ 
$\times 10^{-11} \mathrm{~m}^{2} \mathrm{~s}^{-1}$ ( 50 to $\left.70 \mathrm{~h}\right)$ for a $40 \mathrm{wt} \%$ toluene emulsion. For the $25 \mathrm{wt} \%$ toluene emulsions, the motional averaging increases from $\sim 1.5 \times 10^{-11}$ ( 30 min) to $\sim 3.7 \times 10^{-11} \mathrm{~m}^{2} \mathrm{~s}^{-1}(\sim 65$ to $70 \mathrm{~h})$. Finally at lower oil content, $15 \mathrm{wt} \%$ toluene, the motional averaging initially at $\sim 1.3 \times 10^{-11} \mathrm{~m}^{2} \mathrm{~s}^{-1}$ rises to $\sim 1.9 \times 10^{-11} \mathrm{~m}^{2} \mathrm{~s}^{-1}$ ( $\sim 14$ to 42 h). For all four toluene concentrations investigated, the motional averaging of the oil diffusion increases with emulsion age which is consistent with the mean droplet size increasing with emulsion age leading to oil molecules being able to travel a longer distance within the droplets over the same duration before exchanging.

Furthermore, the motional averaging of the oil diffusion increases with oil concentration in aged emulsions (Figure 6.26) as opposed to being independent of oil concentration as seen in fresh samples between 5 and $55 \mathrm{wt} \%$ oil (Figure 5.7). This suggests that the balance between the increase of the droplet concentration, facilitating oil exchange, and the enhancement of the surfactant packing, hindering oil exchange, changes with ageing. For a given concentration, the decrease of the mean interdroplet spacing upon macroscopic phase separation leads to the further increase of the droplet collision frequency. This results in the kinetic stabilisation of the system via the enhancement of the surfactant packing at the oil/water interface similarly to that observed upon increasing oil content in fresh emulsions from 5 to $\sim 55$ wt \% toluene. The variation of the droplet coverage with ageing may proceed via the reservoir of surfactant molecules within oil swollen micelles. The reduction of the number of oil swollen micelles upon phase separation, as these are progressively expelled from the emulsion phase, may account for a weaker enhancement of the surfactant packing at the interface. 


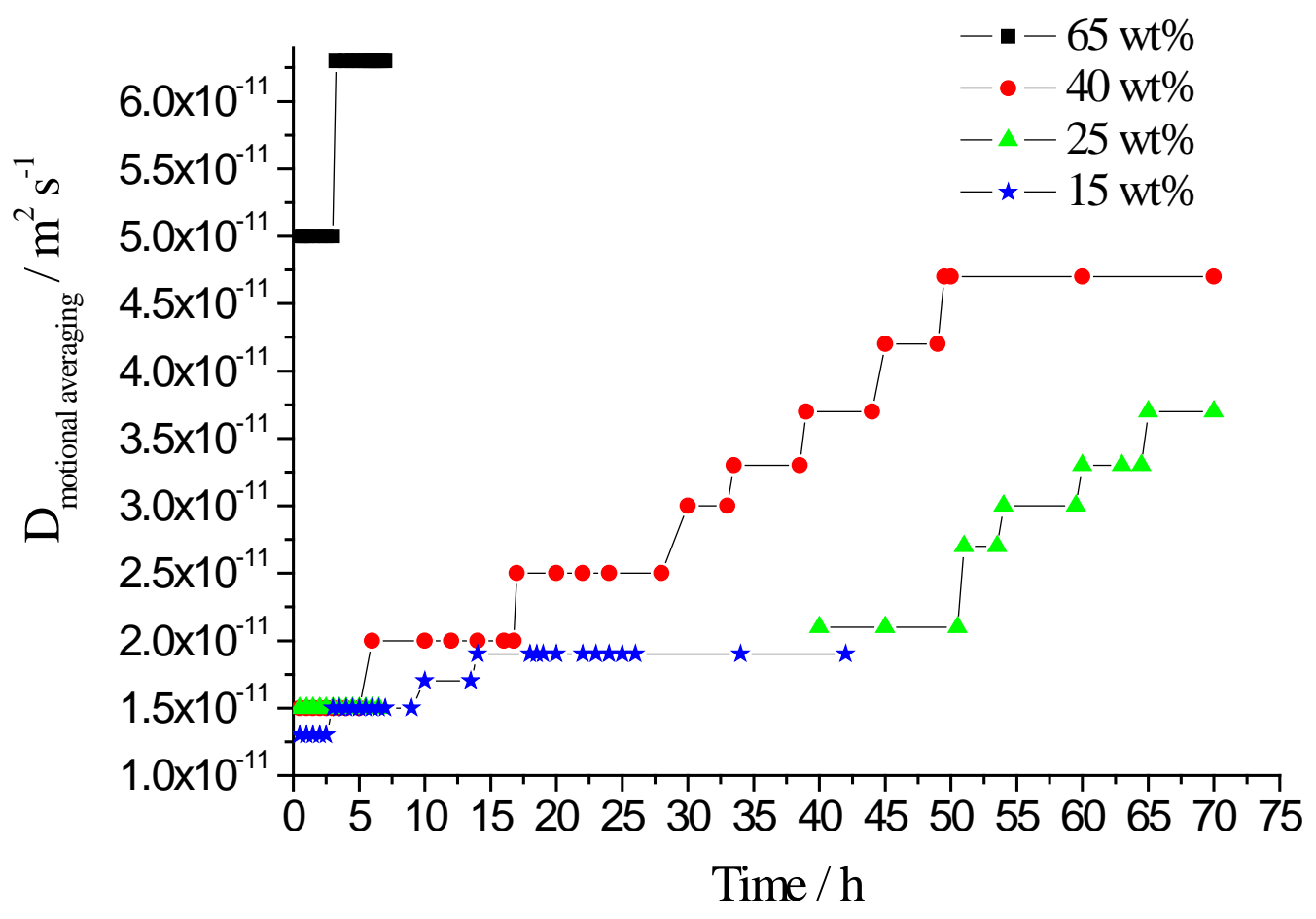

Figure 6.26. Motional averaging of the oil diffusion plotted against time, calculated by an inverse Laplace transform for emulsions prepared with 15, 25, 40 and $65 \mathrm{wt} \%$ toluene, $\Delta \geq$ $\Delta_{\text {switch. }}$

\subsection{Discussion}

Ageing experiments allow us to probe how the microstructure of toluene/Triton $\mathrm{X}-100 /$ water emulsions is adjusted by energy input to optimize the stability of the oil droplets upon decreasing the mean interdroplet distance. At low oil content between 5 and $20 \mathrm{wt} \%$, the contribution of the droplet collision frequency in controlling the rate of macroscopic phase separation predominates over the droplet coverage in the early stages of emulsion destabilization as seen by the time at which macroscopic phase separation is initiated and the time of the rapid increase of the amount of expelled aqueous phase being both decreasing functions of oil concentration between 5 and $20 \mathrm{wt} \%$.

Upon decreasing the mean interdroplet distance, i.e. in the more concentrated emulsions between 40 and $70 \mathrm{wt} \%$ toluene or in the final stage of phase separation at lower oil content once most of the aqueous phase has been expelled, the contribution of the interface properties (surfactant packing) in controlling the rate of phase separation is enhanced as confirmed by the macroscopic phase separation data. The time to reach complete phase separation is an increasing 
function of oil concentration between 5 and $20 \mathrm{wt} \%$ toluene and at high oil content from 40 to $65 \mathrm{wt} \%$ toluene the time at which phase separation is initiated is constant and the times to reach complete phase separation are on average longer than those of the more diluted samples.

The SLS time data indicate that toluene/Triton X-100/water emulsions mainly destabilize via a coalescence mechanism independently of oil concentration although the contribution of Ostwald ripening is significant within the first hour of the emulsion life for the highly concentrated samples (65 wt\% oil). These data are consistent with the trends observed in the literature. As a polar and a moderately water-soluble oil, toluene is expected to generate $\mathrm{O} / \mathrm{W}$ emulsions that are unstable with regard to coalescence as opposed to water-soluble oils of lower polarity which are more stable with respect to coalescence but more unstable with regard to Ostwald ripening [119]. Additionally, Georgieva et al. [120] found similar unstability mechanisms in highly concentrated $\mathrm{O} / \mathrm{W}$ emulsions. In the first stages of destabilization, Ostwald ripening is the predominant mechanism because of the small droplet size and the high Laplace pressure. Once a critical droplet size is reached, coalescence becomes the main destabilization mechanism.

The quantitative analysis of the SLS experiments with regard to toluene/Triton X100/water emulsions indicates that the frequency of coalescence is a decreasing function of toluene concentration within the first $6 \mathrm{~h}$ following emulsification (Table 6.6). In a $15 \mathrm{wt} \%$ toluene sample, droplets coalesce at a rate of $0.86 \pm 0.03$ $\mu \mathrm{m}^{-2} \mathrm{~h}^{-1}$ as compared with $0.58 \pm 0.02 \mu \mathrm{m}^{-2} \mathrm{~h}^{-1}$ for a $40 \mathrm{wt} \%$ toluene emulsion despite droplets being closer to each other and colliding more frequently. At very high oil content between 60 and $70 \mathrm{wt} \%$, droplets are on average $60 \%$ larger than in the more dilute samples (Figure 4.1). This should lead to an increase of the rate of coalescence because larger droplets coalesce faster with others than do small ones due to a larger surface contact area with their neighbours [121]. However, the rate of coalescence measured for a $65 \mathrm{wt} \%$ toluene sample is $0.18 \pm 0.01 \mu \mathrm{m}^{-2}$ $\mathrm{h}^{-1}$. Therefore, these data corroborate that the enhancement of the surfactant packing at the interface upon increasing toluene content occurs as suggested by the concomitant increase of the time scale of the oil exchange measured by PFGNMR ( $\Delta_{\text {switch }}$ versus toluene concentration, Figure 5.11) as well as the macroscopic phase separation data. Upon increasing oil concentration, a higher 
degree of surfactant packing increases the stability of the droplets by providing a more robust steric repulsive barrier and decreasing the interfacial tension between toluene and water [113].

It is widely accepted that the HLB number introduced by Griffin [5] cannot be used as the only indicator to predict the spontaneous mean curvature of a surfactant layer. In addition to the chemical structure, the geometry and the size of the molecules of the surfactant film, those of the dispersed phase and the spontaneous mean curvature of the film can be affected, for example, by the temperature [122, 123], the addition of a background electrolyte or the composition of the system [124, 125] particularly through mutual interactions between oil, surfactant and water molecules and the hydration of the surfactant head groups [126]. The balance between the dipole-dipole repulsive forces of the surfactant headgroups, the steric repulsive forces between the surfactant tails and the hydrophobic attractive forces between the surfactant tails defines the most energetically favourable surfactant packing and the film equilibrium mean curvature. Israelachvili et al. [127] introduced the concept of critical packing parameter $(C P P)$ to describe the curvature of a surfactant layer at equilibrium in empty micelles, oil swollen micelles or microemulsion droplets. CPP is defined as

$$
C P P=\frac{v}{a_{0} l_{c}}
$$

where $v$ is the volume of the surfactant tail chain, $a_{0}$ is the equilibrium area per surfactant head at the interface and $l_{c}$ is the length of the fully extended tail.

The CPP has been widely used as a simple tool to characterize the self-assembly of surfactants [128, 129]. This parameter is not simply dependant of the geometrical shape and size of the surfactant molecules [104]. Indeed, the parameters $v$ and $l_{c}$ depend on the strength of the steric repulsive forces between the surfactant tails and between the surfactant tails and the oil molecules confined within the droplets. The equilibrium area per surfactant head group $a_{0}$ is controlled by the balance between the hydrophilic repulsive forces between the headgroups and the attractive forces between the hydrophobic chains. Hence, the 
CPP, inversely proportional to the HLB value, gives an insight of the spontaneous mean curvature of the surfactant layer.

The PFG-NMR data associated with the unrestricted motion of the oil swollen micelles in toluene/Triton X-100/water emulsions indicate that the oil swollen micelles get larger upon increasing toluene content. As oil swollen micelles are thermodynamically stable, the mean curvature of the Triton X-100 layer in the oil swollen micelles is the equilibrium curvature. Therefore, the equilibrium curvature of the Triton X-100 layer becomes less positive upon increasing toluene concentration. It is more energetically favorable for the Triton X-100 layer to curve less towards the oil upon increasing toluene content due to the dehydration of the ethylene oxide head groups upon decreasing the water concentration, resulting in the decrease of the distance between the head groups while the distance between the tails is maintained [104]. A less positive equilibrium curvature of the Triton X-100 layer should lead to oil exchange (via oil permeation or reversible coalescence) and destabilization via coalescence being facilitated since the rate of hole nucleation is proportional to the energy barrier for the formation of a local negative curvature [78]. However, instead of converting to a W/O emulsion described by a negative mean curvature, the $\mathrm{O} / \mathrm{W}$ system is kinetically stabilized at high oil content by the enhancement of the surfactant packing at the interface in order to counter the augmentation of the droplet encounter frequency. The augmentation of the steric repulsive forces imposed by a higher surfactant packing predominates over the decrease of the energy barrier for hole nucleation, hence the observed decrease of the rate of coalescence and oil exchange upon increasing oil concentration.

Moreover, the increase of the size of the oil swollen micelles upon increasing oil content, as measured by PFG-NMR, also corroborates the increase of the surfactant packing at the droplet surface. Assuming the volume of oil solubilised within the micelles remains unchanged, the decrease of the surface area of the micelles upon increasing toluene concentration confirms that more surfactant is available to be placed at the oil/water interface.

Oil swollen micelles can be formed in the continuous phase of emulsions if there is an excess of surfactant molecules once the surface of the droplets is covered. The driving force for the formation of oil swollen micelles is the tendency of the surfactant layer to adopt its equilibrium mean curvature [84]. As such, oil swollen 
micelles act as a surfactant reservoir to adjust the amount of surfactant required at the interface over time. As the mean interdroplet spacing decreases upon macroscopic phase separation, oil droplets are stabilized by steric repulsion due to the enhancement of the surfactant packing. However, the oil swollen micelles are progressively expelled from the emulsion phase upon phase separation and so does the reservoir of surfactant molecules. This results in the motional averaging of the oil diffusion being an increasing function of oil concentration upon ageing. There are several examples in the literature of the manipulation of the surfactant packing at the surface of emulsion droplets upon changing the composition of the system. For example, Oehlke et al. [130] investigated corn oil-in-water emulsions stabilized by two different ionic emulsifiers, sodium dodecyl sulfate (SDS) and cetyl trimethyl ammonium bromide (CTAB). The concentration of emulsifier within the interfacial region and in the continuous phase under the form of micelles and monomers were measured using small angle neutron scattering, ultrafiltration and dialysis. The surfactant headgroup area $a_{s}$ was calculated based on the concentration of surfactants at the interface coupled to the total interfacial area measured by DLS. They found that $a_{s}$ continues to decrease upon increasing the total CTAB concentration in the system even after oil swollen micelles solubilised in the continuous phase have been formed. On the contrary with SDS, once oil swollen micelles have been formed, maximum coverage of the interface is achieved resulting in the surfactant head group area $a_{s}$ being independent of the total surfactant concentration introduced in the system.

The solubilisation of cosurfactants, such as long chain alcohols, in the oil phase of $\mathrm{O} / \mathrm{W}$ emulsions before emulsification can lead to the transfer of additional surfactant molecules from the oil swollen micelles to the interfacial region. For example in the study of James-Smith et al. [131] the addition of dodecanol in hexadecane-in-water emulsions imposes a $23 \%$ increase in the amount of SDS available for the droplet surface as measured by ultrafiltration since the total interfacial area is enhanced and the mean droplet size decreases. This is due to the shielding of the electrostatic repulsion between SDS headgroups, the maximization of the van der Waals attractive interactions between the hydrophobic tails and the ion-dipole interactions among the polar groups $\mathrm{OH}$ and $\mathrm{SO}_{4}$ providing a greater stability of the interfacial film by increasing the surfactant packing at the interface. 
In the toluene/Triton X-100/water emulsions of the transition region between $\sim 25$ and 35 wt $\%$ toluene, these are associated with the fastest rates of coalescence (1.12 $\pm 0.05 \mu \mathrm{m}^{-2} \mathrm{~h}^{-1}$ for a $25 \mathrm{wt} \%$ toluene sample, Table 6.6), hence the smallest surfactant packing at the interface highlighting the significant instability of these samples. This is consistent with the previous study of Liu et al. [40], in which a bicontinuous toluene/Triton X-100/water emulsion with a zero mean curvature could be stabilized at low energy input with $30 \mathrm{wt} \%$ toluene indicating that the spontaneous mean curvature of Triton X-100 progressively becomes less positive upon increasing toluene concentration from 5 to $30 \mathrm{wt} \%$. The surfactant layer within this transition region of $\sim 25$ to $\sim 35 \mathrm{wt} \%$ toluene is very unstable since it does not preferentially curve towards the oil or towards the water. In this region the curvature strongly depends on the bending elasticity of the monolayer [132]. Finally, the emulsion mean droplet size is an important determinant of the rate of oil exchange as seen by $\Delta_{\text {switch }}$ being an increasing function of droplet size. At high oil content, the time scale of the oil exchange is proportional to the mean droplet size or inversely proportional to the droplet collision frequency for a given concentration whereas at low oil content, the surfactant packing is so weak that the rate of oil exchange is unaffected by an increase in the mean droplet size.

\subsection{Conclusion}

Comparing toluene/Triton X-100/water emulsions of the low-oil content region (5 to $\sim 20 \mathrm{wt} \%$ oil) to those of the high-oil content ( 40 to $\sim 55 \mathrm{wt} \%$ oil) and closedcell foam ( $\sim 60$ to $70 \mathrm{wt} \%$ oil) domains, the packing of Triton X-100 molecules at the surface of oil droplets is enhanced as evidenced by the macroscopic phase separation data and the rate of coalescence upon increasing toluene concentration. The observed increase of the surfactant packing upon increasing oil content results from the kinetic stabilization of the system against the augmentation of the droplet collision frequency coupled to the reduction of the energy cost for hole nucleation. The decrease of the energy barrier to oil exchange originates from the spontaneous mean curvature of the Triton X-100 layer becoming less positive upon dehydration of the surfactant head groups as evidenced by the oil swollen micelles getting larger with oil concentration. 
The rate of oil transfer between droplets decreases upon the ageing of emulsions due to droplets becoming larger, hence diffusing more slowly, as seen by the

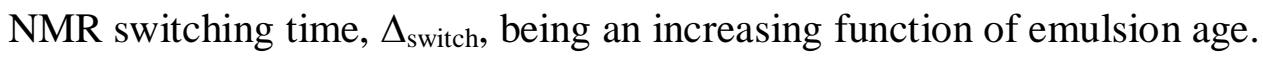




\section{Chapter 7 Variation of the nature of the oil in oil/Triton $\mathrm{X}-100 /$ water emulsions}

\subsection{Introduction}

Changing the nature of the oil from toluene to $p$-xylene to octane in oil/Triton $\mathrm{X}$ 100/water emulsions allows the specific interaction between Triton X-100 and the oil to be probed and the stability of the surfactant thin film to be manipulated. From toluene to $p$-xylene, the addition of one methyl group to the oil molecule affects the properties of the interfacial domain resulting in the modification of the microstructure and the stability of these emulsions. The characteristics of the interface are controlled by the oil molecular size, the degree of penetration of the oil molecules into the interfacial thin film and the mutual chemical affinities between the oil, the surfactant and the water molecules $[3,104]$.

In this chapter is described how the droplet size distribution and the macroscopic stabilities of $p$-xylene emulsions are affected by the variation of oil concentration from 2.5 to 70 wt\% in comparison with the toluene system.

Moreover, the occurrence of oil exchange between droplets of oil/Triton X100/water emulsions, where the oil is p-xylene or octane, is reported. Combined with the toluene system data already reported in the previous chapters, this suggests that the process of oil transfer via oil permeation upon droplet collision or reversible coalescence of the droplets, independent of emulsion destabilization, may be a universal process. The increase of the time scale of the oil transfer, $\Delta_{\text {switch, }}$ as measured by PFG-NMR, from toluene to $p$-xylene to octane, emphasizes the significant role of the oil/emulsifier interactions in controlling the kinetics of the exchange process. 


\subsection{The microstructure of $p$-xylene/Triton $\mathrm{X}$ - 100/water emulsions}

Emulsions of the ternary system $p$-xylene/Triton X-100/water with an oil-tosurfactant weight ratio of 5:1 were prepared according to the same emulsification method used for the toluene system described in section 3.3. The nature of the continuous phase was confirmed by the solubilisation of a water soluble dye in the emulsion samples as well as the determination of the diffusion coefficient of the water phase using a PGSTE pulse sequence (see section 3.4.3.3); the $p$-xylene based emulsions were of the $\mathrm{O} / \mathrm{W}$ type between 2.5 and $70 \mathrm{wt} \%$ oil.

\subsubsection{Droplet size distribution}

The volume-based droplet size distributions of $p$-xylene/Triton X-100/water emulsions prepared with $p$-xylene between 2.5 and $70 \mathrm{wt} \%$ were measured by SLS (Figure 7.1) using a refractive index and an absorption coefficient of 1.390 and 0.001, respectively, and considering the droplets with irregular shapes.

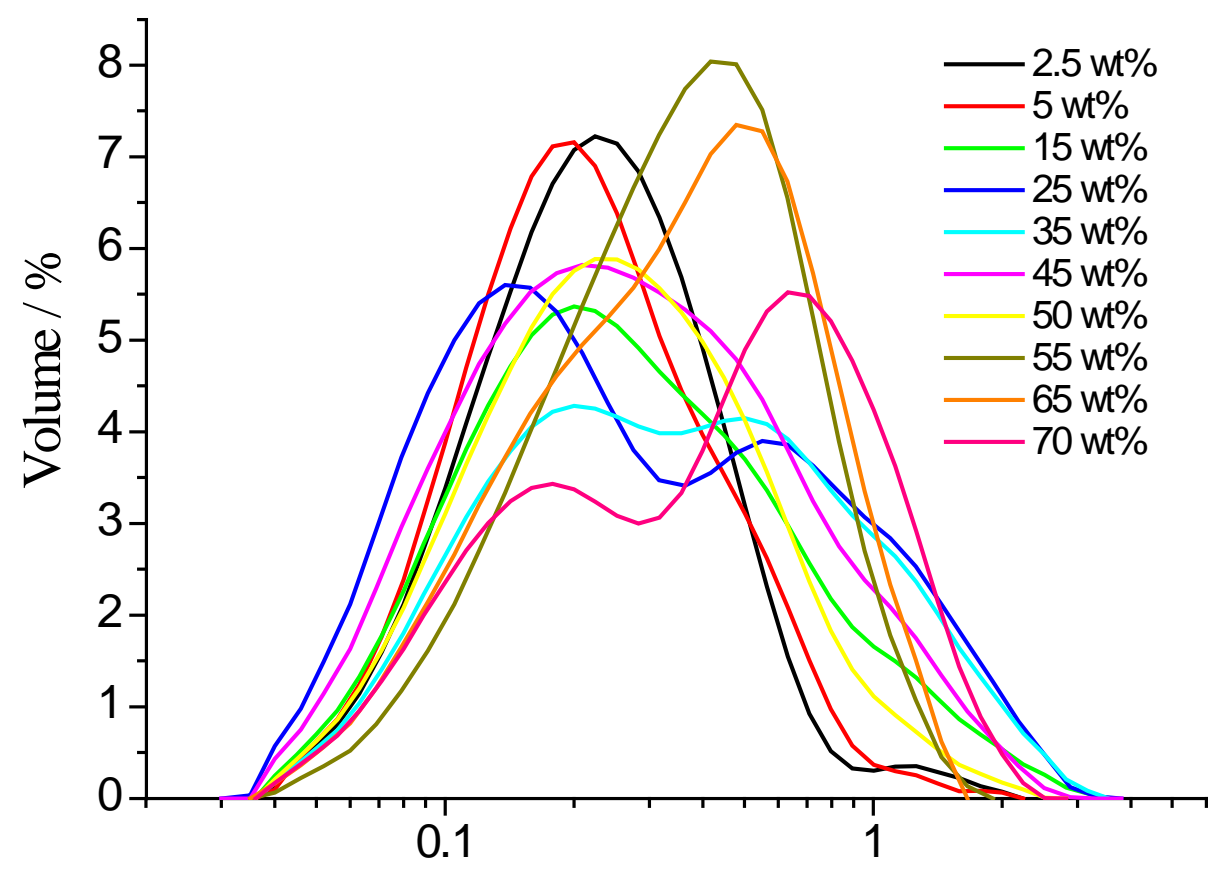

Droplet diameter / $\mu \mathrm{m}$

Figure 7.1. Droplet size distributions of emulsions between 2.5 and 70 wt $\%$ p-xylene using SLS. 
The droplet size distributions of the $p$-xylene emulsions indicate the presence of droplets measuring between $\sim 35 \mathrm{~nm}$ and $\sim 2$ to $\sim 3 \mu \mathrm{m}$ diameter, as for the toluene system. In contrast, however, the volume-based mean droplet size now depends on $p$-xylene concentration. For the toluene system the size distribution is independent of toluene concentration between 5 and 55 wt\% (Figure 4.1).

Upon increasing $p$-xylene concentration, the number of larger droplets from $\sim 0.3$ to $\sim 3 \mu \mathrm{m}$ diameter continuously increases at the expense of the number of smaller droplets from $\sim 35 \mathrm{~nm}$ to $\sim 0.25 \mu \mathrm{m}$ diameter leading to the increase of the volume-based mean droplet diameter (Table 7.1) and the distribution becoming occasionally bimodal. It is hypothesized that the two modes are always present however it is not always possible to resolve these two close peaks, as such the size distribution appears either bimodal with two distinct maxima (e.g. $25 \mathrm{wt} \%$ pxylene, Figure 7.1) or monomodal with a maximum value being the average of two maxima (e.g. $45 \mathrm{wt} \%$ p-xylene, Figure 7.1). This limited ability to resolve the two modes results in the calculated volume-based mean droplet diameters being under estimated from 40 to $65 \mathrm{wt} \%$ oil (Table 7.1).

Comparing the $p$-xylene and toluene systems with respect to the microstructure, the volume-based mean droplet size of $p$-xylene emulsions ranges from $0.27 \pm$ 0.10 to $0.53 \pm 0.10 \mu \mathrm{m}$ upon increasing oil concentration from 2.5 to $70 \mathrm{wt} \%$. This is $\sim 14$ to $138 \%$ larger than that of the toluene emulsions (Table 7.1) and is additionally a function of oil concentration. 


\begin{tabular}{|c|c|c|}
\hline Oil concentration / wt\% & $\begin{array}{l}\text { Volume-mean } p \text {-xylene } \\
\text { droplet diameter } \\
\pm 0.10 / \mu \mathrm{m}\end{array}$ & $\begin{array}{l}\text { Volume-mean toluene } \\
\text { droplet diameter } \\
\pm 0.10 / \mu \mathrm{m}\end{array}$ \\
\hline 2.5 & 0.27 & \\
\hline 5 & 0.27 & 0.18 \\
\hline 10 & 0.44 & 0.21 \\
\hline 15 & 0.39 & 0.28 \\
\hline 25 & 0.45 & 0.24 \\
\hline 30 & 0.50 & 0.21 \\
\hline 35 & 0.51 & 0.25 \\
\hline 40 & 0.30 & 0.18 \\
\hline 45 & 0.39 & 0.24 \\
\hline 50 & 0.33 & 0.29 \\
\hline 55 & 0.42 & 0.22 \\
\hline 60 & 0.45 & 0.37 \\
\hline 65 & 0.42 & 0.36 \\
\hline 70 & 0.53 & 0.37 \\
\hline
\end{tabular}

Table 7.1. SLS volume-based mean droplet diameter for emulsions prepared with $p$-xylene or toluene between 2.5 and $70 \mathrm{wt} \%$.

The p-xylene emulsions also contain oil swollen micelles dispersed in the aqueous continuous phase as evidenced by the DLS measurements (Figure 7.2) performed on the expelled aqueous bottom phase of a separated aged emulsion (the refractive index and absorption coefficient of the oil phase were taken as 1.390 and 0.001 , respectively). Micelles span from $\sim 6$ to $\sim 50 \mathrm{~nm}$ with a mean diameter of $18 \pm 5 \mathrm{~nm}$ for emulsions prepared with 20, 35 and $40 \mathrm{wt} \%$-xylene, in similarity to the size distributions measured for the toluene system (Figure 4.4). 


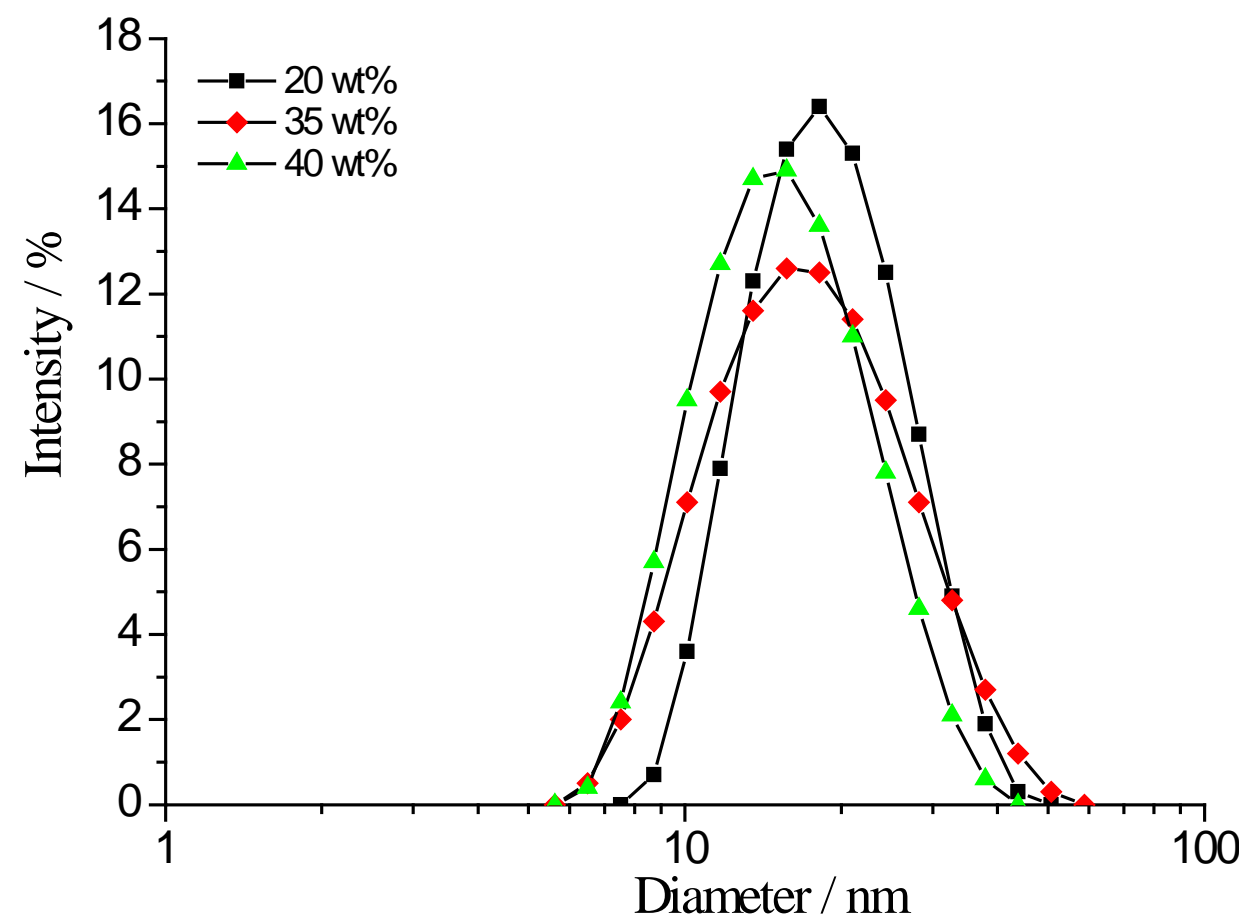

Figure 7.2. Size distributions of the expelled aqueous phase of aged emulsions prepared with 20, 35 and $40 \mathrm{wt} \%$ p-xylene using DLS.

\subsubsection{Macroscopic phase separation}

The macroscopic phase separation data for emulsions prepared with $p$-xylene between 2.5 and $70 \mathrm{wt} \%$ are depicted by the plot of the amount of expelled aqueous phase as a function of time (Figure 7.3, not all concentrations are shown for clarity). As for the toluene system, concentration domains are differentiated according to the time of the beginning of the phase separation, the time of the initial fast phase separation (steep increase in the amount of expelled phase) and the time, $t_{\text {static }}$, at which the amount of expelled phase remains nearly constant.

The $p$-xylene system comprises five concentration domains: 2.5 to $5 \mathrm{wt} \%, 10$ to $20 \mathrm{wt} \%, 25$ to $35 \mathrm{wt} \%, 40$ to $55 \mathrm{wt} \%$ and 65 to $70 \mathrm{wt} \%$ oil with transition concentrations being observed for 7.5, 23 and $60 \mathrm{wt} \%$ oil. That is, one additional domain (2.5 to $5 \mathrm{wt} \%$ oil) is evident compared with the toluene system (Figure 6.1). 


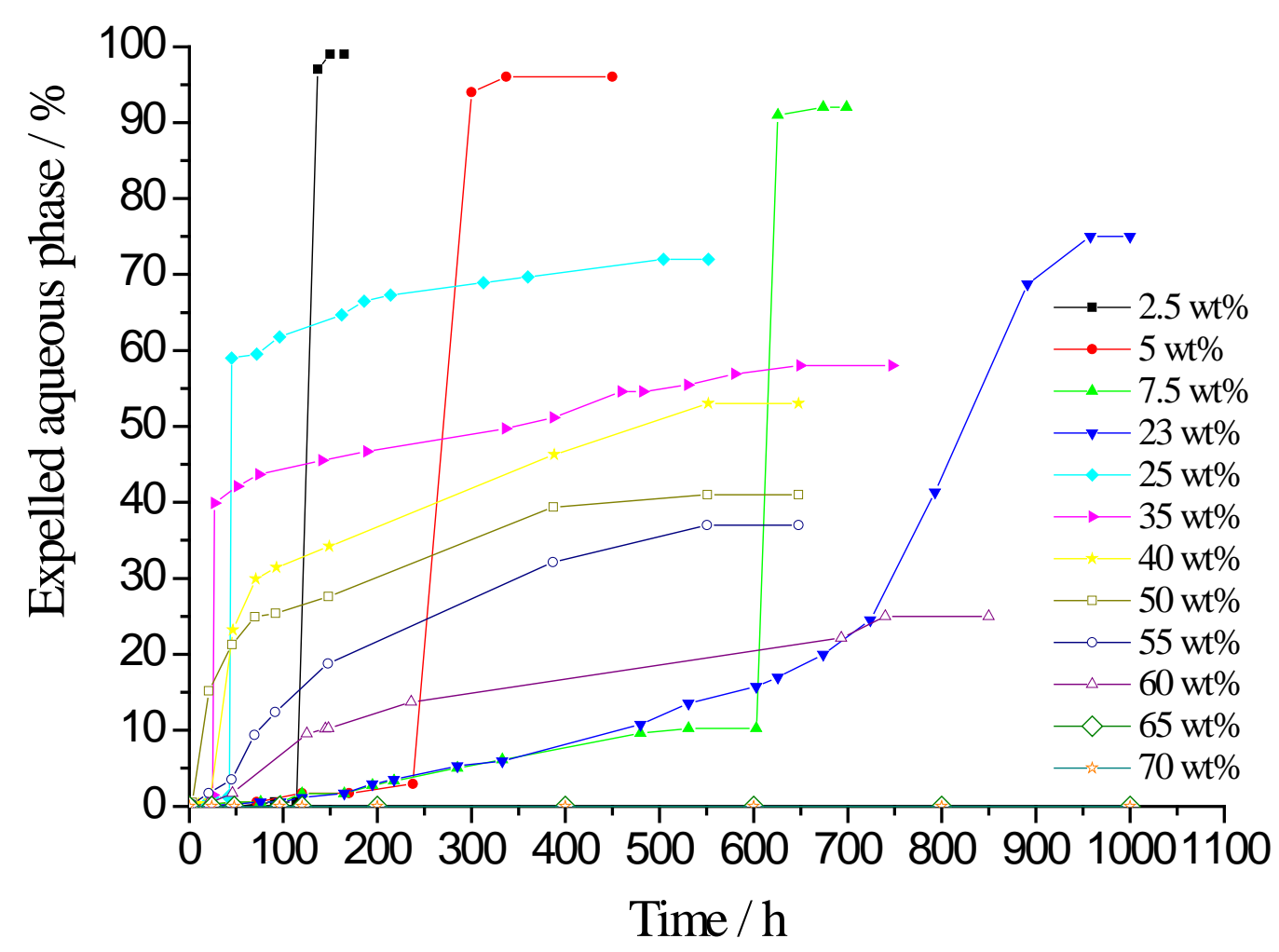

Figure 7.3. Amount of expelled aqueous phase as a function of time for $p$-xylene/Triton $X$ 100/water emulsions prepared with $p$-xylene between 2.5 and $70 \mathrm{wt} \%$.

The first of these concentration domains occurs at very low oil concentrations, between 2.5 and $5 \mathrm{wt} \%$ p-xylene. In this region, the time to reach the same extent of phase separation associated to a specific mean droplet size, e.g. the time of the initial rapid phase separation or $\mathrm{t}_{\text {static }}$, is an increasing function of oil concentration (Figure 7.4 and Table 7.2) despite increasing the droplet collision frequency as the size distribution remains nearly unchanged (Figure 7.1and Table 7.1).

Between 7.5 and $23 \mathrm{wt} \%$ p-xylene, the plots of the amount of expelled aqueous phase versus time (Figure 7.5) follow a much slower separation trend compared to the other $p$-xylene concentrations (Figure 7.3). Considering the plot of the volume fraction of expelled aqueous phase normalized to the extent of phase separation at $t_{\text {static }}$ for $p$-xylene concentrations between 10 and $20 \mathrm{wt} \%$ (Figure 7.6) in comparison with the corresponding low concentration region of the toluene system (Figure 6.4), the phase separation process occurs on a much slower time scale. Here, $p$-xylene droplets are more stable with respect to emulsion destabilization with phase separation being completed within 690 to $\sim 800 \mathrm{~h}$ (Table 7.2) as compared with 186 to $336 \mathrm{~h}$ in the toluene system (Table 6.2). Moreover, these $p$-xylene emulsions follow similar phase separation 
behaviours, as seen by the times of both the beginning of phase separation and the initial fast phase separation being constant (Figure 7.6 and Table 7.2), whereas in the toluene system a variation with increasing oil concentration is evident.

From 25 to $35 \mathrm{wt} \%$ ( $3^{\text {rd }}$ concentration domain) and from 40 to $55 \mathrm{wt} \% p$-xylene ( $4^{\text {th }}$ concentration domain) (Figure 7.7 and Figure 7.8), although emulsions begin to phase separate faster ( 2.5 to $\sim 25 \mathrm{~h}$, Table 7.2 ) than the corresponding samples in the toluene system ( $\sim 7$ to $\sim 35 \mathrm{~h}$, Table 6.1 and Table 6.3$)$, the overall macroscopic process spanning from $\sim 504$ to $\sim 649 \mathrm{~h}$ (Table 7.2) takes more time than in the toluene system ( 318 to $\sim 480 \mathrm{~h})$. This indicates that the mean droplet size of the $p$-xylene emulsions remains larger than that of the toluene emulsions within the first few hours following emulsification for the more concentrated samples, resulting in the observed shorter times for the start of phase separation. However, the longer rate of droplet growth leading to a longer destabilization process measured in the $p$-xylene system for all concentrations compared to the toluene system is controlled by the properties of the interfacial membrane which are vastly different in the two oil systems.

Finally, the 65 and $70 \mathrm{wt} \% p$-xylene emulsions do not phase-separate prior to $\sim 1$ month after emulsification (Figure 7.3) which makes them more stable than the corresponding samples in the toluene system which start to destabilize by $8 \mathrm{~h}$ and 18 days, respectively. 


$\left.\begin{array}{|c|c|c|c|}\hline \begin{array}{c}\text { Oil } \\ \text { concentration } \\ \text { / wt\% }\end{array} & \begin{array}{c}\mathrm{t}_{\text {start of phase }} \\ \text { separation } / \mathrm{h}\end{array} & \begin{array}{c}\mathrm{t}_{\text {initial fast phase }} \\ \text { separation } / \mathrm{h}\end{array} & \mathrm{t}_{\text {static }} / \mathrm{h} \\ \hline 2.5 & 91 & 137 & 150 \\ \hline 3.75 & 68 & 240 & 285 \\ \hline 5 & 46 & 300 & 337\end{array}\right\}$ Region 1

Table 7.2. Characteristic times of the macroscopic phase separation process for the four concentration domains and the three transition concentrations of the $p$-xylene/Triton $X$ 100/water system between 2.5 and 60 wt \% p-xylene. 


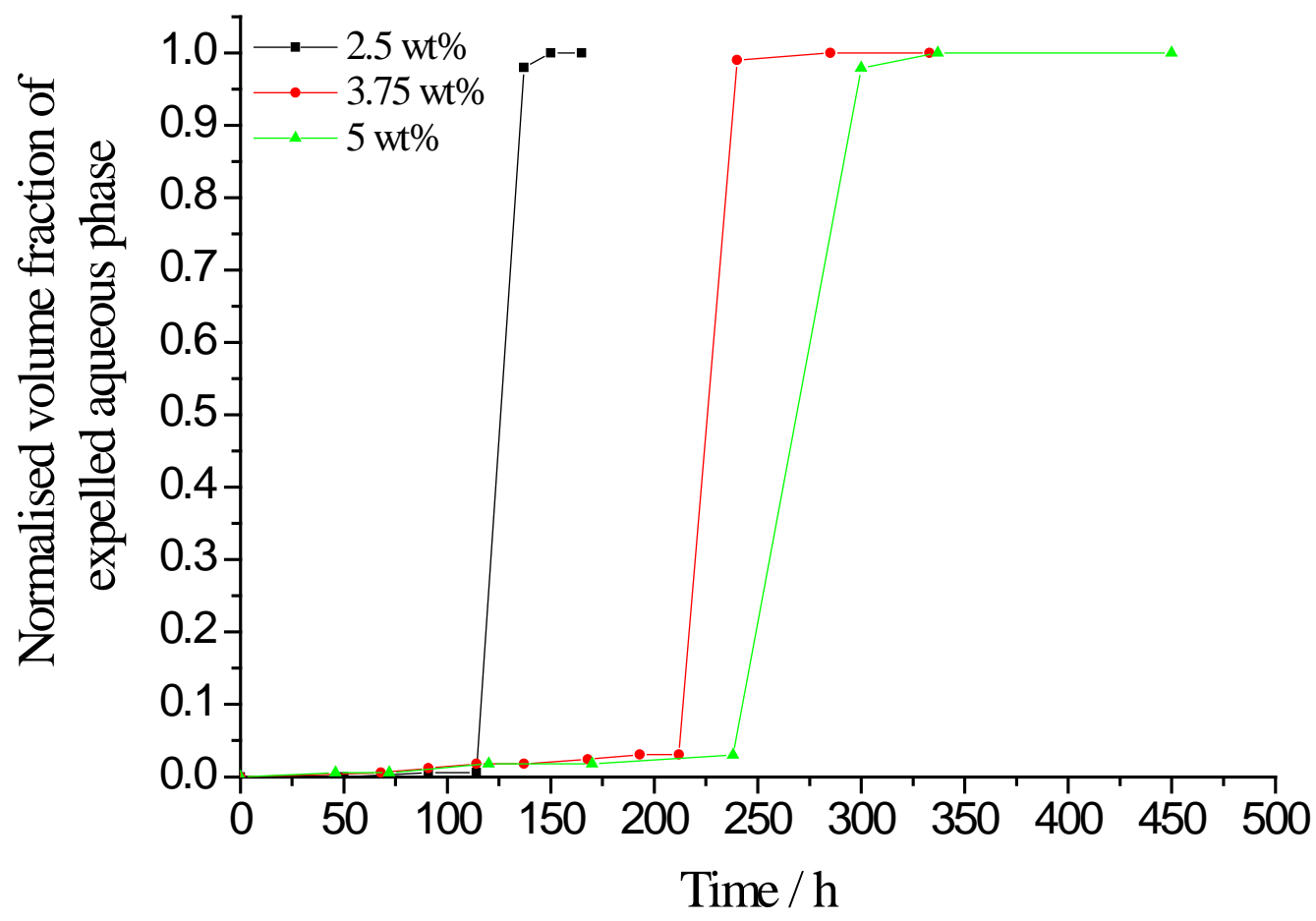

Figure 7.4. Volume fraction of expelled aqueous phase normalised to the limiting value measured at $t_{\text {static }}$ as a function of time for $p$-xylene/Triton $\mathrm{X}-100 /$ water emulsions prepared with $p$-xylene between 2.5 and 5 wt\%.

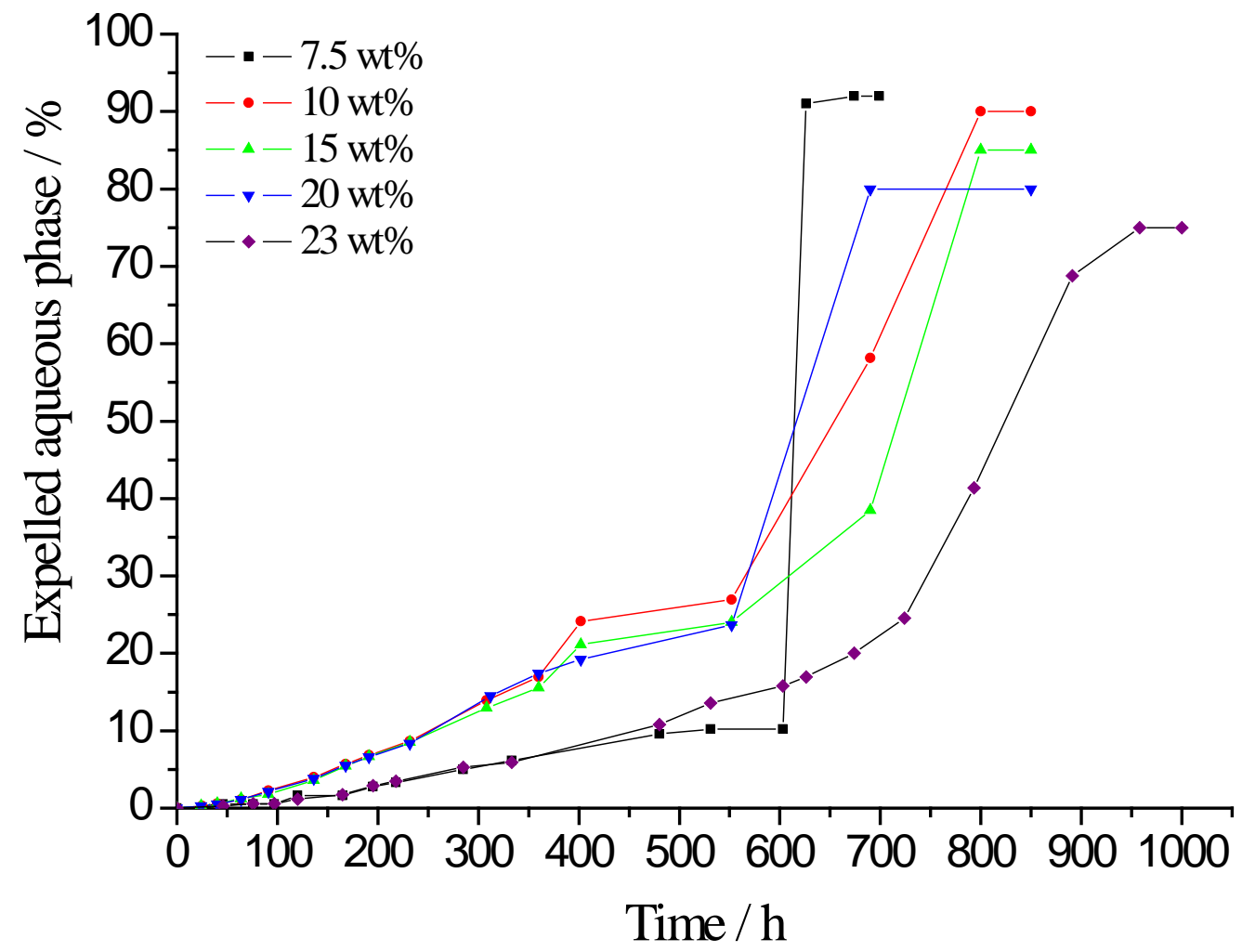

Figure 7.5. Amount of expelled aqueous phase as a function of time for $p$-xylene/Triton X100/water emulsions prepared with $p$-xylene between 7.5 and $23 \mathrm{wt} \%$. 


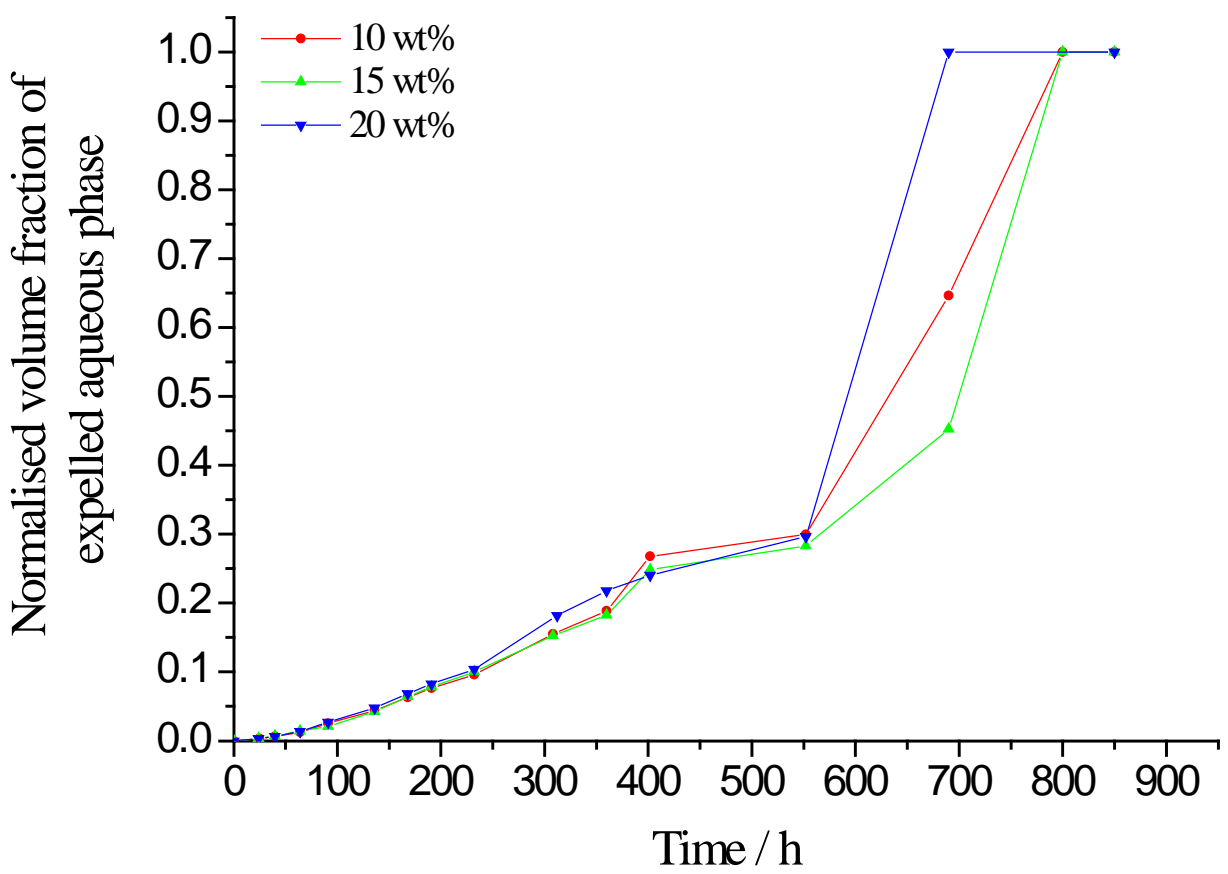

Figure 7.6. Volume fraction of expelled aqueous phase normalised to the limiting value measured at $t_{\text {static }}$ as a function of time for $p$-xylene/Triton $\mathrm{X}-100 /$ water emulsions prepared with $p$-xylene between 10 and 20 wt \% .

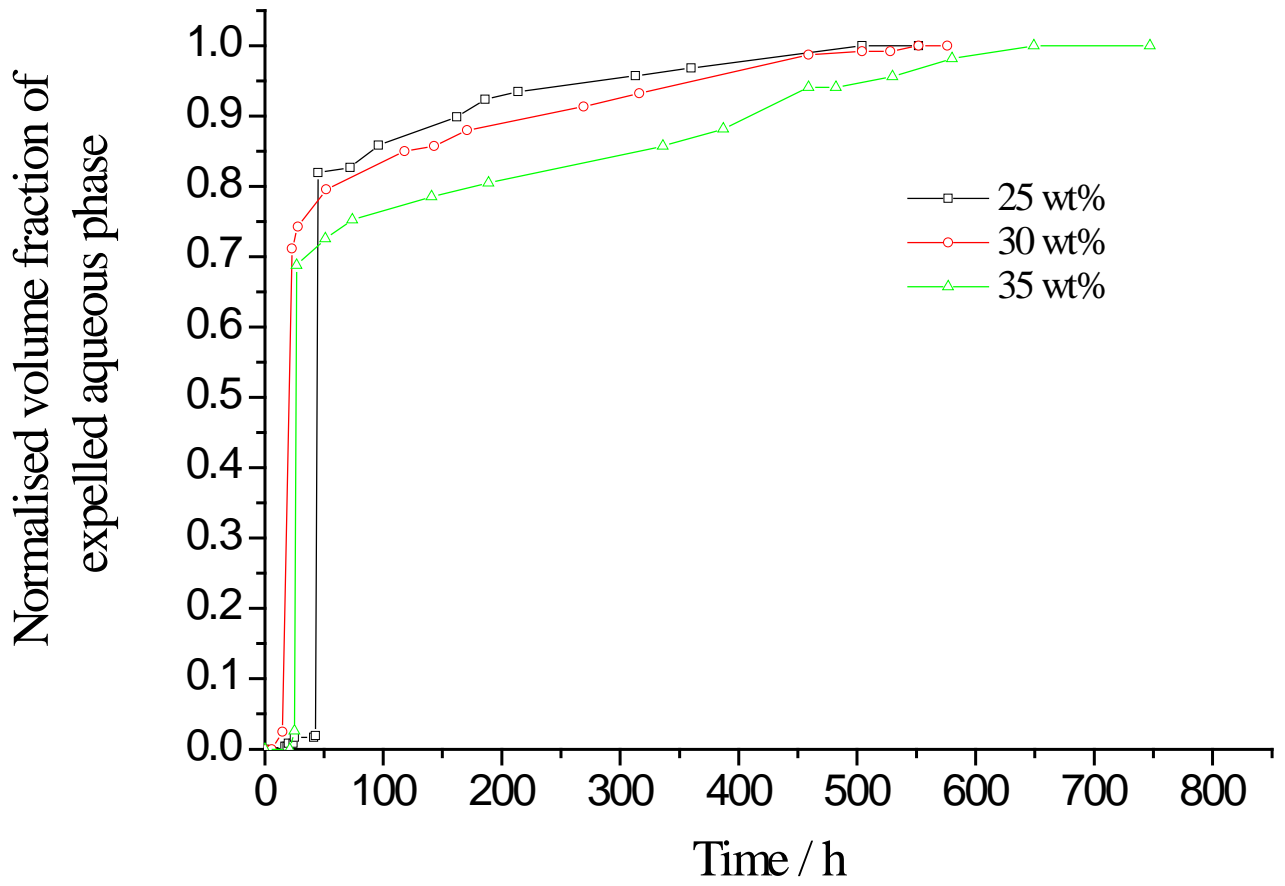

Figure 7.7. Volume fraction of expelled aqueous phase normalised to the limiting value measured at $t_{\text {static }}$ as a function of time for $p$-xylene/Triton $\mathrm{X}-100 /$ water emulsions prepared with $p$-xylene between 25 and 35 wt \%. 


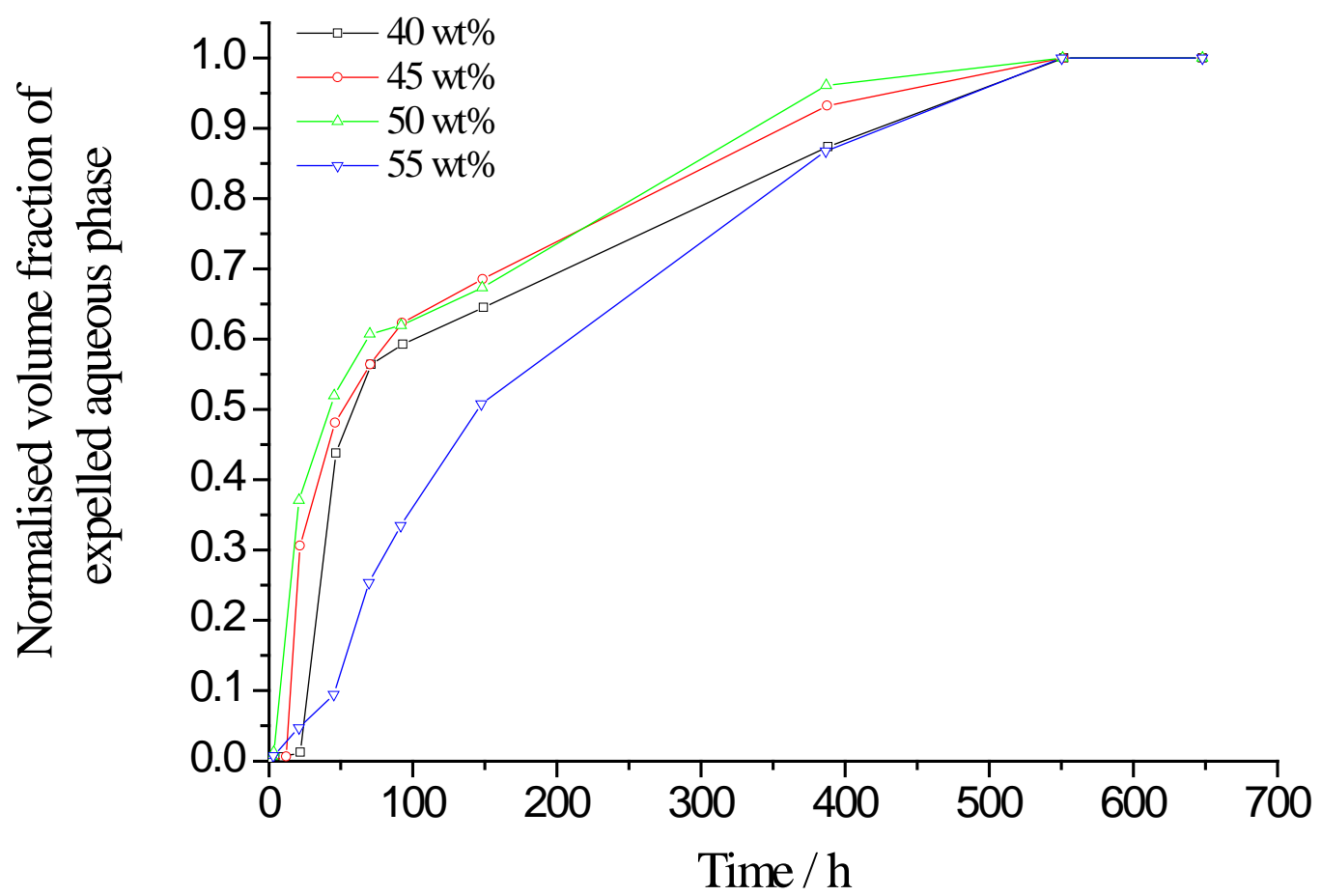

Figure 7.8. Volume fraction of expelled aqueous phase normalised to the limiting value measured at $t_{\text {static }}$ as a function of time for $p$-xylene/Triton $X-100 /$ water emulsions prepared with $p$-xylene between 40 and 55 wt\%.

In order to assess how the oil exchange process probed in toluene emulsions is affected by the nature of the oil, laser scanning confocal microscopy and cryoSEM experiments were performed on oil/Triton X-100/water emulsions where the oil is $p$-xylene or octane. 


\subsection{Effect of the oil on the dynamics of oil transfer}

\subsubsection{Laser scanning confocal microscopy}

Upon replacing toluene with either $p$-xylene or octane (note that $\mathrm{O} / \mathrm{W}$ emulsions can only be formed between 40 and $60 \mathrm{wt} \%$ oil in the octane system [133]), oil exchange between dispersed droplets with no concomitant variation of the droplet size still occurs as probed by laser scanning confocal microscopy. After mixing separately prepared red and green fluorescing emulsions according to the experimental protocol detailed in section 4.3, all oil droplets emit yellow fluorescence resulting from the solubilisation of the two dyes in the same oil environment within $\sim 10$ min of mixing and $\sim 30$ min after emulsification (Figure 7.9 and Figure 7.10).

After mixing the two individually labelled emulsions in both the p-xylene and octane systems, the droplet size distribution remains unchanged and corresponds well to the SLS data for freshly emulsified samples with or without dye addition. In the 2D confocal images of the $65 \mathrm{wt} \%$-xylene emulsions (Figure 7.9), most of the droplets are between $\sim 0.6$ and $\sim 1 \mu$ m diameter (note that the microscope resolution is $\sim 200 \mathrm{~nm}$ ), which fits reasonably well the SLS data (Figure 7.1). The optical cross-sections for the $40 \mathrm{wt} \%$ octane emulsions (Figure 7.10) indicate the presence of droplets measuring $\sim 0.6$ to $\sim 1.3 \mu \mathrm{m}$ diameter, which is consistent with the SLS data presented by Liu and coworkers (Figure 4 in the reference [133]). 

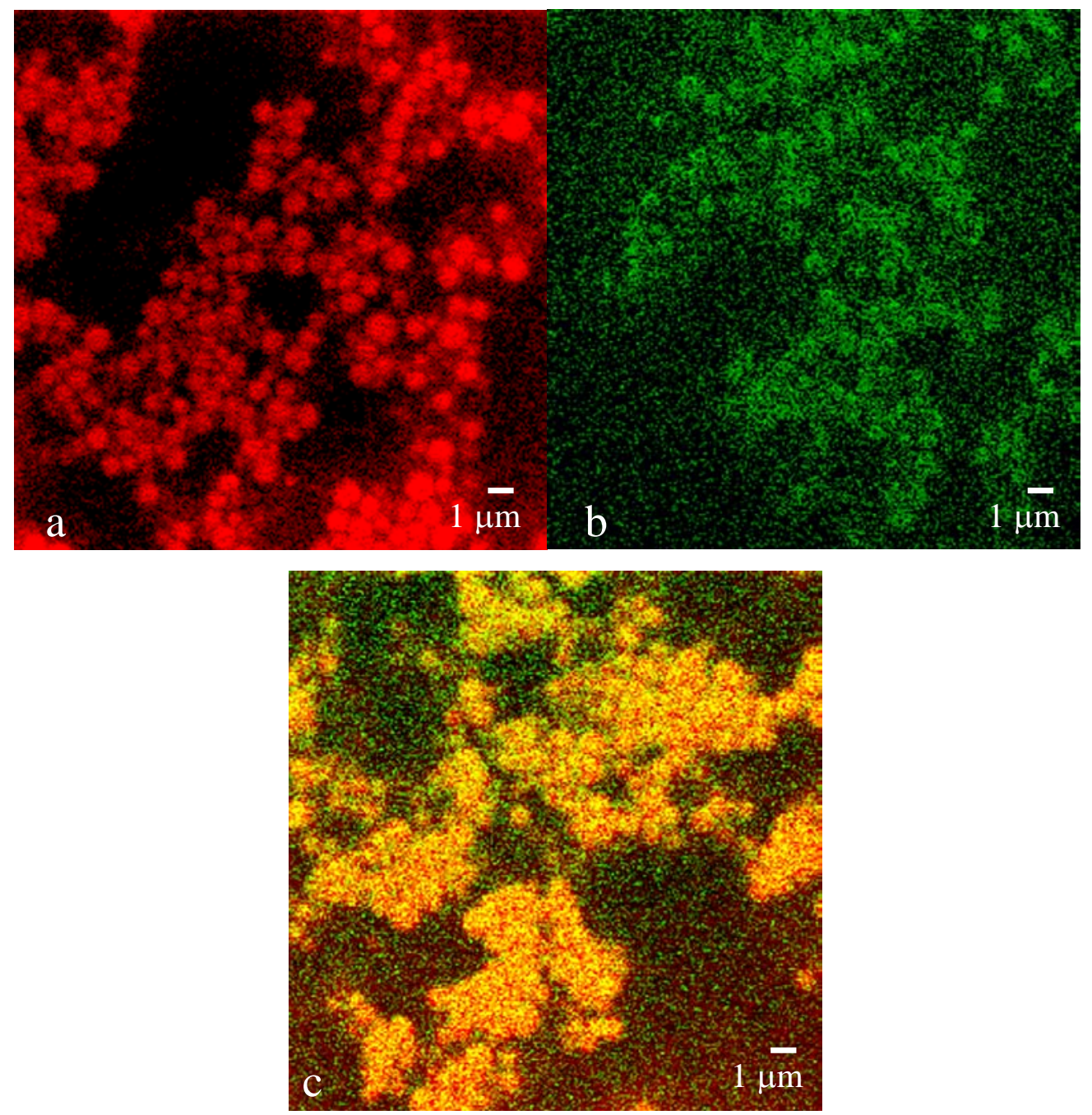

Figure 7.9. 2D confocal microscopy optical cross-sections $256 \times 256(\times 100$ objective, zoom factor 4) of fresh $65 \mathrm{wt} \% p$-xylene emulsions labelled with Nile red (a) and 4-(4methoxybenzylamino)-7-nitrobenzofurazan (b). Snapshot (c) results from the mixing of the red fluorescing (a) and the green fluorescing (b) emulsions. 

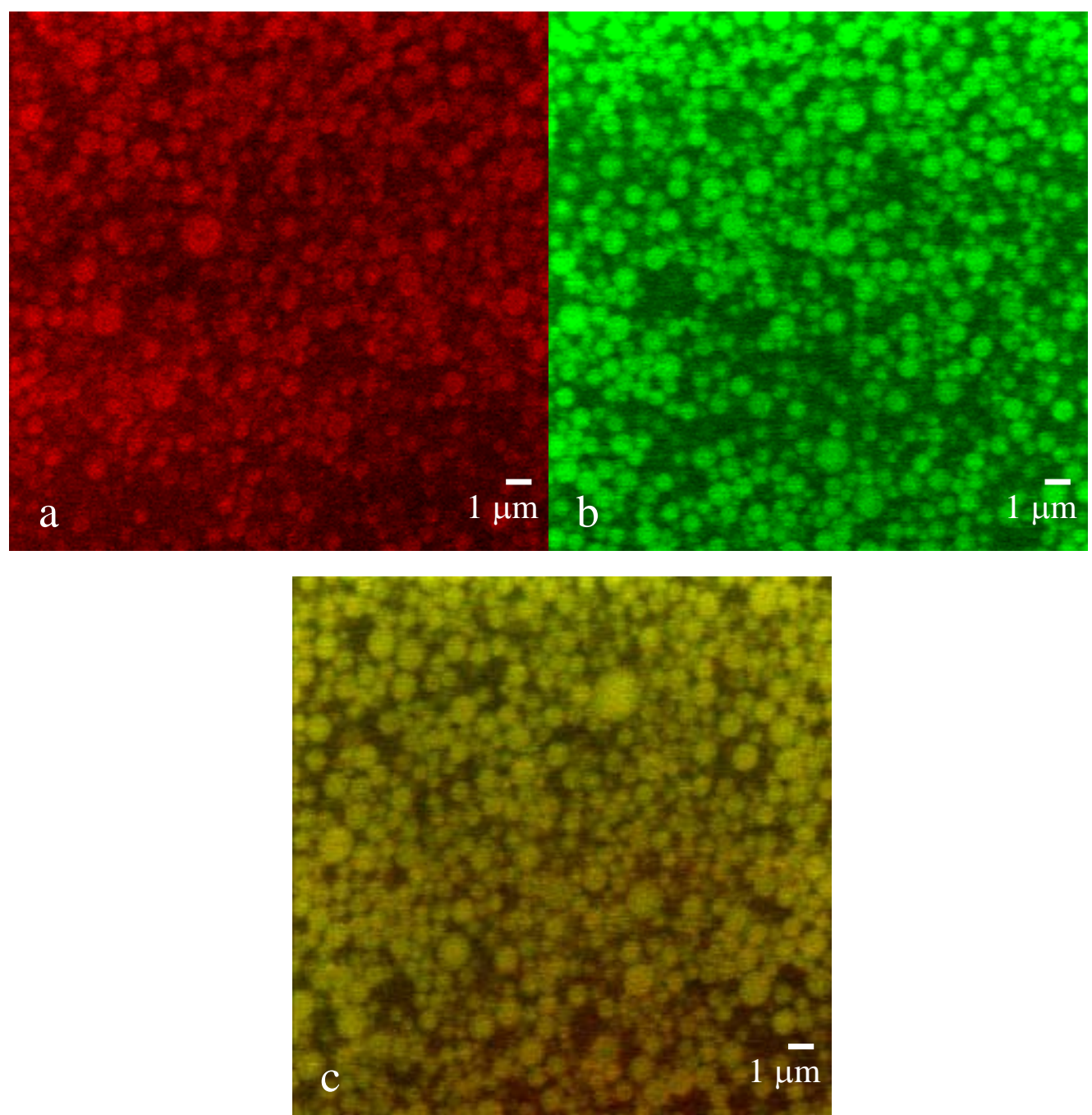

Figure 7.10. 2D confocal microscopy optical cross-sections $256 \times 256(\times 100$ objective, zoom factor 4) of fresh $40 \mathrm{wt} \%$ octane emulsions labelled with BODIPY 665/676 (a) and BODIPY $493 / 503$ (b). Snapshot (c) results from the mixing of the red fluorescing (a) and the green fluorescing (b) emulsion.

The confocal microscopy data provide evidence that oil exchange between emulsion droplets that does not lead to droplet growth, occurs in the three ternary systems: oil/Triton X-100/water where the oil is one of toluene, $p$-xylene or octane. However, this technique does not highlight any effect of the nature of the oil on the dynamics of oil transfer. As such, cryo-SEM was performed on $p$ xylene and octane emulsions in order to examine the internal microstructure on a faster experimental time scale, i.e. the freezing time was $\sim 1 \mathrm{~s}$. 


\subsubsection{Cryo-SEM}

The cryo-SEM micrographs presented in Figure 7.11 to Figure 7.13 for fresh and aged $40 \mathrm{wt} \%$ and aged $15 \mathrm{wt} \%$-xylene emulsions indicate significant droplet interaction within the SEM experimental time scale, i.e. the freezing time. Evident is the formation of a network of partially coalesced droplets irrespective of the age of the emulsion. As it is the case for the toluene system, this network of $p$ xylene droplets is not representative of the droplet size distribution measured by SLS (Figure 7.1 for fresh samples) and cannot be the result of irreversible coalescence given the stability of the samples (the 15 and $40 \mathrm{wt} \%$-xylene emulsions start to phase-separate only after $\sim 24$ and $\sim 6 \mathrm{~h}$, respectively, Table 7.2).

Consider the cryo-SEM images for a freshly emulsified $40 \mathrm{wt} \%$ octane sample as shown in Figure 7.14 and Figure 7.15. The internal microstructure of the emulsion is strikingly different to that of both the toluene and $p$-xylene systems. Within the $\sim 1 \mathrm{~s}$ freezing time, octane droplets maintain their integrity and there is no indication of any direct contact or formation of a network of interconnected droplets. This suggests that oil transfer in the octane system occurs on a much longer time scale than in either the $p$-xylene or toluene systems, though still faster than $\sim 10$ min as evidenced from the confocal microscopy data. 


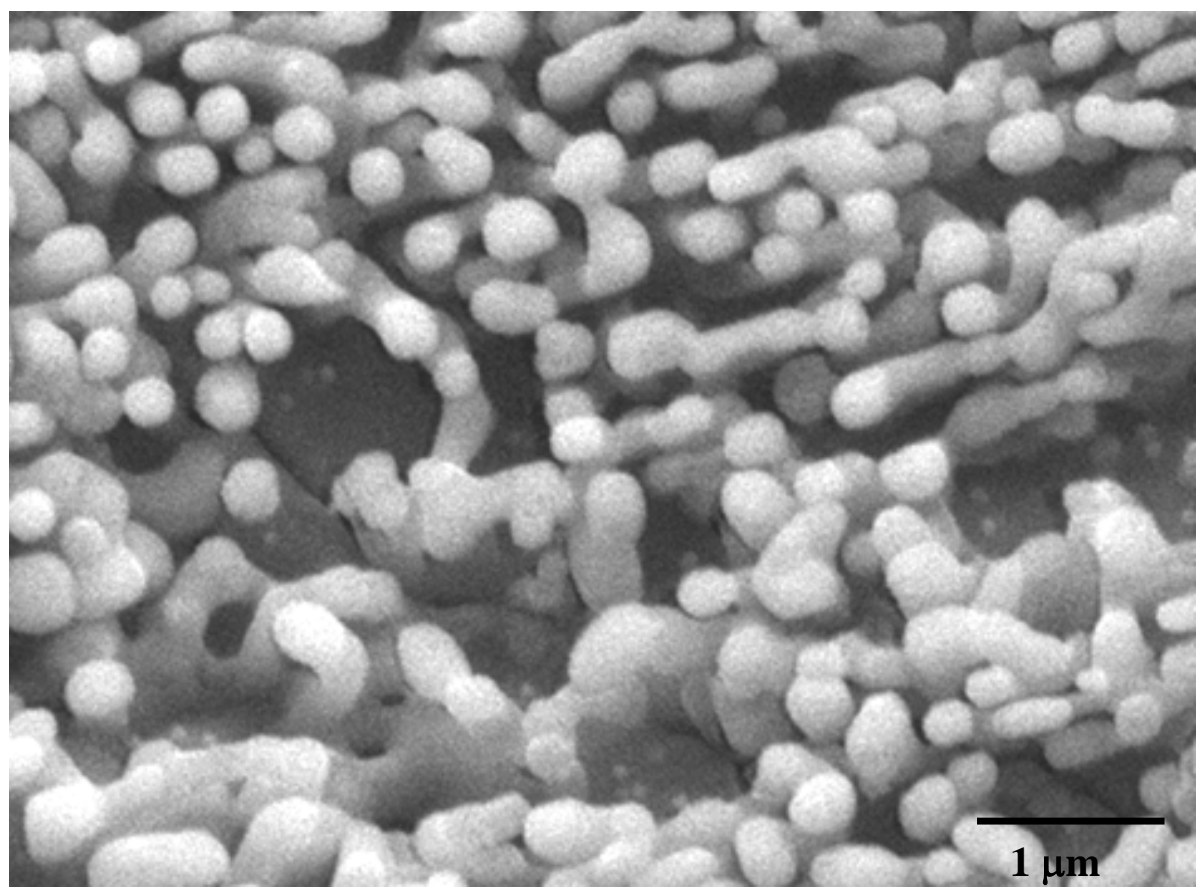

Figure 7.11. Cryo-SEM image of a freshly emulsified $40 \mathrm{wt} \%$ p-xylene emulsion (×20000).

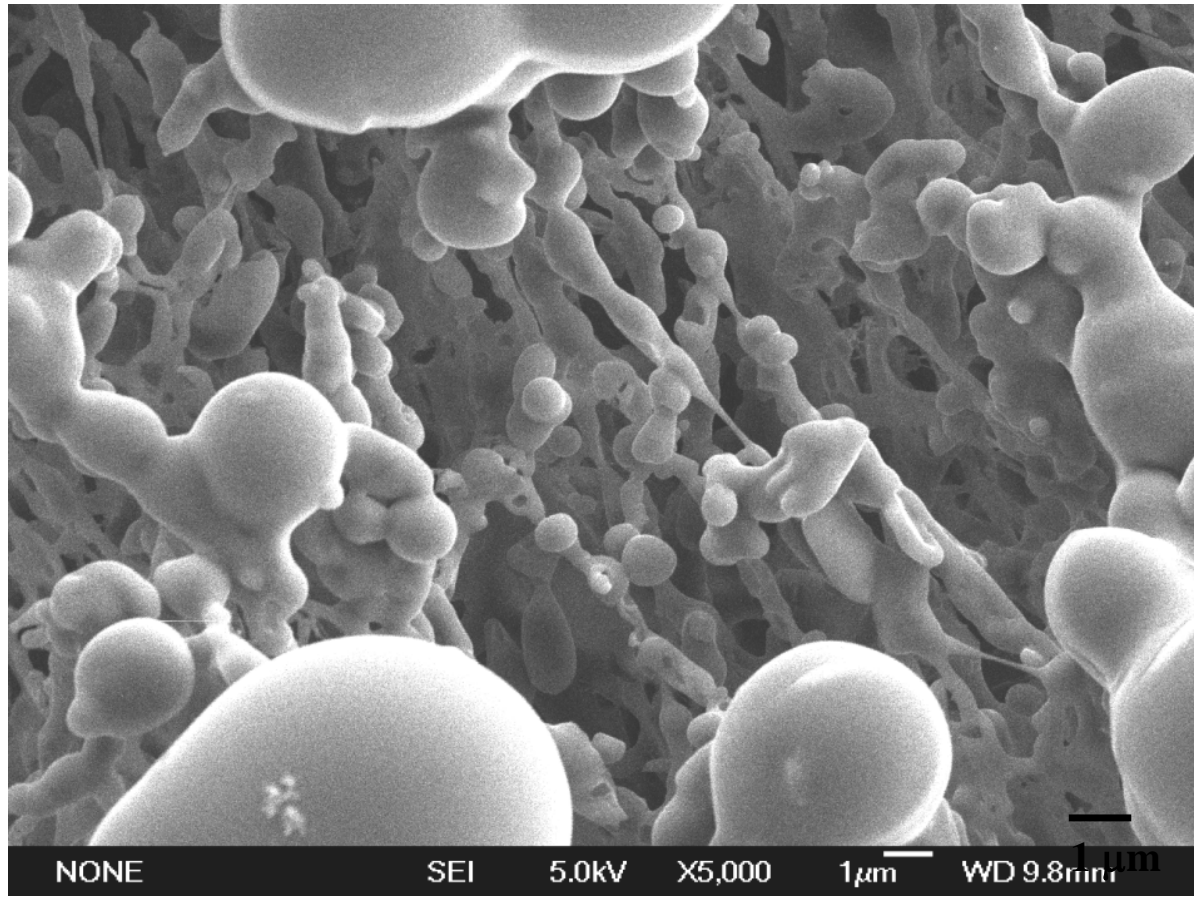

Figure 7.12. Cryo-SEM image of a $15 \mathrm{wt} \% \mathrm{p}$-xylene emulsion aged for $4 \mathrm{~h}(\times 5000)$. 


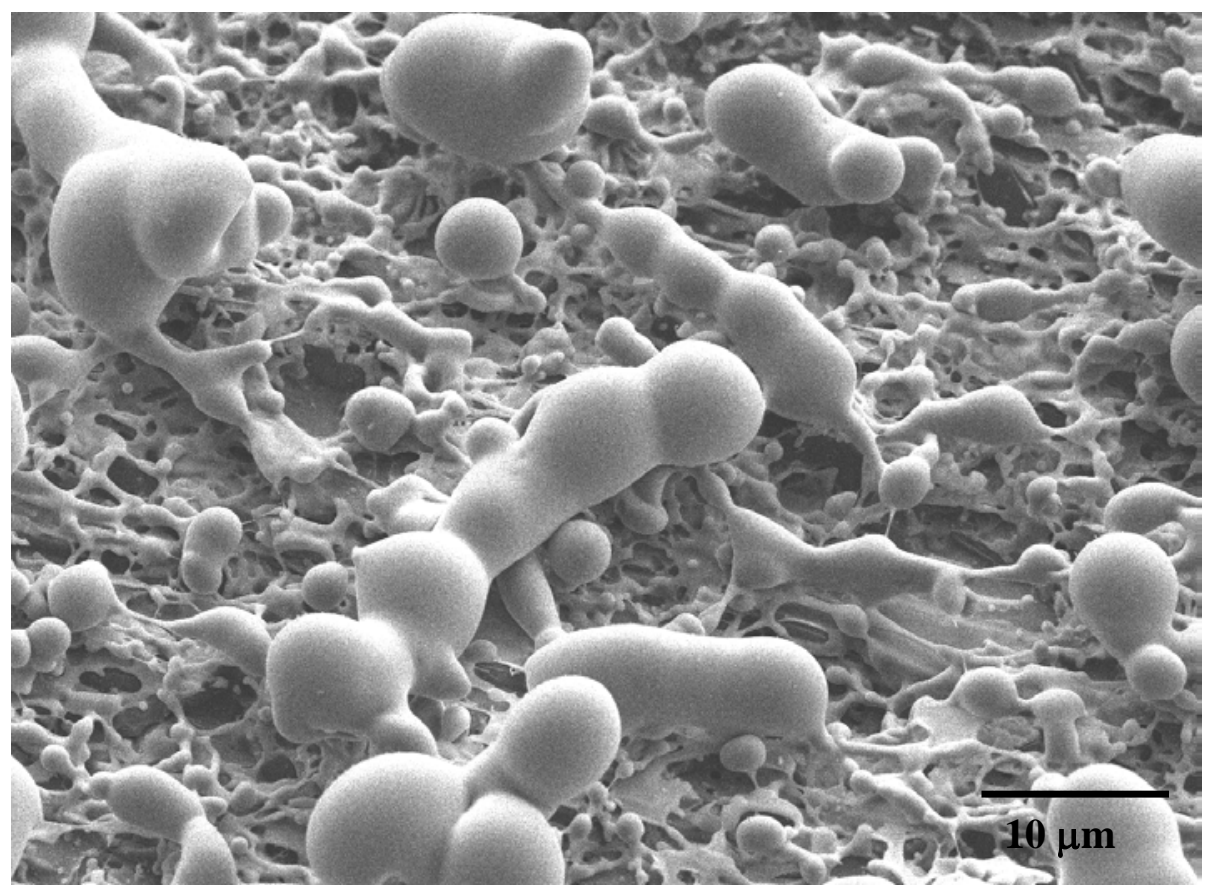

Figure 7.13. Cryo-SEM image of a $40 \mathrm{wt} \% p$-xylene emulsion aged for 9 days $(\times 2000)$.

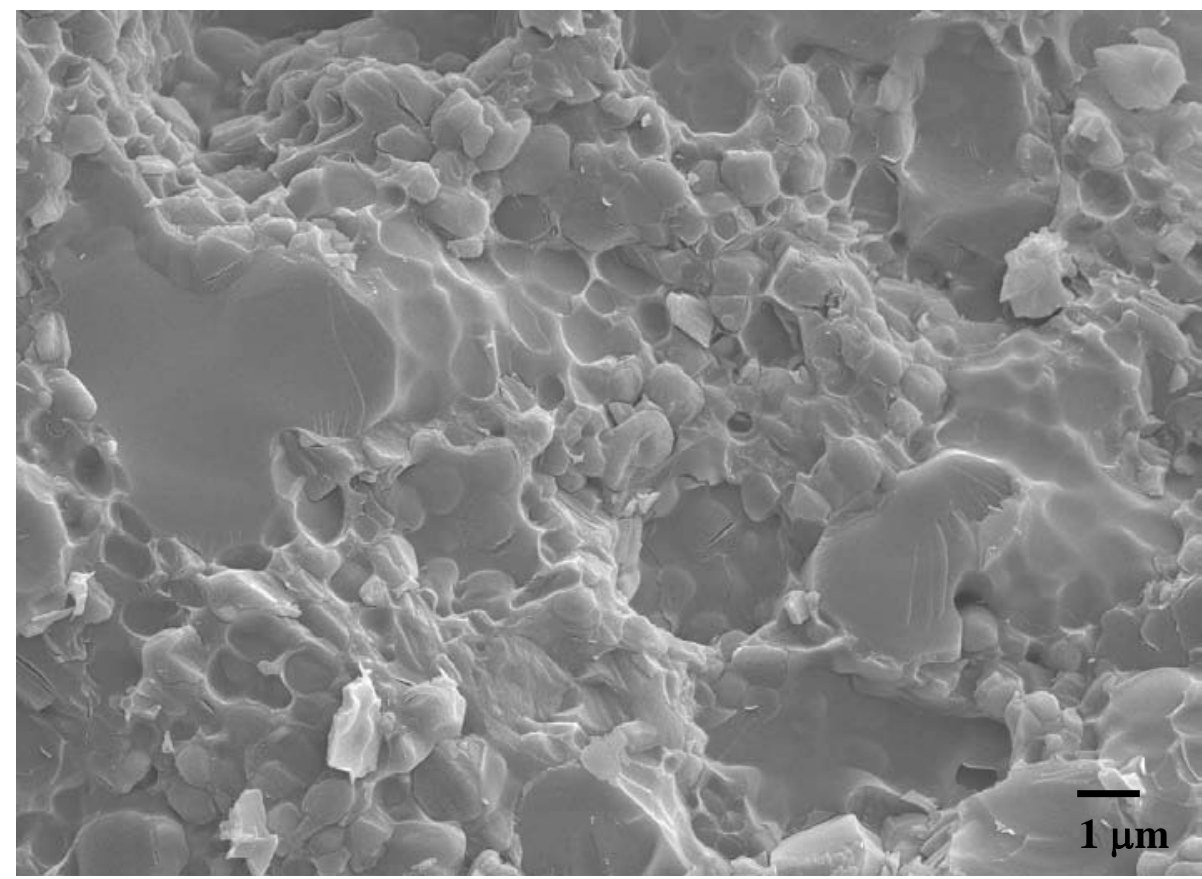

Figure 7.14. Cryo-SEM image of a freshly emulsified $40 \mathrm{wt} \%$ octane emulsion $(\times 5000)$. 


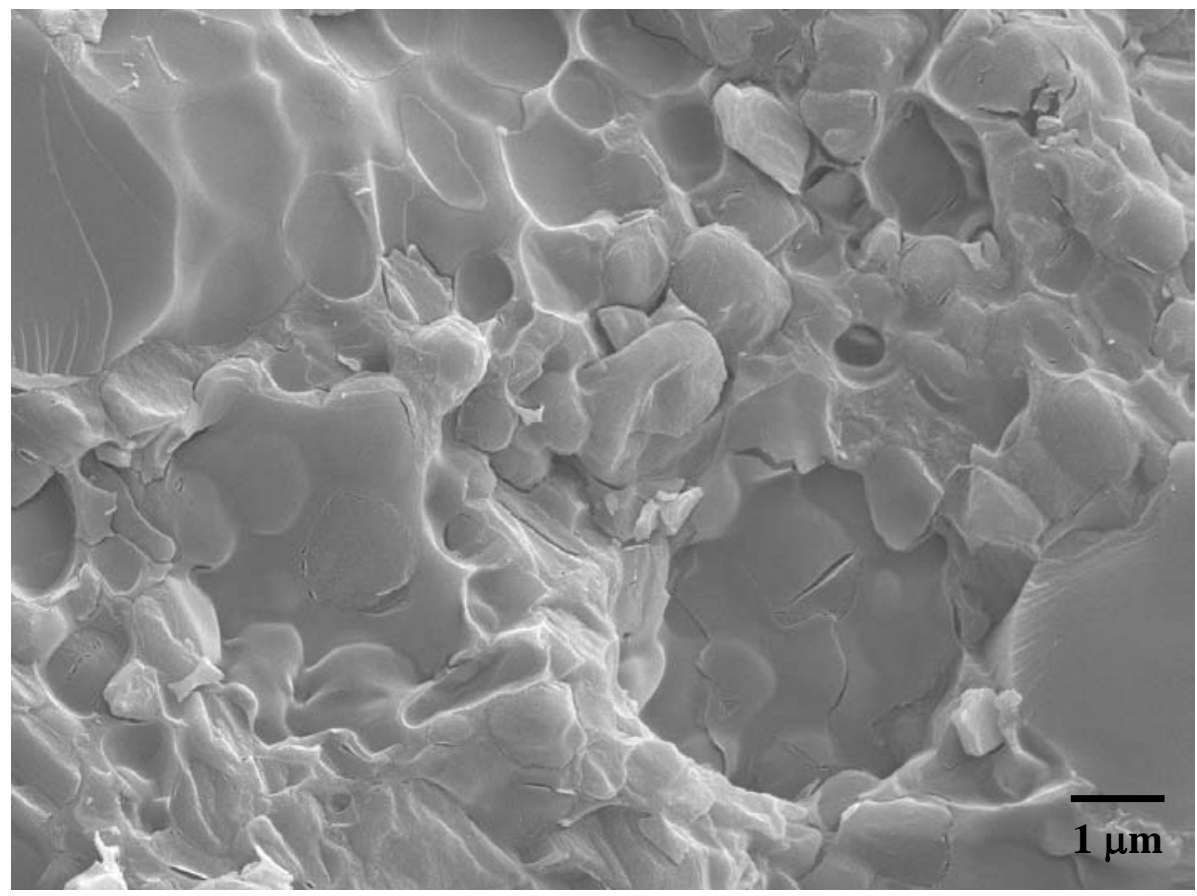

Figure 7.15. Cryo-SEM image of a freshly emulsified $40 \mathrm{wt} \%$ octane emulsion $(\times 10000)$.

\subsubsection{PFG-NMR}

The microstructure and dynamics of the oil phase in $p$-xylene emulsions were monitored upon varying the NMR observation time, $\Delta$, using a PGSTE pulse sequence (see section 3.4.3.3). The switch between a single exponential decay as a function of gradient strength, where motional averaging of the oil diffusion is measured, and a multiexponential decay, where the motions of droplets and oil molecules are probed, upon decreasing $\Delta$ occurs at $\Delta_{\text {switch }}$ defined as the time scale of the oil exchange.

\subsubsection{Microstructure}

The diffusion echo attenuations of the oil signal for toluene and $p$-xylene emulsions prepared with, for example $40 \mathrm{wt} \%$ oil (Figure 7.16), may be directly compared. At long observation times, $\Delta=1.3 \mathrm{~s}$, for toluene and $\Delta=1.5 \mathrm{~s}$ for $p$ xylene, i.e. where motional averaging is observed, the fast contribution, associated with the unrestricted diffusion of oil molecules dissolved directly in the aqueous continuous phase, is more important in toluene as compared with $p$ - 
xylene emulsions. This observation is consistent with the lower solubility of $p$ xylene in water compared to toluene, hence decreasing the volume fraction of oil molecules dissolved directly in the water phase.

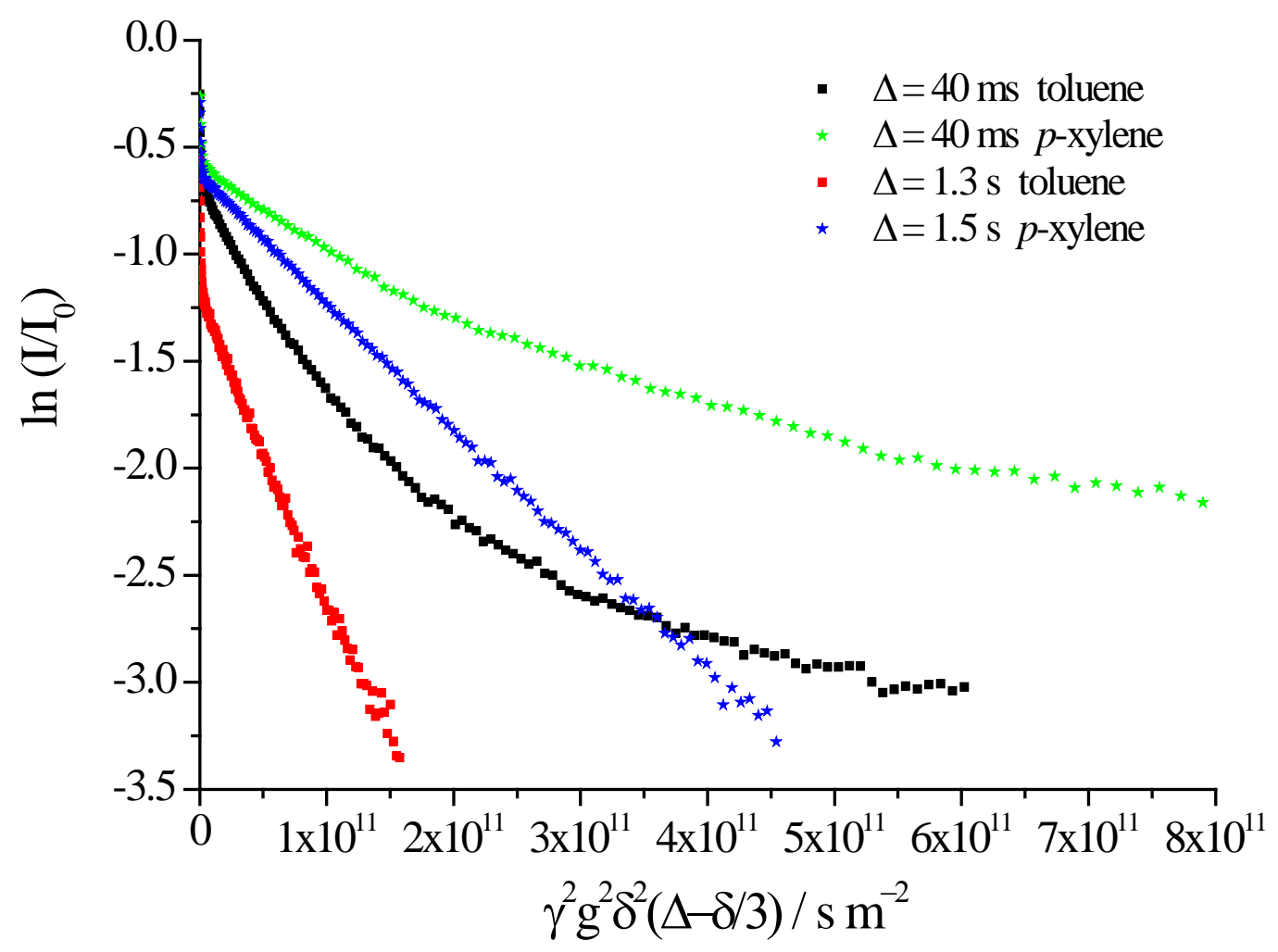

Figure 7.16. Diffusion echo attenuation of the oil signal for a fresh $40 \mathrm{wt} \%$ toluene emulsion observed at $\Delta=40 \mathrm{~ms}$ (multiexponential decay) and $\Delta=1.3 \mathrm{~s}\left(\Delta_{\text {switch }}\right.$, single exponential decay) and for a fresh $40 \mathrm{wt} \% p$-xylene emulsion observed at $\Delta=\mathbf{4 0}$ ms (multiexponential decay) and $\Delta=1.5 \mathrm{~s}\left(\Delta_{\text {switch }}\right.$ single exponential decay).

For short observation times, $\Delta$, the echo attenuation of the oil signal decays as a multiexponential consistent with the polydispersity of the samples. For a given observation time, $\Delta$, when a multiexponential decay is measured, the attenuation data of the $p$-xylene emulsions decay more slowly than for the toluene system (see $\Delta=40 \mathrm{~ms}$, Figure 7.16). The diffusion spectra extracted from the mutiexponential attenuation data of a $40 \mathrm{wt} \% p$-xylene emulsion upon varying $\Delta$ using an inverse Laplace transform are given in Figure 7.17. 


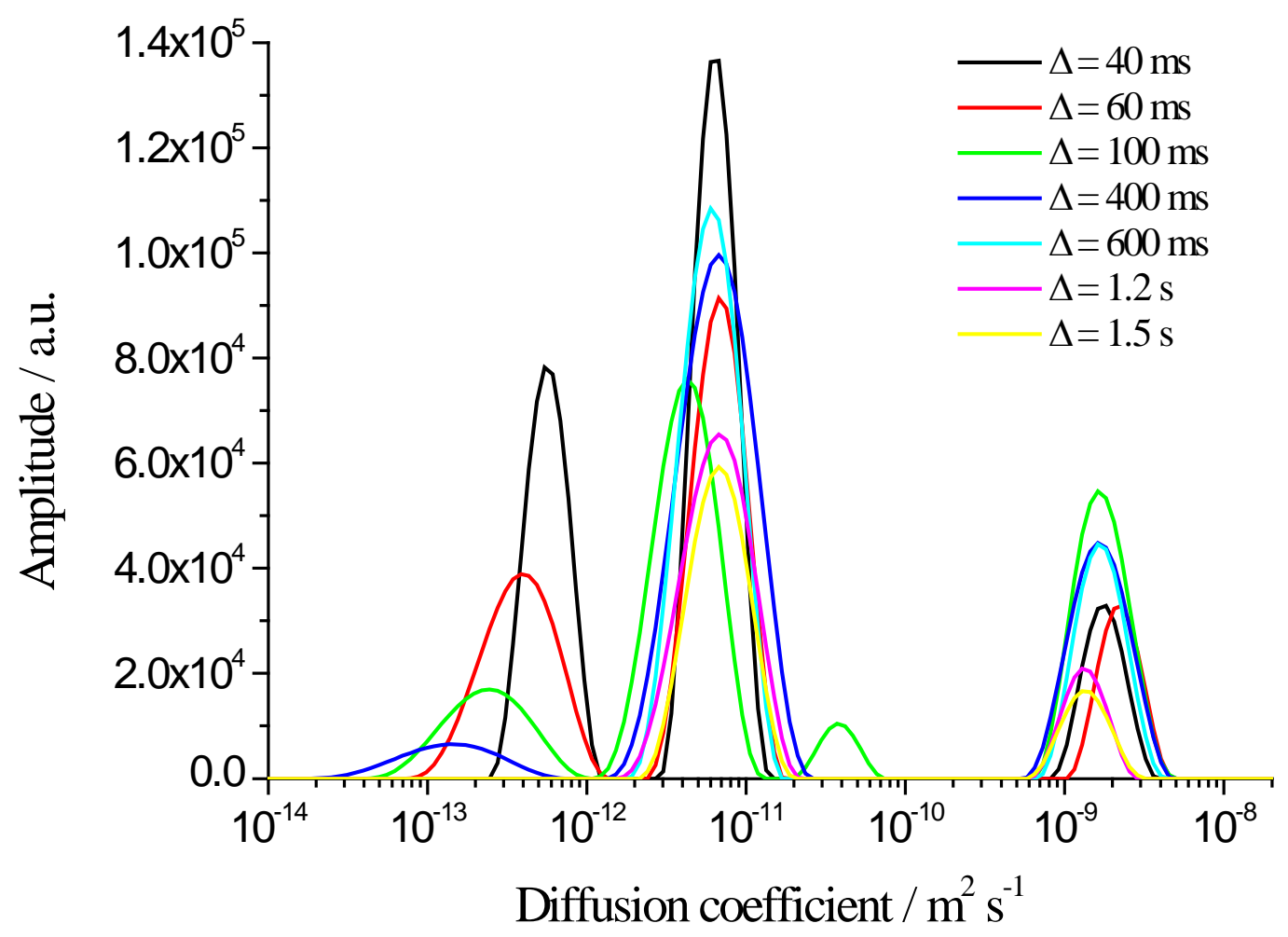

Figure 7.17. Diffusion spectra of the oil signal as a function of $\Delta$ for a fresh $40 \mathrm{wt} \% p$-xylene emulsion.

For $\Delta=40 \mathrm{~ms}$, the diffusion spectrum of the oil phase comprises three peaks centred at $\sim D=1.6 \times 10^{-9}, \sim 6.7 \times 10^{-12}$ and $\sim 5.5 \times 10^{-13} \mathrm{~m}^{2} \mathrm{~s}^{-1}$, respectively.

The peak associated with fastest diffusion is attributed, as for the toluene system, to the unrestricted diffusion of $p$-xylene molecules dissolved directly in the aqueous continuous phase.

Likewise, the peak associated with intermediate diffusion, with limits $\sim 2.5 \times 10^{-12}$ and $\sim 1.5 \times 10^{-11} \mathrm{~m}^{2} \mathrm{~s}^{-1}$, corresponds to pure unrestricted diffusion as evidenced by the mean square displacements of the oil associated with all the components of this peak $\left(<Z^{2}>=2 D \Delta\right)$ being increasing functions of $\Delta$ (Figure 7.18). Using the Stokes-Einstein equation (Eq. 3.4), this peak represents the unrestricted diffusion of droplets from $\sim 30 \mathrm{~nm}$ to $\sim 0.2 \mu \mathrm{m}$ diameter. Note that in contrast to the toluene system, this peak does not include the unrestricted diffusion of oil swollen micelles (6-50 nm diameter, Figure 7.2). A small peak centered at $\sim 3.7 \times 10^{-11} \mathrm{~m}^{2}$ $\mathrm{s}^{-1}$ appears only occasionally in the diffusion spectrum, e.g. for $\Delta=100 \mathrm{~ms}$ in Figure 7.17, representing the unrestricted diffusion of oil swollen micelles from $\sim 6$ to $\sim 20 \mathrm{~nm}$ diameter (Eq. 3.4). The occasional detection of this peak is due to 
the very small amount, volume-wise, of the oil swollen micelles solubilised in the aqueous continuous phase of $p$-xylene emulsions as compared with the toluene system.

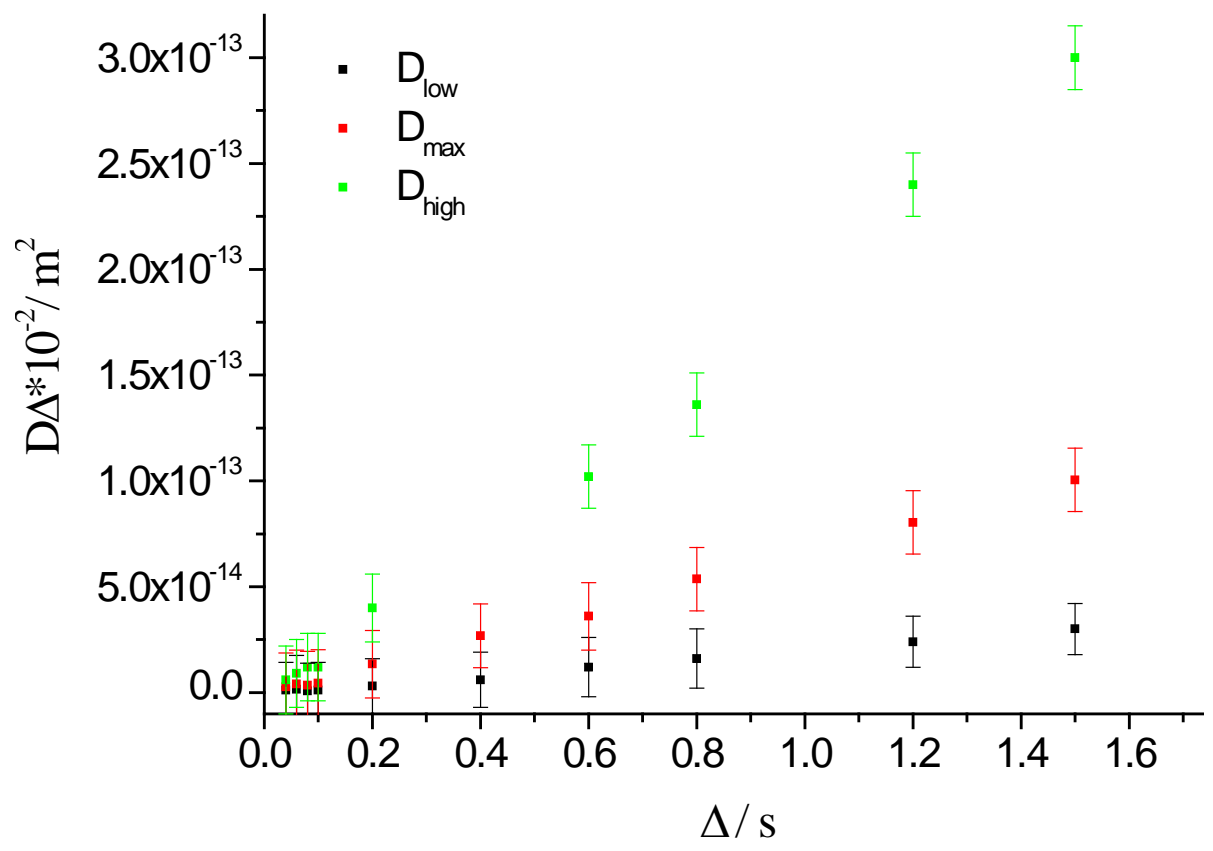

Figure 7.18. Plot of the product $D \Delta$ as a function of $\Delta$ for the low end tail $D_{\text {low }}$, maximum $D_{\max }$ and high end tail $D_{\text {high }}$ of the middle peak in the $p$-xylene diffusion spectra obtained for a fresh $40 \mathrm{wt} \%$ oil emulsion.

The peak associated with the slowest diffusion of the spectrum, centred at $\sim 5.5 \times$ $10^{-13} \mathrm{~m}^{2} \mathrm{~s}^{-1}$ for $\Delta=40 \mathrm{~ms}$, is a combination of unrestricted (fast end tail) and restricted diffusion (slow end tail) as seen in Figure 7.19. The mean square displacement of the oil associated with the fast end tail of this peak is an increasing function of $\Delta$ indicating that pure unrestricted diffusion is probed whereas the mean square displacement associated with the slow end tail is constant, i.e. the corresponding motion is restricted for $\Delta>40 \mathrm{~ms}$. This results in the mean square displacement of the oil corresponding to the maximum of this peak being an attenuated rising function of $\Delta$. For $\Delta=40 \mathrm{~ms}$, when the diffusion of the oil is unrestricted, the peak associated with slowest diffusion represents the unrestricted motion of droplets from $\sim 0.3$ to $\sim 1.7 \mu \mathrm{m}$ diameter (Eq. 3.4). Note that droplets larger than $\sim 1.7 \mu$ m represent a volume fraction which is too small to be detected by the spectrometer. For longer observation times, the diffusion of the largest droplets becomes restricted by the presence of the other droplets, hence 
the low end tail of the peak associated with slowest diffusion moves to slower diffusion coefficients. As such, the analysis of the diffusion spectrum of the oil for short values of $\Delta$ is consistent with the SLS data (Figure 7.1), i.e. there is a larger number of medium size droplets ( $\sim 0.3$ to $1.5 \mu$ m diameter) in $p$-xylene than in toluene emulsions resulting in their motions being detected in fresh samples.

Upon increasing $\Delta$, the contribution of the peak associated with slowest diffusion is increasingly reduced, as for the toluene system, and by $600 \mathrm{~ms}$ restricted diffusion is no longer detected as it now lies below the instrument detection limit. From $\Delta=600 \mathrm{~ms}$, the peak centered at $\sim 6.7 \times 10^{-12} \mathrm{~m}^{2} \mathrm{~s}^{-1}$ progressively narrows but is never a single delta function even when the echo attenuation decays as a single exponential, i.e. for $\Delta \geq 1.5$ s (Figure 7.17).

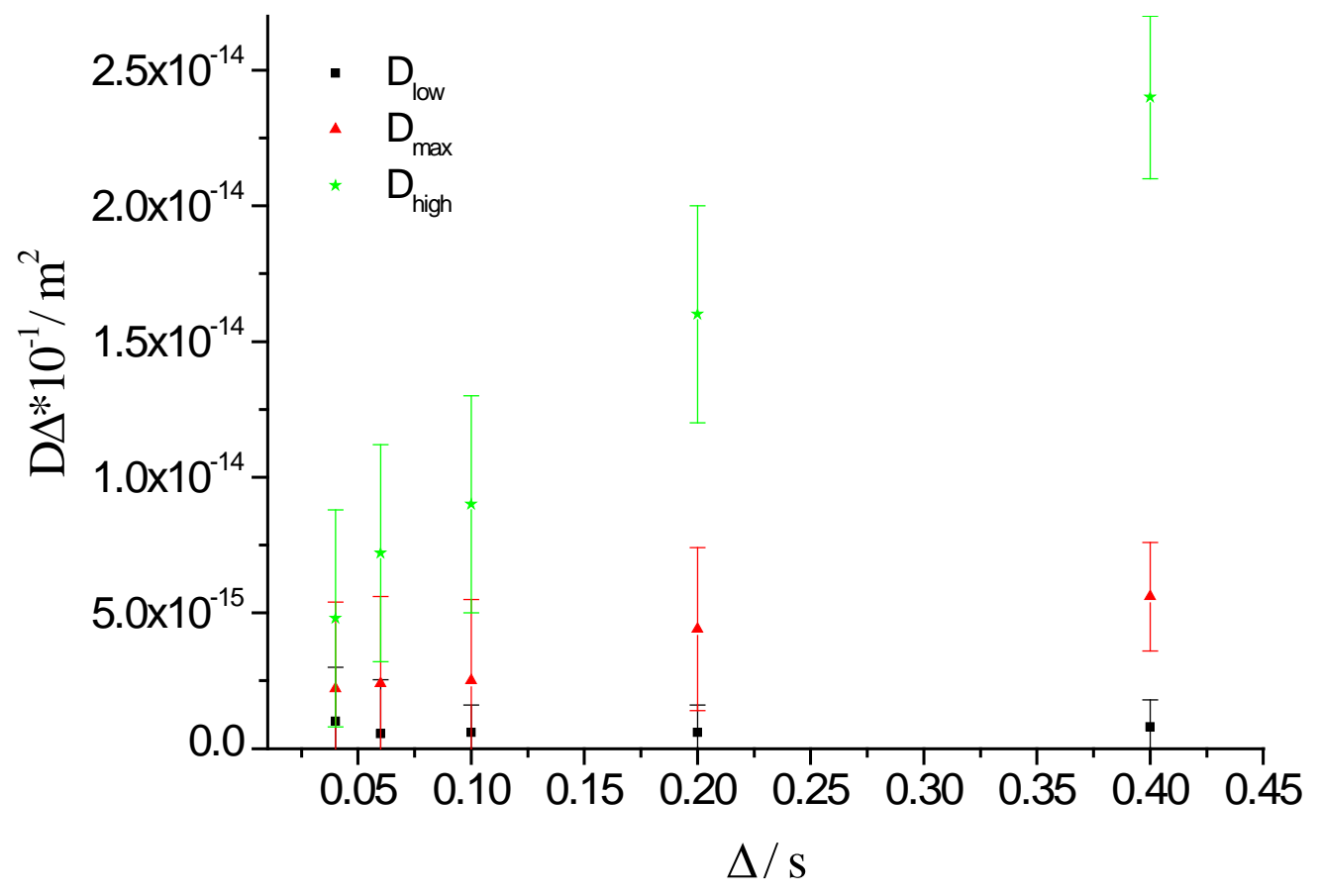

Figure 7.19. Plot of the product $D \Delta$ as a function of $\Delta$ for the low end tail $D_{\text {low }}$, maximum $D_{\text {max }}$ and high end tail $D_{\text {high }}$ of the peak associated with the slowest diffusion in the $p$-xylene diffusion spectra obtained for a fresh $40 \mathrm{wt} \%$ oil emulsions.

\subsubsection{Dynamics}

In Figure 7.20 is shown the time scale of the oil exchange, $\Delta_{\text {switch }}$, determined by PFG-NMR (switching time between a multiexponential and a single exponential decay of the echo attenuation of the oil signal as a function of gradient strength) 
upon varying oil concentration for $p$-xylene emulsions in comparison with the toluene system.

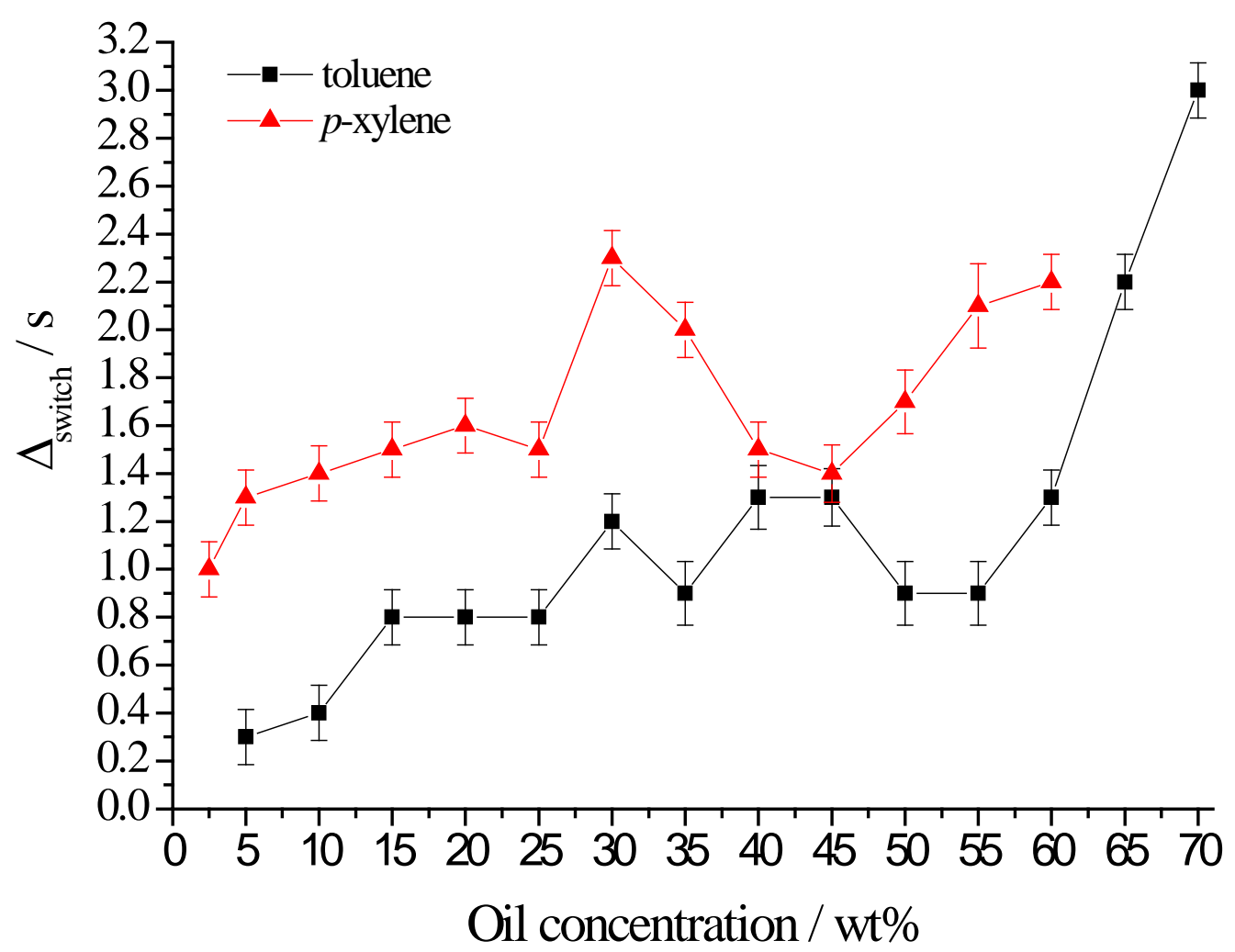

Figure 7.20. NMR switching time $\Delta_{\text {switch }}$ versus oil concentration for fresh $p$-xylene and toluene-based emulsions between 2.5 and $70 \mathrm{wt} \%$ oil.

Between 2.5 and $60 \mathrm{wt} \% p$-xylene, $\Delta_{\text {switch }}$ varies between $\sim 1$ and $\sim 2.3$ s, i.e. the time scale of the oil exchange occurring between $p$-xylene droplets is on average $800 \mathrm{~ms}$ longer than for the toluene system. This is in agreement with the mean droplet size in the $p$-xylene system being on average larger than in the toluene system as evidenced by SLS (Figure 7.1). Hence, $p$-xylene droplets move and interact with each other, on average, more slowly than toluene droplets leading to the reduction of the collision frequency.

Considering those emulsions prepared with $p$-xylene between 65 and $70 \mathrm{wt} \%$, the echo attenuation of the oil signal as a function of the gradient strength is multiexponential up to $\sim 3$ s. However, it is known, based on the confocal microscopy experiments where all droplets fluoresced yellow within $\sim 10$ min of mixing two separately labelled samples, with no change of the droplet size distribution (Figure 7.9), that oil exchange continues to occur at very high $p$ - 
xylene concentration. As such, the time constant of the oil transfer in these samples is longer than the longest NMR observation time available.

Overall, the switching time is an increasing function of $p$-xylene concentration from 2.5 to $60 \mathrm{wt} \%$ (Figure 7.20). The increase of the mean droplet size upon increasing $p$-xylene concentration (see SLS data, Figure 7.1) may partially account for the time scale of oil exchange being an increasing function of oil concentration since the droplet encounter frequency decreases.

Moreover, the four concentration regions apparent from the macroscopic phase separation data between 2.5 and $60 \mathrm{wt} \%$ p-xylene can also been distinguished in the plot of $\Delta_{\text {switch }}$ versus $p$-xylene concentration though the boundaries are slightly shifted. Based on the macroscopic phase separation data, these four concentration domains are 2.5 to $5 \mathrm{wt} \%, 10$ to $20 \mathrm{wt} \%, 25$ to $35 \mathrm{wt} \%$ and 40 to $55 \mathrm{wt} \%$ oil. The inspection of the PFG-NMR data in Figure 7.20 indicates that these appear as 2.5 to $5 \mathrm{wt} \%, 10$ to $25 \mathrm{wt} \%, 30$ to $40 \mathrm{wt} \%$ and 45 to $60 \mathrm{wt} \%$ oil. This suggests that the factors controlling the rate of oil exchange and those controlling the rate of droplet growth are similar.

Measurement of the diffusion coefficients of the oil swollen micelles diffusing freely in the expelled aqueous continuous phase of aged $p$-xylene emulsions suggests that these get larger upon increasing $p$-xylene concentration (Figure 7.21). The diffusion coefficient corresponding to the unrestricted diffusion of the p-xylene swollen micelles upon increasing oil concentration was calculated using an inverse Laplace transform based on the signal from the expelled aqueous phase of phase separated emulsions. The diffusion coefficients span from $(2.8 \pm 0.3) \times$ $10^{-11}$ to $(1.2 \pm 0.3) \times 10^{-11} \mathrm{~m}^{2} \mathrm{~s}^{-1}$ upon increasing $p$-xylene concentration from 15 to $40 \mathrm{wt} \%$ and correspond to a size range of 16 to $36 \mathrm{~nm}$ diameter (Eq. 3.4) fitting well with the size range measured by DLS (6 to $50 \mathrm{~nm}$, Figure 7.2). The diffusion coefficient of the oil swollen micelles is a decreasing function of $p$-xylene concentration between 15 and $40 \mathrm{wt} \%$ indicating that the size of the oil swollen micelles increases with oil content. 


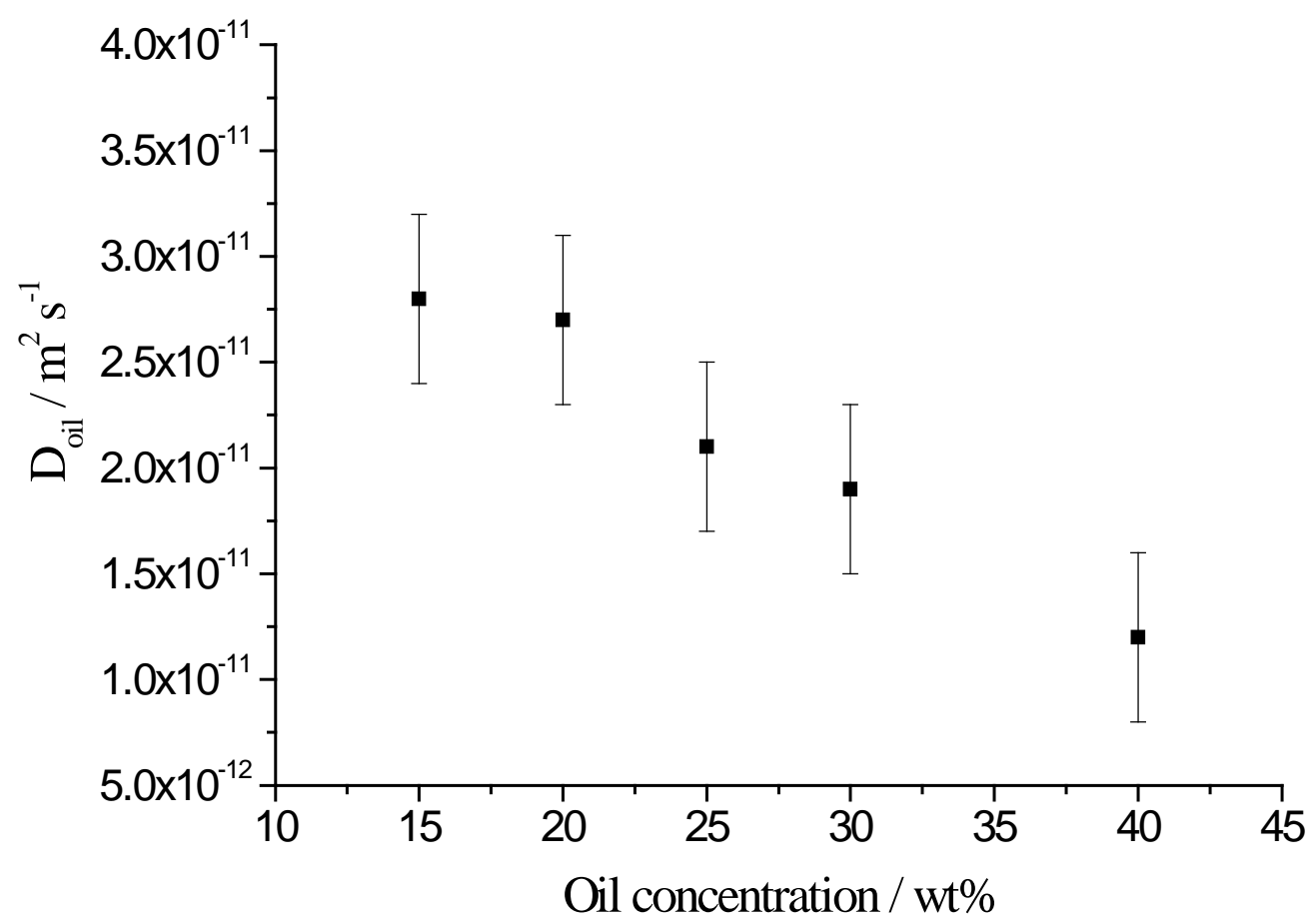

Figure 7.21. Diffusion coefficients of the oil signal detected in the expelled aqueous phase of aged emulsions prepared with $p$-xylene between 15 and $40 \mathrm{wt} \%$ for $\Delta$ between $6.3 \mathrm{~ms}$ and $3 \mathrm{~s}$.

From the echo attenuations of $40 \mathrm{wt} \%$ oil emulsions (Figure 7.16) decaying as single exponentials for $\Delta \geq \Delta_{\text {switch, }}$ i.e. $\Delta \geq 1.3 \mathrm{~s}$ and $\Delta \geq 1.5$ s for toluene and $p$ xylene, respectively, the diffusion coefficient value associated with the motional averaging (slowest slope) of the oil diffusion in the $p$-xylene system is smaller than for the toluene system. This trend is true for all $p$-xylene concentrations between 5 and 60 wt\% as seen in Figure 7.22. 


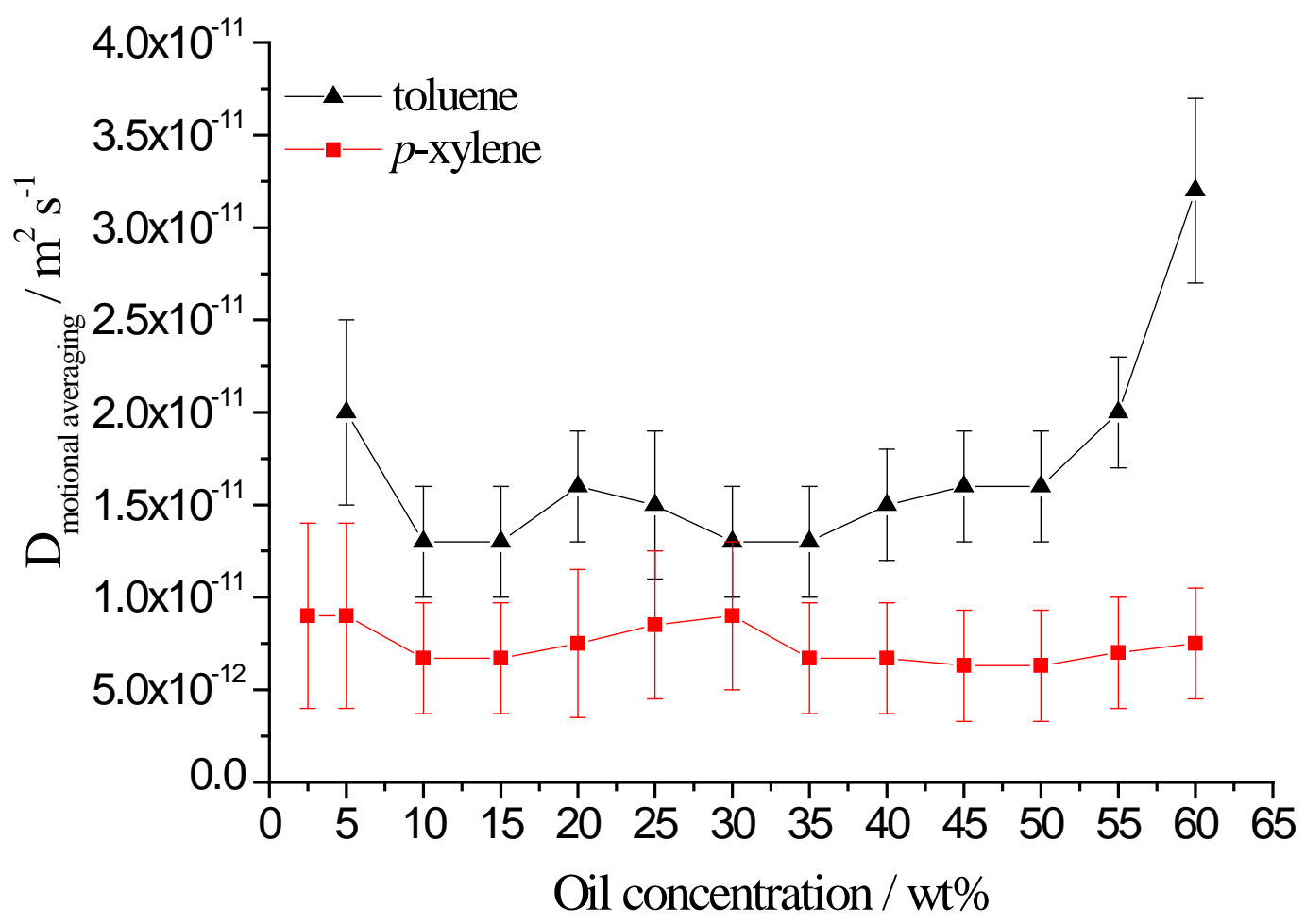

Figure 7.22. Motional averaging of the oil diffusion versus oil concentration for toluene and $p$-xylene-based emulsions.

The motional averaging of the oil diffusion in $p$-xylene emulsions is independent of oil concentration with a mean value of $\langle D\rangle=(7.5 \pm 0.3) \times 10^{-12} \mathrm{~m}^{2} \mathrm{~s}^{-1}$ as compared with $\left\langle D>=(1.5 \pm 0.2) \times 10^{-11} \mathrm{~m}^{2} \mathrm{~s}^{-1}\right.$ between 5 and $55 \mathrm{wt} \%$ oil in toluene emulsions. This quantitatively shows that $p$-xylene molecules exchange a fewer number of times over the same duration as compared with toluene molecules at equal oil concentration. This partly reflects the smaller droplet collision frequency in $p$-xylene emulsions as a result of the augmentation of the mean droplet size.

The increase of the mean droplet size with oil content in $p$-xylene emulsions (Figure 7.1) results in the decrease of the droplet encounter frequency superposed to oil molecules being able to travel a longer distance within the droplets over the same time period before exchanging. This leads to the motional averaging of the $p$-xylene diffusion, proportional to the distance travelled by the oil molecules per unit time, being constant upon increasing oil content (Figure 7.22) as the decrease of the collision frequency is countered by the increase in the mean droplet size. 


\subsection{Discussion}

From the laser scanning confocal microscopy data, oil exchange, which is not coupled to droplet growth, occurs in three different systems of oil/Triton X100/water emulsions where the oil is one of toluene, $p$-xylene or octane. In Chapter 6, it was evidenced that coalescence was the main destabilization mechanism within the first 6 hours following emulsification for toluene emulsions, independently of oil concentration, in addition to Ostwald ripening occurring within the first hour of the emulsion life for the highly concentrated samples (65 wt\% toluene).

Furthermore, octane-in-water emulsions, stabilized by Triton X-100, destabilize via an enhanced Ostwald ripening mechanism within the first 6 hours following emulsification, independently of oil concentration [49]. Therefore, oil exchange is observed in three oil systems that destabilize via different mechanisms. This strongly confirms that such an oil transfer does not pertain to emulsion destabilization but rather occurs as a separate process.

From the determination of the switching time, $\Delta_{\text {switch, }}$ using PFG-NMR, for toluene and $p$-xylene emulsions prepared with oil concentrations between 2.5 and $70 \mathrm{wt} \%$, it is evident that the time scale of the oil exchange increases from toluene to $p$-xylene by $\sim 800 \mathrm{~ms}$ (Figure 7.20 ). This is consistent with the mean droplet size of the $p$-xylene emulsions being on average larger than the toluene emulsions as demonstrated by the SLS data (Figure 7.1), resulting in the decrease of the droplet encounter frequency at equal oil concentration since droplets move on average more slowly. As such, a slower rate of oil exchange is observed which is also in agreement with the smaller value of the motional averaging of the oil diffusion in the $p$-xylene system measured by PFG-NMR for concentrations between 5 and $60 \mathrm{wt} \%$ p-xylene (Figure 7.22).

Similarly, O/W emulsions in the octane/Triton X-100/water system, prepared according to the same emulsification method as for the toluene and p-xylene systems (see section 3.3), were investigated using SLS and PFG-NMR [133]. Octane emulsions could be prepared only between 40 and $60 \mathrm{wt} \%$ oil. Octane droplets are on average larger as compared with the toluene and $p$-xylene systems. From 40 to $50 \mathrm{wt} \%$ octane, droplets are comprised between $\sim 0.3$ and $\sim 3.5 \mu \mathrm{m}$ 
diameter with a maximum at $\sim 1.2 \mu \mathrm{m}$. For $55 \mathrm{wt} \%$ octane, the maximum shifts to $\sim 1.5 \mu \mathrm{m}$ with droplets as large as $5 \mu \mathrm{m}$ being formed. For $60 \mathrm{wt} \%$ octane, the maximum shifts to $1.8 \mu \mathrm{m}$ with droplets as large as $\sim 8 \mu \mathrm{m}$ being present. Additionally, PFG-NMR experiments show that no motional averaging of the octane diffusion is detected for times up to $\sim 2 \mathrm{~s}$ [133]. Since oil exchange does occur in octane emulsions, as evidenced by our confocal microscopy data for a 40 wt\% octane sample (Figure 7.10), the time constant for the oil transfer is therefore longer than $\sim 2 \mathrm{~s}$. The cryo-SEM micrographs of octane emulsions (Figure 7.14 and Figure 7.15) also indicate, at least qualitatively, that the time scale of the oil exchange process is the longest of the three oil systems since no direct droplet contact is observed within the $\sim 1 \mathrm{~s}$ of the experimental time scale.

The increase of the mean droplet size observed in oil/Triton X-100/water emulsions in the order of toluene to $p$-xylene to octane contributes to the decrease of the rate of oil exchange in the same order as a result of the decrease of the droplet collision frequency. However droplet size cannot be the sole contributor to the decrease of the rate of oil exchange. From the data reported in chapter 6 it was evidenced that the properties of the interfacial domain, including the surfactant packing at the oil/water interface, are of fundamental importance in controlling the time scale of the oil exchange. In an oil transfer occurring either via oil permeation upon droplet collision or reversible coalescence of the droplets, the time scale of such an exchange process is expected to also depend on the mutual interactions between the oil and the Triton X-100 molecules.

The macroscopic phase separation data of the $p$-xylene emulsions indicate that the rate of phase separation resulting from droplet growth is slower than that of the toluene emulsions as seen by the times to reach complete phase separation. Toluene emulsions phase separate between $\sim 186$ to $\sim 480$ h (Table 6.1 to Table 6.3) whereas it takes $\sim 337$ to $\sim 800 \mathrm{~h}$ for the $p$-xylene emulsions (Table 7.2) between 5 and $60 \mathrm{wt} \%$ oil. Coalescence is assumed to be the main destabilization mechanism of the $p$-xylene emulsions, as for the toluene emulsions, based on the strong similarities between these two oil systems with respect to macroscopic phase separation (most of the aqueous continuous phase is expelled within a short period of time of a few hours, Figure 6.1 and Figure 7.3) and evolution of the droplet size distribution with time (Figure 6.8 and Figure 7.23 ). Hence, the 
respective rates of phase separation of the toluene and $p$-xylene systems are not consistent with the normal situation where the rate of droplet growth via a coalescence mechanism is proportional to the initial mean droplet size [121]. Therefore, the Triton X-100 layer covering $p$-xylene droplets must be more rigid with respect to oil transfer leading to emulsion destabilisation as compared with the layer covering toluene droplets.

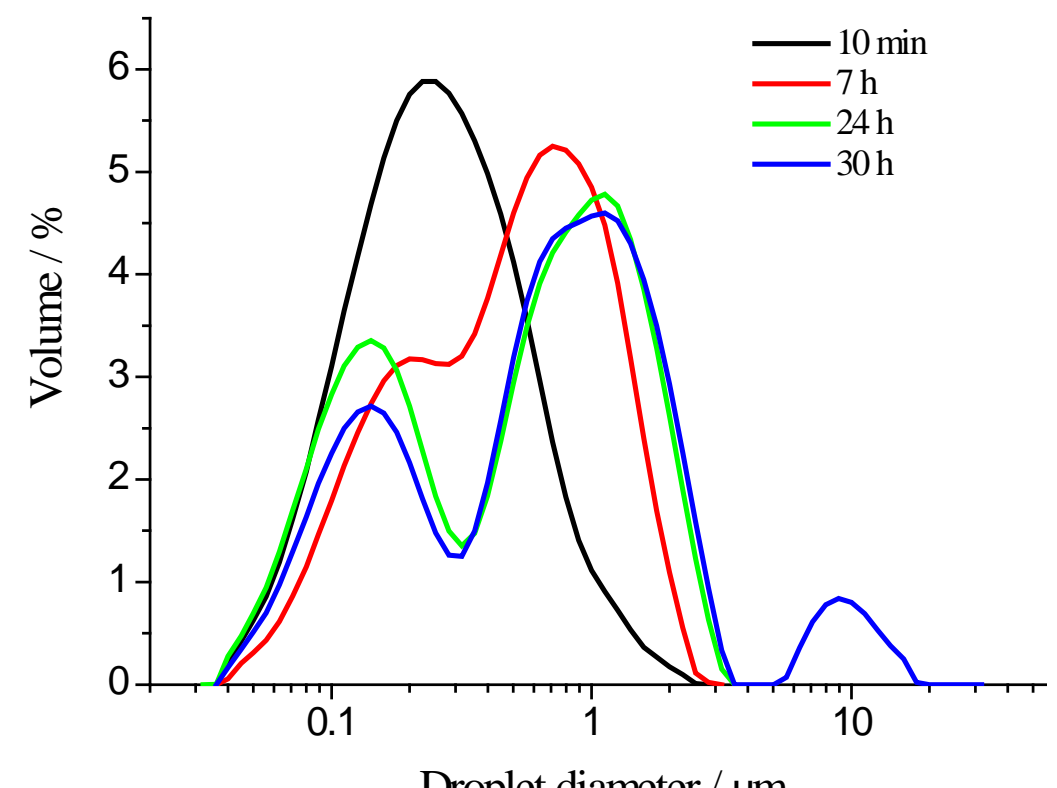

Figure 7.23. Droplet size distributions of a $50 \mathrm{wt} \%$-xylene emulsion as a function of time using SLS.

In an enhanced Ostwald ripening mechanism, the rate of droplet growth is mainly controlled by the interactions between the oil and the surfactant molecules upon oil transfer occurring between oil droplets and oil swollen micelles [49, 84]. Considering the macroscopic phase separation behaviour of the octane/Triton X100/water emulsions, destabilizing via enhanced Ostwald ripening, the rates of phase separation, resulting from droplet growth, are slower than those of the toluene and p-xylene systems. Indeed, the time to reach complete phase separation in octane emulsions is $\sim 600 \mathrm{~h}$, independently of oil concentration (Figure 7.24), as compared with $\sim 550 \mathrm{~h}$ in the $p$-xylene system from 40 to $55 \mathrm{wt} \%$ p-xylene (Table 7.2) and $\sim 383$ to $\sim 480 \mathrm{~h}$ in the toluene system from 40 to $60 \mathrm{wt} \%$ toluene (Table 6.1). Based on these data, the Triton X-100 membrane is more robust, with respect to oil transfer leading to emulsion destabilization, in the order 
of toluene to p-xylene to octane. The same analysis can be applied to the oil transfer occurring via oil permeation or reversible coalescence since it was observed experimentally that the parameters affecting the rate of droplet growth, e.g. oil content, are similar to those affecting the rate of oil exchange. The mechanisms of oil permeation, reversible coalescence, irreversible coalescence and enhanced Ostwald ripening all involve the formation of transient holes in the emulsifier thin film, the kinetics of which may control the rate of oil transfer leading or not to droplet growth.

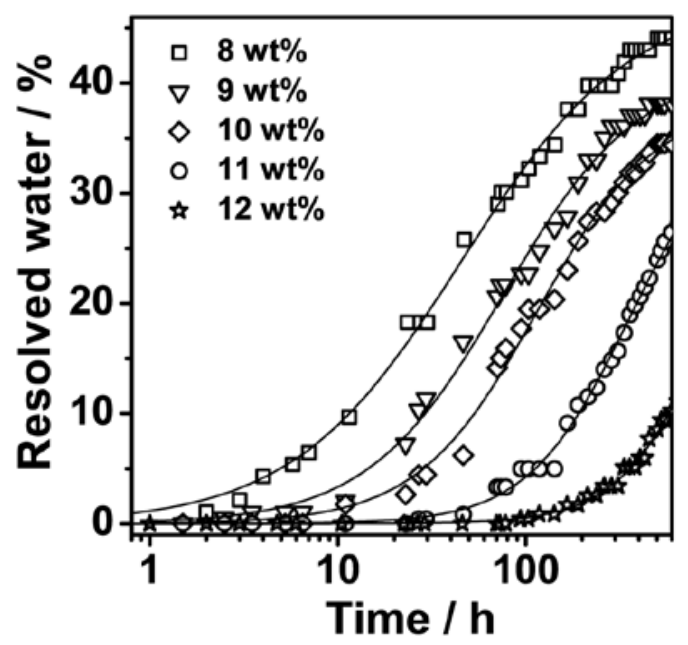

Figure 7.24. Amount of expelled aqueous phase as a function of time taken from reference [49], for octane/Triton X-100/water emulsions prepared with Triton X-100 between 8 and 12 wt $\%$ while maintaining the octane-to-Triton $X-100$ weight ratio to 5:1.

Kabalnov and Wennerström [78] argue that the surfactant spontaneous curvature affects both the self-assembly of the surfactant molecules in the phase diagram as well as the stability of emulsions. The energy cost for hole nucleation in emulsion systems, where the surfactant curvature is not at equilibrium, depends on the surfactant equilibrium curvature. Schmitt et al. [90] suggest that oils with the longest hydrophobic chains hardly penetrate into the surfactant layer, hence maintaining a highly positive spontaneous mean curvature of the surfactant layer in $\mathrm{O} / \mathrm{W}$ emulsions. The interfacial membrane is then well stabilized against hole nucleation (step required for oil permeation or reversible coalescence) due to the large energy barrier for the formation of holes since it requires the surfactant layer to adopt a local negative mean curvature around the hole [90, 102]. On the contrary, short oil chains are able to swell the surfactant layer and increase the distance between the surfactant tails while maintaining the same distance between 
the surfactant head groups, hence imposing a less positive spontaneous curvature in $\mathrm{O} / \mathrm{W}$ emulsions and reducing the activation energy for hole nucleation and oil transfer [78]. For example, Schmitt et al. experimentally verified that the rate of coalescence, limited by the rate of hole nucleation, is a decreasing function of the hydrocarbon chain length from heptane through to dodecane in concentrated alkane-in-water emulsions stabilized by a mixture of $\mathrm{C}_{12} \mathrm{E}_{5}$ and $\mathrm{C}_{10} \mathrm{E}_{5}$ [134]. Likewise, Schmitt et al. determined that the rate of permeation-driven Ostwald ripening, where oil transfer occurs upon collision via the transposition of two transient holes, is also a decreasing function of the alkane chain length in $\mathrm{O} / \mathrm{W}$ emulsions [90].

Several studies have focused on the effect of oil/emulsifier/water interactions on the rate of emulsion destabilization via Ostwald ripening [116, 117, 135]. The Lifshitz-Slyozov-Wagner model [116, 117] predicts that the rate of standard Ostwald ripening is an increasing function of the oil/water interfacial tension in addition to the oil molar volume, the diffusion coefficient and the solubility of the oil in the aqueous continuous phase. Weiss and coworkers experimentally measured faster ripening rates in the sterically stabilized $\mathrm{O} / \mathrm{W}$ emulsions with the highest interfacial tensions [135]. Furthermore, the kinetics of solubilisation of oil molecules from emulsion droplets into oil swollen micelles upon enhanced Ostwald ripening have been largely investigated [118, 136-138]. Three solubilisation mechanisms have been proposed including the direct solubilisation of oil molecules in the aqueous phase followed by the incorporation into micelles, the fusion/fission of an oil droplet with a micelle and the spontaneous "buddingoff" of oil and surfactant molecules from a droplet to form a micelle [139, 140]. Weiss and coworkers [84] found that the solubilisation rate and maximum solubilising capacity of the micelles is a decreasing function of the hydrocarbon chain length and is larger for unsaturated than for saturated hydrocarbons of the same chain length in alkane-in-water emulsions stabilized by a nonionic surfactant using light scattering techniques and turbidity experiments. Considering either a fusion/fission or a "budding-off" mechanism for oils with low water solubilities, oil molecules confined within the hydrophobic interior of the droplets must cross the more polar environment associated with the surfactant headgroups before being incorporated into the micelles. As such, they suggested that the rate of oil transfer is increasingly facilitated upon decreasing the 
hydrocarbon chain length or increasing the chain polarity due to unsaturation because of the concomitant decrease of the energy barrier associated with the exposure of nonpolar molecules to a more polar environment. Additionally, upon increasing the alkane chain length there is a decrease of the configurational entropy associated with the restricted volume available to larger hydrocarbons within the micelles and the concomitant increase of the protrusion of the chains into the polar environment of the surfactant layer, which is energetically unfavorable.

Toluene molecules are able to readily swell the Triton X-100 layer due to the stabilizing interactions between their respective aromatic rings. From toluene to octane in oil/Triton X-100/water emulsions, the chemical affinity for Triton X100 decreases because of the loss of the stabilizing interactions between the aromatic rings. This is experimentally verified by the higher solubility of Triton $\mathrm{X}$-100 in toluene than in octane. Moreover, using $p$-xylene instead of toluene increases the effective volume of the oil and reduces its ability to penetrate the surfactant brush. As such, from toluene to $p$-xylene to octane the ability of the oil to penetrate the Triton X-100 layer decreases. This leads to the concomitant decrease of the distance between the Triton X-100 chains while maintaining the same distance between the head groups, resulting in a more positive spontaneous curvature of the Triton X-100 layer and the increase of the activation energy for hole nucleation in the order of toluene to $p$-xylene to octane. This corroborates the observed slower rates of oil exchange in the same order.

Considering the octane emulsions stabilized by Triton X-100, only the emulsions prepared with oil between 40 and $60 \mathrm{wt} \%$ could be formed, i.e. three of the four microstructures obtained with toluene upon varying oil content (low-oil content, bicontinuous and closed-cell foam) could not be realised and the minimum energy input required to form stable emulsions was larger [133]. The reduction of the number of microstructures obtained using octane instead of toluene is consistent with the oil/water interfacial tension being augmented from toluene $(\gamma=36.1$ $\mathrm{mN} / \mathrm{m}, 298 \mathrm{~K},[141])$ to n-octane $(\gamma=51.29 \mathrm{mN} / \mathrm{m}, 298 \mathrm{~K},[142])$. As such, the interface can be more easily manipulated in toluene emulsions including strongly positive mean curvature (low-oil content region), zero mean curvature at low energy input (bicontinuous), through to less positive curvature (high-oil content 
region). Systems with very low interfacial tensions such as microemulsions are able to manipulate the surfactant mean curvature from highly positive through to highly negative coupled to a zero curvature bicontinous microemulsion in between simply by a small change in composition [143]. Because of the high octane/water interfacial tension, it would be energetically unfavorable for the octane system to form a low-surfactant packing microstructure at low oil content as for the toluene system.

However, the increase of the oil/water interfacial tension from toluene to $p$-xylene $(\gamma=37.77 \mathrm{mN} / \mathrm{m}, 298 \mathrm{~K},[144])$ alone does not account for the observed augmentation of the number of microstructures from four to five based on the macroscopic phase separation data. This may be due to the $p$-xylene system having a larger Gibbs elasticity, defined as the capacity of films to increase their tension upon stretching and indicative of the film stability [145].

The diffusion spectra extracted from the multiexponential decay attenuations, associated with the $p$-xylene emulsions upon varying the NMR observation time, indicate that the volume-weighted signal of the oil swollen micelles is not always apparent in the spectra for the same emulsion sample, as opposed to the toluene emulsions. As such, the number of oil swollen micelles in the aqueous continuous phase most likely decreases upon using $p$-xylene instead of toluene, which is consistent with more surfactant being situated at the droplet interface for all oil concentrations in the $p$-xylene emulsions. Oil swollen micelles can be formed in the continuous phase of emulsions if there is an excess of surfactant molecules once the surface of the droplets is covered. The driving force for the formation of oil swollen micelles is the tendency of the surfactant layer to adopt its equilibrium mean curvature [84]. The decrease of the number of oil swollen micelles in the $p$ xylene system as compared with the toluene system, despite the same oil-tosurfactant weight ratio and a larger interfacial area, suggests that the surfactant packing at the surface of the $p$-xylene droplets is more important. This also corroborates the observed slower rates of both oil exchange and macroscopic phase separation as there is an increase in the steric repulsive forces between the oil droplets from toluene to $p$-xylene.

The three oil/Triton X-100/water emulsion systems where the oil is toluene, $p$ xylene or octane have vastly different properties with respect to the Triton X-100 
layer and mutual oil/Triton X-100/water interactions, as evidenced by the nature of the destabilization mechanisms, the diversity of the microstructures realized, the surfactant packing at the surface of the oil droplets and the Triton X-100 equilibrium mean curvature. Despite these striking differences, oil exchange via oil permeation upon collision or reversible coalescence of the droplets does occur in these three oil systems, suggesting the universality of the oil exchange process in kinetically stabilized emulsion systems. This is an important contribution to the general knowledge associated with the dynamics of colloidal systems.

\subsection{Conclusion}

Oil exchange between emulsion droplets via oil permeation or reversible coalescence was observed by confocal microscopy in oil/Triton X-100/water emulsions where the oil is one of toluene, $p$-xylene or octane. The characteristics of the Triton X-100 layer in these three oil systems are very different in terms of molecular packing and spontaneous mean curvature which are controlled by the oil/Triton X-100/water mutual interactions. Therefore, these three systems have different droplet size distributions, microstructures and mechanisms of instability including coalescence and enhanced Ostwald ripening. However, they all undergo oil exchange, suggesting the universality of such a process in kinetically stabilized emulsion systems.

While the increase of the mean droplet size contributes to the increase of the time scale of oil exchange, $\Delta_{\text {switch }}$, from toluene to $p$-xylene to octane as measured by PFG-NMR, the macroscopic phase separation data reveal that the Triton X-100 surfactant layer is more robust with respect to oil transfer in the same order. This may be explained in terms of the spontaneous mean curvature of the surfactant layer becoming more positive from toluene to $p$-xylene to octane due to the decrease of the oil penetration into the surfactant brush, hence increasing the energy barrier for hole nucleation required in the oil exchange process. Additionally, evidence is provided for the surfactant coverage being denser at the surface of $p$-xylene droplets as compared with toluene droplets based on the reduction of the number of oil swollen micelles resulting in oil transfer being further hindered. 


\section{Chapter 8 General conclusion and future work}

\subsection{General conclusion}

The O/W emulsion system oil/Triton X-100/water was investigated, where the oil is one of toluene, $p$-xylene or octane. Triton X-100 is a nonionic surfactant. Through this study, a better understanding of the correlations between the microstructure and the dynamics of this ternary system was gained upon varying oil concentration, droplet size, emulsion age, oil type and ionic strength while maintaining the oil-to-surfactant weight ratio, temperature, energy input and emulsification method constant.

The occurrence of an oil exchange between oil droplets, for three different oil types, that has not been reported previously in kinetically stabilized emulsion systems, is evidenced. Such an oil transfer occurs independently of emulsion destabilization and does not lead to droplet growth.

Confocal microscopy data for toluene, $p$-xylene and octane emulsions indicate that oil droplets emit yellow fluorescence within $\sim 10$ min of mixing two separately prepared emulsions, labelled with a red and a green-fluorescing dye, respectively, with no concomitant variation of the droplet size. This yellow fluorescence is due to the solubilisation of the two water-insoluble dyes within the same droplets via oil exchange.

Furthermore, PFG-NMR experiments performed on toluene and p-xylene emulsions at long observation times, $\Delta$, demonstrate that an oil transfer between the droplets occurs faster than the experimental time scale. A motional averaging of the oil diffusion was measured, i.e. the echo attenuation of the oil signal decays as a single exponential as a function of the gradient strength. The motions of the individual droplets and oil molecules are described by a unique diffusion coefficient (motional averaging value) as if all oil molecules experience the same environment, belying the system polydispersity.

Moreover, cryo-SEM micrographs reveal an extensive droplet contact within the $\sim 1$ s of the experimental time scale (freezing time) for both the toluene and the $p$ - 
xylene systems. Superposing the data collected from the three different techniques, two possible mechanisms for such an oil exchange are hypothesized. The first one is the permeation of oil molecules upon droplet collision through transient holes in the surfactant film. The second is the reversible coalescence or partial coalescence of the droplets involving the fusion/fission of the interfacial membrane.

The time scale of the oil exchange in toluene and $p$-xylene emulsions was determined using PFG-NMR. Upon decreasing the NMR observation time, $\Delta$, a switch between a single exponential decay and a multiexponential decay, mirroring the emulsion droplet size distribution, as a function of the gradient strength, occurred at time, $\Delta_{\text {switch }}$, defined as the time constant of the oil transfer. This time constant depends on the emulsion microstructure, e.g. the droplet size distribution, the surfactant packing at the oil/water interface and the surfactant equilibrium mean curvature. In particular, the properties of the Triton X-100 layer can be manipulated through the variation of oil type, oil concentration or ionic strength, resulting in the modification of the rate of oil exchange.

The microstructure of toluene emulsions upon increasing oil content was investigated. Based on SLS data, the droplet size distribution remains unchanged from 5 to $\sim 55 \mathrm{wt} \%$ toluene, including droplets between $\sim 35 \mathrm{~nm}$ to $\sim 3 \mu \mathrm{m}$ diameter. By contrast, the volume-mean droplet size increases by $\sim 60 \%$ for toluene concentrations larger than $\sim 60 \mathrm{wt} \%$. Moreover, the presence of oil swollen micelles (9-30 nm diameter) in the aqueous continuous phase, due to an excess of surfactant molecules, was detected by DLS and PFG-NMR.

Additionally, four concentration domains, associated with distinct microstructures, have been previously identified by Liu and coworkers [40] including a low-oil content region between 5 and 20 wt\% oil, a high-oil content region between $\sim 40$ and $\sim 55 \mathrm{wt} \%$ oil, a transition region in between from $\sim 25$ to $\sim 35 \mathrm{wt} \%$ oil and a closed-cell foam region above $\sim 60 \mathrm{wt} \%$ oil.

In toluene emulsions, the time scale of the oil exchange, $\Delta_{\text {switch, }}$ is an increasing function of oil concentration from $\sim 300 \mathrm{~ms}$ to $\sim 3 \mathrm{~s}$ between 5 and $70 \mathrm{wt} \%$ oil, as evidenced by PFG-NMR. From SLS data the droplet size distribution of the toluene emulsions remains unchanged between 5 and 55 wt\% oil. Therefore, increasing toluene concentration from 5 to $55 \mathrm{wt} \%$ leads to the increase of the 
droplet concentration and the droplet collision frequency. These data lead to the expectation that the time scale of the oil exchange would be a decreasing function of the droplet encounter frequency in a system where the oil-to-surfactant weight ratio is maintained. Since this is not the case, the enhancement of the Triton X100 packing at the surface of the toluene droplets upon increasing oil concentration must account for $\Delta_{\text {switch }}$ being an increasing function of toluene concentration via the reinforcement of the steric repulsion between the oil droplets. The increase of the surfactant packing with oil content also corroborates the motional averaging value of the oil diffusion, proportional to the mean distance travelled by the oil molecules during exchange, being independent of toluene concentration between 5 and 55 wt\%.

In addition to the PFG-NMR data, SLS data of aged emulsions indicate that coalescence is the main destabilization mechanism for the toluene emulsions within the first 6 hours following emulsification, independently of oil concentration. In a system maintaining a constant surfactant packing, the rate of coalescence is expected to be proportional to both the droplet collision frequency and the initial mean droplet size. However, the rate of coalescence in toluene emulsions is a decreasing function of both the droplet concentration (from $0.86 \pm$ $0.03 \mu \mathrm{m}^{-2} \mathrm{~h}^{-1}$ for a $15 \mathrm{wt} \%$ toluene sample to $0.58 \pm 0.02 \mu \mathrm{m}^{-2} \mathrm{~h}^{-1}$ for a $40 \mathrm{wt} \%$ toluene sample) and the initial mean droplet size $\left(0.18 \pm 0.01 \mu \mathrm{m}^{-2} \mathrm{~h}^{-1}\right.$ for a 65 wt $\%$ toluene sample). This quantitatively shows that the Triton $\mathrm{X}-100$ layer is increasingly robust with respect to oil transfer via coalescence upon increasing toluene concentration, supporting the statement of the enhancement of the surfactant packing. The same trend is observed with the rates of macroscopic phase separation.

The measurement of the diffusion coefficients associated with the unrestricted diffusion of the oil swollen micelles in the expelled aqueous continuous phase of phase separated toluene emulsions indicates that the spontaneous mean curvature of the Triton X-100 layer becomes less positive upon increasing toluene concentration as the oil swollen micelles get larger. This is caused by the dehydration of the Triton X-100 head groups upon decreasing the water volume fraction. These dehydration effects facilitate oil exchange by decreasing the energy barrier for the formation of transient holes (local negative curvatures). 
However, a decrease of the rate of oil exchange is observed upon increasing toluene content and decreasing the mean interdroplet spacing because the system is kinetically stabilized by the enhancement of the Triton X-100 packing at the oil/water interface, providing a stronger steric repulsive barrier between the oil droplets.

The addition of $\mathrm{NaCl}$ in the aqueous continuous phase of toluene emulsions prior to emulsification leads to the decrease of the rate of oil exchange. In the same way to that is observed upon increasing toluene concentration, the presence of $\mathrm{Na}^{+}$and $\mathrm{Cl}^{-}$ions results in the dehydration of the Triton X-100 head groups, hence a less positive spontaneous curvature. However, these dehydration effects, facilitating oil exchange, are countered by the strong binding of the $\mathrm{Na}^{+}$ions to the surfactant layer, increasing the steric repulsive forces between the oil droplets. The effect of the nature of the oil, from toluene to $p$-xylene to octane, on the microstructure and the rate of oil exchange of the oil/Triton X-100/water emulsions was also investigated. Considering first the microstructure of the $p$ xylene emulsions as compared with the toluene emulsions, the addition of one methyl group resulted in one additional concentration domain at low oil concentration between 2.5 and 5 wt\% p-xylene as seen from the macroscopic phase separation data. With regard to the droplet size distribution, the volumebased mean droplet size is an increasing function of $p$-xylene concentration from 2.5 to $70 \mathrm{wt} \%$ although droplets are still in the range of $\sim 35 \mathrm{~nm}$ to $\sim 3 \mu \mathrm{m}$ diameter. Furthermore, the time scale of the oil exchange, $\Delta_{\text {switch, }}$ increases by $\sim 800 \mathrm{~ms}$ as compared with the toluene system, spanning from $\sim 1$ to $\sim 2.3 \mathrm{~s}$ between 2.5 and $60 \mathrm{wt} \%$ p-xylene.

Octane emulsions can only be formed between 40 and $60 \mathrm{wt} \%$ oil. Octane droplets are, on average, larger as compared with both the toluene and the $p$ xylene emulsions. Regarding the dynamics of the system, PFG-NMR experiments performed by Liu and coworkers [133] indicate that the time scale of the oil exchange must be longer than $\sim 2 \mathrm{~s}$ since no motional averaging of the oil diffusion is detected over this time frame despite oil exchange occurring, as evidenced from the confocal microscopy data.

The rate of oil exchange is inversely proportional to droplet size due to the concomitant decrease of the droplet collision frequency, as evidenced by $\Delta_{\text {switch }}$ 
being an increasing function of the mean droplet size. Although the increase of the mean droplet size from toluene to $p$-xylene to octane emulsions contributes to the decrease of the rate of oil exchange in the same order at equal oil concentration, this cannot be the sole contributor to this behaviour. The specific interactions between the oil and the Triton X-100 molecules also control the time scale of the oil exchange. The respective macroscopic stabilities of these three oil systems, destabilizing either via coalescence or enhanced Ostwald ripening, indicate that the Triton X-100 layer is increasingly robust with respect to oil transfer. Hence, the effects of oil penetration into the surfactant brush must be considered. The increase of the oil molecular size, from toluene to $p$-xylene, as well as the loss of the stabilizing interactions between the oil and the surfactant respective aromatic rings, from toluene or $p$-xylene to octane, results in the decrease of the oil penetration into the Triton X-100 layer. Therefore, the surfactant spontaneous curvature becomes more positive and the energy cost for the formation of holes increases from toluene to $p$-xylene to octane, leading to the decrease of the rate of oil exchange in the same order.

Oil exchange between emulsion droplets occurs via an oil permeation or a reversible coalescence mechanism in three oil systems that have vastly different microstructures, including droplet size distribution, surfactant packing at the oil/water interface and surfactant equilibrium mean curvature, as well as different mutual interactions between oil, Triton X-100 and water molecules. This leads to different destabilization mechanisms and diversities of microstructures upon varying oil content. Despite the properties of these three oil systems being significantly different, they all undergo oil exchange, suggesting the universality of such a process in kinetically stabilized emulsion systems. This is a significant breakthrough to the general knowledge of emulsion dynamics since these can no longer be solely depicted and driven by the balance between attractive forces (van der Waals forces) and electrostatic repulsive forces, as first suggested by the DLVO theory. Kinetic forces including all the repulsive forces stabilising the system against droplet growth (e.g. hydration forces, thermal fluctuations etc) must be incorporated into the general theoretical description of emulsion dynamics. These kinetic forces must drive the observed oil exchange process occurring with no concomittant droplet growth. Furthermore, evidence for such an oil exchange occurring between oil droplets has significant consequences with 
respect to industrial applications using emulsions as carriers of hydrophobic materials. The understanding of such an oil exchange and how this process affects the physicochemical properties of emulsion systems is crucial in order to control and manipulate the distribution of hydrophobic substances, e.g. drugs, fragrances etc, within the dispersed oil phase.

\subsection{Future work}

One of the possible future challenges is finding an oil/Triton X-100/water system with much slower dynamics of oil exchange in order to distinguish which of oil permeation or reversible coalescence is the dominant mechanism for oil transfer. For example, a system for which the oil exchange would occur on a time scale longer than $\sim 10$ minutes may enable us to visualise the membrane fusion between a red-fluorescing and a green-fluorescing oil droplet under the confocal microscope though it might still be difficult to distinguish a droplet encounter pair, for which permeation can occur, from two partially coalesced droplets.

There are different ways of decreasing the rate of oil exchange as compared with toluene, $p$-xylene and octane emulsions. For example, the use of aromatic oils with longer lateral alkyl chains, e.g. tert-butylbenzene, or longer straight hydrocarbon oils, e.g. hexadecane, may result in the decrease of the rate of oil exchange by several orders of magnitude. This would be achieved by decreasing the penetration of the oil into the Triton X-100 layer in order to impose a more positive surfactant equilibrium mean curvature, hence increase the energy cost for the formation of local negative curvatures to form a hole.

An alternative method to decrease the rate of oil exchange without changing the oil type would be to probe the oil exchange at lower temperatures. The effect of temperature on the time scale of permeation-controlled Ostwald ripening in $\mathrm{O} / \mathrm{W}$ emulsions has already been observed by Taisne and Cabane [89]. This time scale increased from $2 \mathrm{~min}$ at $26^{\circ} \mathrm{C}$ to $20 \mathrm{~min}$ at $20^{\circ} \mathrm{C}$ to $3 \mathrm{~h}$ at $14^{\circ} \mathrm{C}$. A lower temperature leads to a higher activation energy for the formation of holes at the surface of the emulsifier film, hence a decrease in the rate of oil transfer.

In order to gain further understanding about the enhancement of the surfactant packing upon increasing oil content in toluene/Triton X-100/water emulsions, it 
would be interesting to quantify the degree of packing at the interface. For example, the area occupied by each Triton X-100 molecule upon increasing toluene concentration could be determined using small angle scattering techniques $[130,146]$. Alternatively, the measurement of the total droplet surface area by DLS coupled to the concentration of surfactant molecules at the oil/water interface using filtration techniques $[130,131]$ can also lead to the determination of the surfactant packing.

Additionally the enhancement of the surfactant packing upon increasing oil concentration in toluene-in-water emulsions could be monitored by NMR spin relaxation experiments in the same way to those performed by Choudhury and Schönhoff to probe the dynamics of phenol exchange in dispersions of hollow polyelectrolyte capsules between a free site and a capsule-bound site [147]. For toluene/Triton X-100/water emulsions, the spin-lattice $\left(T_{1}\right)$ and spin-spin $\left(T_{2}\right)$ relaxation constants could be measured using an inversion recovery and a CPMG experiment, respectively, upon varying oil content. The echo decay of the surfactant $T_{1}$ and $T_{2}$ constants is expected to be single exponential as a result of the fast exchange of the surfactant between the droplet surface and the continuous phase, leading to the average of the contributions of the droplet-bound and free sites being detected. The decrease of the surfactant mobility upon increasing packing at the surface of the oil droplets should result in significantly shorter $\mathrm{T}_{1}$ and $\mathrm{T}_{2}$ measured values.

Furthermore two-dimensional diffusion exchange spectroscopy experiments (DEXSY) may provide additional evidence for the increase of surfactant packing in toluene/Triton X-100/water emulsions upon increasing oil content [148]. In these experiments surfactant diffusion is measured in two dimensions after waiting a variable time (called "mixing time") between data points. If exchange of surfactant molecules between the interface and the bulk occurs over the experimental mixing time, off diagonal features may be observed in the twodimensional diffusion spectrum as a result of the change of environment of the surfactant spins. The diffusion coefficients associated with this anisotropy as well as the time scale of the exchange between the interface and the bulk may change upon increasing surfactant packing at the interface. This would be indicative of the enhancement of surfactant packing in toluene/Triton X-100/water emulsions upon increasing oil concentration. However note that both spin relaxation and 
DEXSY experiments could not be performed using oil/Triton X-100/water emulsions where oil is toluene, $p$-xylene or octane due to the low intensity of the Triton X-100 signal as well as the superposition of the surfactant and oil peaks in the NMR spectra.

Finally, the occurrence of oil exchange via an oil permeation or a reversible coalescence mechanism is evidenced for oil/Triton X-100/water emulsions where the oil is one of toluene, $p$-xylene or octane. Despite the significant differences in the properties of these three oil systems in terms of microstructures and destabilization mechanisms, they all undergo oil exchange, indicative of the process universality with respect to oil type. To push the investigation further, it is required to probe the universality of the oil exchange with regard to surfactant type as well. 


\section{REFERENCES}

[1] T. F. Tadros, Applied Surfactants - Principles and Applications, Wiley.

[2] P. Becher, Encyclopedia of Emulsion Technology, Dekker, New York, 1988.

[3] P. Becher, Emulsions: Theory and Practice, Oxford, New York, 2001.

[4] A. W. Schwartz, J. W. Perry, Surface Active Agents, Interscience, New York, 1949.

[5] W. C. Griffin, J. Soc.Cosmetic Chemists 1949, 1, 311.

[6] J. T. Davies, Proceedings, in International Congress on Surface Activity, Vol. 1, London, 1957.

[7] W. C. Griffin, J. Soc.Cosmetic Chemists 1954, 5, 249.

[8] H. Mollet, A. Grubenmann, Formulation Technology, Wiley.

[9] E. J. W. Verwey, J. T. G. Overbeek, Theory of the Stability of Lyophobic Colloids, Elsevier, Amsterdam, 1948.

[10] B. V. Derjaguin, L. Landau, Acta Phsiochim. 1941, 14, 733.

[11] B. V. Derjaguin, L. Landau, J. Exptl. Theoret. Phys. 1941, 11, 802.

[12] H. C. Hamaker, Physica 1937, 4, 1058.

[13] A. E. Alexander, P. Johnson, Colloid Science, University Press, Oxford, 1949.

[14] E. Dickinson, An Introduction to Food Colloids, Oxford, New York, 1992.

[15] H. Helmholtz, Wied. Ann. 1879, 7, 537.

[16] G. C. R. Gouy, J. Phys. Radium 1910, 9, 457.

[17] D. Chapman, Phil. Mag. 1913, 25, 475.

[18] O. Z. Stern, Elektrochem. 1924, 30, 508.

[19] J. N. Israelachvili, Intermolecular and Surface Forces, Academic Press, New York, 1992.

[20] D. Napper, Polymeric Stabilization of Colloidal Dispersion, Academic Press, New York, 1983.

[21] F. T. Hesselink, J. Phys. Chem. 1971, 75, 65.

[22] F. T. Hesselink, A. Vrij, J. T. G. Overbeek, J. Phys. Chem. 1971, 75, 2094.

[23] G. Peschel, P. Belouschek, M. M. Muller, M. R. Muller, R. Konig, Colloid Polym. Sci. 1982, 260(4), 444-451.

[24] Y. I. Rabinovich, B. V. Derjaguin, Colloids and Surfaces 1988, 30(3-4), 243-251.

[25] J. Israelachvili, R. Pashley, Nature 1982, 300(5890), 341-342.

[26] K. Shinoda, H. Arai, J. Phys. Chem. 1954, 68, 3485.

[27] G. H. A. Clowes, J. Phys. Chem. 1916, 20, 407.

[28] P. Sherman, Research 1955, 8, 396.

[29] J. T. Davies, Intern. Congr. Surface Activity, Vol. 2, 1960, p. 585.

[30] W. Rybczynski, Bull. Acad. Sci. 1911, 40.

[31] Hadamard, J. Compt. Rend. 1911, 154, 1735.

[32] A. S. Kabalnov, K. N. Makarov, Kolloidn Zh. 1990, 52, 682.

[33] A. S. Kabalnov, K. N. Makarov, A. V. Pertzov, E. D. Shchukin, J. Colloid Interface Sci. 1990, 138, 98.

[34] A. S. Kabalnov, A. V. Pertzov, E. D. Shchukin, J. Colloid Interface Sci. 1987, 118, 590.

[35] W. I. Higuchi, J. Misra, J. Pharm. Sci. 1962, 51, 459. 
[36] S. Akasura, F. Oosawa, J. Chem. Phys. 1954, 22, 1255.

[37] M. Van Den Tempel, Stability of Oil-in-Water Emulsions, (Ed.: RubberStichting), 1953, p. 34.

[38] M. Van Den Tempel, Rec. Trav. Chim. 1953, 72, 433.

[39] E. Slinde, T. Flatmark, Biochimica Et Biophysica Acta 1976, 455(3), 796-

805.

[40] E. H. Liu, P. T. Callaghan, K. M. McGrath, Langmuir 2003, 19(18), 72497258.

[41] E. H. Liu, K. M. McGrath, Colloid Surf. A-Physicochem. Eng. Asp. 2005, 262(1-3), 101-112.

[42] D. W. Hahn, Light Scattering theory, Department of Mechanical and Aeorospace Engineering, University of Florida, 2006.

[43] I. D. Morrison, S. Ross, Colloidal Dispersions, Wiley-Interscience, New York, 2002.

[44] H. C. van de Hulst, Light Scattering by small particles, Dover.

[45] A. J. Cox, A. J. DeWeerd, J. Linden, Am. J. Phys. 2002, 70(6), 620-625.

[46] B. J. Berne, R. Pecora, Dynamic Light Scattering: with applications to Chemistry, Biology and Physics, Dover.

[47] Size theory, Malvern Instruments, 2008.

[48] B. Balinov, P. Linse, O. Soderman, J. Colloid Interface Sci. 1996, 182(2), 539-548.

[49] H. Egger, K. M. McGrath, J. Colloid Interface Sci. 2006, 299(2), 890-899.

[50] K. J. Packer, C. Rees, J. Colloid Interface Sci. 1972, 40(2), 206-\&.

[51] P. T. Callaghan, K. W. Jolley, R. S. Humphrey, J. Colloid Interface Sci. 1983, 93(2), 521-529.

[52] I. Lonnqvist, A. Khan, O. Soderman, J. Colloid Interface Sci. 1991, 144(2), 401-411.

[53] E. O. Stejskal, J. E. Tanner, J. Chem. Phys. 1965, 42(1), 288-\&.

[54] E. L. Hahn, Physical Review 1950, 80(4), 580-594.

[55] J. E. Tanner, J. Chem. Phys. 1970, 52(5), 2523-\&.

[56] P. T. Callaghan, Aust. J. Phys. 1984, 37(4), 359-387.

[57] P. T. Callaghan, Principles of Nuclear Magnetic Resonance Microscopy, Oxford Univ. Press, New York, 1991.

[58] J. Keeler, Understanding NMR spectroscopy, 2002.

[59] S. Meiboom, D. Gill, Rev. Sci. Instrum. 1958, 29(8), 688-691.

[60] R. Mills, Journal of Physical Chemistry 1973, 77(5), 685-688.

[61] S. W. Provencher, Comput. Phys. Commun. 1982, 27(3), 229-242.

[62] P. T. Callaghan, S. Godefroy, B. N. Ryland, Use of the second dimension in PGSE NMR studies of porous media, in 6th International Conference on the Recent Advances in Magnetic Resonance Applications to Porous Media, Elsevier Science Inc, Ulm, Germany, 2002, pp. 243-248.

[63] M. Minsky, Scanning 1988, 10(4), 128-138.

[64] D. Semwogerere, E. R. Weeks, Encyclopedia of Biomaterials and Biomedical Engineering, Dekker, 2004.

[65]

[66] M. Scivetti, A. Lucchese, V. Crincoli, G. P. Pilolli, G. Favia, Ultrastruct. Pathol. 2009, 33(2), 48-51.

[67] A. Zhivov, O. Stachs, J. Stave, R. F. Guthoff, Br. J. Ophthalmol. 2009, 93(5), 667-672. 
[68] A. D. Dinsmore, E. R. Weeks, V. Prasad, A. C. Levitt, D. A. Weitz, Appl. Optics 2001, 40(24), 4152-4159.

[69] P. J. Lu, J. C. Conrad, H. M. Wyss, A. B. Schofield, D. A. Weitz, Phys. Rev. Lett. 2006, 96(2), 4.

[70] C. P. Royall, A. A. Louis, H. Tanaka, J. Chem. Phys. 2007, 127(4), 8.

[71] T. Moschakis, B. S. Murray, E. Dickinson, Langmuir 2006, 22(10), 47104719 .

[72] C. Bengoechea, F. Cordobes, A. Guerrero, Rheology and microstructure of gluten and soya-based o/w emulsions, in 2nd Annual European Rheology Conference (AERC 2005), Springer, Grenoble, France, 2005, pp. 13-21.

[73] J. J. Bozzola, Electron Microscopy, Jones \& Bartlett, 1998.

[74] J. Goldstein, D. Newbury, Scanning electron microscopy and x-ray microanalysis, Springer, 2003.

[75] Zeta potential theory, Malvern Instruments, 2008.

[76] D. J. McClements, S. R. Dungan, J. B. German, J. E. Kinsella, Food Hydrocolloids 1992, 6(5), 415-422.

[77] D. J. McClements, S. R. Dungan, Colloid Surf. A-Physicochem. Eng. Asp. 1995, 104(2-3), 127-135.

[78] A. Kabalnov, H. Wennerstrom, Langmuir 1996, 12(2), 276-292.

[79] K. Pays, J. Giermanska-Kahn, B. Pouligny, J. Bibette, F. Leal-Calderon, Phys. Rev. Lett. 2001, 87(17), 4.

[80] P. D. I. Fletcher, A. M. Howe, B. H. Robinson, Journal of the Chemical Society-Faraday Transactions I 1987, 83, 985-1006.

[81] S. R. Poulson, R. R. Harrington, J. I. Drever, Talanta 1999, 48(3), 633641.

[82] C. Malmborg, D. Topgaard, O. Soderman, J. Colloid Interface Sci. 2003, 263(1), 270-276.

[83] D. Topgaard, C. Malmborg, O. Soderman, J. Magn. Reson. 2002, 156(2), 195-201.

[84] J. Weiss, J. N. Coupland, D. Brathwaite, D. J. McClements, Colloid Surf. A-Physicochem. Eng. Asp. 1997, 121(1), 53-60.

[85] A. J. I. Ward, Proceedings of the Royal Irish Academy Section BBiological Geological and Chemical Science 1989, 89(6-52), 375-382.

[86] D. J. McClements, S. R. Dungan, Journal of Physical Chemistry 1993, 97(28), 7304-7308.

[87] J. Weiss, J. N. Coupland, D. J. McClements, Journal of Physical Chemistry 1996, 100(3), 1066-1071.

[88] K. Pays, C. Mabille, V. Schmitt, F. Leal-Calderon, J. Bibette, J. Dispersion Sci. Technol. 2002, 23(1-3), 175-186.

[89] L. Taisne, B. Cabane, Langmuir 1998, 14(17), 4744-4752.

[90] V. Schmitt, C. Cattelet, F. Leal-Calderon, Langmuir 2004, 20(1), 46-52.

[91] S. Clark, P. D. I. Fletcher, X. L. Ye, Langmuir 1990, 6(7), 1301-1309.

[92] A. Jada, J. Lang, R. Zana, R. Makhloufi, E. Hirsch, S. J. Candau, Journal of Physical Chemistry 1990, 94(1), 387-395.

[93] Y. Rharbi, M. Li, M. A. Winnik, K. G. Hahn, J. Am. Chem. Soc. 2000, 122(26), 6242-6251.

[94] P. D. I. Fletcher, D. I. Horsup, J. Chem. Soc.-Faraday Trans. 1992, 88(6), 855-864.

[95] M. A. Burschka, C. R. Doering, D. Benavraham, Phys. Rev. Lett. 1989, 63(7), 700-703. 
[96] E.-H. Liu, H. Egger, K. M. McGrath, Journal of Physical Chemistry B 2005, 109(27), 13332-13341.

[97] Y. Q. Song, L. Venkataramanan, L. Burcaw, J. Chem. Phys. 2005, 122(10), 8.

[98] Y. Q. Song, Magn. Reson. Imaging 2007, 25(4), 445-448.

[99] P. D. I. Fletcher, R. Johannsson, J. Chem. Soc.-Faraday Trans. 1994, 90(23), 3567-3572.

[100] S. S. Atik, J. K. Thomas, J. Am. Chem. Soc. 1981, 103(12), 3543-3550.

[101] M. Almgren, J. E. Lofroth, J. Vanstam, Journal of Physical Chemistry 1986, 90(18), 4431-4437.

[102] J. Prost, F. Rondelez, Nature 1991, 350(6319), 11-23.

[103] H. F. Eicke, J. C. W. Shepherd, A. Steinemann, J. Colloid Interface Sci. 1976, 56(1), 168-176.

[104] J. Israelachvili, Colloid Surf. A-Physicochem. Eng. Asp. 1994, 91, 1-8.

[105] L. L. Latour, P. P. Mitra, R. L. Kleinberg, C. H. Sotak, J. Magn. Reson. Ser. A 1993, 101(3), 342-346.

[106] P. P. Mitra, P. N. Sen, L. M. Schwartz, P. Ledoussal, Phys. Rev. Lett. 1992, 68(24), 3555-3558.

[107] P. P. Mitra, P. N. Sen, L. M. Schwartz, Phys. Rev. B 1993, 47(14), 85658574.

[108] D. L. Johnson, T. J. Plona, C. Scala, F. Pasierb, H. Kojima, Phys. Rev. Lett. 1982, 49(25), 1840-1844.

[109] J. W. Haus, K. W. Kehr, Phys. Rep.-Rev. Sec. Phys. Lett. 1987, 150(5-6), 263-406.

[110] L. L. Latour, R. L. Kleinberg, P. P. Mitra, C. H. Sotak, J. Magn. Reson. Ser. A 1995, 112(1), 83-91.

[111] R. W. Mair, G. P. Wong, D. Hoffmann, M. D. Hurlimann, S. Patz, L. M. Schwartz, R. L. Walsworth, Phys. Rev. Lett. 1999, 83(16), 3324-3327.

[112] T. F. Tadros, J. Dispersion Sci. Technol. 1980, 1(4), 465-486.

[113] I. Capek, Adv. Colloid Interface Sci. 2004, 107(2-3), 125-155.

[114] P. Izquierdo, J. Esquena, T. F. Tadros, C. Dederen, M. J. Garcia, N. Azemar, C. Solans, Langmuir 2002, 18(1), 26-30.

[115] B. Deminiere, Modern Aspects of Emulsion Science, The Royal Society of Chemistry, Cambridge, 1998.

[116] C. Wagner, Zeitschrift Fur Elektrochemie 1961, 65(7-8), 581-591.

[117] I. M. Lifshitz, V. V. Slyozov, J. Phys. Chem. Solids 1961, 19(1-2), 35-50.

[118] J. Weiss, C. Canceliere, D. J. McClements, Langmuir 2000, 16(17), 68336838.

[119] R. Chanamai, G. Horn, D. J. McClements, J. Colloid Interface Sci. 2002, 247(1), 167-176.

[120] D. Georgieva, V. Schmitt, F. Leal-Calderon, D. Langevin, Langmuir 2009, 25(10), 5565-5573.

[121] F. Leal-Calderon, Schmitt, V., Bibette, J., Emulsion Science: Basic Principles, Springer, New York, 2007.

[122] K. Shinoda, H. Saito, J. Colloid Interface Sci. 1968, 26(1), 70-\&.

[123] M. Kahlweit, R. Strey, Journal of Physical Chemistry 1987, 91(6), 15531557.

[124] M. Bourrel, J. L. Salager, R. S. Schechter, W. H. Wade, J. Colloid Interface Sci. 1980, 75(2), 451-461. 
[125] M. E. Hayes, M. Bourrel, M. M. Elemary, R. S. Schechter, W. H. Wade, Society of Petroleum Engineers Journal 1979, 19(6), 349-356.

[126] H. T. Davis, Colloid Surf. A-Physicochem. Eng. Asp. 1994, 91, 9-24.

[127] J. N. Israelachvili, D. J. Mitchell, B. W. Ninham, Journal of the Chemical Society-Faraday Transactions Ii 1976, 72, 1525-1568.

[128] V. T. Liveri, Controlled synthesis of nanoparticles in microheterogeneous systems, 2006.

[129] R. Nagarajan, Langmuir 2002, 18(1), 31-38.

[130] K. Oehlke, V. A. Garamus, A. Heins, H. Stockmann, K. Schwarz, J. Colloid Interface Sci. 2008, 322(1), 294-303.

[131] M. A. James-Smith, K. Alford, D. O. Shah, J. Colloid Interface Sci. 2007, 315(1), 307-312.

[132] A. Kabalnov, T. Tarara, R. Arlauskas, J. Weers, J. Colloid Interface Sci. 1996, 184(1), 227-235.

[133] E. H. Liu, H. Egger, K. M. McGrath, J. Phys. Chem. B 2005, 109(27), 13332-13341.

[134] V. Schmitt, F. Leal-Calderon, Europhys. Lett. 2004, 67(4), 662-668.

[135] J. Weiss, N. Herrmann, D. J. McClements, Langmuir 1999, 15(20), 6652-

6657.

[136] J. Weiss, D. J. McClements, Langmuir 2000, 16(14), 5879-5883.

[137] S. R. Dungan, B. H. Tai, N. I. Gerhardt, Colloid Surf. A-Physicochem. Eng. Asp. 2003, 216(1-3), 149-166.

[138] A. A. Pena, C. A. Miller, Adv. Colloid Interface Sci. 2006, 123, 241-257.

[139] S. Karaborni, N. M. Vanos, K. Esselink, P. A. J. Hilbers, Langmuir 1993, 9(5), 1175-1178.

[140] K. Esselink, P. A. J. Hilbers, N. M. Vanos, B. Smit, S. Karaborni, Colloid Surf. A-Physicochem. Eng. Asp. 1994, 91, 155-167.

[141] J. Saien, S. Akbari, J. Chem. Eng. Data 2006, 51(5), 1832-1835.

[142] T. Al-Sahhaf, A. Elkamel, A. S. Ahmed, A. R. Khan, Chem. Eng. Commun. 2005, 192(5), 667-684.

[143] M. Kahlweit, R. Strey, Angew. Chem.-Int. Edit. Engl. 1985, 24(8), 654668.

[144] B. Jajuee, A. Margaritis, D. Karamanev, M. A. Bergougnou, Chem. Eng. Sci. 2006, 61(12), 4111-4119.

[145] A. I. Rusanov, V. V. Krotov, Colloid J. 2004, 66(2), 204-207.

[146] H. Kunieda, G. Umizu, K. Aramaki, J. Phys. Chem. B 2000, 104(9), 2005-

2011.

[147] R. P. Choudhury, M. Schonhoff, J. Chem. Phys. 2007, 127(3).

[148] P. L. Hubbard, K. M. McGrath, P. T. Callaghan, Langmuir 2005, 21, 4340-4346. 